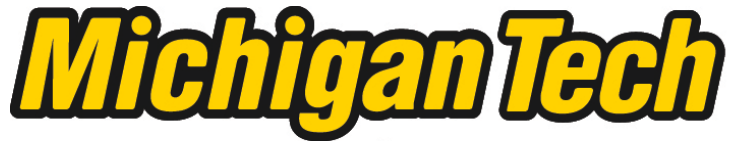 \\ Michigan Technological University Create the Future Digital Commons @ Michigan Tech
}

Dissertations, Master's Theses and Master's Reports - Open

Dissertations, Master's Theses and Master's

Reports

2010

PMMA-CNT matrices for vacuum electronic, biosensing and energy applications

Archana Pandey

Michigan Technological University

Follow this and additional works at: https://digitalcommons.mtu.edu/etds

Part of the Physics Commons

Copyright 2010 Archana Pandey

\section{Recommended Citation}

Pandey, Archana, "PMMA-CNT matrices for vacuum electronic, biosensing and energy applications", Dissertation, Michigan Technological University, 2010.

https://doi.org/10.37099/mtu.dc.etds/115

Follow this and additional works at: https://digitalcommons.mtu.edu/etds

Cart of the Physics Commons 


\title{
PMMA-CNT Matrices for Vacuum Electronic, Biosensing and Energy Applications
}

By

\author{
Archana Pandey
}

\section{A DISSERTATION \\ Submitted in partial fulfillment of the requirements for the degree of DOCTOR OF PHILOSOPHY \\ (Engineering Physics)}

\author{
MICHIGAN TECHNOLOGICAL VNIVERSITY \\ 2010
}

Copyright (C) Archana Pandey 2010 

This dissertation, "PMMA-CNT Matrices for Vacuum Electronic, Biosensing and Energy Applications," is hereby approved in partial fulfillment of the requirements for the degree of Doctor of Philosophy in the field of Engineering Physics.

\author{
Department: Physics
}

Research Advisor and

Chair of Advisory Committee:

Dr. Yoke Khin Yap

Committee Members:

Dr. Craig Friedrich

Dr. Miguel Levy

Dr. Ranjit Pati

Physics Department Chair:

Dr. Ravindra Pandey

Date: 

Dedicated to the loving memory of my Aunt Prabhavati Devi

\author{
$1945-2003$ \\ My family and My Love \\ Abhishek Prasad
}





\section{Contents}

List of Figures $\quad$ xi

List of Tables $\quad$ xiv

Preface $\quad$ XV

Acknowledgments xvii

Units and Abbreviations $\quad$ xxi

Abstract $\quad$ xxiii

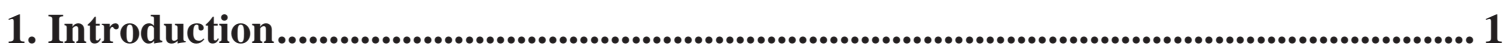

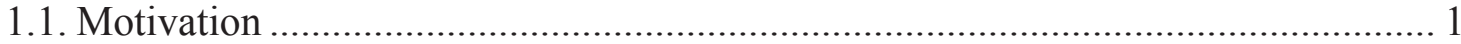

1.2. Background and classification of carbon nanotubes .......................................... 2

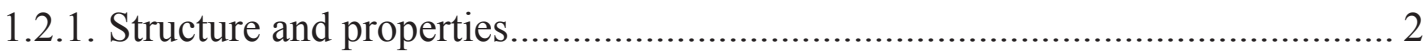

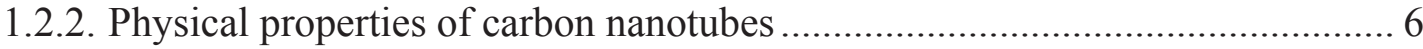

1.3. Synthesis methods of carbon nanotubes.......................................................... 7

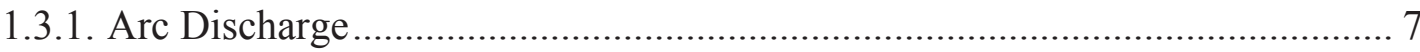

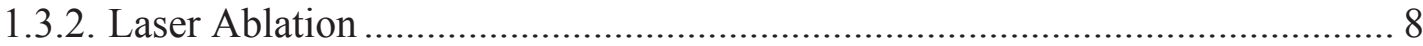

1.3.3. Chemical vapor deposition technique (CVD) ........................................ 10

1.3.4. Plasma enhanced chemical vapor deposition (PECVD) ............................. 11

1.4. Growth modes and optimization of growth parameters ..................................... 13

1.4.1 Effect of flow rate on growth of CNTs...................................................... 13

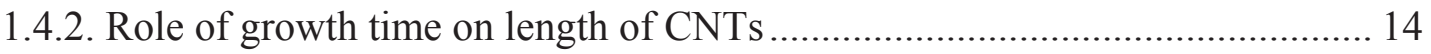

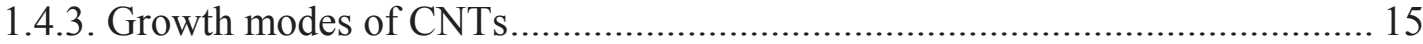

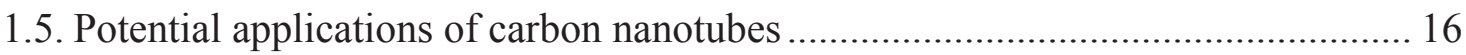

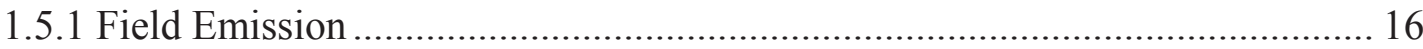

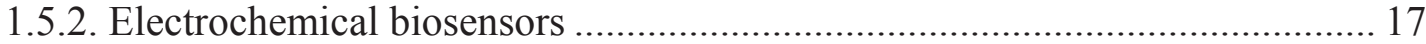

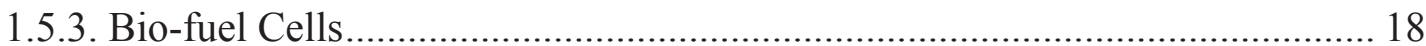

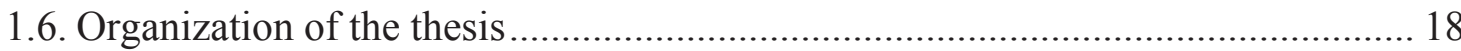

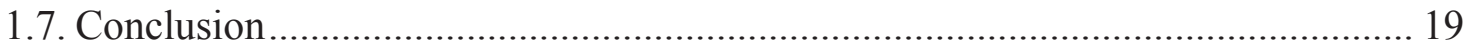

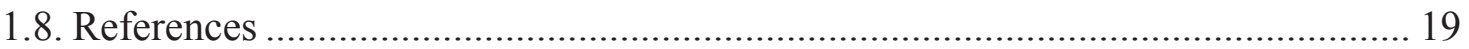


2. Investigation methods............................................................................................ 25

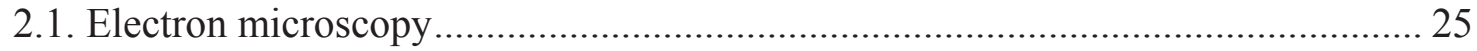

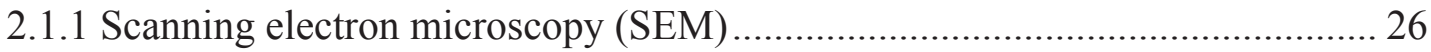

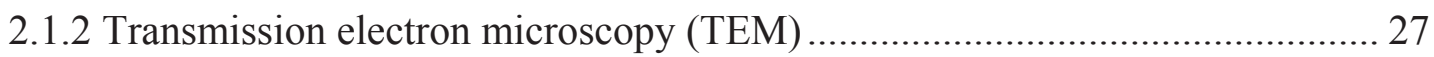

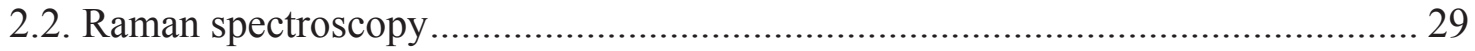

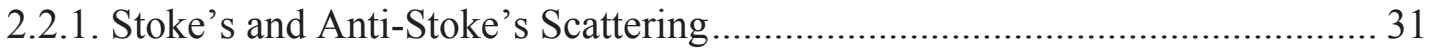

2.2.2. Raman spectroscopy of multi-walled carbon nanotubes (MWCNTs)............. 32

2.3 Fourier Transform Infrared (FTIR) Spectroscopy ………………………........... 35

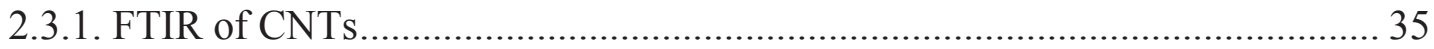

2.4 Cyclic Voltammetry (CV) .................................................................................... 36

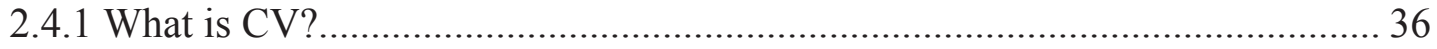

2.4.2 Electrochemical cell and electrodes used in CV experiments.......................... 36

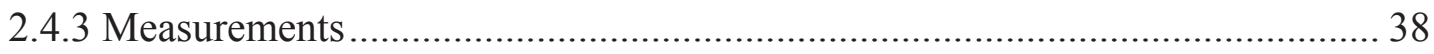

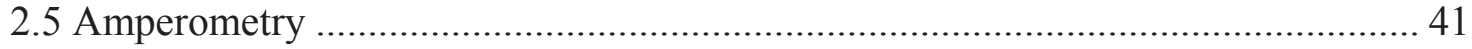

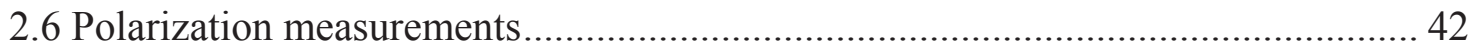

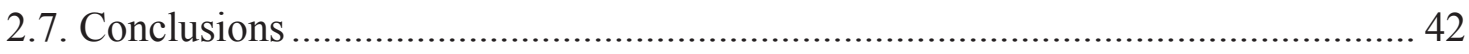

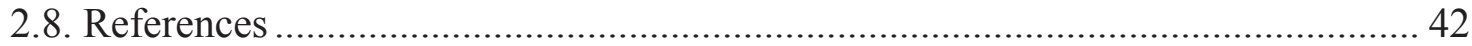

3. Enhanced Field emission from CNTs................................................................................... 45

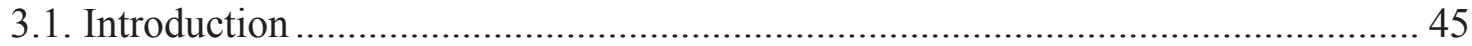

3.1.1. Field emission theory .................................................................................... 45

3.2. Current state of research in field emission from CNTs......................................... 46

3.3. Experimental methods to enhance the field emission properties from as grown

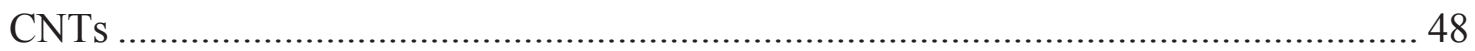

3.3.1. Fabrication of etched and conical self-assembled bundles of CNTs............... 48

3.3.2. Fabrication of PMMA-CNT matrices............................................................... 49

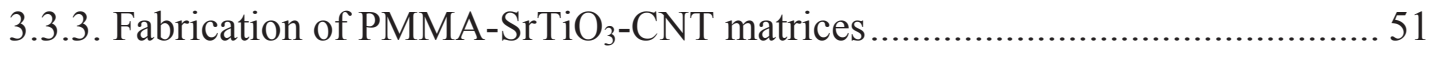

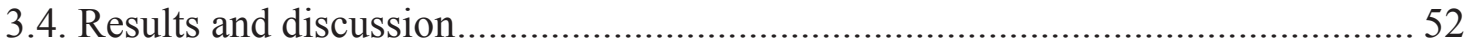

viii 
3.4.1. Field emission from etched and conical self-assembled bundles of CNTs .... 52

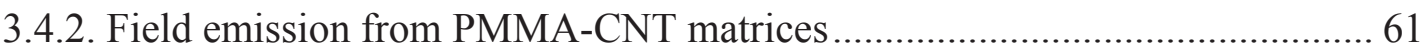

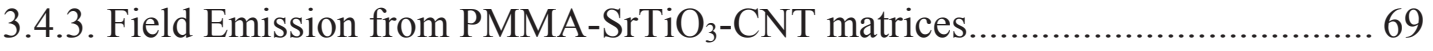

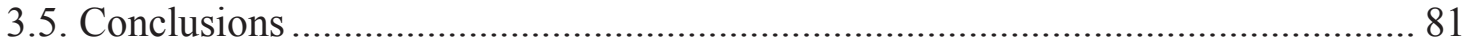

3.6. Future work on Field Emission .................................................................. 83

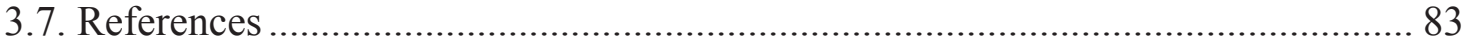

4. Glucose Biosensors Based on PMMA-CNT Matrices.............................................. 87

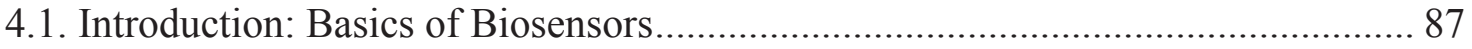

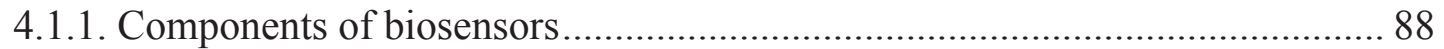

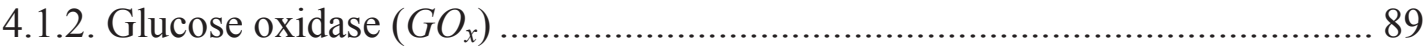

4.2. Current state of research in biosensors based on CNTs .................................... 90

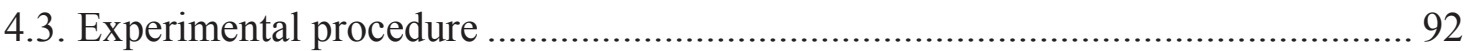

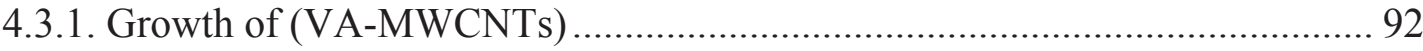

4.3.2. Fabrication of PMMA-CNT matrices......................................................... 93

4.3.3. Preparation of biosensor with different working electrode area..................... 95

4.3.4. Functionalization of exposed tips of PMMA-CNT matrices with - $\mathrm{COOH}$

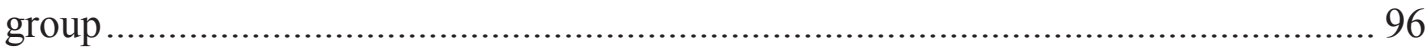

4.3.5. Immobilization of $G O_{x}$ on PMMA-CNT matrices ..................................... 98

4.3.6. Assembling glucose biosensor and its measurement................................... 99

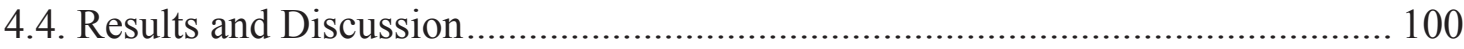

4.4.1. Cyclic Voltammetry (CV) measurements .............................................. 100

4.4.2. Effect of electrode area on performance of biosensors ............................... 105

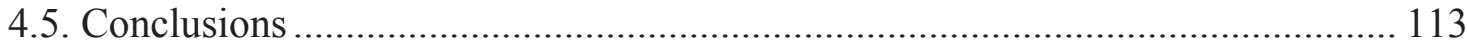

4.6. Future Work on Glucose Biosensors............................................................... 114

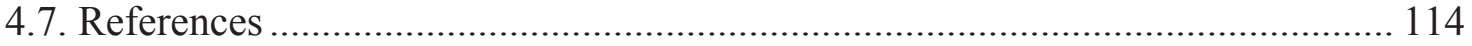


5. Energy Generation and Storage using Functionalized PMMA-CNT Matrices.. 119

5.1. Introduction to the Energy Sensing, Generation and Storage (SGS) System ...... 119

5.2. Biofuel cells: A Brief Look.............................................................................. 121

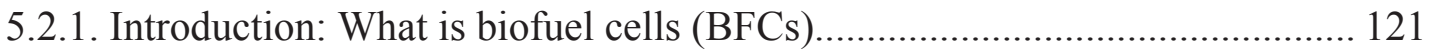

5.2.2. Enzyme based BFCs: Related Issues..................................................... 124

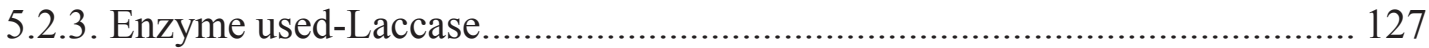

5.3. Key performance characteristics and terms for BFCs.................................. 128

5.4. Experimental details-Assembling Membrane-less BFCs................................. 131

5.4.1. Fabrication of PMMA-CNT bioanode for BFCs...................................... 131

5.4.2. Fabrication of PMMA-CNT biocathode for BFCs................................... 132

5.4.3. Biofuel cell fabrication and characterization............................................ 132

5.4.4. Fabrication of BFCs with different area .................................................... 133

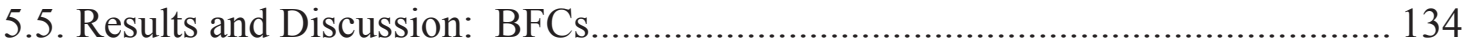

5.5.1. CV of laccase functionalized PMMA-CNT matrices as biocathode............. 134

5.5.2. Polarization measurements of assembled BFCs .......................................... 134

5.5.3. Effect of different electrode area on performance of BFCs ........................ 135

5.6. Supercapacitors as Energy Storage Devices: An Introduction........................... 144

5.7. Experimental: Fabrication of PMMA-CNT matrices based supercapacitor ........ 147

5.8. Results and Discussion: Performance of PMMA-CNT Supercapacitors............. 149

5.9. Conclusions ……………………………………........................................... 153

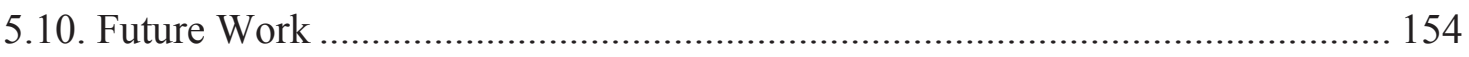

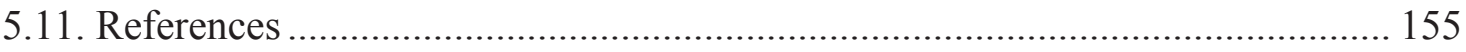

6. Future work on energy SGS system based on PMMA-CNT matrices................. 167

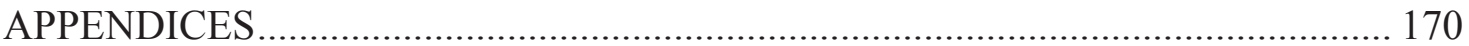

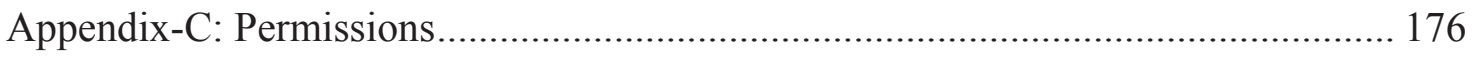




\section{List of Figures}

Figure 1.1: Single walled carbon nanotubes ............................................................. 3

Figure 1.2: Multi-walled carbon nanotubes .............................................................. 3

Figure 1.3: Rolling single walled carbon nanotubes in different ways............................ 4

Figure 1.4: A graphene sheet showing different terms ................................................. 5

Figure 1.5: Illustration of an arc-discharge apparatus. ............................................ 8

Figure 1.6: Illustration of a laser ablation apparatus................................................. 9

Figure 1.7: Picture of a horizontal CVD furnace...................................................... 10

Figure 1.8: Schematic diagram and Actual picture of PECVD system ......................... 12

Figure 1.9: Variation of methane flow rate with the length of CNTs ........................... 13

Figure 1.10: Variation of growth time with the length of CNTs.......................14

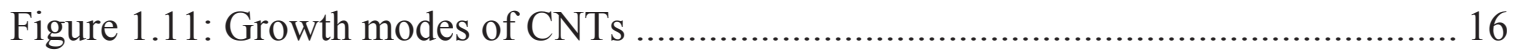

Figure 2.1: High-energy electron beam interaction with a thin specimen..................... 25

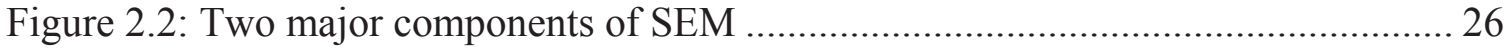

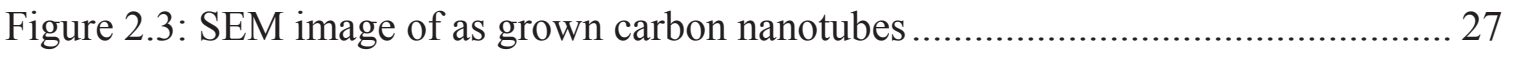

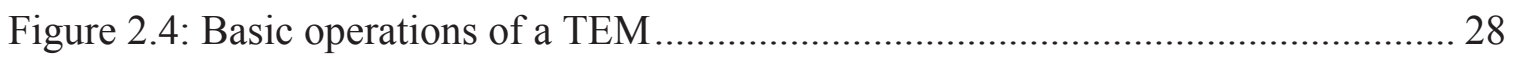

Figure 2.5: TEM image of as grown carbon nanotubes ............................................ 29

Figure 2.6: Different kinds of scattering of light photons ........................................ 31

Figure 2.7: Raman vibration modes in carbon nanotubes.......................................... 32

Figure 2.8: Raman spectrum of multi-walled CNTs.................................................. 34

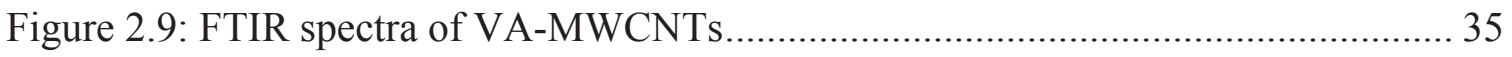

Figure 2.10: Typical cell and electrode setup used for cyclic voltammetry .................... 37

Figure 2.11: Current potential curve for a cyclic voltammetry measurement ................ 39

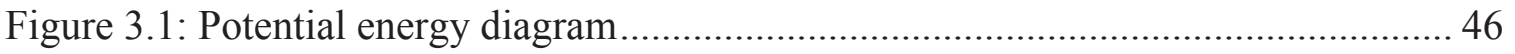

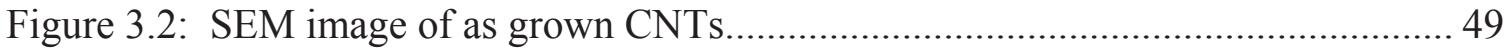


Figure 3.3: Schematic for the fabrication of PMMA-CNT matrices ................................ 50

Figure 3.4: Schematic for the fabrication of PMMA-STO-CNT matrices ....................... 52

Figure 3.5: Typical appearance of opened-tip VA-MWCNTs .......................................... 53

Figure 3.6: Raman spectra of as grown and the etched VA-MWCNTs ........................... 54

Figure 3.7: Field emission curves for the as grown and the etched VA-MWCNTs ......... 56

Figure 3.8: Schematic of the simulated potential maps ................................................... 59

Figure 3.9: SEM images and Raman spectra of PMMA-CNT matrices............................. 62

Figure 3.10: The emission current characteristics of PMMA-CNT matrices ................... 63

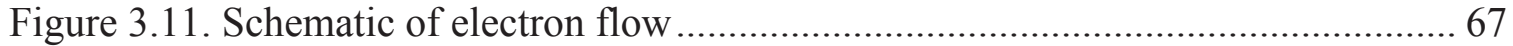

Figure 3.12: SEM images and Raman spectra PMMA-STO-CNTs................................ 70

Figure 3.13: XPS spectra and TEM images of the as-grown and STO MWCNTs. ....... 72

Figure 3.14: Field emission curves and potential maps for PMMA-STO-CNTs............. 74

Figure 3.15: Emission current stability curves. .............................................................. 80

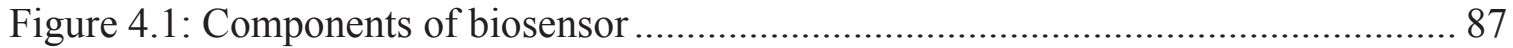

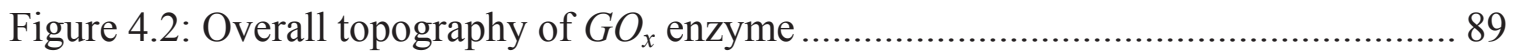

Figure 4.3: Schematic and SEM of as grown VA-MWCNTs ......................................... 93

Figure 4.4: Schematic diagram and SEM image PMMA-CNT matrices ......................... 94

Figure 4.5: The schematic diagram of CNTs with different areas.................................... 95

Figure 4.6: Functionalization of VA-MWCNTs.......................................................... 97

Figure 4.7: Reaction involving EDC and Sulfo-NHS .................................................... 98

Figure 4.8: Electrochemical cell ................................................................................... 99

Figure 4.9: $\mathrm{CV}$ and Amperometric of $G O_{x}$-PMMA-CNTs biosensor. ............................ 101

Figure 4.10: Amperometric response of $G O_{x}$-PMMA-CNT biosensor. .......................... 103

Figure 4.11: CV of $G O_{x}$-PMMA-CNT biosensor.......................................................... 106

Figure 4.12: CV of $G O_{x}$-PMMA-CNT biosensor ......................................................... 107

Figure 4.13: Amperometric response of $G O_{x}$-PMMA-CNT with different areas. ......... 108

Figure 4.14: Calibration curve of $G O_{x}$-PMMA-CNT biosensor..................................... 110 
Figure 5.1: Schematic representation of SGS concept............................................. 119

Figure 5.2: Schematic of Enzymatic Biofuel Cells..................................................... 123

Figure 5.3: Structure and active sites of Laccase from T. versicolor .......................... 127

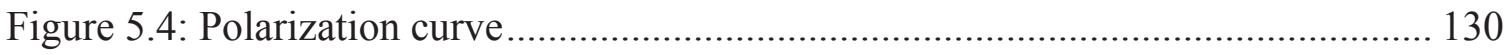

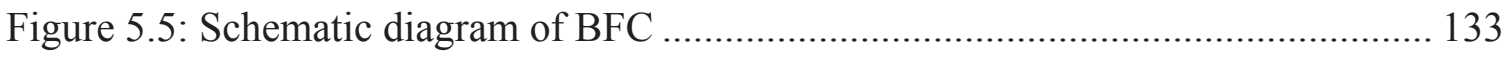

Figure 5.6: CV of Laccase immobilized PMMA-CNT matrices.................................. 134

Figure 5.7: Polarization curve and Power output of $G O_{x}$-PMMA-CNT. ..................... 135

Figure 5.8: CV of Laccase immobilized PMMA-CNT BFCs .................................... 136

Figure 5.9: Polarization and Power output of BFCs.................................................. 137

Figure 5.10: Schematic of fuel flow from different directions .................................. 143

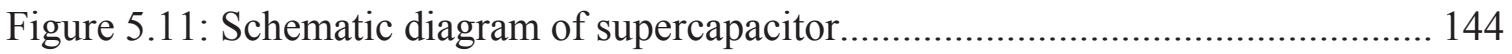

Figure 5.12: Schematic diagram of PMMA-CNTs based supercapacitor ..................... 148

Figure 5.13: Different component of the supercapacitors ........................................ 148

Figure 5.14: CV of the PMMA-CNT matrices based supercapacitor........................... 150

Figure 5.15: Galvanostatic charge-discharge curve of the PMMA-CNT matrices ........ 151

Figure 6.1: Circuit block diagram for SGS ........................................................... 168

Figure 6.2: Circuit diagram for charging and discharging of SGS device.................... 168

Figure 1B-1: Linear fitting of calibration curves ............................................... 171

Figure 1B-2: Linear fitting of calibration curves ..................................................... 172

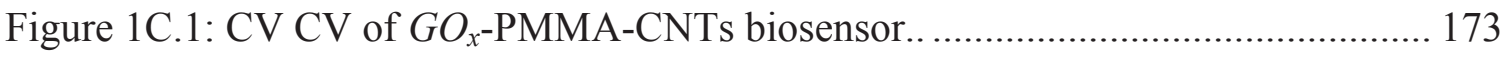

Figure 1C.2: CV of $G O_{x}$-PMMA-CNTs biosensor............................................... 174

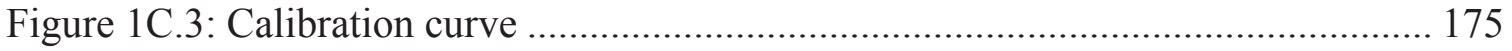




\section{List of Tables}

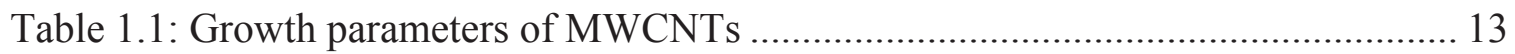

Table 4.1: Variation of biosensor parameters with different electrode areas ................ 111

Table 5.1: Variation in parameters of BFCs with different electrode areas .................. 138 


\section{PREFACE}

Chapter 1 (Introduction) presents various growth techniques of carbon nanotubes (CNTs) which includes arc discharge, laser ablation and chemical vapor deposition (CVD) techniques. Growth of CNTs by our dual radio frequency plasma enhanced CVD is described in detail. Finally the optimization of various growth parameters is discussed.

Chapter 2 (Investigation methods) discusses various experimental techniques for the characterization of CNTs and the device fabrication, including scanning electron microscopy (SEM), transmission electron microscopy (TEM), Raman spectroscopy, Fourier transform infra-red spectroscopy, and electrochemical techniques.

Chapter 3 (Enhanced Field emission from CNTs) provides the basics of electron field emission. Thereafter, the issues involved in field emission of as grown CNTs are discussed. This is followed by the experimental details and discussion of our approaches in overcoming some of these issues.

Chapter 4 (Glucose biosensors based on PMMA-CNT Matrices) introduces glucose biosensors based on our PMMA-CNT matrices. The biosensor was developed using immobilization of glucose oxidase $\left(G O_{x}\right)$ on CNTs. The durability, stability and sensitivity of the biosensor were also studied. In order to understand and improve the performance of miniaturized glucose biosensors we have then investigated the effect of working electrode area on the sensitivity and current level of our biosensors.

Chapter 5 (Energy Generation and Storage using Functionalized PMMA-CNT Matrices) introduces the concept of energy sensing, generation and storage (SGS) devices using 
functionalized PMMA-CNT matrices. At the beginning, this chapter introduces the concept of biofuel cells (BFCs) for energy generation using PMMA-CNT matrices. The construction of these BFCs and the effect of the electrode area on the power density of these BFCs are then described. Then, we have investigated the use of PMMA-CNT matrices as supercapacitors for energy storage devices. The performance of these supercapacitors and ways to enhance their performance are discussed.

Chapter 6 (Future work on SGS based on PMMA-CNT matrices) discusses the future work of the proposed SGS devices. 


\section{Acknowledgments}

This work was not at all possible without the support of numerous people. It is hard to name them all but I'll try my best to acknowledge them all.

I am very grateful to my adviser, Dr Yoke Khin Yap, whose encouragement, guidance and support from the initial through the final stages enabled me to develop an understanding of the subject. Most importantly I am grateful for the trust he had in me. I would also like to thank my committee members, Dr Craig Friedrich, Dr Ranjit Pati, and Dr Miguel Levy for their time, comments and guidance throughout this work. It has been my privilege to work with them.

I owe my deepest gratitude to the Department of Physics at Michigan Technological University for their constant support. I will always be indebted to Dr Ravindra Pandey for giving me the opportunity to join the department and do this work. If it was not for him I would have never come so far. Thank you very much for your patience and for believing in me. I would also like to thank Mrs Aparna Pandey for acting as a guardian when we needed her most.

I am very thankful to our department secretaries Kathy Wollan, Marg Rohrer, Elizabeth Pollins and Andrea Lappi for the support they gave me through all the years. Thank you, Kathy, for your friendship and offering us your cabin. We really had good times there.

In my daily work I have been blessed with a friendly and cheerful group of fellows. My progress through the program was made easier every step of the way with the support of

xvii 
my fellow researchers Vijaya Kayastha, Jiesheng Wang, Benjamin Ulmen, Samuel Mensah, Jason Moscatello, Ming Xie, Michael Gussert, Chee Huei Lee, Abhay Pratap Singh, Sanjay Karna, Shun Wu, Ravi Chintala, Vamsi Kunapuli and Boyi Hao. Mike Gussert and Ben Ulmen deserve special thanks as I learned a lot from them. Thank you, Ben and Mike, for all the help you have given me during my stay at MTU. I would like to show my gratitude to Dr Donald Beck, Dr Brian Fick, and Dr Petra Huentemeyer for their countless and helpful discussions.

I would also like to thank Dr Shashi Karna for his initial comments on my field emission work. A special thanks to Dr Dawdon Cheam for teaching us cleanroom techniques. Thank you for all your time and support. Very special thanks go to Mr. Owen Mills for training us and for being there for us for all the equipment training and help. It is because of him that we had hands-on experience in many characterization techniques. I would also like to thank Mr. Bill Knudsen for his training in cleanroom equipments.

My sincere thanks go to Mr. Wil Slough for all that he's done for me. Thank you, Wil, for all your help, your advice, and all your stories. I cannot go without saying thank you to Mr. Jesse Nordeng for helping me throughout my entire $\mathrm{PhD}$ in repairing the heater without which this work would not have been possible.

Along this journey I made many friends at MTU. I would also like to convey my thanks to all my friends at MTU who made this possible. My thanks also go to Madhusudan Savaikar, Pushpamitra Panigrahi, Himanshu Verma, Asha Lakkavaram, Kevin Cassell, Jodhbir Singh, Suryabh Sharma, and Clara Anton Fernandez. I was extraordinarily xviii 
fortunate to have a friend like Clara. Thank you for your friendship, your support as a best friend, and most of all thank you for listening to me.

I would also like to thank Debra Charlesworth for all the effort she put into my thesis work. It is really amazing how much work she does. It is astonishing that she reads and provides helpful feedback on all the theses written by Michigan Tech graduate students. Thank you, Debra, for all your effort in making our thesis a memorable document. I would also like to thank Nancy Sprague for all her help and support she provided me during my stay at MTU.

Where would I be without my family? I owe my deepest gratitude to my parents and my grandfather for letting me come this far and supporting me in every step of my life. To my best friend and sister I owe everything and my success is her success. Thank you, Aradhana, for being there for me when I needed you most. Thank you for listening to me when I had no one to go to and thank you for those long hours of talk. Thank you for your enthusiasm, your support, and best of all your friendship. I am here for a reason and that reason is my brother. I would like to show my gratitude to my brother, Shishir Pandey who has helped me grow as a person. Thank you for all the humorous stories you told me and for all the wonderful things you have done for me.

Words fail me when I try to express my appreciation to my everlasting love Abhishek Prasad. Your dedication, love and persistent confidence in me have taken the load off my shoulder. I would have never made it so far without your unconditional love, support, 
patience and friendship. Thank you for making my life meaningful and, most of all thank you for walking beside through the rest of our life together.

Last but not least, I would like to thanks my mentors Dr Theva Thevuthasan, Dr. Mark Engelhard, Dr Ponnusamy Nachimuthu and Dr Chongmin Wang at Pacific Northwest National Laboratory (PNNL) for giving me opportunity to work in their lab as an intern. I learned a lot from them and it was very nice experience.

Finally, I would like to thank everybody who was important to the successful completion of my thesis, and I regret that I don't have the space to elaborate the importance each person has played in this endeavor. 


\section{Units and Abbreviations}

\begin{tabular}{|c|c|}
\hline CNTs & Carbon Nanotube \\
\hline CVD & Chemical Vapor Deposition \\
\hline SWCNT & Single-Walled Carbon Nanotube \\
\hline MWCNT & Multi-Walled Carbon Nanotubes \\
\hline PECVD & Plasma-Enhanced Chemical Vapor Deposition \\
\hline PLD & Pulsed Laser Deposition \\
\hline $\mathrm{CV}$ & Cyclic Voltammetry \\
\hline $\mathrm{AgCl}$ & Silver Chloride \\
\hline FESEM & Field Emission Scanning Electron Microscope \\
\hline AFM & Atomic Force Microscope \\
\hline FTIR & Fourier Transform Infrared Spectrometer \\
\hline G/D & Graphitic Peak Intensity to Disorder Peak Intensity Ratio \\
\hline RBM & Radial Breathing Mode \\
\hline SCCM & standard cubic centimeter per minute \\
\hline SEM & Scanning Electron Microscope \\
\hline TEM & Transmission Electron Microscope \\
\hline
\end{tabular}


xxii 


\section{Abstract}

Carbon nanotubes (CNTs) are interesting materials with extraordinary properties for various applications. Here, vertically-aligned multiwalled CNTs (VA-MWCNTs) are grown by our dual radio frequency plasma enhanced chemical vapor deposition (PECVD). After optimizing the synthesis processes, these VA-MWCNTs were fabricated in to a series of devices for applications in vacuum electronics, glucose biosensors, glucose biofuel cells, and supercapacitors In particular, we have created the so-called PMMA-CNT matrices (opened-tip CNTs embedded in poly-methyl methacrylate) that are promising components in a novel energy sensing, generation and storage (SGS) system that integrate glucose biosensors, biofuel cells, and supercapacitors. The content of this thesis work is described as follows:

1. We have first optimized the synthesis of VA-MWCNTs by our PECVD technique. The effects of $\mathrm{CH}_{4}$ flow rate and growth duration on the lengths of these CNTs were studied.

2. We have characterized these VA-MWCNTs for electron field emission. We noticed that as grown CNTs suffers from high emission threshold, poor emission density and poor long-term stability. We attempted a series of experiments to understand ways to overcome these problems. First, we decrease the screening effects on VA-MWCNTs by creating arrays of self-assembled CNT bundles that are catalyst-free and opened tips. These bundles are found to enhance the field emission stability and emission density. Subsequently, we have created PMMA-CNT matrices that are excellent electron field emitters with an emission threshold field of more than two-fold lower than that of the as-grown sample. Furthermore, no significant emission degradation was observed after a continuous emission test of 40 hours (versus much shorter tests in reported literatures). Based on the new understanding we learnt from the PMMACNT matrices, we further created PMMA-STO-CNT matrices by embedding openedtip VA-MWCNTs that are coated with strontium titanate $\left(\mathrm{SrTiO}_{3}\right)$ with PMMA. We found that the PMMA-STO-CNT matrices have all the desired properties of the xxiii 
PMMA-CNT matrices. Furthermore, PMMA-STO-CNT matrices offer much lower emission threshold field, about five-fold lower than that of as grown VA-MWCNTs. The new understandings we obtained are important for practical application of VAMWCNTs in field emission devices.

3. Subsequently, we have functionalized PMMA-CNT matrices for glucose biosensing. Our biosensor was developed by immobilized glucose oxidase $\left(G O_{x}\right)$ on the openedtip CNTs exposed on the matrices. The durability, stability and sensitivity of the biosensor were studied. In order to understand the performance of miniaturized glucose biosensors, we have then investigated the effect of working electrode area on the sensitivity and current level of our biosensors.

4. Next, functionalized PMMA-CNT matrices were utilized for energy generation and storage. We found that PMMA-CNT matrices are promising component in glucose $/ \mathrm{O}_{2}$ biofuel cells (BFCs) for energy generation. The construction of these BFCs and the effect of the electrode area on the power density of these BFCs were investigated. Then, we have attempted to use PMMA-CNT matrices as supercapacitors for energy storage devices. The performance of these supercapacitors and ways to enhance their performance are discussed.

5. Finally, we further evaluated the concept of energy SGS system that integrated glucose biosensors, biofuel cells, and supercapacitors. This SGS system may be implantable to monitor and control the blood glucose level in our body. 


\section{Chapter 1}

\section{Introduction}

\subsection{Motivation}

"Nano" a small word but technology associated with it, has changed the world in an incredible way. Now a days nanotechnology has affected and is changing the existing technology in a farfetched way. The research in the domain of CNTs has contributed significantly towards the potential aspects of Nanotechnology. Keeping this stimulus to contribute in this domain, I have decided to study the applications of CNTs. The project was started on the optimization of growth conditions of MWCNTs using our PECVD system. And then I moved on to the potential electronic, biological and energy applications. At first, I focused on using CNTs as field emission devices. These devices are suffering from various issues including high threshold voltage and low stability. To overcome these deficiencies, I used CNTs embedded in PMMA (PMMA-CNT matrices) to enhance the field emission properties. Another application for potential use of CNTs is in glucose biosensors. Functionalized PMMA-CNT matrices act as excellent biosensors with high enzyme lifetimes and long term stability. An exciting application explored is biofuel cell. Functionalized PMMACNT matrices act as excellent biofuel cells with high power density and efficiency. As described, major portion of this work is based on the use of PMMA-CNT matrices in various applications. 


\subsection{Background and classification of carbon nanotubes}

The atomic number of Carbon is six and has lowest atomic number in column four of the periodic table. CNTs are seamless cylinders of graphitic carbon with extraordinary properties. Tremendous increase in interest in CNTs is a consequence of synthesis of buckminsterfullerene $\mathrm{C}_{60}$, and other structures in 1985. Later in another experiment by W. Krätschmer and D. Huffman discovered to produce macroscopic quantities of fullerenes. Since the invention CNTs have attracted great interest in diverse applications because of their excellent properties [1-3].

\subsubsection{Structure and properties}

Carbon-based nanomaterials are distinctive and versatile in various ways. Carbon can form different possible structures by combining two or more covalently bonded atoms, which is known as the hybridization of atomic orbitals. Therefore, three types of hybridizations occur in carbon: $s p, s p^{2}$ and $s p^{3}$. In Carbon, the inner atomic orbitals are absent and (only 1s orbital is present) because of the absence of inner atomic orbitals the hybridizations in carbon involves only valence $s$ and $p$ orbitals [2, 3]. Carbon nanotubes are the most suitable nanoscale materials for nanotechnology applications. 


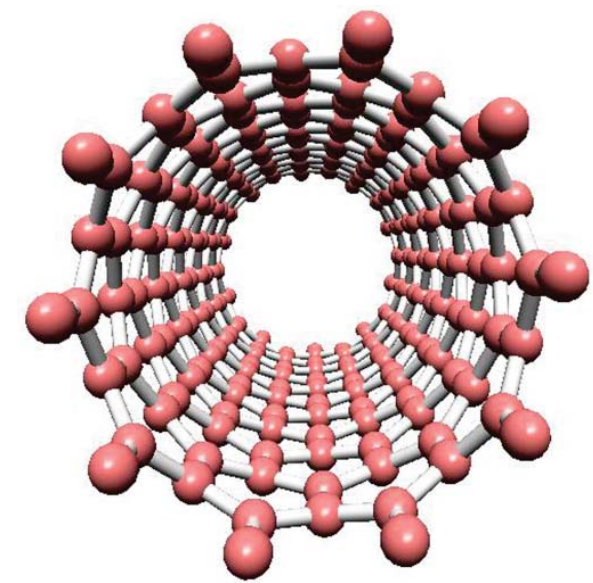

Figure 1.1: Single walled carbon nanotubes

As the name suggests, a single walled carbon nanotubes (SWCNTs) can be described as a rolled up graphene sheet into a form of cylinder. Figure 1.1 shows the end-view of a SWCNT. A multi-walled carbon nanotube (MWCNT) consists of a series of co-axial concentric SWCNTs as shown in Figure 1.2.

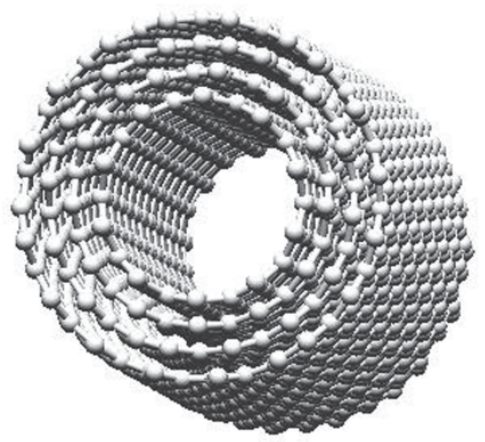

Figure 1.2: Multi-walled carbon nanotubes

A nanotube is usually characterized by its diameter $d$ and chiral angle $\theta(0 \leq|\theta|$ $\leq 30^{\circ}$ ) (Figure 1.3). The chiral vector $C_{h}$ is defined with the two integers $(\mathrm{n}, \mathrm{m})$ and the basis vectors of the graphene sheet [3]. Typical diameter of SWCNTs is of the 
order of $1.5 \mathrm{~nm}$. The manner in which the graphite sheet rolls up is called the "Chirality". The chiral vector is given by the two integers (n, $\mathrm{m}$ ) and the basis vectors of the graphene sheet and is given by: $\quad C_{h}=\mathrm{n} \boldsymbol{a}_{1}+\mathrm{m} \boldsymbol{a}_{2}=(\mathrm{n}, \mathrm{m})$

The size of the nanotubes and their properties are determined by the "Chiral Vector" where, $\boldsymbol{C}_{\boldsymbol{h}}=$ circumference of the CNT, $\mathrm{a}_{1}$ and $\mathrm{a}_{2}$ are the unit vectors and $\mathrm{n}$ and $\mathrm{m}$ are the integers. CNTs can be either metallic or semiconducting depending on their geometry. SWNTs are metallic when $n-m=3 p$, where $p$ is integer. With all other values of $\mathrm{n}$ and $\mathrm{m}$ it will be semiconducting.

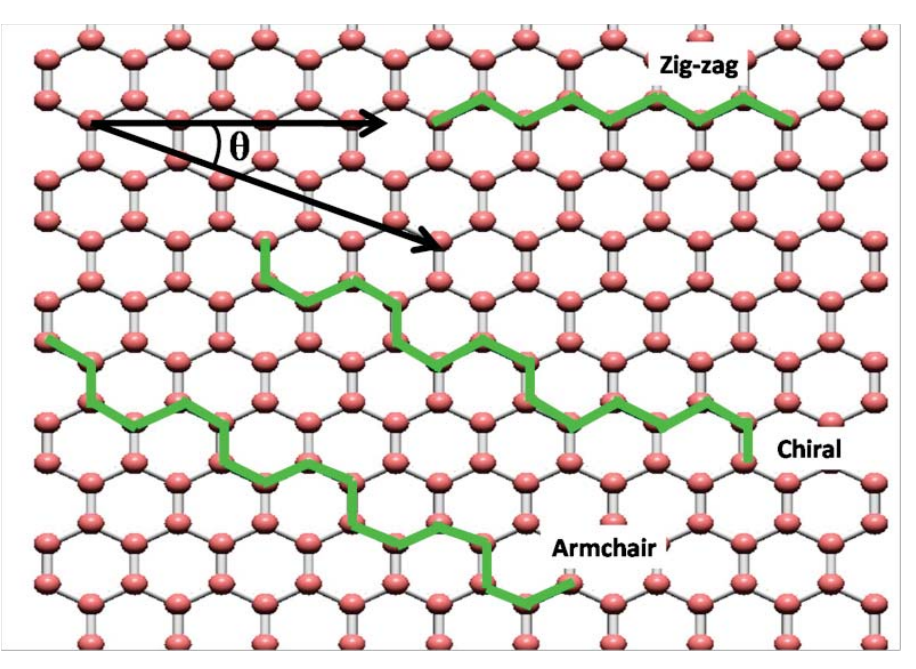

Figure 1.3: A Representation of rolling single walled carbon nanotubes in different ways

The chiral angle $\theta$ is defined as the angle between the chiral vector $C_{h}$ and the so-called "zigzag" direction $(n, 0)$. The integers $(n, m)$ determine $d$ and $\theta$ as follows:

$$
d=\frac{1}{\pi} \sqrt{n^{2}+m^{2}+n m a}, \quad \text { and } \quad \sin \theta=\frac{m \sqrt{3}}{2 \sqrt{n^{2}+m^{2}+n m}}
$$


When graphene sheet is rolled up in one direction of the chiral vector $\boldsymbol{C}_{h}$ one gets a $(n$, $m)$ nanotube. This gives us two special classes of nanotubes and is called "armchair" nanotubes $(n, n)$ and the "zigzag" nanotubes $(n, 0)$. All the others are "chiral" nanotubes $(n, m)$ with $n \neq m$ and $m \neq 0$ (Figure 1.3). The rectangle which is formed in Figure 1.4 defined translation vector $\boldsymbol{T}$ and the chiral vector $\boldsymbol{C}_{h}$ define the unit cell of a nanotube that can be translated in only one direction. The intersection of the vector 'OB' with the first lattice point determines one dimensional lattice vector (T).

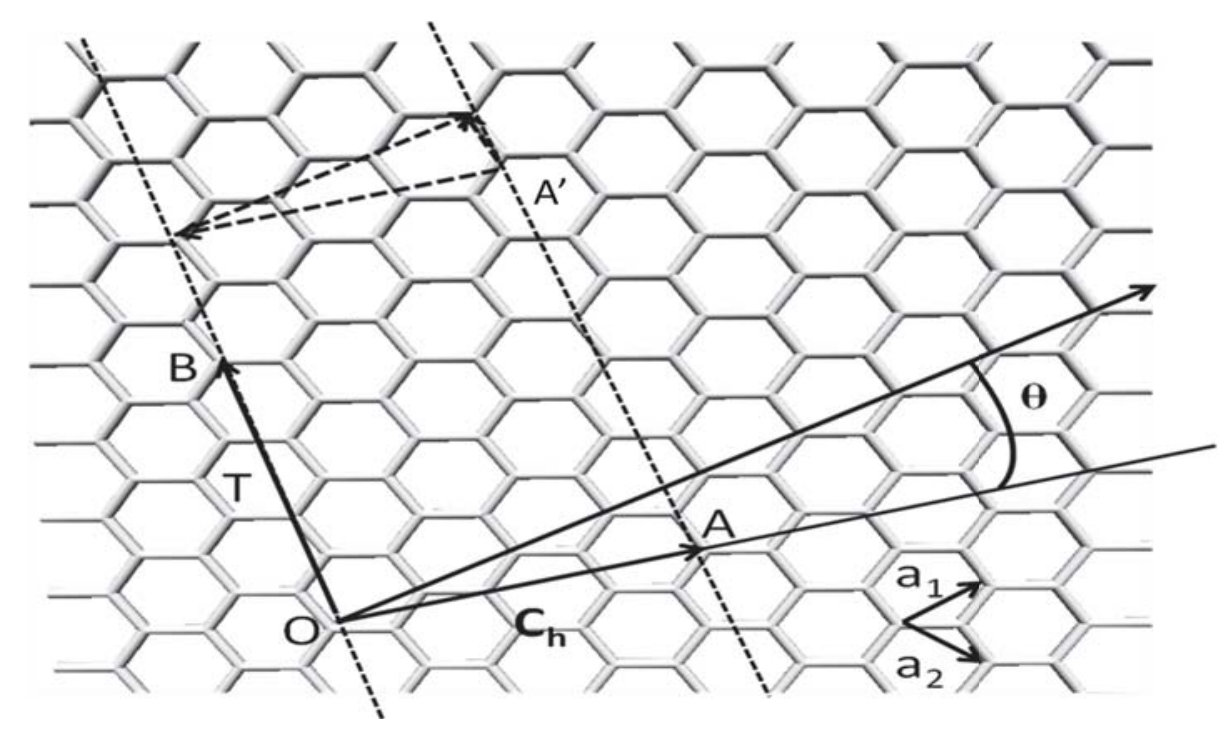

Figure 1.4: A graphene sheet showing different terms used in defining single walled carbon nanotubes

The area enclosed by the vectors ' $\mathbf{O B}$ ' and ' $\boldsymbol{C}_{\boldsymbol{h}}$ ' defines the unit cell of the CNT. A CNT is produced by joining two ends of the chiral vector ' $\boldsymbol{C}_{\boldsymbol{h}}$ ', and the cylindrical shape is made with joining two lines 'OB' and 'AB'. SWNTs are often found in bundles that are formed by a triangular arrangement of individual SWNT [4$6]$. 


\subsubsection{Physical properties of carbon nanotubes}

Carbon nanotubes possess exceptional physical properties. Key points of these physical properties of CNTs are noted below:

- Electrical: Electrical properties of CNTs can be determined by the chiral vector (chirality) and diameter. Depending on chirality CNTs can be metallic, semi metallic or semiconducting. MWCNTs are always conducting. Electron field emission from CNTs and the prototype of CNTs based flat panel displays have been demonstrated [7]. These CNTs have high aspect ratios and they can emit electrons at relatively lower electric field values. This makes CNTs ideal candidate for the electron field emission.

- Optical: CNTs are optically active. Raman spectroscopy of CNTs reveal graphitic order "G" peak and defect order "D" peak. Additionally, SWCNTs show radial breathing mode (RBM) peak corresponding to their diameters [8].

- Thermal: CNTs possess excellent thermal conductivity due to its structure. Composite material of CNTs with epoxy at only a $1 \%$ loading showed excellent thermal conductivity [9].

- Mechanical: CNTs have extraordinary mechanical properties. CNTs have high young's modulus of $1 \mathrm{TPa}$ [10]. This property of CNTs implications for the development of a wide variety of products, such as light weight bullet proof vests, composite material to be used for space elevator and other safety devices etc. [9-11]. 


\subsection{Synthesis methods of carbon nanotubes}

There are numerous methods by which we can produce carbon nanotubes. Among those methods it is possible to grow carbon nanotubes with different properties and with different shapes. Some of the commons methods are:

- Arc discharge

- Laser Ablation

- Chemical vapor deposition (CVD)

- Thermal Chemical vapor deposition

- Plasma Enhanced Chemical Vapor Deposition (PECVD)

\subsubsection{Arc Discharge}

First report on multi-walled carbon nanotubes was published by S. Iijima in 1991 at the NEC Laboratory in Japan. The carbon-soot made by their group used arc discharge method [1]. In arc-discharge, carbon atoms can be evaporated by helium gas plasma. This technique provides the high temperature needed for the evaporation of carbon atoms into a plasma $\left(>3000^{\circ} \mathrm{C}\right)$.

With the arc-discharge method both multi- and single walled carbon nanotubes can be obtained. MWNTs can be grown by optimizing the growth parameters such as the pressure, temperature and the arcing current [12]. A view of an apparatus is shown in Figure 1.5. It consists of two carbon electrodes; the cathode on which the deposit forms is separated from the anode by $\sim 1 \mathrm{~mm}$. During the deposition the anode is consumed. 


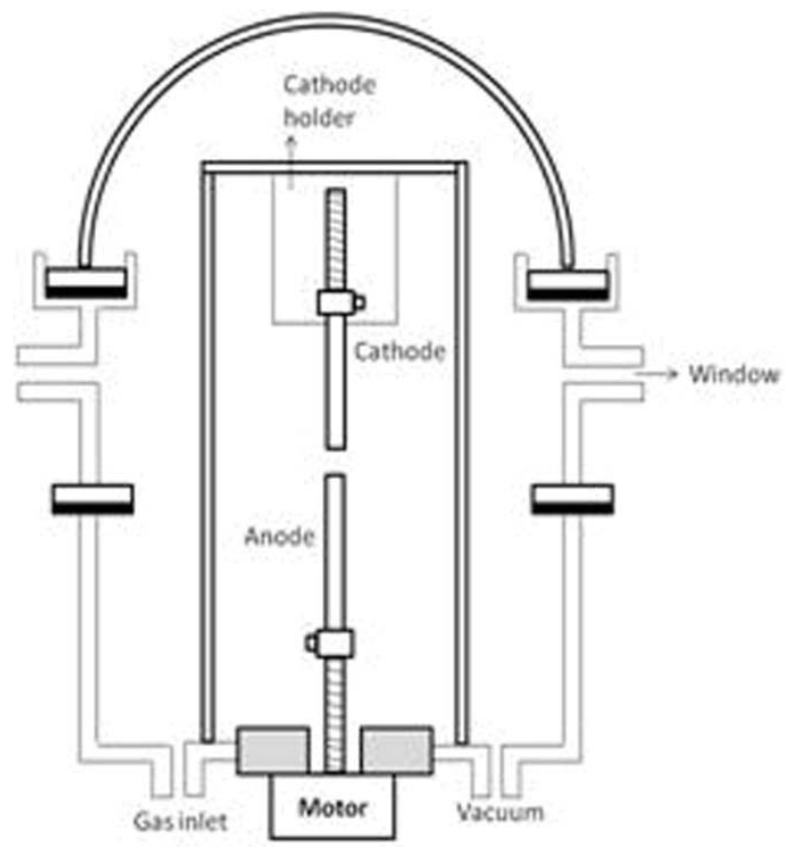

Figure 1.5: Illustration of an arc-discharge apparatus for the production of nanotubes [13].

A voltage of $20-25 \mathrm{~V}$ is applied between the electrodes and the current is between 50-120A. The optimal pressure for producing nanotubes is around 500 torr of He (fullerenes are efficiently produced at a pressure below 100 torr). For the synthesis of MWCNT no catalyst is necessary [13-19].

\subsubsection{Laser Ablation}

The picture of set-up of laser ablation is shown in Figure 1.6. In the laser ablation method a laser evaporates a graphite target material which is placed in an electrical furnace. The growth temperature is at $\sim 1200^{\circ} \mathrm{C}$. The ablated carbon atoms segregate in the form of CNTs and flowing argon gas ( $~ 500$ torr) takes away the 
nanotubes from the high temperature zone to the low temperature collector outside the furnace [20-21].

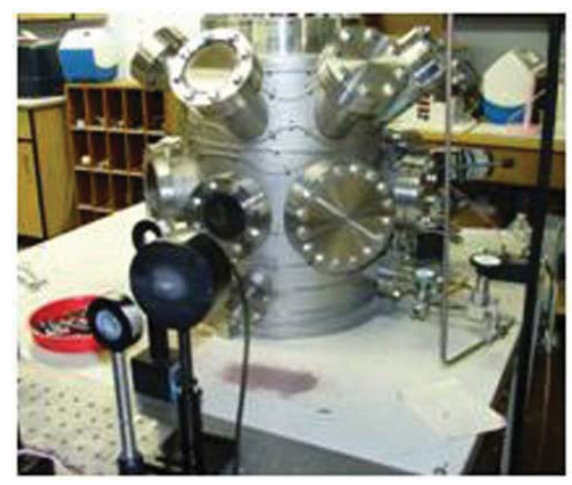

Figure 1.6: Picture of a laser ablation apparatus

If a pure graphite target is used MWNT are produced like in the arc-discharge process but if the target is composed for example of 1.2 atom $\% \mathrm{Co} / \mathrm{Ni}$ with equal amounts of Co and Ni added to the graphite then SWNT are synthesized [21]. High yields with $>70-90 \%$ alteration of graphitic carbon to SWNTs were reported in the condensing vapor of the heated tube. The produced material consists of ropes of SWNT with a diameter between 15 and $20 \mathrm{~nm}$ and up to $100 \mu \mathrm{m}$ or more in length. The average nanotube diameter and the diameter distribution can be adapted by varying the synthesis temperature and the composition of the catalyst [22]. The diameters of the SWNT have strongly peaked distributions. 


\subsubsection{Chemical vapor deposition technique (CVD)}

CVD growth of CNTs is three step processes. First step is the deposition of catalyst on silicon $(\mathrm{Si})$ substrate. There are many methods to deposit catalyst on $\mathrm{Si}$ substrates [23-25]. We use pulsed laser deposition techniques to deposit Ni catalyst films as shown in Figure 1.6.

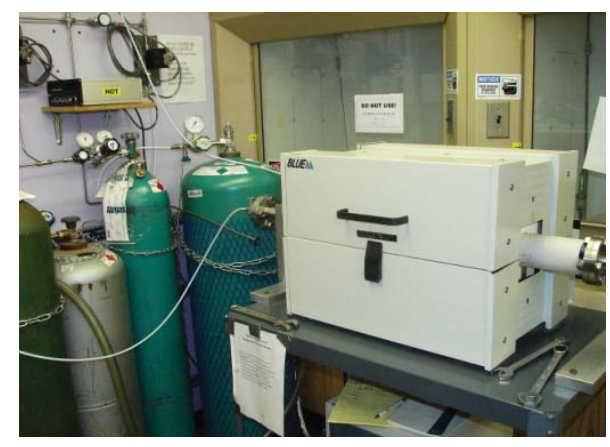

Figure 1.7: Picture of a horizontal CVD furnace used to grow carbon nanotubes

Transition metals such as $\mathrm{Ni}, \mathrm{Fe}, \mathrm{Co}$, Mo are used as catalyst for CNTs growth. Selection of right catalyst is very important for the CNTs growth. With regard to CNTs formation most important properties of the catalysts are their solubility and ability to decompose the carbon containing molecules [26].

The second step is the pretreatment step. In this step the Si substrates with deposited metal particles is transferred to the furnace with high temperature. The catalyst film at this temperature undergoes heat-induced surface diffusion, and collides with each other to form nano-particles. Therefore, pretreatment transforms the 
continuous catalyst film into nano-sized particles that are suitable for CNTs growth [25].

The third step is the actual growth. Picture of CVD chamber in our laboratory is shown in Figure 1.7. For the growth of CNTs precursor gas is supplied into the hot quartz tube. Along with the precursor gas a buffer gas, such as $\mathrm{N}_{2}, \mathrm{H}_{2}$ or $\mathrm{NH}_{3}$, can also be co-supplied to dilute the precursor, if needed. The carbon precursor undergoes catalytic decomposition, and releases carbon atoms upon heating. CNTs are grown on the catalyst nano-particles, and are collected after cooling the chamber to a room temperature in some inert gas environment.

\subsubsection{Plasma enhanced chemical vapor deposition (PECVD)}

Figure 1.8 shows the schematic diagram of PECVD system. In the PECVDmethod, CNTs can be deposited on a Si substrate that is coated with a catalyst film prior to the deposition. Usually one of the following plasmas is used: RF- (radio frequency), MW- (micro wave) or a DC-plasma (direct-current). In our case we use methane $\left(\mathrm{CH}_{4}\right)$ as precursor gas. PECVD is a two step process. In the first step we deposit Ni catalyst on low resistance Si substrates. After that those Si substrates are placed inside the chamber shown in Figure 1.8. 

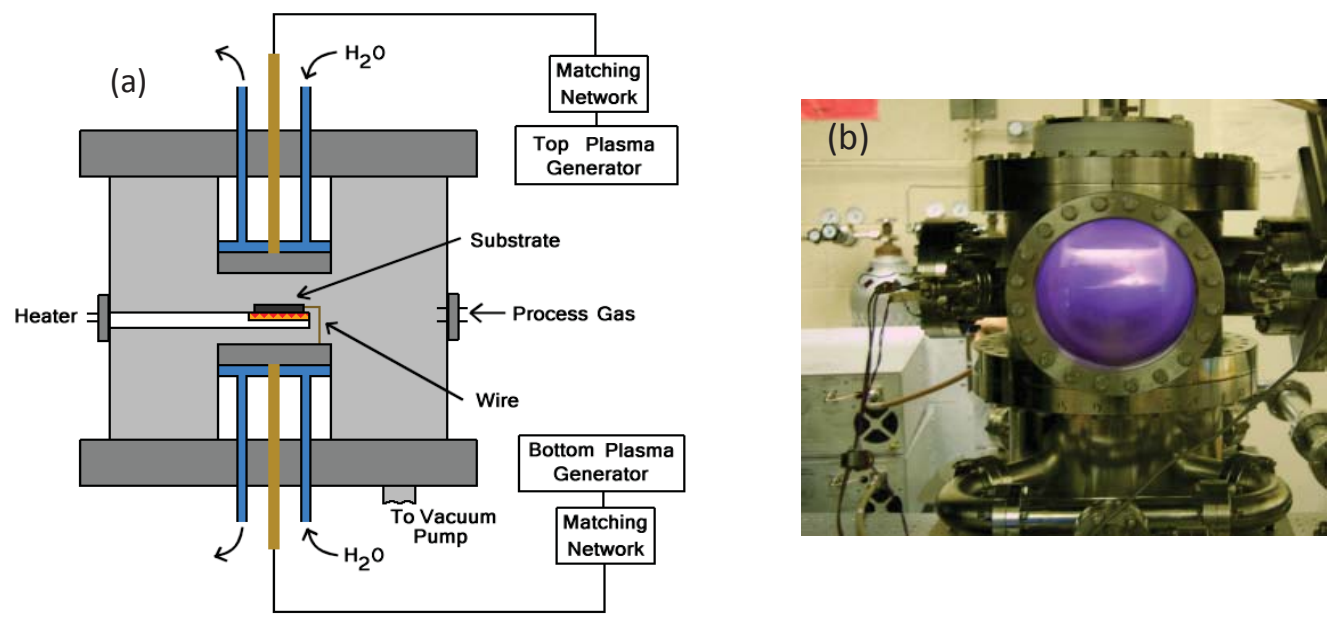

Figure 1.8: (a) Schematic diagram of PECVD system (b) Actual picture of PECVD system [Schematic diagram is reprinted with permission from Ben Ulmen]

The substrate temperature is ramped up to $450^{\circ} \mathrm{C}$ for the growth of nanotubes. The purpose of the top plasma is to decompose the hydrocarbon gases into its components i.e. ions and other free radicals. The bottom plasma provides the bias voltage to the substrates and controlling the ion bombardment on the substrates. We control the top and bottom plasma voltages in our experiments. Bottom plasma also controls the kinetic energy of the growth species which undergoes collisions with the substrates which is held at the some potential by a conducting wire [27, 28]. Some of the standard growth parameters which were used throughout this work are listed in the table below. 
Table 1.1: Growth parameters of MWCNTs

\begin{tabular}{|c|c|c|c|c|c|}
\hline Substrates & Temperature & Flow rate & $\begin{array}{c}\text { Deposition } \\
\text { time }\end{array}$ & $\begin{array}{c}\text { Top } \\
\text { plasma }\end{array}$ & $\begin{array}{c}\text { Bottom } \\
\text { plasma }\end{array}$ \\
\hline L.R. Si & $450^{\circ} \mathrm{C}$ & $100 \mathrm{sccm}$ & 1 hour & $-150 \mathrm{~W}$ & $150 \mathrm{~V}$ \\
\hline
\end{tabular}

\subsection{Growth modes and optimization of growth parameters}

\subsubsection{Effect of flow rate on the growth of CNTs}

One of the critical growth parameters of PECVD is the gas flow rate. We studied the effect of methane flow rate on the growth of carbon nanotubes by PECVD. In this study all other parameters (like temperature, pressure and catalyst thickness) were fixed and we varied the methane flow rate from $10 \mathrm{sccm}$ to $120 \mathrm{sccm}$ in increment of $10 \mathrm{sccm}$.

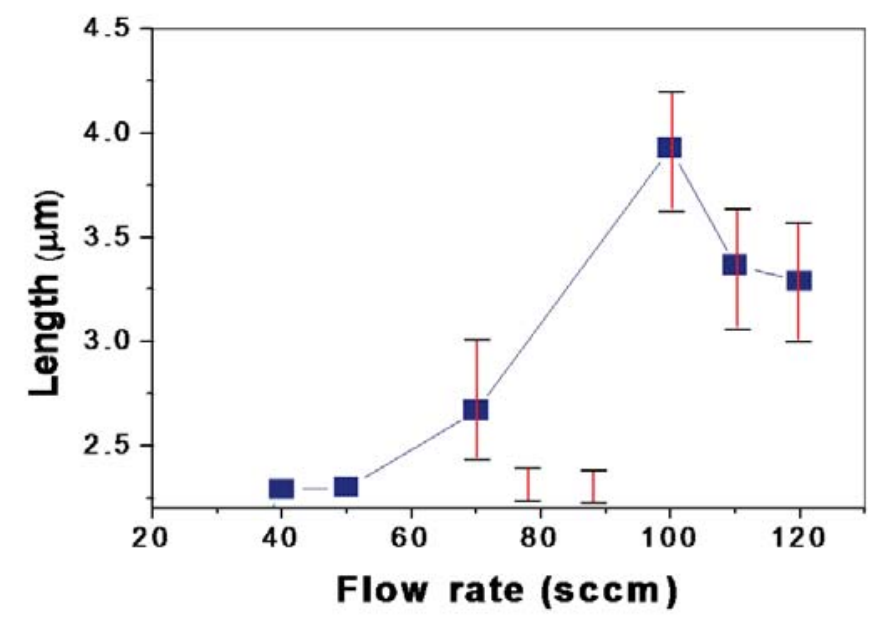

Figure 1.9: Variation of methane flow rate with the length of CNTs

Figure 1.9 shows the effect of methane flow rate on the length of carbon nanotubes. We observe from the graph that as methane flow rate increases, the length 
of MWCNTs increases initially due to more supply of carbon feedstock. But as the flow rate increases further, deposition of amorphous carbon poisons the catalyst and hinders the growth of MWCNTs.

\subsubsection{Role of growth time on length of CNTs}

The exact trend in the growth rate is not known for this growth method. We investigated the effect of growth time with the length of carbon nanotubes. Again we fixed all other parameters and varied the growth time starting from 15 minutes to 90 minutes. The length of the samples was measured using SEM images from S4700FESEM. Figure 1.10 shows the plot of average CNTs length vs growth time of carbon nanotubes.

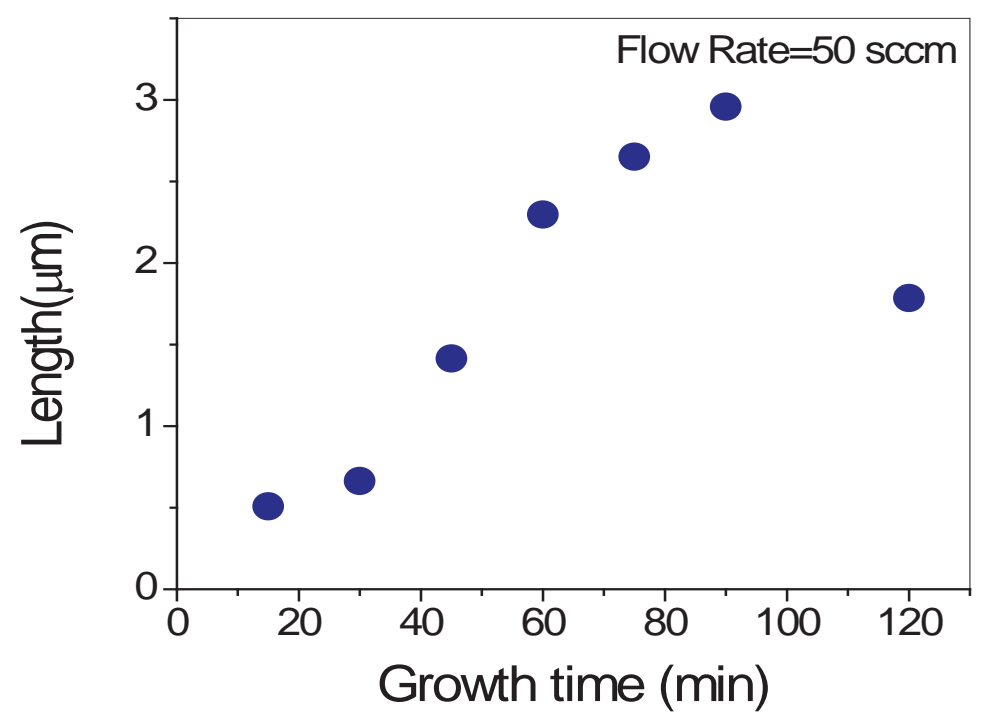

Figure 1.10: Variation of growth time with the length of CNTs 
It can be seen in the figure that the growth rate is not linear. As we increase the growth time, the lengths of the CNTs increase slowly. For a growth duration longer than 90 minutes, the growth rate decreases as catalyst particles become inactive due to amorphous carbon coatings. This indicates that less carbon is absorbing into the catalyst nanoparticles and they are over-coated with amorphous carbon forming a barrier which prevents absorption of any more carbon atoms.

\subsubsection{Growth modes of CNTs}

There are two different growth modes of CNTs [29]. They are as follows:

- Tip growth mode: In this mode the adherence of catalyst nanoparticles to the substrate surface is week. Therefore, carbon precipitation occurs at the bottom and it lifts the catalyst particle as it grows. In this case the tips are decorated with catalyst particle and are called as tip growth mode. It is shown in the Figure 1.11 (a).

- Base growth mode: In this case the adherence of catalyst nanoparticles to the substrate surface is strong. The carbon precipitates from the top surface of the catalyst particle and it continues to grow with the particle attached to the substrate. This growth is called base growth mode. It is shown in Figure 1.11 (b). 


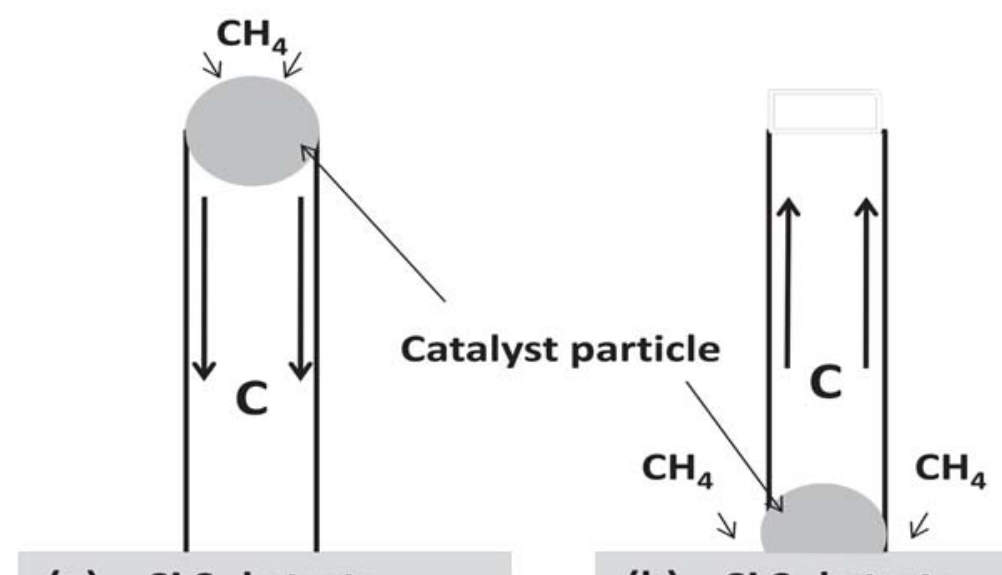

(a) Si Substrate

(b) Si Substrate

Figure 1.11: Illustration of (a) tip growth mode and (b) base growth mode [29]

Details of growth mechanism of PECVD CNTs can be found in Ben Ulmen's master's thesis (Michigan Tech).

\subsection{Potential applications of carbon nanotubes}

Due to their extraordinary properties, CNTs can be used in many applications. Some of them are in microelectronics, field emitters, biosensors, biofuel cells and molecular electronics. Here applications related to the scope of this thesis are discussed.

\subsubsection{Field Emission}

One of the most popular applications of CNTs as utilizing the small size of SWNTs is field electron emitters. Because of the tiny cross sections of SWNTs, the electrical fields around the tips are high value when a potential is applied between 
SWNTs and the ground. This high field facilitates the electron tunneling process. This kind of emitters can be used to extract electrons to generate lights via florescence, and even X-ray (portable and high resolution X-ray machine). Being an attractive source for electrons as compared to thermionic emission, field emission is a quantum phenomenon. When sufficiently high electric field is applied then the electrons near the Fermi level have enough energy to tunnel into the energy barrier and escape to the vacuum level [30-33].

\subsubsection{Electrochemical biosensors}

MWNTs are chemically inert, especially when no defects are present. This makes them chemically stable and biologically compatible. CNTs have ability to promote fast electron transfer with different redox active proteins such as glucose oxidase [34] and horseradish peroxidase [35]. For practical use of CNTs in biomedical applications the ability to functionalize these CNTs (without changing their properties) with biomolecules is very important. Various techniques have been demonstrated for functionalization of CNTs with biomolecules. These CNTs are important functional material for development of biosensors but device fabrication and design also play an important role in controlling biosensor performance. This work studies the CNTs based biosensors in detail and is described in chapter 4 . 


\subsubsection{Bio-fuel Cells}

Since MWNTs are chemically stable and biologically compatible so they can be used for the detection of biological molecules. In this work MWNTs were used for biosensors and biofuel cells. Chapter 5 describes the detailed study of CNTs based biofuel cells. These examples are only a small subset of potential applications due to the small sizes of MWNTs [35-37].

\subsection{Organization of the thesis}

Chapter 1 is an Introduction to CNTs, their applications and optimization of growth parameters.

Chapter 2 briefly described all the experimental techniques used in this work.

Chapter 3 provides a description of the field emission measurements of the CNTs and our new discoveries on improving the field emission characteristics of CNTs.

Chapter 4 compiles our investigation on using CNTs as glucose biosensors. Detailed in this chapter are introduction to the biosensors and the experimental procedures used to obtain successful functionalization. This chapter also contains the description of amperometric response and the effect of electrode area on the performance of glucose biosensors. 
Chapter 5 describes a novel concept of energy sensing, generation, and storage (SGS) system that integrate glucose biosensors, bio fuel cells (BFCs), and supercapacitors into a potentially implantable device system. The development of a bio-fuel cells (BFCs) using our CNT samples is then described. We have investigated the effects of miniaturizing the device area of the BFCs. Finally, the use of our CNT samples in supercapacitors will be discussed.

Chapter 6 concludes the thesis and incorporates a discussion on possible future experiments to improve and consolidate the work on the proposed energy SGS system. The future work on electron field emission, glucose biosensors, BFCs and supercapacitors are provide at the end of Chapter 3, 4, and 5, respectively.

\subsection{Conclusion}

Brief introductions on CNTs were discussed at the beginning of this chapter. They are cylinders of hexagonal graphene sheets and they can be either SWNTs or MWNTs. Different growth techniques and growth modes were also discussed. The potential application of CNTs in field emission, biosensors and biofuel cell was also presented in this chapter. Finally, this chapter provides the organization of the thesis work.

\subsection{References}

1. S. Ijima, Helical microtubules of graphitic carbon. Nature (London) 1991,354, 56. 
2. S. Iijima, T. Ichihashi. Single-shell carbon nanotubes of 1-nm diameter. Nature (London) 1993, 363, 603.

3. M. S. Dresselhaus, G. Dresselhaus, P. Avouris. Carbon nanotubes: Synthesis, structures, nanotubes, Academic, San Diego (1996)

4. M. S. Dresselhaus, G. Dresselhaus, R. Saito. Carbon fibers based on C-60 and their symmetry, Phys. Rev. B 1992, 45, 6234.

5. M. S. Dresselhaus, G. Dresselhaus, P. C. Eklund. Science of fullerenes and carbon nanotubes, Academic, San Diego (1996).

6. M. Meyyappan. Carbon nanotubes: Science and applications, 2005, CRC press ISBN 0849321115, 9780849321115, 289

7. W. B. Choi, D. S. Chung, H. Y. Kim, Y. W. Jin, I.T. Han, Y. H. Lee, J. G. S. Park, J. M. Kim. Fully sealed, high-brightness carbon-nanotube field-emission display, Appl. Phys. Lett., 1999, 75, 3129.

8. H. katura, Y. Kumazawa, Y. Maniwa, I. Umezu, S. Suzuki, Y. Ohtsuka, and Y. Achiba. Optical properties of single-walled carbon nanotubes, Synthetic Metals, 1999, 103, 2555.

9. S. Berber, Y. K. Kwon, and D. Tománek. Unusually high thermal conductivity of carbon nanotubes, Phys. Rev. Lett., 2000, 84, 4613.

10. K. Suzuki, S. Nomura. On elastic properties of single-walled carbon nanotubes as composite reinforcing fillers, Journal of Composite Materials, 2007, 41, 1123. 
11. B. I. Yakobson, C. J. Brabec, J. Bernholc. Nanomechanics of carbon tubes: Instabilities beyond linear response, Phys. Rev. Lett., 1996, 76, 2511.

12. J.-P. Salvetat, J.-M. Bonard, N. H. Thomson, A. J. Kulik, L. Forr'o, W. Benoit, L. Zuppiroli. Mechanical properties of carbon nanotubes, Appl. Phys. A, 1999, $69,255$.

13. W. A. de Heer, J.-M. Bonard, T. Stöckli, A. Châtelain, L. Forró, D. Ugarte. Carbon nanotubes films: electronic properties and their application as field emitters Z. Phys. D, 1997, 40, 418.

14. T. W. Ebbesen, P. M. Ajayan. Large-scale synthesis of carbon nanotubes, Nature, $1992,358,220$.

15. Y. Saito, M. Inagaki. Optical emission studies on chemical species in an arc flame of fullerene/metallofullerene generator, Jap. J. Appl. Phys., 1993, 32, L954.

16. C. Journet, W. K. Maser, P. Bernier, A. Loiseau, M. L. de la Chapelle, S. Lefrant, P. Deniard, R. Lee, J. E. Fischer. Large-scale production of single-walled carbon nanotubes by the electric-arc technique, Nature, 1997, 388, 756.

17. W. Krätschmer, L. D. Lamb, K. Fostiropoulos, D. R. Huffman. Solid C60: a new form of carbon, Nature, 1990, 347, 354.

18. P. A. Ajayan, S. Iijima. Smallest carbon nanotubes, Nature, 1992, 358, 23. 
19. V. P. Dravid, X. Lin, Y. Wang, X. K. Wang, A. Yee, J. B. Ketterson, R. P. H. Chang. Buckytubes and derivatives: Their growth and implications for buckyball formation, Science, 1993, 259, 1601.

20. S. Iijima. Growth of carbon nanotubes, Mater. Sci. Eng. B, 1993, 19, 172.

21. T. Guo, P. Nikolaev, A. G. Rinzler, D. Tomanek, D. T. Colbert, R. E. Smalley. Self-assembly of tubular fullerenes, J. Phys. Chem., 1995, 99, 10694.

22. A. Thess, R. Lee, P. Nikolaev, H. Dai, P. Petit, J. Robert, C. Xu, Y. H. Lee, S. G. Kim, A. G. Rinzler, D. T. Colbert, G. E. Scuseria, D. Tomanek, J. E. Fischer, R.E. Smalley. Crystalline ropes of metallic carbon nanotubes, Science, 1996, $273,483$.

23. R. Saito, G. Dresselhaus, M. S. Dresselhaus. Physical properties of carbon nanotubes, Imperial College Press (London) 1998

24. A. Moisala, A. G. Nasibulin, E. I. Kauppinen. The role of metal nanoparticles in the catalytic production of single-walled carbon nanotubes-a review J. Phys.: Condens. Matter, 2003, 15, S3011.

25. V. K. Kayastha, Y. K. Yap, S. Dimovski, Y. Gogotsi. Controlling dissociative adsorption for effective growth of carbon nanotubes, Appl. Phys. Lett., 2004, 85,3265 . 
26. V. K. Kayastha, Y. K. Yap, Z. Pan, I. N. Ivanov, A. A. Puretzky, D. B. Geohegan. High-density vertically aligned multiwalled carbon nanotubes with tubular structures Appl. Phys. Lett., 2005, 86, 253105.

27. J. Emsley. The Elements, Oxford: Clarendon, 1991, 264.

28. Ben Ulmen, M.S. Thesis, Physics. Michigan Technological University. (2007).

29. J. Menda, B. Ulmen, L. K. Vanga, V. K. Kayastha, Y. K. Yap, Z. Pan, I. N. Ivanov, A. A. Puretzky, D. B. Geohegan. Structural control of vertically aligned multiwalled carbon nanotubes by radio-frequency plasmas, Appl Phys Lett 2005, 87, 173106-1.

30. A. M. Cassell, J. A. Raymakers, J. Kong, H. Dai. Large scale CVD synthesis of single-walled carbon nanotubes, J. Phys. Chem. B, 1999, 103, 6484.

31. B. Ulmen, V. K. Kayastha, A. DeConinck, J. Wang, Y. K. Yap. Stability of field emission current from various types of carbon nanotube films. Diamond Related Mater., 2006, 15, 212.

32. Y. Cheng, O. Zhou. Electron field emission from carbon nanotubes. C. R. Physique 2003, 4, 1021.

33. W. A. deHeer, A. Chatelain, D. Ugarte. A carbon nanotube field emission electron source. Science 1995, 270, 1179. 
34. K. Yamamoto, G. Shi, T. S. Zhou, F. Xu, J. M. Xu, T. Kato, J. Y. Jin, L. Jin. Study of carbon nanotubes-HRP modified electrode and its application for novel online biosensors, Analyst, 2003, 128, 249.

35. G. Wang, J. J. Xu, H. Y. Chen. Interfacing cytochrome c to electrodes with a DNA-carbon nanotube composite film, Electrochem. Commun., 2002, 4, 506.

36. W. R. Yang, P. Thordarson, J. J. Gooding, S. P. Ringer, F. Braet. Carbon nanotubes for biological and biomedical applications, Nanotechnology, 2007,18, 412001

37. J. Wang. Carbon-Nanotube Based Electrochemical biosensors: A review Electroanalysis, 2005, 17, 7.

38. F. Davis, S. P. J. Higson. Biofuel cells-recent advances and applications, Biosensors and Bioelectronics, 2007, 22, 1224.

39. M. J. Cooney, V. Svoboda, C. Lau, G. martin, S.D. Minteer. Enzyme catalysed biofuel cells, Energy Environ. Sci., 2008, 1, 320. 
Chapter 2

\section{Investigation methods}

\subsection{Electron microscopy}

When the electron beam with high energy is impinged upon sample surface then a wide range of secondary signals are formed each of which can give interesting information of the sample (Figure 2.1). A scanning electron microscope (SEM) analyzes the backscattered electrons (BSE), secondary electrons (SE), and characteristic X-rays (EDX).

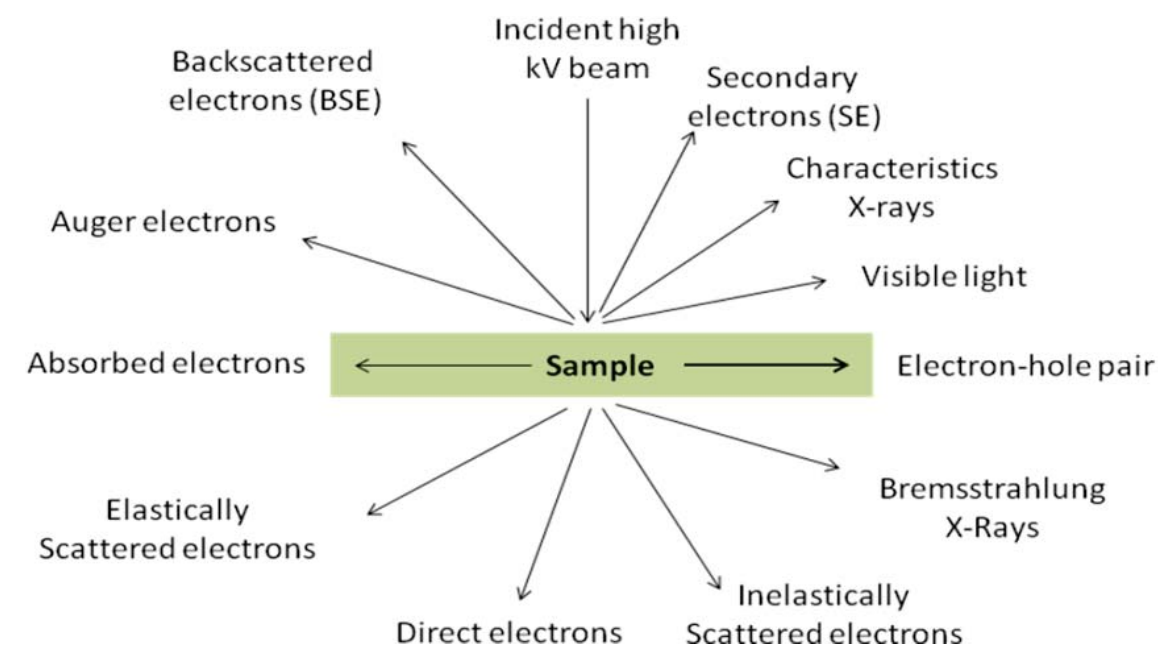

Figure 2.1: Signals generated when high energy electron beam interacts with a thin specimen [1].

When the sample is ultrathin enough then transmitted electrons such as elastically scattered electrons can be analyzed in a transmission electron microscope (TEM) to give a much higher atomic scale resolution [1]. 


\subsubsection{Scanning electron microscopy (SEM)}

SEM can provide high resolution and large depth of field. In SEM an electron beam generated by field emission is irradiated on the specimen. The backscattered or secondary electrons coming out from the specimen are counted with a detector and are imaged on a screen. The different properties of the secondary and backscattered electrons from the specimen can be reveled in SEM. Energy of the secondary electrons is low and they are attracted with a positively charged grid on the secondary electron detector. The energy of the backscattered electrons is too high to be attracted by the grid. The secondary electrons provide topographic information of the morphology of the specimen. The energy of the backscattered electrons is high enough to create electron-hole pairs in a semiconductor detector. Hence secondary electrons provide information about the chemical composition of the specimen.

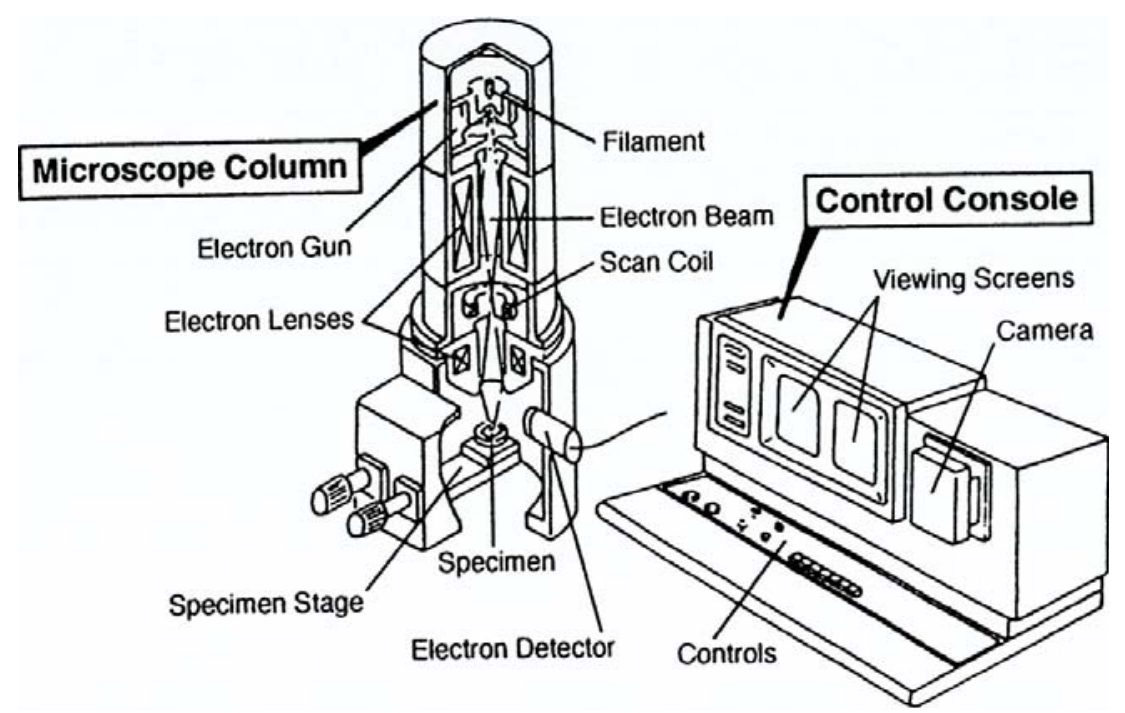

Figure 2.2: Two major components of SEM: microscope column and control console. Reprinted with permission from [1] 
Important parts of SEM are shown in Figure 2.2 such as microscope column and control console. The microscope column consists of electromagnetic lenses and an electron gun. The electromagnetic lenses are used to control the paths of electrons moving in the vacuum tube [2]. Example of SEM image of as grown CNTs obtained by Hitach S-4700 SEM is shown in Figure 2.3.

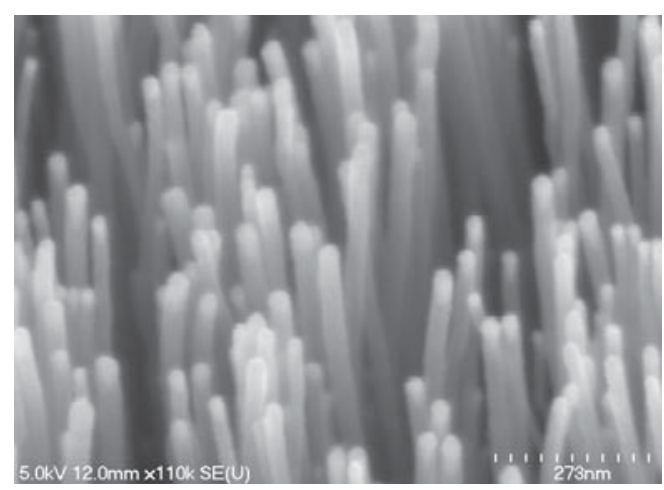

Figure 2.3: SEM image of as grown carbon nanotubes

Morphology imaging of as grown MWCNTs is easy. MWCNTs are vertically aligned and estimation of their diameters can also be made using SEM. Our MWCNTs have Ni nanoparticles on their tips which suggest that they followed the tip growth mode.

\subsubsection{Transmission electron microscopy (TEM)}

TEM as the name suggests is based on imaging elastically scattered and transmitted electrons from an ultrathin specimen. TEM has advantage of much higher resolution down to the atomic scale. The imaging optics in a TEM is similar to that of an optical microscope [2]. 


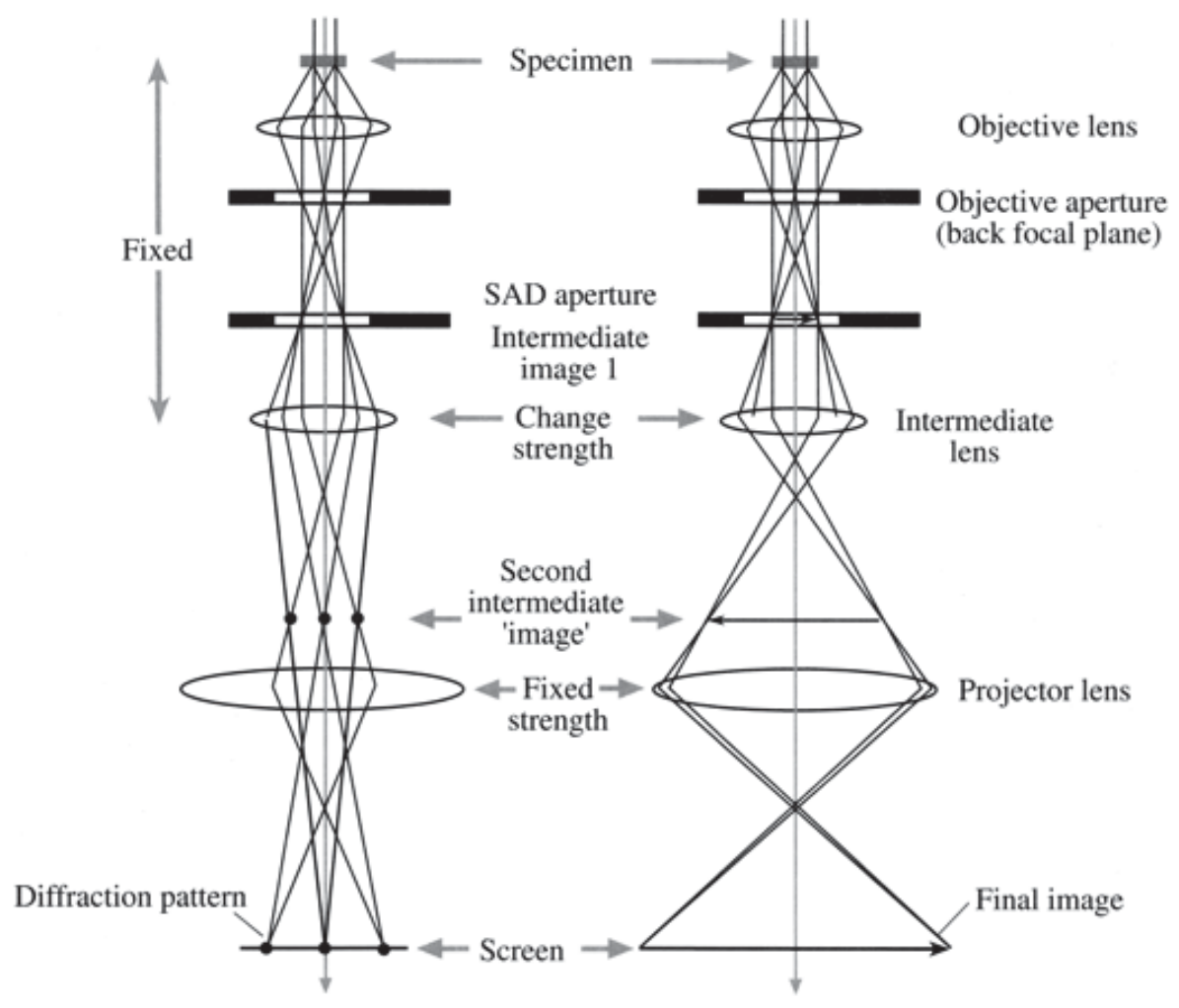

Figure 2.4: The two basic operations of a TEM: projecting the diffraction pattern on the viewing screen (left) or projecting the image on the screen (right). The intermediate lens selects the back focal of the objective lens as its object. Reprinted with permission from [3]

The operation of the electromagnetic lens allows the imaging of the object in the corresponding image plane by focusing ultra-thin beam of electrons starting with different directions from individual object points to the corresponding image points (Figure 2.4). As shown in Figure 2.4 on left, in the back focal plane of the objective all electrons are focused to one point, which started in the same direction from any point in the object which leads to formation of the diffraction pattern of the object. 


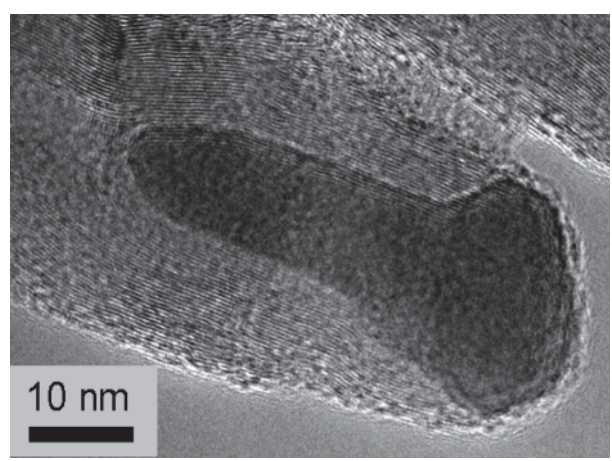

Figure 2.5: TEM image of as grown carbon nanotubes

A bright field image contrast can be obtained by arranging a contrast aperture in the back focal plane to eliminate all electrons, which are scattered and diffracted, respectively into large angles. Depending on the setting of the lenses one may image the back focal plane as well as the image plane of the objective lens on the image plane of the projector lens. Thus one obtains either the diffraction pattern or the image of the specimen on the viewing screen. The image contrast in TEM arises mainly from the elastic scattering process [3]. Example of TEM image of CNTs obtained by is shown in Figure 2.5. It can be that CNT is multi-walled with nickel nanoparticles on top.

\subsection{Raman spectroscopy}

When light beam is incident on molecules on a surface, it is scattered in different directions. A large portion of the incident beam of light is scattered elastically, which mean scattered light rays possess photons of energy and wavelength same as those of incident photon. This process of elastic scattering is known as Rayleigh scattering. 
Only very few scattered photons $\left(\sim 1\right.$ in $10^{7}$ photons $)$ have different energy and wavelength. When light is incident on a molecule, the incident light will interact with the electric dipole of that molecule. The photon of light (light quantum), excites one of the electrons in the molecule into a virtual state. Since this virtual state is unstable so the electron decays back to its lower state releasing another photon. This process occurs in few femto-seconds. This kind of scattering is inelastic where the incident light photon either gives or takes energy from scattering molecules is known as Raman scattering. Rayleigh and Raman scatterings can be represented by electron transitions as shown in Figure 2.6.

A fraction of incident light is scattered inelastically by the specimen. Intensity and wavelength of this in-elastically scattered light is measured by Raman spectroscopy. Inelastically scattered light occurs has a different wavelength when compared from the wavelength of the incident light. Molecular vibrations shift the energies of incident light thereby producing in-elastically scattered light which has a different wavelength. Difference in the energies of incident light photon and inelastically scattered photon corresponds to vibration energy of scattering chemical bond or molecule. This difference or shift in energy is unique for different bonds or molecules [4]. 


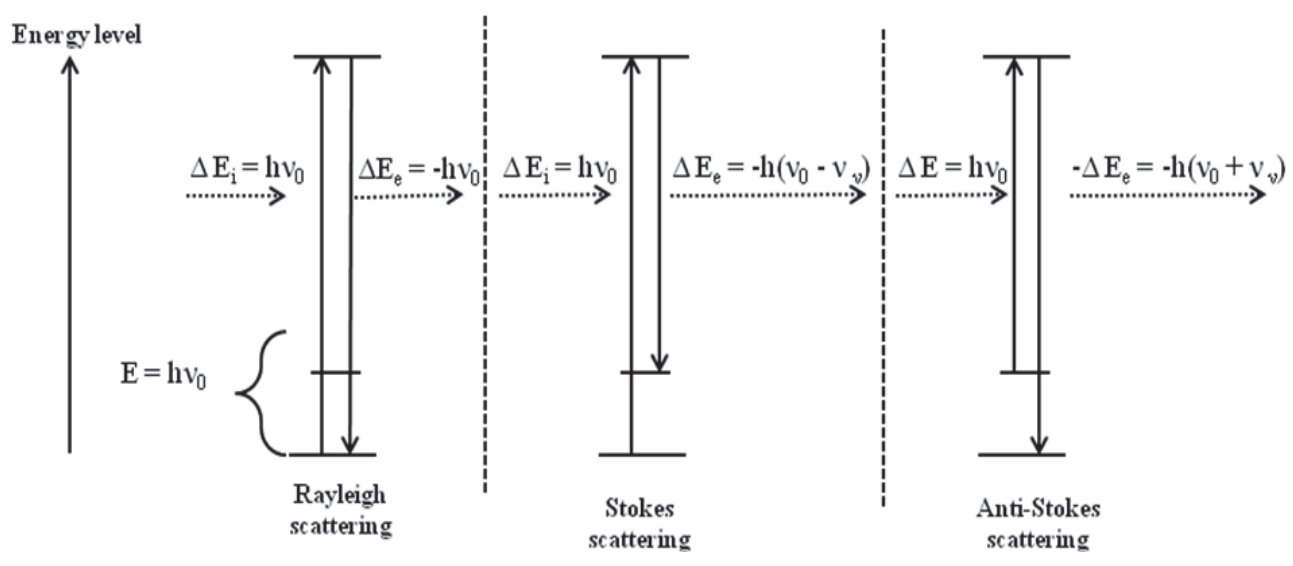

Figure 2.6: Different kinds of scattering of light photons [4]

The plot of intensity of scattered light versus the Raman shift is known as a Raman spectrum. Raman shift can be given by

$$
\begin{aligned}
\text { Raman shift } & =\text { energy difference between incident and scattered photon } \\
& =\text { energy difference between initial and final vibrational levels }
\end{aligned}
$$

of scattering molecule $v\left(\mathrm{~cm}^{-1}\right)=1 / \lambda_{\text {incident }}-1 / \lambda_{\text {scattered }}$

Energy absorbed by molecules, which is very less, is dissipated as heat.

\subsubsection{Stokes and Anti-Stokes Scattering}

When photon energy of the scattered light is less than that of the incident photon, this process is called Stokes scattering. When the incident light photon is scattered from a molecule which is already in excited state, the molecule will be further excited by absorbing the incident photon. When the molecule relaxed to the 
ground state, the scattered light photon has energy higher than the incident light photon which is known as Anti-Stokes scattering [4].

\subsubsection{Raman spectroscopy of multi-walled carbon nanotubes (MWCNTs)}

Raman spectrum from a CNT sample gives a lot of information about their structures such as diameters, type (SWNTs or MWNTs) and chirality of the nanotubes. It is because CNTs with different diameters, types and quality cause the incident photon to scatter differently. Their energy after scattering is determined by the CNTs. So the intensity and the location of the characteristic peaks on the spectra (Raman shift) is different for different types of nanotubes. Hence, the wavenumber and intensity of the peaks present in the Raman spectrum gives information about CNTs. Among the six vibrational modes in graphite only the two $\mathrm{E}_{2 \mathrm{~g}}$ vibrational modes are Raman active.
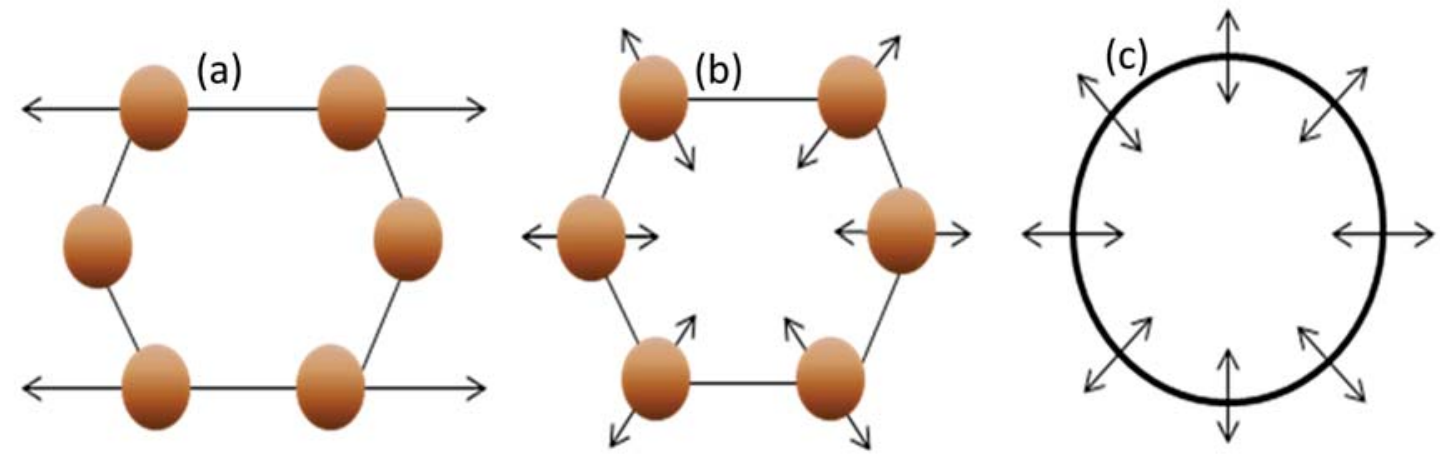

Figure 2.7: "Vibration modes in Raman spectroscopy of carbon nanotubes: Hexagonal shape formed by carbon atoms shows an in-plane vibration (a) and (b). The arrows show the direction of vibrational motion of carbon atoms. (a) $G$-band $\left(E_{2 g}\right)$ mode (common to graphite) (b) D-band mode (common to disorder carbons and polycrystalline graphite) (c) RBM mode (unique to SWNTs, fullerenes)" [5] 
Raman spectrum of a MWNT sample consists mainly of two peaks. The first peak around wave number $1350 \mathrm{~cm}^{-1}$ is known as defect induced peak or D-peak in short. Defect peak arises due to in-plane substitutional hetero-atoms, defects at the grain boundaries, defects in CNTs walls and deposited amorphous carbon etc. Higher intensity of D-peak indicates that the tubes are more defective. Possible Raman vibrational modes in carbon nanotubes can be seen Figure 2.7. The second peak is graphitic or G-peak for short is around wavenumber $1590 \mathrm{~cm}^{-1}$. The G peak represents the graphitic order and crystallinity of the CNTs walls. G-peak occurs due to the inplane or tangential vibration of carbon atoms [5-6].The higher the quality of the CNTs, the higher is the ratio of intensities of G-peak upon D-peak.

In SWCNTs radial breathing mode (RBM) is induced due to the vibration of carbon atoms along radial directions. In MWCNTs it is absent due to cancellation by vibration of concentric tubes. Radial breathing mode can tell us about the diameter of SWCNTs. The Raman shift is related to the diameter of SWCNT as

$$
v\left(\mathrm{~cm}^{-1}\right)=248 / \mathrm{d}(\mathrm{nm})
$$

Again, mathematically also it can be seen that due to the large diameters, the RBM Raman shift is very small in MWNTs. 


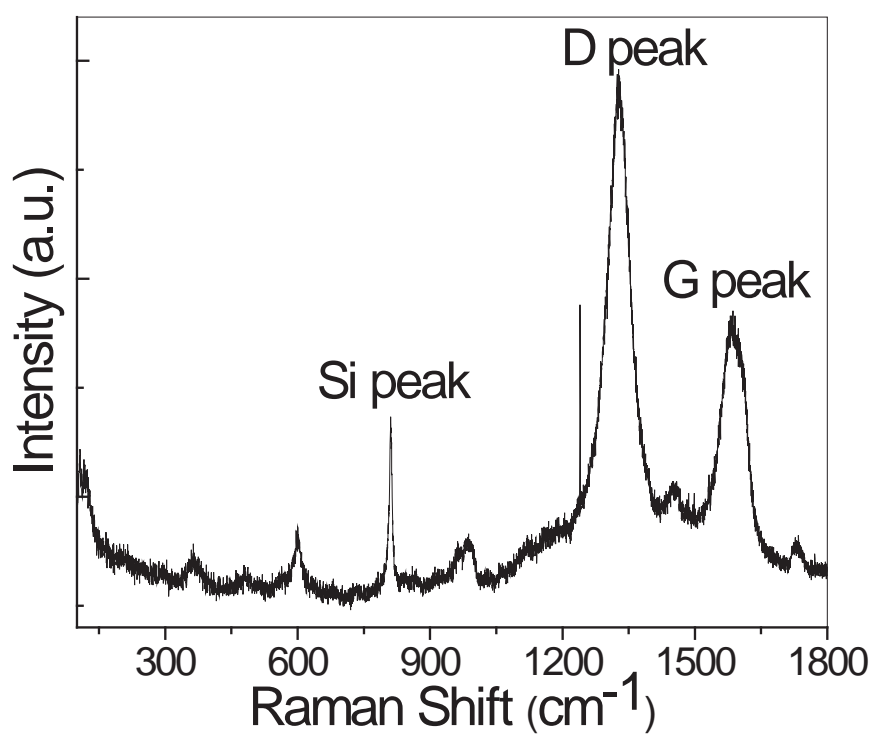

Figure 2.8: Raman spectrum of multi-walled CNTs.

The characteristic Raman spectrum of SWCNTs is the presence of RBM. Moreover, the G-peak is very narrow and intense with splitting. So the presence of RBM mode and intense G peak with splitting confirms the presence of SWCNTs in specimen. A typical spectrum of MWCNTs can be seen in Figure 2.8. In the Raman spectra, broad G peak represents large diameter distribution in the CNT specimen. RBM peaks can be found in Raman spectra of SWCNTs. Different diameters of CNTs have different magnitude of scattering so they have different Raman shift and make the peaks broad [6-9]. 


\subsection{Fourier Transform Infrared (FTIR) Spectroscopy}

FTIR is the favorable method of infrared spectroscopy. In FTIR spectroscopy, infrared radiation is allowed to pass through a sample. Some of the radiation is transmitted and some of the infrared radiation is absorbed by the sample. The resultant spectrum corresponds to the molecular fingerprint of the sample and provides information about the different chemical groups and chemical bonds present in the sample. Two molecular structures never generate the same infrared spectrum like fingerprints are unique for every person [10]. This is why FTIR spectroscopy is practical for many types of investigation.

\subsubsection{FTIR of CNTs}

An example of FTIR spectrum obtained is shown in Figure 2.9.
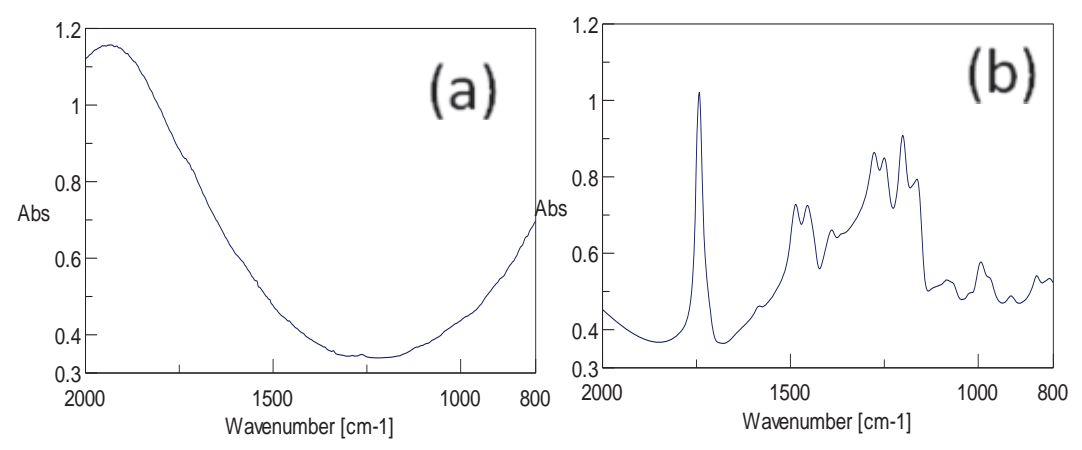

Figure 2.9: FTIR spectra of (a) the as grown and (b) electrochemically treated VA-MWCNTs

Figure 2.9 compares the FTIR spectra of as grown CNTs (Figure 2.8(a)) and electrochemically treated CNTs (Figure 2.9 (b)) which have attached with the $-\mathrm{COOH}$ group. The difference can be clearly seen in Figure 2.9. 


\subsection{Cyclic Voltammetry (CV)}

Cyclic Voltammogram (CV) is an extremely useful technique in electrochemical characterization. $\mathrm{CV}$ is obtained by using a potentiostat to vary the voltage between electrodes dipped in an electrolyte solution and recording the current output. In electrochemistry $\mathrm{CV}$ is extensively used for studying the oxidation and reduction properties of various chemicals and interfaces.

\subsubsection{What is CV?}

In a typical $\mathrm{CV}$ experiment, linearly varying potential is applied using a potentiostat at a known rate. The faradic current output or response is measured. Current obtained from a redox reaction is known as Faradic current. In the forward scan potential is linearly varied starting from an initial value to a limiting value at a known scan rate. Scan rate $(v)$ is defined as the rate of change of potential with time. When potential reaches its limiting value, direction of the potential scan is reversed. In the reverse scan again potential is linearly scanned back to its starting value.

Electrolyte provides a medium (usually liquid) for electrochemical reactions to occur at the interface of electrolyte and electrode. Conduction through electrolyte is ionic which involves movement of ions in the medium. 


\subsubsection{Electrochemical cell and electrodes used in CV experiments}

In a CV measurement three electrodes and an electrolyte is used. The first electrode is called working electrode. At this electrode the electrochemical phenomena being examined takes place. A number of noble metal electrodes and carbon electrodes are available for use as working electrode in CV measurements, including platinum, gold, silver and glassy carbon. Carbon electrode is useful in both aqueous and non aqueous solutions (for both oxidation and reduction reactions). In this work we use CNT-PMMA matrices as working electrodes.

The second electrode is known as the reference electrode. Potential of the reference electrode is constant. Therefore, potential of reference electrode is taken as the standard value. Potentials of the other electrodes in the cell can be measured by comparing it with reference electrode.

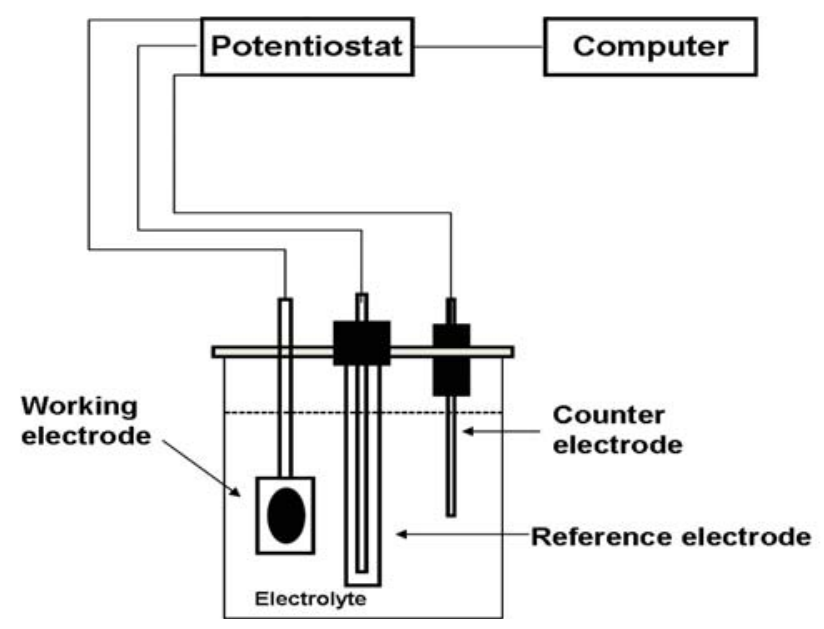

Figure 2.10: Typical cell and electrode setup used for cyclic voltammetry 
The ideal reference electrode should be reversible and obey the Nernst equation. Its potential should not change much with time. In our case we use silver $(\mathrm{Ag}) / \mathrm{silver}$ chloride $(\mathrm{AgCl})$ as a reference electrode.

The third electrode is known as the counter electrode. It acts as a source or sinks for electrons. This allows the current to flow from the external circuit through the cell. Platinum $(\mathrm{Pt})$ wire or Gold $(\mathrm{Au})$ target is used as a counter electrode throughout this work. The set up of these electrodes in a typical electrochemical cell for $\mathrm{CV}$ measurements is shown in Figure 2.10. A Potentiostat is used to control the voltage and its rate of change applied on the working electrode and measures the current of the working electrode. The Potentiostat applies a desired potential to the working electrode with respect to reference electrode. The current flows between the working and the counter electrode are collected as the CV signals.

\subsubsection{Measurements}

The basic shape of the current-potential curve for a CV measurement is shown in Figure 2.11. Consider a fundamental reversible redox reaction between oxidized species $\mathbf{O}$ and its reduced form $\mathbf{R}$ involving $n$ electrons:

$$
O+n e^{-} \leftrightarrow R
$$




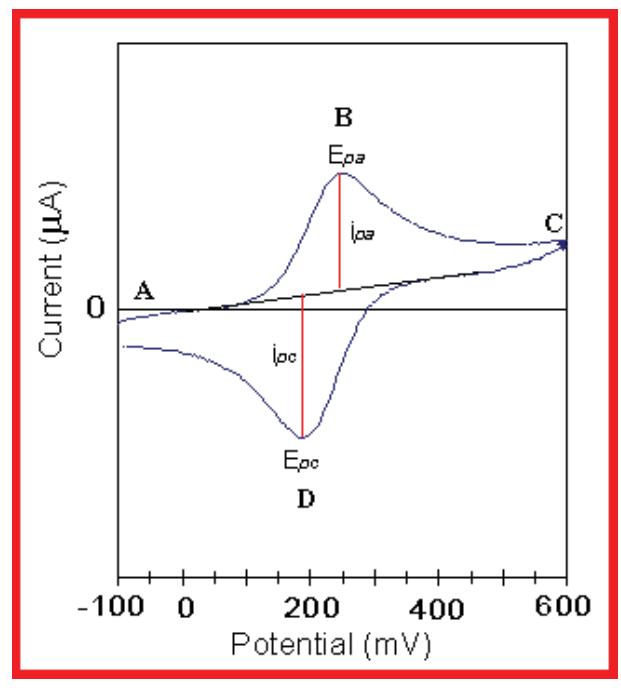

Figure 2.11: Current potential curve for a cyclic voltammetry measurement.

The relation between the equilibrium concentration of $\mathrm{O}$ and $\mathrm{R}$ species and the potential is governed by Nernst equation:

$$
E=E^{0^{\prime}}+\left(\frac{R T}{n F}\right) \ln \left(\frac{C_{O}}{C_{R}}\right)
$$

where $\mathrm{E}$ is the applied potential, $\mathbf{E}^{\mathbf{0}^{\prime}}$ is the redox potential for the redox couple, $\mathbf{C}_{\mathbf{O}}$ is surface concentration of oxidized species and $\mathbf{C}_{\mathbf{R}}$ is the surface concentration of reduced species adjacent to the electrode.

The basic shape of the cyclic voltammogram for such a redox couple is shown in Figure 2.11. When the applied potential of the working electrode is more positive than that of a redox couple presents in the solution, the corresponding species may be oxidized (i.e. loss of electrons from the solution to the electrode) and produces an anodic current. Again, on the return scan, as the applied potential on working 
electrode potential becomes more negative than the reduction potential of a redox couple, a reduction process (i.e. gain of electrons by the solution from the electrode) occurs to cause a cathodic current. By IUPAC convention, anodic currents are positive and cathodic currents are negative.

The CV shown in Figure 2.11 starts at a slightly negative potential, (A) up to some positive switching value, (C) at which the scan is reversed back to the starting potential. As the potential increase to a value high enough to cause oxidation, an anodic current will be generated. As the potential continue to increase, the anodic current will increase and reach to the anodic current peak at $\mathrm{B}\left(\mathrm{I}_{\mathrm{pa}}\right)$ when all the reduced form of species at the electrode surface is consumed at corresponding potential $\mathrm{E}_{\mathrm{pa}}$. Due to oxidation, the concentration of the reduced species $\left(\mathrm{C}_{\mathrm{R}}\right)$ at the electrode surface decreases as compared to bulk of the solution. This will create a concentration gradient which causes more transport of reduced species towards the electrode by diffusion. The rate of this mass transport is governed by Fick's first law of diffusion and determined the magnitude of current. At point $\mathrm{C}$, the rate of mass transport reached to a maximum due to largest concentration gradient. When potential are further increased, current will decrease due to the decrease in the concentration gradient. The potential reaches the preset upper limit at $\mathrm{C}$, and reverses its direction. In the reverse direction reduction will occur the applied potential is sufficient and will reduce the oxidized species. When the applied potential gets closer to $\mathrm{E}_{\mathrm{pc}}$, ithe reduction process will be initiated and generate the cathodic current. As the potential 
decrease further, the cathodic current will increase to its peat at $\mathrm{D}\left(\mathrm{I}_{\mathrm{pc}}\right)$ as all the oxidized form of species at the electrode surface is consumed at corresponding potential $\mathrm{E}_{\mathrm{pc}}$. The potential scans back to the preset lower limit [10-11].

Given that a redox reaction is reversible, and that the redox products are stable, for such a system it can be shown that:

1. The corresponding peak potentials $\mathrm{E}_{\mathrm{pa}}$ and $\mathrm{E}_{\mathrm{pc}}$ are independent of scan rate and,

2. The formal potential for a reversible couple $\mathrm{E}^{0^{\prime}}$ is centered between $\mathrm{E}_{\mathrm{pa}}$ and $\mathrm{E}_{\mathrm{pc}}$ : $\mathrm{E}^{0^{\prime}}=\left(\mathrm{E}_{\mathrm{pa}}+\mathrm{E}_{\mathrm{pc}}\right) / 2$

\subsection{Amperometry}

The performance of biosensors can be characterized by the measurement of amperometric response. The amperometric response curves of our glucose biosensors are obtained by using a potentiostat (CHI 660C) in a three electrode setup similar to the $\mathrm{CV}$ measurement discussed above. In this measurement a constant potential is applied at the working electrode using the potentiostat. Dependence of current output response at the working electrode on time is measured. This potential must have a magnitude sufficient to initiate the related redox process. More details are discussed in chapter 4 . 


\subsection{Polarization measurements}

The performance of biofuel cells can be characterized by measuring the polarization curves. Polarization curves of glucose $/ \mathrm{O}_{2}$ biofuel cells are obtained using a galvanostat/ potentiostat (CHI 660C) in a two electrode setup. For two electrode measurements reference and counter electrodes are shorted. In this case, the potential difference between the two electrodes (biocathode and bioanode) is measured across a series of resistance, i.e., as a function of current. More details are discussed in chapter 5.

\subsection{Conclusions}

Brief introduction of various characterization techniques used was presented in this chapter. This includes SEM, TEM, Raman, FTIR, CV, Amperometry and polarization measurements.

\subsection{References}

1. J. I. Goldstein, C. E. Lyman, D. E. Newbury, E. Lifshin, P. Echlin, L. Sawyer, D. C. Joy, J. R. Michael. Scanning electron microscopy and X-Ray microanalysis, Third edition, Plenum Press (New York Boston, Dordrecht, London, Moscow), 2003.

2. H. Bethge, J. Heydenreich. Electron microscopy in solid state physics, Elsevier (Amsterdam, Oxford, New York) 1987. 
3. D. B. Williams, C. B. Carter. Transmission electron microscopy; Basics I, Plenum Press (New York, London), 1996.

4. H. W. Kroto, J. R. Heath, S. C. O’Brien, R. F. Curl, R. E. Smalley. C60 : Buckminsterfullerene, Nature, 1985, 318, 162.

5. M. A. Pimenta, A. Marucci, S. Empedocles, M Bawendi, E. B. Hanlon, A M Rao, P.C. Eklund, R. E. Smalley, G. Dresselhaus, M. S. Dresselhaus. Raman modes of metallic carbon nanotubes, Phys. Rev. B, 1998, 58, R16016.

6. S. D. M. Brown, A. Jorio, P. Corio, M. S. Dresselhaus, G. Dresselhaus, R. Saito, K. Kneipp. Origin of the Breit-Wigner-Fano lineshape of the tangential G-band feature of metallic carbon nanotubes, 2001 Phys. Rev. B63 155414.

7. B. Schrader. Infrared and raman spectroscopy, VCH (Weinheim, New York, Basel, Cambridge, Tokyo), 1995.

8. J. R. Ferraro, K. Nakamoto, Introductory raman spectroscopy, Academic Press (Boston, San Diego, New York, London, Sydney, Tokyo, Toronto) 1994.

9. D. L. Pavia, G. M. Lampman, G. S. Kriz, Introduction to spectroscopy, Harcourt Brace College Publishers, (Fort Worth, Philadelphia, San Diego, New York, Austin, London, Sydney, Tokyo) 1996.

10. A.J. Bard, L.R. Faulkner, Electrochemical methods: Fundamentals and applications (2 ed. Wiley. ISBN 0471043729) New York 568

11. Electrochemistry of carbon nanotubes, Ladislav Kavan and Lothar Dunsch, 2008, Volume 111/2008, 567-603, 


\section{Chapter 3}

\section{Enhanced Field emission from CNTs}

\subsection{Introduction}

CNTs are promising materials for electron field emission due to their small tip radius, high aspect ratio, and robust mechanical and chemical properties [1-4]. Although field emissions from CNTs are known for more than a decade [5], reliable commercial products are yet to be realized. Obviously, the basic science for stable field emission with high emission density is still not clear. Most reported work focus on demonstrating low emission threshold fields $\left(E_{t h}\right)$ of various types of CNTs [6-9] and their device configuration [10]. In this work we tried to improve the issues involved in field emission of as grown CNTs.

\subsubsection{Field emission theory}

Electron field emission is the extraction of electrons from the metal surface under the application of very high external electric field. It involves the tunneling through the surface potential barrier. Figure 3.1 shows a schematic diagram of tunneling process in metals under the influence of high electric field. When there is no external applied electric field, the potential barrier is rectangular. When the negative potential is applied to the metal surface it becomes triangular. The slope of later depends on the amount of the local electric field just above the surface. This local electric field is radically enhanced when the emitter tip is very sharp and has high aspect ratios especially in the case of CNTs [11]. 


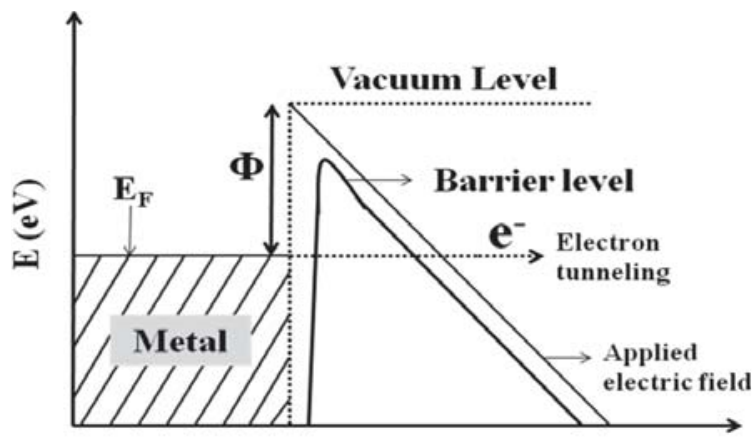

Distance ( $\mathbf{A})$

Figure 3.1: Potential energy diagram [11]

The emission current from a metal surface is determined by the FowlerNordheim (F-N) equation which is:

$J=A \beta^{2} E^{2} \exp \left(-B \Phi^{3 / 2} / \beta E\right)$ and is often use to describe field emission, where $A, B$ are constants, $E$ is the applied electric field in $\mathrm{V} \mathrm{cm}^{-1}$, and $\Phi$ is the work function in $\mathrm{eV}, \beta$ is the field enhancement factor.

The F-N equation gives the relationship between the emission current density J, the electric field $\mathrm{E}$ and the work function $\Phi$ and is based on following assumptions:

- Free electron approximation applies inside the metals only.

- Metal temperature is $0^{0} \mathrm{~K}$.

- No irregularities should be present at the metal surface.

Smooth surface of metals, with a given value of work function have extremely high threshold electric field $\left(\sim 10^{4} \mathrm{~V} / \mu \mathrm{m}\right)$. Now, work function of a material cannot be altered significantly. Hence, field emission sources rely on sharp tips instead of flat 
surfaces. Local electric field enhancement at an ultra-sharp sharp tip is extremely high due to its sharp curvature. This is the reason for high electric field field enhancement factor " $\beta$ " for sharp tips. larger value of $\beta$ leads to lower effective threshold voltage for field emission.

\subsection{Current state of research in field emission from CNTs}

Numerous techniques have been studied to enhance the performance of multiwalled carbon nanotubes (MWCNTs) for electron field emission [12-14]. Many reported that after etching the tips of the CNTs, the field emission properties enhanced [15-16]. However there is controversy among those reported literatures [17-18]. Various research groups also attempted to modify the properties of CNTs by coating of conductive oxide $\left(\operatorname{In}_{2} \mathrm{O}_{3}\right)$, wide band gap materials, polymer, $\mathrm{MgO}$ and Barium Strontium oxide [19-23]. Most of these works focused on reducing the threshold electric field for field emission. However, device lifetime, long-term emission stability, and emission density are scarcely discussed. Poor device lifetime due to resistive heating and mechanical failure at the interface of CNTs/substrate has been reported [24]. Recently, we started to investigate factors that determine the emission stability of CNTs and found that the graphitic order of CNTs is one of the key factors for stable emission [25]. Our recent works also evaluate the fundamental factors behind stable field emission and high emission density. In this chapter, we found that both screening effects and catalyst removal are important for improving the emission stability and density of VA-MWCNTs. Although screening effect was known to 
determine the emission density, its contribution to emission stability is not clear [26]. However field emission from CNTs suffers from high emission threshold voltage, poor emission density and poor long term emission stability. To enhance field emission from CNTs, I fabricated three types of devices using VA-MWCNTs: a) Etched and bundled MWCNTs, b) Opened tip MWCNTs embedded in PMMA matrix

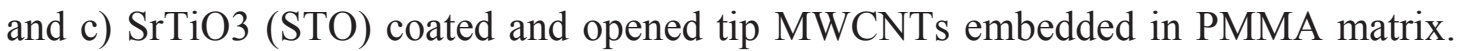
These new assemblies now open the door for commercial and practical application of CNTs in field emission display devices.

\subsection{Experimental methods to enhance the field emission properties from as grown CNTs}

In this section experimental procedure of different methods to enhance the field emission from as grown CNTs is discussed.

\subsubsection{Fabrication of etched and conical self-assembled bundles of CNTs}

The samples were prepared by dual radio frequency PECVD techniques [27]. In brief, Ni films (10 $\mathrm{nm}$ thick) were first deposited on p-type Si substrates $(1-10 \Omega \mathrm{cm})$ by RF

magnetron sputtering. These substrates were then used for the growth of VAMWCNTs at $450^{\circ} \mathrm{C}$ by using pure methane gas. Our VA-MWCNTs were grown within a circular area $(7 \mathrm{~mm}$ in diameter). The morphology of the as grown CNTs sample can be seen in Figure 3.2 (a) and (b). PECVD growth produces CNTs with Ni catalyst particle on top of carbon nanotubes as can be seen in Figure 3.2 (b). The length of these CNTs is of the order of few micrometers $(3-4 \mu \mathrm{m})$ and diameter of 
these CNTs is of the order if few nanometers (40-60nm). These CNTs are defective because of plasma used in the growth process.
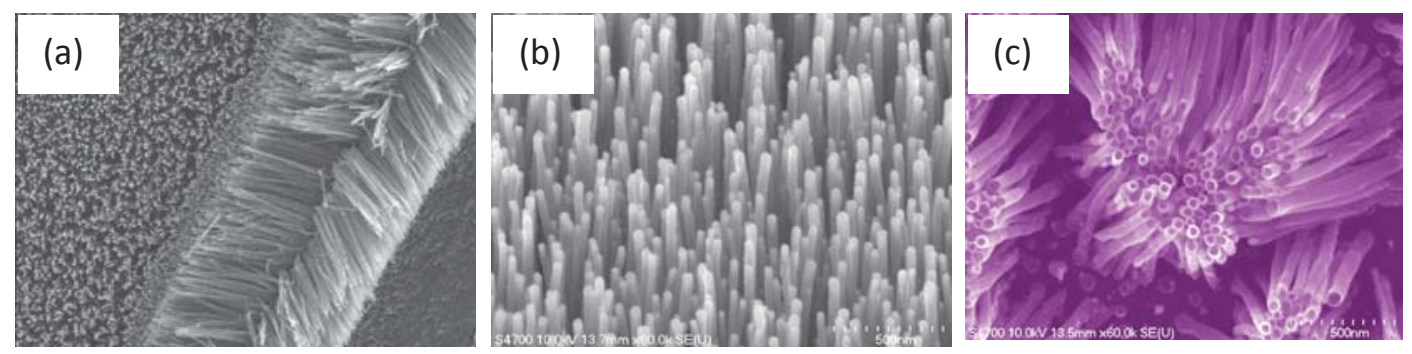

Figure 3.2: SEM image of as grown CNTs (a) low magnification (b) high magnification and (c) Etched and Bundled CNTs

Three identical samples were prepared in each growth process. The residual Ni catalytic nanoparticles in the samples can be removed from the tips of VA-MWCNTs by etching in nitric acid $\left(\mathrm{HNO}_{3}\right)(70$ vol. $\%$, for $\sim 5 \mathrm{~min})$. The etched samples were then rinsed with de-ionized water and toluene. These processes lead to catalyst-free MWCNTs. Figure 3.2 (c) shows the morphology after etching and bundling of CNTs.

\subsubsection{Fabrication of PMMA-CNTs matrices}

In this method we have created PMMA-CNT matrices by embedding openedtip VA-MWCNTs with PMMA. The details are described below. The fabrication scheme of the PMMA-CNT matrices using PMMA solution is summarized in Figure 3.3. The PMMA solution was prepared by diluting PMMA with the developer solution (Microchem) in a volume ratio of 1:1. As-grown VA-MWCNTs samples (Figure 3.3 
(a)) were dipped into the solution for 15 minute and followed by an annealing process at $\sim 100^{\circ} \mathrm{C}$ for $2-3$ minutes.
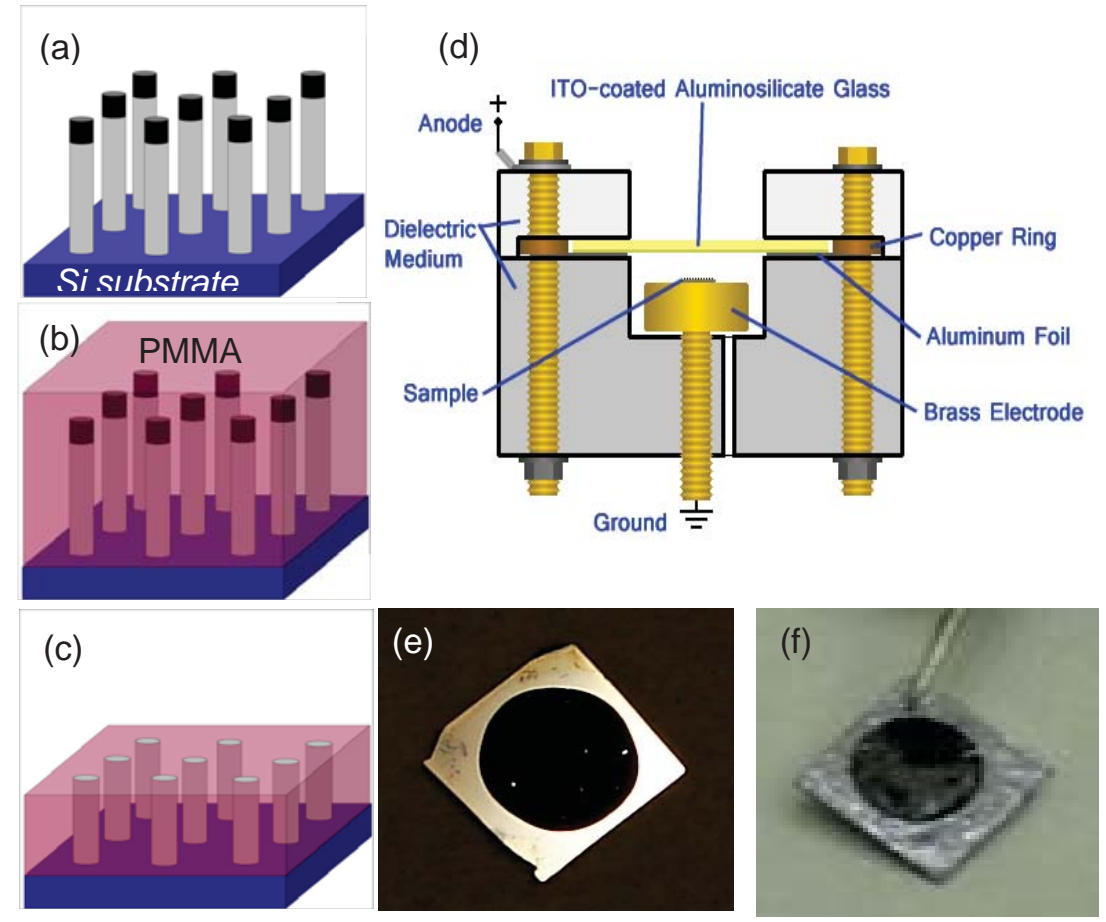

Figure 3.3: Schematic for the fabrication of PMMA-CNT matrices. (a) As-grown VAMWCNTs on a Si substrate with Ni nanoparticles at their tips. (b) VA-MWCNTs embedded with PMMA. (c) A PMMA-CNT matrix with opened-tip VA-MWCNTs after mechanical polishing. (d) Schematic of the field emission set-up. Appearances of the (e) as-grown sample and the (f) PMMA-CNT matrix. Reprinted with permission from [28]

The cured samples (Figure 3.3 (b)) were then mechanically polished by using fiber-free lapping cloth and a colloidal silica $(0.02 \mu \mathrm{m}$ in diameter $)$ solution to expose the tips of the CNTs. The PMMA-CNT matrices with opened-tip CNTs will finally be formed (Figure 3.3 (c)) and ready for the field emission measurements. 
Figure 3.3 (d) shows the schematic of our field emission set-up with indium thin oxide (ITO) coated on alumina-silicate glasses as the anode. For the characterization of electron field emission, all samples were attached on the brass cathode in a planar diode configuration by applying a thin layer of silver paste. The spacing between the ITO anode and the tips of the CNTs was maintained at $1000 \pm 10$ $\mu \mathrm{m}$. Electron emission was tested at a base vacuum pressure of $\sim 1.5 \times 10^{-8}$ Torr. The appearance of the as-grown sample and the PMMA-CNT matrices are shown in Figure 3.3 (e) and (f) respectively.

\subsubsection{Fabrication of PMMA-SrTiO ${ }_{3}$-CNTs matrices}

In this section the as grown CNTs was coated with $\mathrm{SrTiO}_{3}$ (STO) and PMMA. The fabrication scheme of the PMMA-STO-CNTs matrices is summarized in Figure 3.4. The STO-CNT samples were prepared by coating $\mathrm{SrTiO}_{3}(\sim 20 \mathrm{~nm}$ thick) on asgrown VA-MWCNTs by pulsed-laser deposition at room temperature.

The preparation of PMMA-STO-CNT matrices was carried out by dip coating $\mathrm{SrTiO}_{3}$ coated VA-MWCNTs by PMMA and then followed by mechanical polishing. The PMMA solution was prepared by diluting PMMA with the developer liquid (Microchem) in a volume ratio of 1:1.

The STO-CNT samples were dipped into the solution for 15 minute and followed by annealing $\left(\sim 100^{\circ} \mathrm{C}\right.$ for $2-3$ minutes). The cured samples were then mechanically polished by using fiber-free lapping cloth and a colloidal silica $(0.02 \mu \mathrm{m}$ 
in diameter) solution to expose the tips of the CNTs. The appearance of the as-grown sample, STO coated CNTs sample and the PMMA-STO-CNT matrix are shown in Figure $3.4(\mathrm{e})-(\mathrm{g})$ respectively.
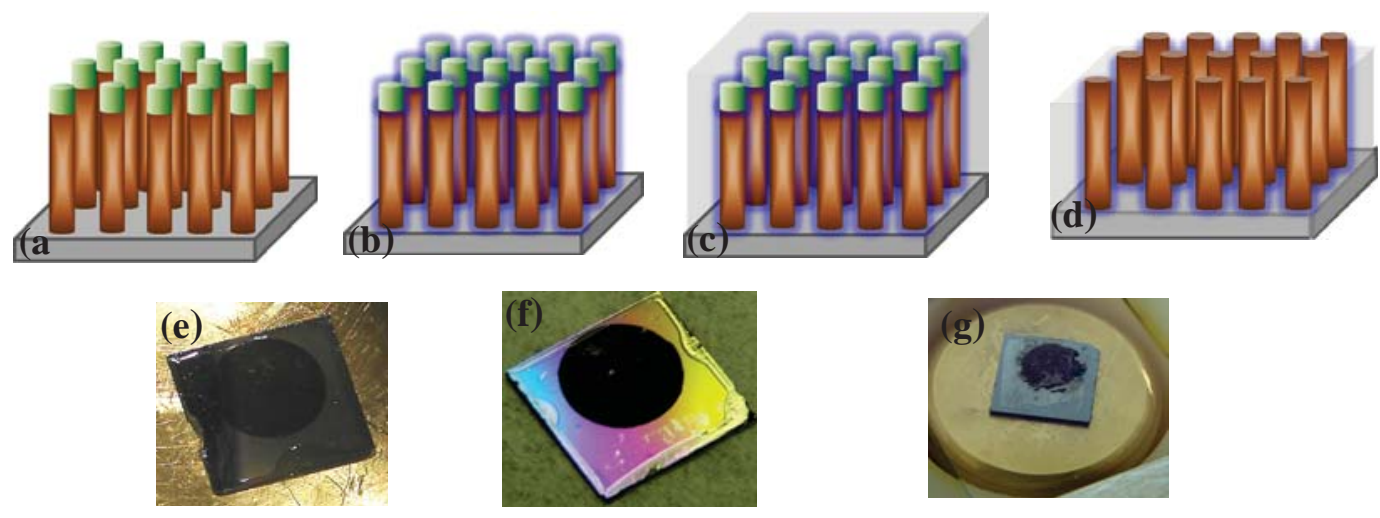

Figure 3.4: Schematic for the fabrication of PMMA-STO-CNT matrices. (a) As-grown VAMWCNTS on a Si substrate with Ni nanoparticles at their tips. (b) VA-MWCNTs coated with $\mathrm{SrTiO}_{3}$. (c) STO coated VA-MWCNTS embedded with PMMA. (d) A PMMA-STO-CNT matrix with opened-tip VA-MWCNTs after mechanical polishing. Appearances of the (e) as-grown sample, (f) STO coated sample, (g) PMMA-STO-CNT matrix after polishing.

\subsection{Results and discussion}

\subsubsection{Field emission from etched and conical self-assembled bundles of CNTs}

All of our samples were characterized by field emission scanning electron microscopy (FESEM) and Raman spectroscopy (laser wavelength $\sim 632 \mathrm{~nm}$, laser spot size $\sim 1 \mu \mathrm{m}$ in diameter). The field emission measurements were conducted in a planar diode configuration at a base vacuum pressure of $10^{-7} \mathrm{mbar}$ [13]. The spacing between the anode (Indium Tin Oxide/ITO film on glass) and the tips of the VA-MWCNTs was maintained at $1000 \pm 10 \mu \mathrm{m}$ without using dielectric spacer. 
All measurements were re-confirmed by repeating measurement on the same sample as well as another set of sample prepared in the same growth process and treatment. As shown in Figure 3.5 (a) and (b), we found that rinsing of toluene after acid etching can maintain the vertical alignment of the opened-tips MWCNTs. Tentatively we think that this is due to the lower surface tension of toluene than water ( 0.0287 versus 0.0735$)$.
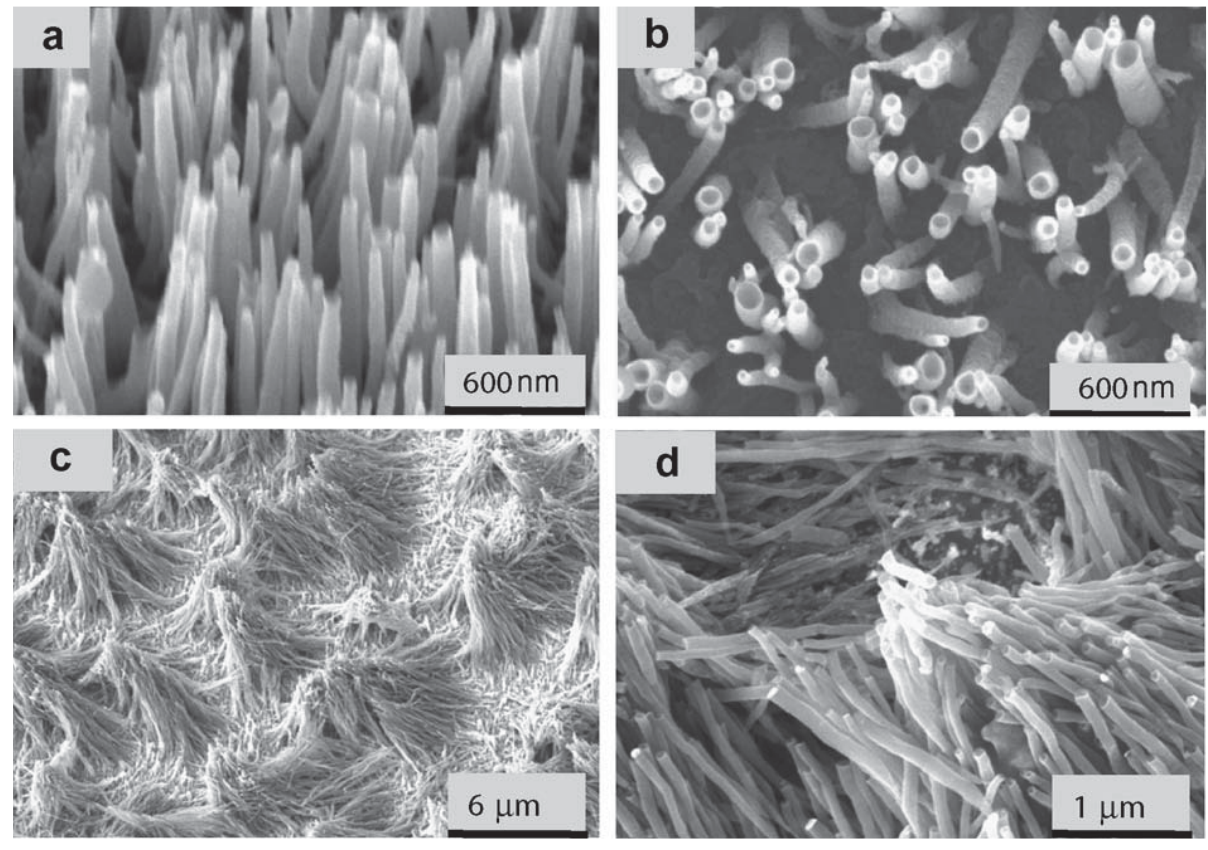

Figure 3.5: Typical appearance of opened-tip VA-MWCNTs ( $a$ and b) and arrays of opened tip, conical bundles and low (c) and high (d) magnification. Reprinted with permission from [16]

Lower surface tension will reduce the van der Waals forces between CNTs and toluene that will pull away CNTs to each other during the drying process. However, toluene alone is insufficient to maintain the vertical alignment of VA-MWCNTs that 
are smaller in diameters. As shown in Figure 3.5 (c) and (d), VA-MWCNTs with diameters $<60 \mathrm{~nm}$ will self assembled into conical bundles after etching. Obviously, the function of toluene (versus water) on maintaining the vertical alignment of these VA-MWCNTs would be an interesting topic for future investigation. We have first compared as grown MWCNTs with the opened-tip, catalyst-free VA-MWCNTs.
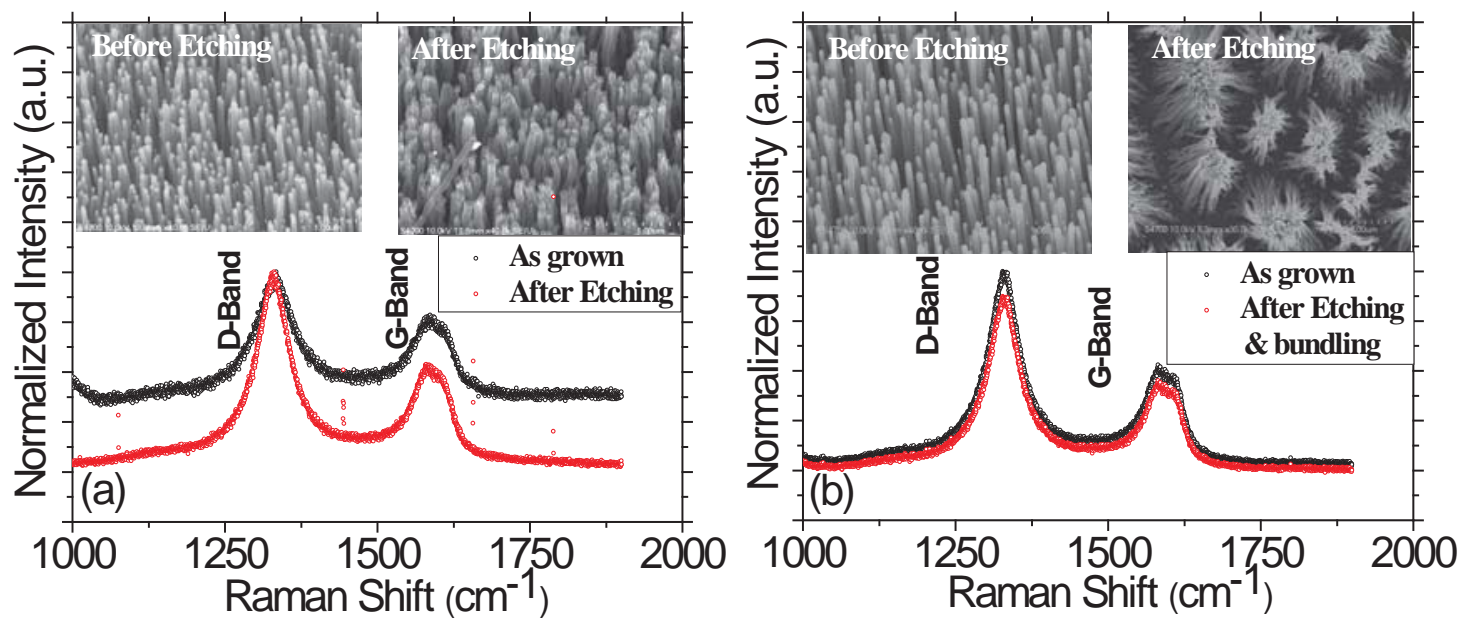

Figure 3.6: (a) Raman spectra for the first set of samples. The as grown and the etched VAMWCNTs are shown in the insets. (b) Raman spectra for the second set of samples. The as grow and the etched and bundled samples are shown in the insets. Reprinted with permission from [16]

The SEM image of the as grown sample (left inset) and their Raman spectra are shown in Figure 3.6 (a). These VA-MWCNTs were $\sim 4 \mu \mathrm{m}$ in length and $\sim 80 \mathrm{~nm}$ in diameter. The graphitic order of these MWCNTs was examined by comparing the intensity of the graphitic (G) and defective (D) Raman bands. The G and D bands 
represent the zone center phonons of $E_{2 g}$ symmetry and the K-point phonons of $A_{1 g}$ symmetry, respectively [14]. The intensity ratio $\left(\mathrm{I}_{\mathrm{G}} / \mathrm{I}_{\mathrm{D}}\right)$ for the as grown and the etched VA-MWCNTs are $\sim 0.88$ and $\sim 0.78$, respectively as shown in Figure 3.6 (a). The difference is within the measurement deviation within a sample.

For the second set of samples, the $\mathrm{I}_{\mathrm{G}} / \mathrm{I}_{\mathrm{D}}$ ratios for the as grown and the etched and bundled samples remain at $\sim 0.75$ as shown in Figure 3.6 (b). From these results, we conclude that etching will not change the graphitic order of MWCNTs since carbon are inert to acids.

Figure 3.7 (a) shows the current density (J) versus electric field (E) characteristics for as grown VA-MWCNTs sample. The Fowler-Nordheim (F-N) equation [15], $J=A \beta^{2} E^{2} \exp \left(-B \Phi^{3 / 2} / \beta E\right)$ is often use to describe field emission, where A, B are constants, $E$ is the applied electric field in $\mathrm{Vcm}^{-1}$, and $\Phi$ is the work function in $\mathrm{eV}, \beta$ is the field enhancement factor. A linear F-N plot (inset of Figure 3.7 (a)) verified that the detected currents are due to quantum tunneling. The threshold electric field, $E_{t h}$ (applied electric field for generating a current density of $1 \mu \mathrm{A} / \mathrm{cm}^{2}$ ) is 3.10 $\mathrm{V} / \mu \mathrm{m}$ for the as grown sample. The J-E and corresponding F-N plots for the etched VA-MWCNTs are also shown in Figure 3.7 (a). This etched sample has identical $E_{t h}$ and showing linear F-N relation. The emission stability of these two samples was then compared. As shown in Figure 3.7 (b), the etched VA-MWCNTs seem to have smaller degradation in current density after $\sim 1200$ min of emission test. 
Apparently, the removal of residual catalyst particles from the tips of VAMWCNTs does not reduce $E_{t h}$ but can improve the long-term emission stability.
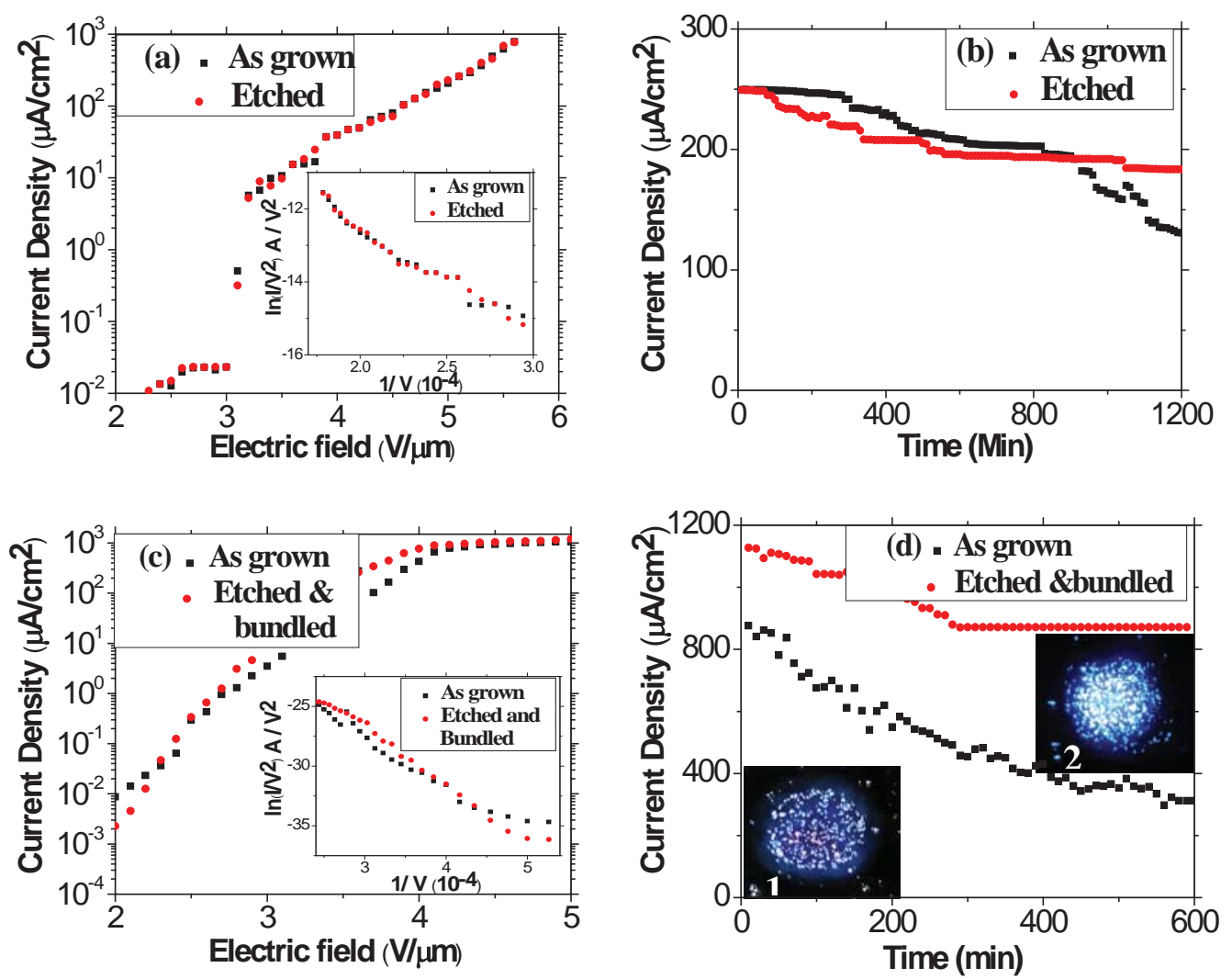

Figure 3.7: (a) The field emission characteristic curves for the as grown and the etched VAMWCNTs shown in Figure 3.5(a). The related Fowler-Nordheim (FN) plots are shown in the insets. (b) The related emission current stability curves. (c) The field emission characteristic curves for the as grown and the etched and bundled MWCNTs shown in the insets of Figure 5(b). The related Fowler-Nordheim (FN) plots are shown in the insets. (d) The related emission current stability curves. Insets in (d) show the florescence on the ITO electrode as induced by the emitted electrons fromthe as grown (Inset 1) and the etched and bundled (Inset 2) CNTs. Reprinted with permission from [16]

We think that residual metallic catalytic particles that have lower melting point than CNTs $\left(\sim 1452^{\circ} \mathrm{C}\right.$ for bulk nickel versus $>3650^{\circ} \mathrm{C}$ for graphite $)$ may create some 
unknown effects on the emission stability when significant Joule heating was introduced during the prolong emission stability test. The actual mechanism is not clear at present and is subjected for future investigation.

We have tested the second set of samples (tube diameter $\sim 60 \mathrm{~nm}$ ) to understand the effect of bundling. As shown in Figure 3.7 (c), $E_{t h} \sim 2.60 \mathrm{~V} / \mu \mathrm{m}$ are detected from both the as grown and the etched and bundled samples. The linear F-N relations (inset of Figure 3.7 (c)) were also revealed. Current saturation at high applied fields is detected in these samples. We think that electron supply is limited by the impedance (mostly resistance, but may include some capacitance and inductance) present especially along the CNTs and at the contacts between the CNTs and the substrate. These limiting factors become obvious at high current density probably due to Joule heating and/or current-induced dislocation [11]. As shown in inset of Figure 3.6 (a) the distances between the as grown VA-MWCNTs are small ( $~ 50-300 \mathrm{~nm})$ and will initiate screening effect that reduced the $\beta$ factors. This means not all the as grown VAMWCNTs will contribute to the collected current except those are longer in lengths or located at the edges of the larger spacing. For the etched and bundled sample shown in Figure 3.5 (c), the distances between bundles are more than one micrometer. Thus, each bundle can be considered as a larger emission pyramid. We have compared these samples for their emission stability. As shown in Figure 3.7 (d), the etched and bundled sample is stabilized at a current density $>800 \mu \mathrm{A} / \mathrm{cm}^{2}$ after continuous 20-h operation, while the as grown sample has reduced its current density 
to $<400 \mu \mathrm{A} / \mathrm{cm}^{2}$. As shown in the insets of Figure 3.7 (d), the emission density for the etched and bundled sample (inset 2) is higher than that of the as grown sample (inset 1). Apparently, lower screening effects on the bundled sample offers more emission sites. Since the emission loads (heat and mechanical stress from Joule heating) is now shared by more CNTs, the emission stability is thus improved. Theoretical simulation (to be discussed hereafter) suggests that lower local electric field is applied on these bundles. As indicated by the F-N equation, a lower local field on each emitter will lead to the emission of lower current density per emitter. This will reduced Joule heating and stresses on these emitters and thus produce stable emission. It is interesting to see that both the as grown and bundled samples are by chance having identical $E_{t h}$. This is explained as follows. The measured emission current from a sample is actually depends on both the current emitted from each emitters and the emitter density. Thus $E_{t h}$ is also depends on these factors. As our bundled sample has an $E_{t h}$ identical to that of the as grown sample, its higher emitter density suggests that the current emitted from each emitter in the bundled sample should be lower than that in the as grown sample. This interpretation is consistent to the results generated from our simulation to be described hereafter.

To further support our discussion, we have performed simulation by using the COMSOL MULTIPHYSICS software (Parameters: diameter of CNTs, DCNTs $=40$ $\mathrm{nm}$; Length of CNTs, LCNTs $=4 \mu \mathrm{m}$; edge to edge spacing between CNTs, $\mathrm{S}=40$ $\mathrm{nm}$; applied electric field between top and bottom boundaries, $\left.E_{a p p l}=5 \mathrm{~V} / \mu \mathrm{m}\right)$. 

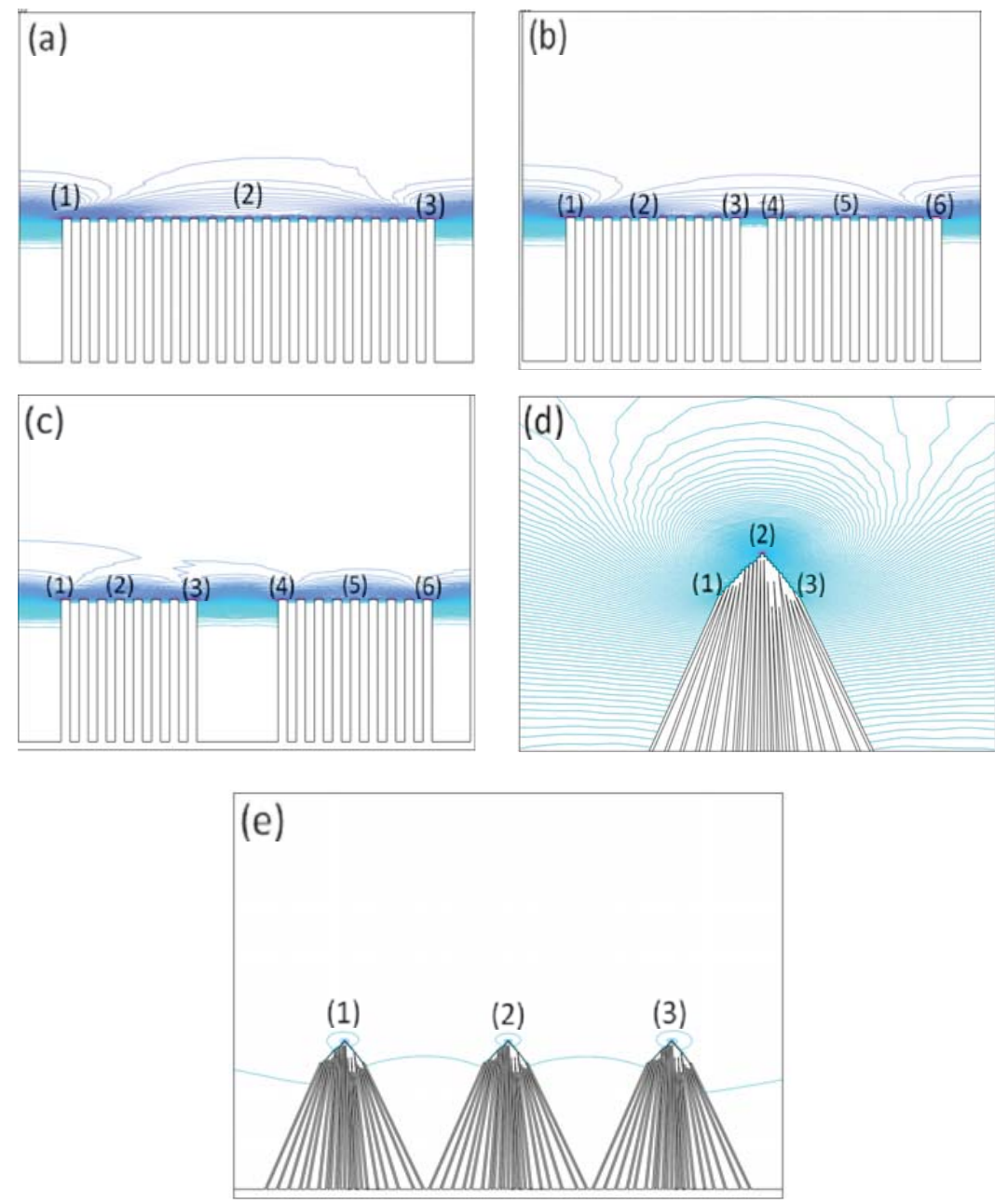

Figure 3.8: Schematic of the simulated potential maps for (a) an array of VA-MWCNTs, (b) two arrays of VA-MWCNTs with a $120 \mathrm{~nm}$ spacing in between, (c) two arrays of VA-MWCNTs with a $280 \mathrm{~nm}$ spacing in between, (d) a conical bundle, and (e) three conical bundles. Reprinted with permission from [16]

Simulation for an array of VA-MWCNTs (Figure 3.8 (a)) shows that CNT at the center has lowest local electric field due to the screening effects from the surrounding CNTs $\left(6.713 \times 10^{6} \mathrm{~V} / \mathrm{m}\right.$ at point 2 versus $\sim 1.035 \times 10^{7} \mathrm{~V} / \mathrm{m}$ and $\sim 1.038 \mathrm{x}$ 
$10^{7} \mathrm{~V} / \mathrm{m}$ at points 1 and 3, respectively). We further simulate the effect of the gap (S) between two small arrays of VA-MWCNTs.

Figure 3.8 (b) shows two arrays of VA-MWCNTs with $\mathrm{S}=120 \mathrm{~nm}$. We observe that the local fields at both sides of the gap (point 3: $\sim 9.15 \times 106 \mathrm{~V} / \mathrm{m}$, and point 4: $\sim 8.91 \times 106 \mathrm{~V} / \mathrm{m}$ ) are higher than those at the centers of the two arrays (points 2: $\sim 5.55 \times 106 \mathrm{~V} / \mathrm{m}$, and point 5: 5.48 $\times 106 \mathrm{~V} / \mathrm{m}$ ). Highest field is still observed at points 1 and $6(\sim 1.037 \times 107 \mathrm{~V} / \mathrm{m}$ and $\sim 1.027 \times 107 \mathrm{~V} / \mathrm{m})$, i.e., edges close to the boundaries where no CNTs (and no screening effect) is found at one side. We have compared this to the case with $\mathrm{S}=360 \mathrm{~nm}$. We observe that the local fields at points 3 and $4\left(\sim 1.041 \times 10^{7} \mathrm{~V} / \mathrm{m}\right.$ and $\left.\sim 1.064 \times 10^{7} \mathrm{~V} / \mathrm{m}\right)$ is higher than those at points 2 and 5 $\left(\sim 6.30 \times 10^{6} \mathrm{~V} / \mathrm{m}\right.$ and $\left.\sim 7.65 \times 10^{6} \mathrm{~V} / \mathrm{m}\right)$ and comparable to those at points 1 and 6 $\left(\sim 1.022 \times 10^{7} \mathrm{~V} / \mathrm{m}\right.$ and $\left.\sim 1.068 \times 10^{7} \mathrm{~V} / \mathrm{m}\right)$. We thus conclude that screening effect has reduced as the gap between CNT arrays increased to $360 \mathrm{~nm}$.

We have simulated the local electric field for one conical bundle of CNTs as shown in Figure 3.8 (d). The local field at point $2\left(\sim 5.9743 \times 10^{4} \mathrm{~V} / \mathrm{m}\right)$ is more than those at points 1 and $3\left(\sim 4.058 \times 10^{4} \mathrm{~V} / \mathrm{m}\right.$ and $\left.\sim 3.712 \times 10^{4} \mathrm{~V} / \mathrm{m}\right)$. This means, emission is more likely from CNTs located near the center of the bundles. Also, these values are two-order of magnitude lower than those discussed earlier for CNT arrays. As suggested by the F-N equation, lower local fields on the bundles means lower current density will be emitted from each bundle, as consistent to our earlier interpretation. This means, heat and mechanical stresses introduced on CNTs due to 
Joule heating are lower for the case of nanotube bundles. Finally, we have simulated electric field applied on an array of CNT bundles. As shown in Figure 3.8 (e), electric fields are $\sim 6.2182 \times 10^{4} \mathrm{~V} / \mathrm{m}, \sim 5.2582 \times 10^{4} \mathrm{~V} / \mathrm{m}$, and $\sim 6.789 \times 10^{4} \mathrm{~V} / \mathrm{m}$ at bundle 1,2 and 3 , respectively. Since the local field at bundle 2 is approaching that simulated in Figure 3.8 (d) (which has minimum screening effect), this means, the investigated gap ( $\mathrm{S}=2 \mu \mathrm{m}$ ) between bundles is sufficient to reduce the screening effects and enabled emission from most bundles. This is consistent with the higher emission density shown in Figure 3.8 (d). As the collected current is contributed by more emission sites, the current loading on individual bundles is lower as compared to the loading on individual CNTs in the case of CNT arrays.

\subsubsection{Field emission from PMMA-CNTs matrices}

Figure 3.9 shows the scanning electron microscope (SEM) images and the related raman spectroscopy for all the samples. All these samples are having VAMWCNTs of $\sim 4 \mu \mathrm{m}$ in length and $\sim 40 \mathrm{~nm}$ in diameter (Figure 3.9 (a)).

Figure 3.9 (b) shows that the as-grown VA-MWCNTs can be fully embedded in PMMA after the dip coating, and curing processes. Opened-tip VA-MWCNTs are exposed on the top surface of the PMMA-CNT matrix after polishing (Figure 3.9 (c)). The corresponding Raman spectra for the as-grown sample, the PMMA embedded sample, and the PMMA-CNT matrix, are shown in Figure 3.9 panels (d)-(f) respectively. These Raman spectra were obtained by a confocal Raman microscope using a laser excitation wavelength of $632 \mathrm{~nm}$. 

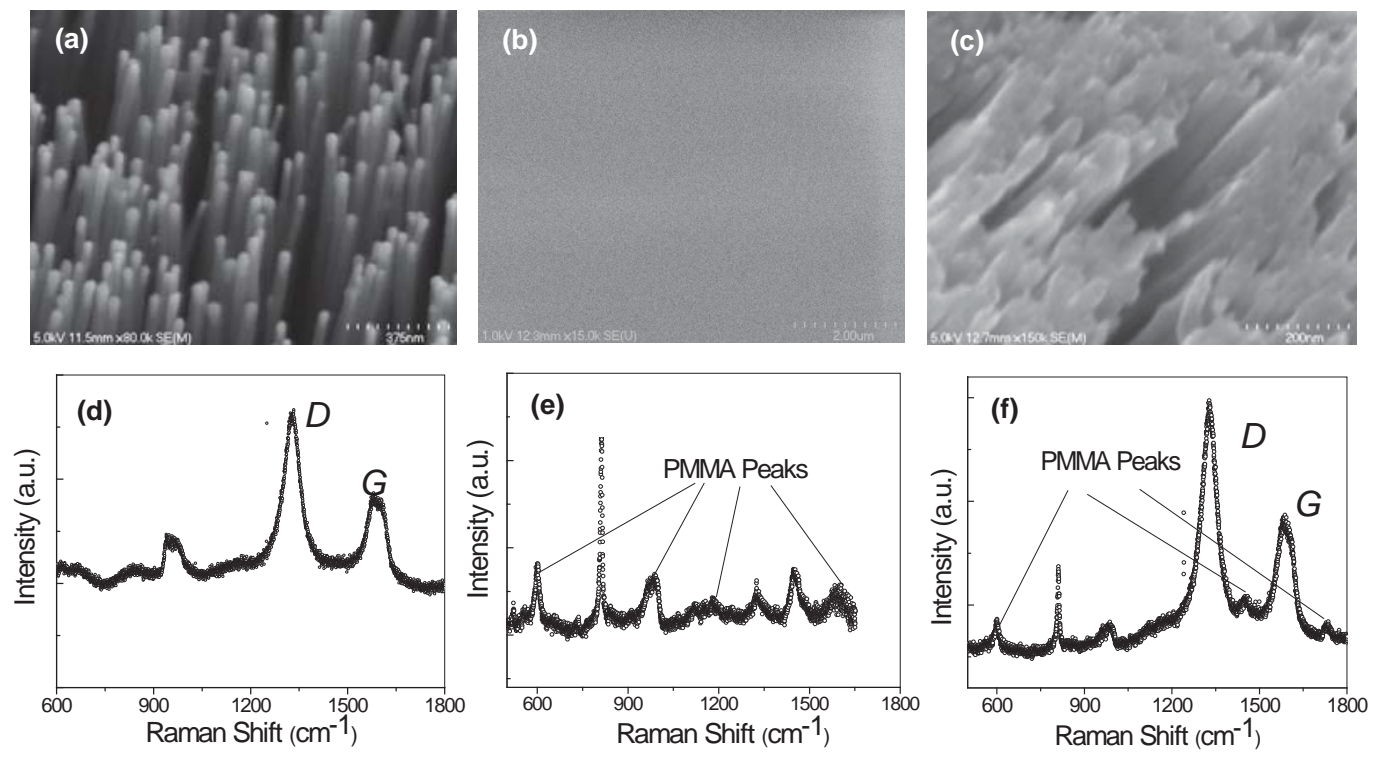

Figure 3.9: SEM images of (a) the as-grown sample, (b) ample embedded in PMMA, and (c) PMMA-CNT matrix with exposed CNT tips. (d), (e) and (f) are the corresponding Raman spectra. Reprinted with permission from [28]

The graphitic, $G\left(1580 \mathrm{~cm}^{-1}\right)$ and the disorder, $D\left(1330 \mathrm{~cm}^{-1}\right)$ peaks for VAMWCNTs in the as-grown sample are shown in Figure 3.9 (d). These peaks are diminishes in the PMMA embedded sample and only the PMMA Raman peaks are detected (Figure 3.9 (e)) [16]. The $G$ and $D$ peaks reappeared in the PMMA-CNT matrix as evident in Figure 3.9 (f).

Figure 3.10 (a) and (b) shows the emission current density $(J)$ versus the applied electric field $(E)$ for the as-grown sample and the PMMA-CNT matrix. The threshold electric field, $E_{t h}$, for the as-grown sample was found to be $3.898 \mathrm{~V} / \mu \mathrm{m}$ 

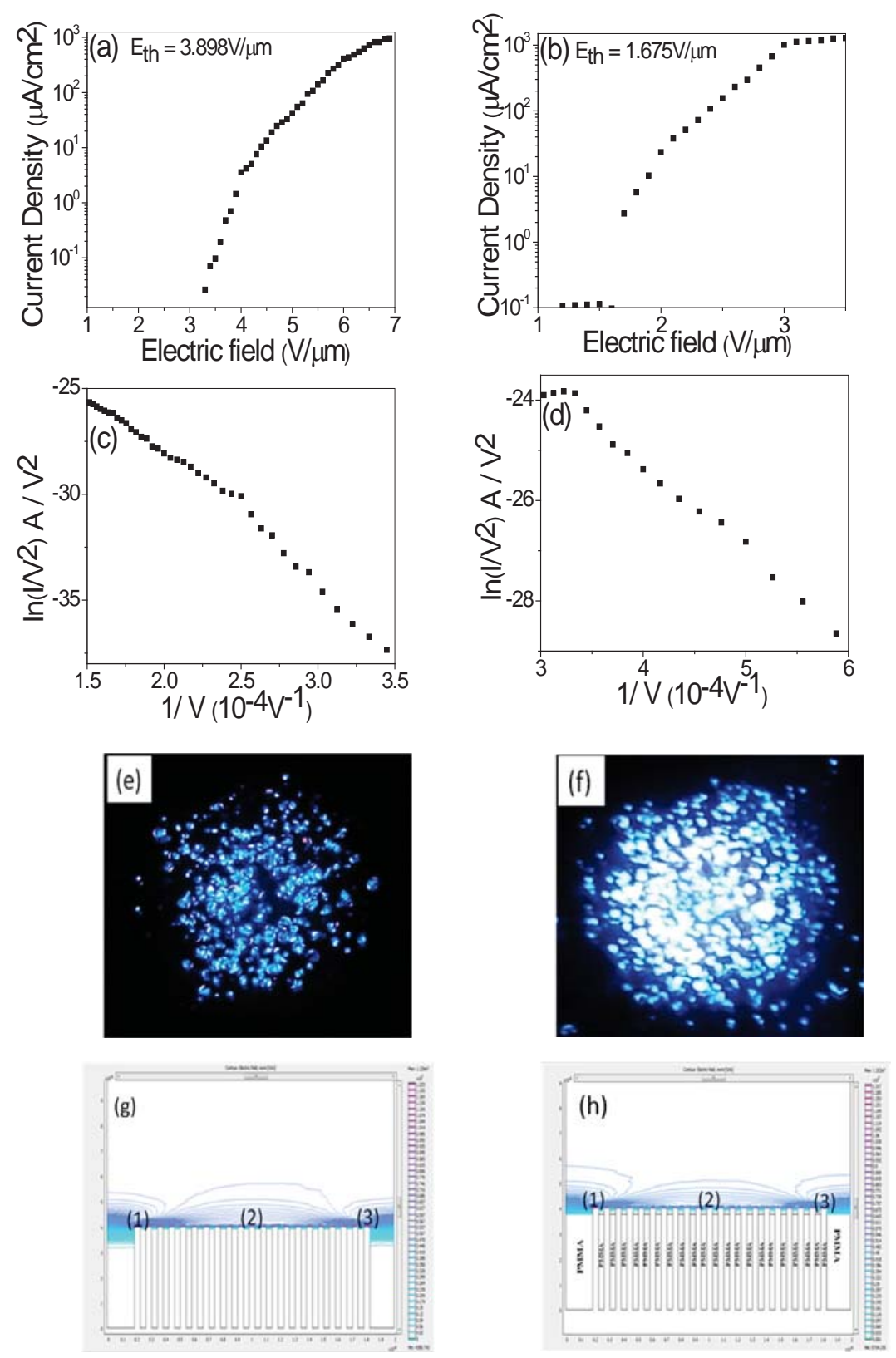

Figure 3.10: The emission current density (J) as a function of the applied electric field (E) for (a) the as-grown sample and (b) the PMMA-CNT matrix. The corresponding F-N plots for (c) as-grown sample and (d) the PMMA-CNT matrix. Image of the emission sites for (e) as-grown sample and (f) the PMMA-CNT matrix. The simulated potential maps for (g) the as-grown sample, and (h) the PMMA-CNT matrix with exposed CNT tips. Reprinted with permission from [28] 
(Figure 3.10 (a)). This threshold was reduced to $1.675 \mathrm{~V} / \mu \mathrm{m}$ for the PMMA-CNT matrix (Figure $3.10(\mathrm{~b})$ ).

The corresponding Fowler-Nordheim $(F-N)$ plots for these samples can be seen in Figure 3.10 (c) and (d). The linearity of these $F-N$ plots confirms the phenomena of field emission. The emission site densities as collected from the fluorescence on the ITO electrode are shown in Figure 3.10 panels (e)-(f) for the as-grown and the PMMA-CNT matrix, respectively.

As shown, the emission site density is much higher for the PMMA-CNT matrix despite the fact that it was collected from the ITO anode $1 \mathrm{~cm}$ above the CNT tips. The actual density would appear to be higher if the electrode gap is smaller since these electron beams are diverging from the VA-MWCNT tips. The circular shapes of the emission sites show that the electron field emission was occurring only from the areas where VA-MWCNTs were grown.

We attempt to understand the reduced $E_{t h}$ and enhance emission density from the PMMA-CNT matrix. Both $E_{t h}$ and emission density depends on the aspect ratio of the CNTs and their inter-tube spacing (screening effects). Since both the as-grown sample and the PMMA-CNT matrix are having VA-MWCNTs with identical aspect ratios and inter-tube spacing, the lower $E_{t h}$ and the enhance emission density detected from the PMMA-CNT matrix are related to the PMMA filling. A possible explanation is that the dielectric properties of PMMA have helped to reduce the screening effect between the CNTs. In order to verify this argument, we have simulated the screening effect of CNTs using the COMSOL Multiphysics software. The simulation parameters 
are as follows: diameter of CNTs, $D_{C N T s}=40 \mathrm{~nm}$; Length of CNTs, $L_{C N T s}=4 \mu \mathrm{m}$; edge to edge spacing between CNTs, $S=40 \mathrm{~nm}$; applied electric field between top and bottom boundaries, $\left.E_{a p p l}=5 \mathrm{~V} / \mu \mathrm{m}\right)$. Simulation for an array of as-grown VAMWCNTs (Figure 3.9g) shows that CNT at the center has lowest local electric field due to the screening effects from the neighboring CNTs $\left(\sim 9.89 \times 10^{6} \mathrm{~V} / \mathrm{m}\right.$ at point 1 , $\sim 6.21 \times 10^{6} \mathrm{~V} / \mathrm{m}$ at point 2 and $\sim 9.42 \times 10^{6} \mathrm{~V} / \mathrm{m}$ at point 3 , respectively). Next, we simulate for the case of PMMA-CNT matrix. The spaces between VA-MWCNTs are now simulated to have a dielectric constant, $\boldsymbol{k}=3.4$ [17]. As shown in Figure $3.10(\mathrm{~h})$, the local field at the centre of the PMMA-CNT matrix is $\sim 45 \%$ higher as compared with those of as grown VA-MWCNTs (point 2: $\sim 7.76 \times 10^{6} \mathrm{~V} / \mathrm{m}$ ). The local fields at the edges of the matrix (point 1: $\sim 1.04 \times 10^{7} \mathrm{~V} / \mathrm{m}$, point 3: 1.06 $\times 10^{7} \mathrm{~V} / \mathrm{m}$ ) are also slightly increased ( $4.9 \%$ and $\sim 11.3 \%$, respectively). Apparently PMMA filling can reduce the screening effects especially for areas where MWCNTs are closely packed. This may also contribute to the higher emission site density from the PMMA-CNT matrix. However, since these enhancements are moderate, we think that the reduced screening effect is not the only reason for enhanced emission properties described so far.

In fact, a reduced $E_{t h}$ was also detected by Watts et. al, but the mechanism behind was not explained [11]. On the other hand, Tanaka et al. studied the effect of an insulator barrier (amorphous carbon) on individual CNTs [18]. They found that the barrier layer increases the work function and the $E_{t h}$. Apparently the reduced $E_{t h}$ detected from our PMMA-CNT matrix is a different case. Thus we propose the 
following model to explain our results. Consider two MWCNTs a distance $r$ apart from each other. The electric field imposed by an electron located in a nanotube on another electron located at a neighboring nanotube is given by

$$
E=\left(\frac{q}{4 \pi \varepsilon_{0} r^{2}}\right)
$$

where $\varepsilon_{0}$ is the permittivity of vacuum and $q$ is the charge of electrons. When the spacing between these MWCNTs is filled by PMMA with a dielectric constant $k=3.4$, the electric field $\boldsymbol{E}$ will become $\boldsymbol{E}$,

$$
E^{\prime}=\left(\frac{q}{4 \pi \varepsilon r^{2}}\right)=\left(\frac{q}{4 \pi \varepsilon_{0} k r^{2}}\right)
$$

where $\varepsilon=\varepsilon_{0} k$ Hence the electric field between two electrons in two neighboring nanotubes is reduced by the factor of $k=3.4$ for the case of the PMMA-CNT matrix.

Figure 3.11 panels (a)-(b) indicate the schematic drawing of electron flows during electron field emission from the as-grown sample and the PMMA-CNT matrix, respectively.

In this model, we consider that electron flows are driven by the electric forces $\left(F_{E}\right)$ generated by the applied electric field between the anode and the cathode. In addition, we also consider that electric field imposed by electrons in the neighboring MWCNTs will produce a columbic repulsion forces, $\boldsymbol{F}_{\boldsymbol{e}}(=\boldsymbol{q} \boldsymbol{E}, \boldsymbol{E}=$ electric field in equation 1), in directions perpendicular to the driving force $\boldsymbol{F}_{\boldsymbol{E}}$. This means, electron 
flows in individual MWCNT will not simply from the cathode to the anode (upward), but will be drifted left and right along the graphene sheets of the nanotubes due to the
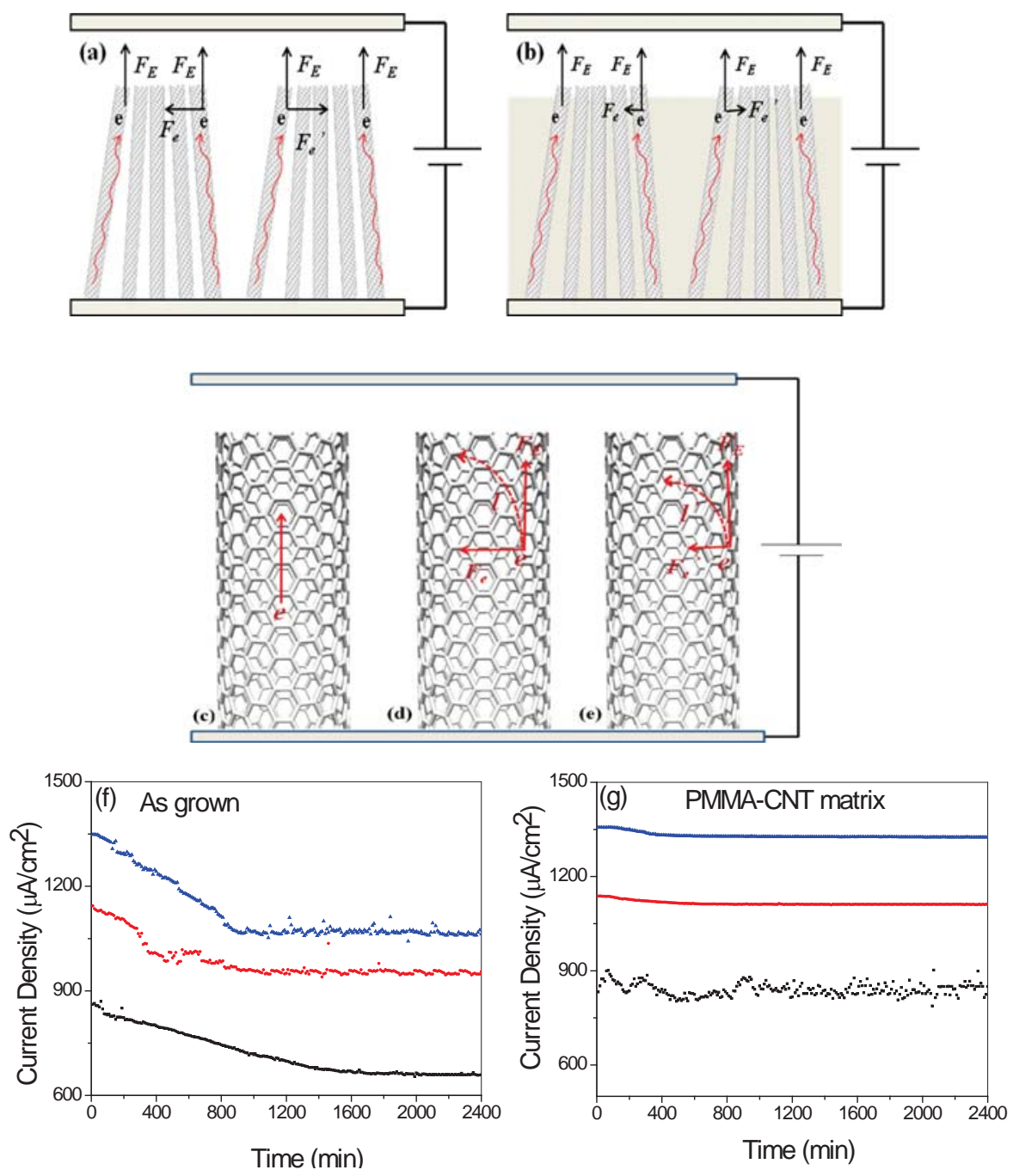

Figure 3.11. Schematic of electron flows in (a) the as grown sample, and (b) the PMMA-CNT matrix. Effective electron paths along (c) an isolated CNT, (d) an as-grown CNT with neighboring nanotubes, and (e) a nanotubes in the PMMA-CNT matrix. Emission current stability curves as a function of time for (f) the as-grown sample and (g) the PMMA-CNT matrix at various initial current densities. Reprinted with permission from [28] 
surrounding columbic forces. For the case of the PMMA-CNT matrix, the drifting will be smaller due to the smaller $\boldsymbol{F}_{\boldsymbol{e}}{ }^{\prime}\left(=\boldsymbol{e} \boldsymbol{E}^{\prime}, \boldsymbol{E}^{\prime}=\right.$ electric field in equation 2). The effective path length travel by electrons before they reached to the tips of nanotubes is explained here. For an isolated nanotube, electrons are driven only by the upward electric forces $\left(\boldsymbol{F}_{\boldsymbol{E}}\right)$ between the anode and the cathode as shown in Figure 3.11 (c).

In this case, the total distance traveled by the electrons before field emission is equal to the length of the nanotube. For nanotube films in our case, neighboring nanotubes are separated by ten of nanometers. In these cases, electric field $\boldsymbol{E}$ imposed by electrons in the neighboring MWCNTs will produce a columbic forces, $\boldsymbol{F}_{\boldsymbol{e}}$, in directions perpendicular to the driving force, as shown in Figure 3.11 (d) and 3.11 (e). For the as-grown sample, electrons flowing in a nanotube will be drifted following the dashed trajectory $(l)$ in Figure 3.11 (d) if there are electrons flowing in the neighboring nanotubes located on the right side. In this case, the actual distance traveled by the electrons before they reached to the tip of the nanotube will be longer than the length of the nanotube. For the PMMA-CNT matrix, electric field $\boldsymbol{E}^{\prime}\left(\left|\boldsymbol{E}^{\prime}\right|<|\boldsymbol{E}|\right)$ will be imposed by electrons in the neighboring nanotubes and produce a columbic force, $\boldsymbol{F}_{\boldsymbol{e}}{ }^{{ }^{\prime}}$ as shown in Figure 3.11 (e). Since $\left|\boldsymbol{F}_{\boldsymbol{e}}\right|<\left|\boldsymbol{F}_{e}\right|$, electrons in this nanotube will travel in a trajectory path shorter $\left(l^{\prime}\right)$ than that in the case of the as-grown sample illustrated in Figure $3.11(d)$.

In reality, all MWCNTs are not defect free. This means, electron flows along MWCNTs will be scattered by defects and generate Joule heating. Thus the longer the electron drifting distance in nanotubes, the more Joule heating will be generated. Due 
to the smaller drifting and shorter effective travel distances, electrons in the PMMACNT matrix will generate less Joule heating. As previously reported, the emission stability depends on the electron scattering process along the nanotubes [19]. For the present study, since electron scattering is reduced for the PMMA-CNT matrices, better field emission stability is expected.

In order to verify this hypothesis, we have evaluated the emission stability of our samples. Figure 3.11 (f) shows the emission stability curves measured from the asgrown sample at different initial current densities $\left(\sim 860, \sim 1140, \sim 1350 \mu \mathrm{A} / \mathrm{cm}^{2}\right)$. As shown, the emission current densities are degraded with time. Figure $3.11(\mathrm{~g})$ shows the typical emission stability curves for the PMMA-CNT matrix. As shown, PMMACNT matrices are stable electron emitters as negligible degradation is detected within the 40-hour continuous test. We think that the improvement in the emission stability of the PMMA-CNT matrix is due to both the reduction in screening effect and electron scattering (Joule heating). As the screening effect from surrounding CNTs is reduced, CNTs in PMMA-CNT matrix can offer more emission sites and thus contribute to higher emission current and lower emission threshold field. The reduced Joule heating means reduced defect scattering along MWCNTs and thus enhance the current stability.

\subsubsection{Field Emission from PMMA-SrTiO}

All samples were characterized by field emission scanning electron microscopy (FESEM) and Raman spectroscopy (laser wavelength $\sim 632 \mathrm{~nm}$, laser spot 
size $\sim 1 \mu \mathrm{m}$ in diameter under a confocal microscope). The STO-CNT samples were also characterized by Transmission Electron Microscopy (TEM) and X-ray Photoelectron Spectroscopy (XPS). The monochromatic focused $A l K_{\alpha} X$-ray (1486.7 eV) source was used for the XPS measurement. The field emission measurements were conducted in a planar diode configuration at a base vacuum pressure of $10^{-8} \mathrm{mbar}$ [19-21]. The spacing between the anode (Indium Tin Oxide/ITO film on glass) and the tips of the VA-MWCNTs was maintained at $1000 \pm 10 \mu \mathrm{m}$ without using dielectric spacer. All measurements were re-confirmed by repeating measurements on the same sample as well as additional sets of samples prepared in the same growth process and treatment.
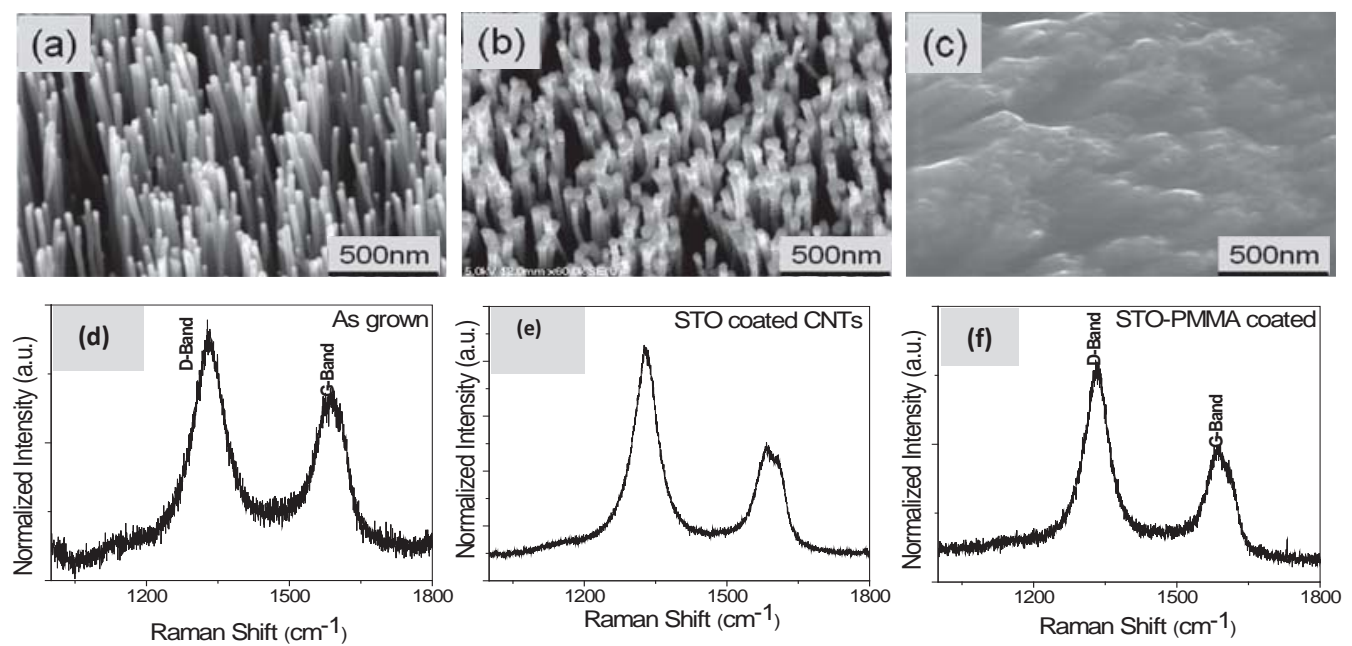

Figure 3.12: SEM images of (a) as grown VA-MWCNTs, (b) STO-CNT sample, and (c) PMMA-STO-CNT sample. Raman spectra of (d) as grown VA-MWCNTs, (e) STO-CNT sample, and (f) PMMA-STO-CNT sample. 
Figure 3.12 (a) shows the SEM image of as-grown VA-MWCNTs. The diameters of these CNTs are $30-50 \mathrm{~nm}$ and their lengths are $\sim 4 \mu \mathrm{m}$. The diameters of the CNTs are larger for the STO-CNT sample as shown in Figure 3.12 (b) indicating successful coating of $\mathrm{SrTiO}_{3}$.

Figure 3.12 (c) shows the typical morphology at the top surface of PMMASTO-CNT matrices. As shown, the tips of CNTs are opened and exposed. Figures 3.12 (d), (e) and (f), are the Raman spectra of the as-grown, STO-CNT, and PMMA-STOCNT samples, respectively. For all samples, the graphitic $(G)$ and defective $(D)$ Raman bands are detected indicating the laser induced Raman scattering occurred on all samples. The $G$ and $D$ bands represent the zone center phonons of $E_{2 g}$ symmetry and the $K$-point phonons of $A_{l g}$ symmetry of the VA-MWCNTs, respectively. The intensity ratio $\left(I_{G} / I_{D}\right)$ for the all samples are merely identical $(\sim 0.89 \pm 0.1)$, indicating similar structural properties of the VA-MWCNTs.

XPS spectra of the as-grown sample (Figure 3.13 (a)) showing the present of $C_{l s}$ signal. Figure 3.13 (b) shows the XPS spectra of the STO-CNT samples. The present of $\mathrm{SrTiO}_{3}$ is confirmed by the composition Sr:Ti:O 1.0:1.0:3.0. Figure 3.13 (c) and (d) showing the TEM images of the as-grown and STO-CNT samples. As shown, the overall thickness of the $\mathrm{SrTiO}_{3}$ coated MWCNT is larger than the typical diameters of our as-grown VA-MWCNTs, consistent with the SEM images in Figure 3.12 (a) and (b). 

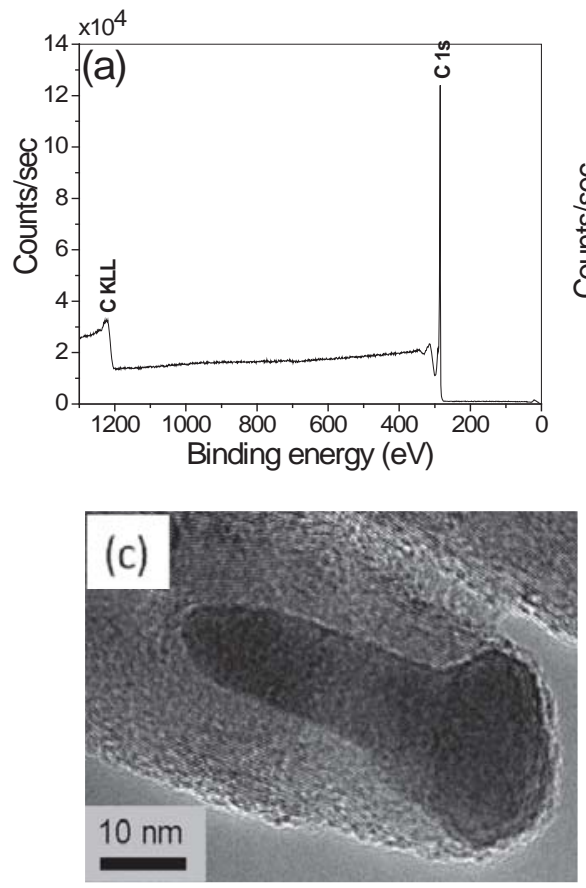
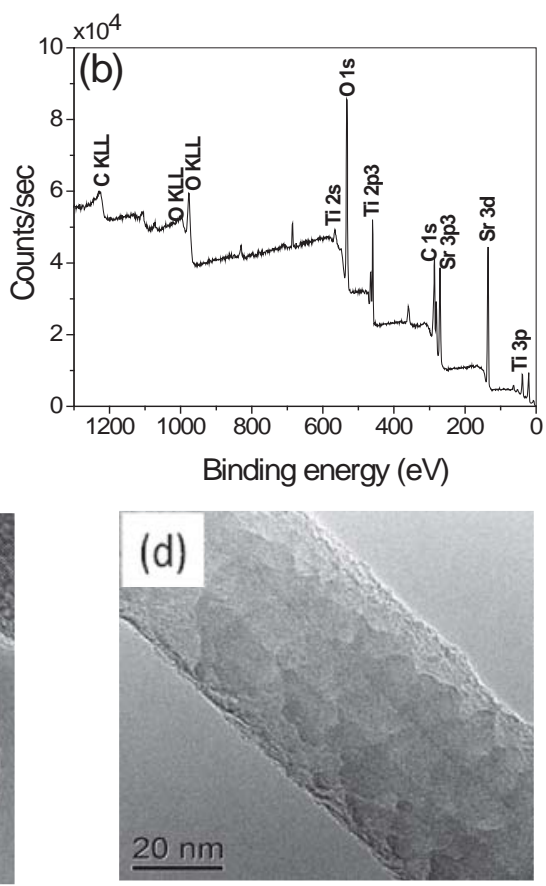

Figure 3.13: XPS spectra and TEM images of the as-grown (a and c) and $\mathrm{SrTiO}_{3}$ coated (b and d) MWCNTs.

Figures 3.14(a), (b), and (c), showing the field emission characteristics [current density $(J)$ versus electric field $(E)$ ] of the as-grown, STO-CNT, and PMMA-STOCNT samples, respectively. The Fowler-Nordheim (FN) equation [22], $J=A \beta^{2} E^{2} \exp$ $\left(-B \Phi^{3 / 2} / \beta E\right)$ is often use to describe electron field emission, where $A, B$ are constants, $E$ is the applied electric field in $\mathrm{V} \mathrm{cm}^{-1}$, and $\Phi$ is the work function of the emitters in $\mathrm{eV}, \beta$ is the field enhancement factor. Insets of these figures showing the corresponding linear F-N plots, which verified that the detected currents are due to quantum tunneling. As shown, the threshold electric field, $E_{t h}$ (applied electric field for generating a current density of $1 \mu \mathrm{A} / \mathrm{cm}^{2}$ ) is $3.8 \mathrm{~V} / \mu \mathrm{m}, 2.0 \mathrm{~V} / \mu \mathrm{m}$ and, and 0.8 
$\mathrm{V} / \mu \mathrm{m}$ for the as-grown, STO-CNT, and PMMA-STO-CNT samples, respectively. This means, STO coating can reduce $E_{t h}$ of the as-grown VA-MWCNTs. Local removal of the $\mathrm{SrTiO}_{3}$ coating at the tips of VA-MWCNTs have further reduced the $E_{t h}$ of the PMMA-STO-CNT sample. While we can explain the reduced $E_{t h}$ of the STO-CNT sample by the reduced work function of the $\mathrm{SrTiO}_{3}$ coating, however, it is not clear why the opened tip PMMA-STO-CNT sample shown further reduction of $E_{t h}$ after the removal of $\mathrm{SrTiO}_{3}$ coatings.

To further investigate our observation, we have examined the emission site densities as obtained from the fluorescence on the ITO electrode due to electron bombardment. As shown, the emission density for the as-grown sample is poor (inset of Figure 3.14 (a)). This is due to screen effect in the CNT forest as consistent with our previous observation [16]. Emission density from the STO-CNT sample (inset of Figure 3.14 (b)) is higher. This cannot be solely explained by the reduced work function as this will mainly increase the current density from each emitter and not increasing the number of emitters in the STO-CNT sample. This means, the $\mathrm{SrTiO}_{3}$ coating has also reduced the screening effect.

Now, let us examine the effect of removing the $\mathrm{SrTiO}_{3}$ coated from the CNT tips. As shown in the inset of Figure 3.14 (c), the emission density of the PMMASTO- CNT matrix is even higher than that of the STO-CNT sample. This means, the filling of STO-CNT with PMMA followed by the exposure of the CNT tips have 

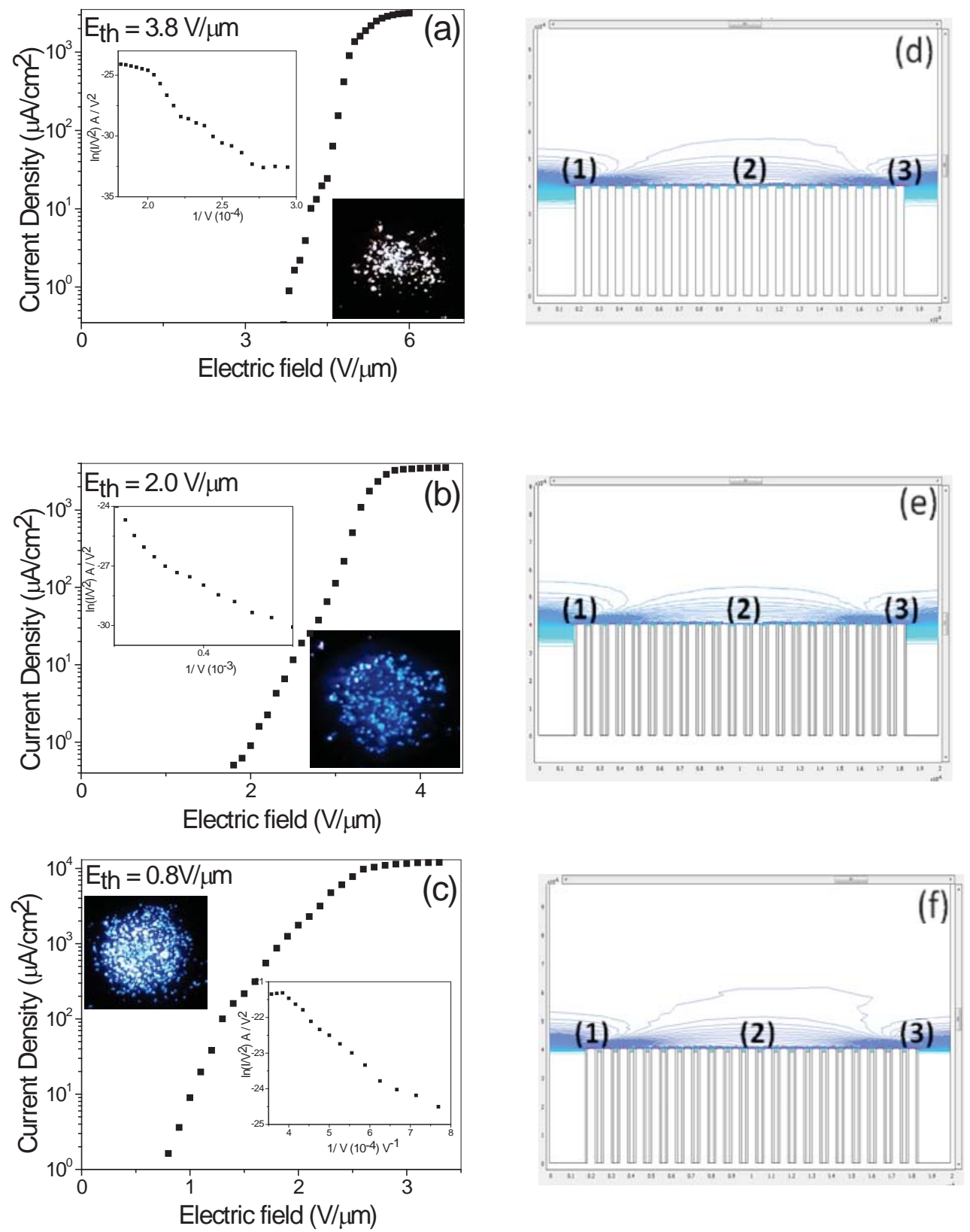

Figure 3.14: Electron field emission properties $(a, b$, and $c)$ and the simulated potential maps ( $d$, e, and f) of the as-grown ( $a$ and d), STO-CNT ( $b$ and e) and PMMA-STO-CNT (c and $f$ ) samples.

further reduce the screening effect in the matrix. In short, PMMA-STO-CNT matrix offers lowest $E_{t h}$ and highest emission density. 
Since, the distance between anode-cathode was $\sim 1 \mathrm{~mm}$; the actual density would have been higher at distance closer to the sample surface. Form the sizes of the sample and the overall fluorescence image, the divergence angle of the emission was estimated as $23.5^{\circ}$.

We have then simulated and compared the local electric field strength at the tips of CNTs for all samples by the COMSOL Multiphysics software. This will allow us to understand the effect of conformal $\mathrm{SrTiO}_{3}$ coating on the whole surfaces of MWCNTs, as well as the local removal of these $\mathrm{SrTiO}_{3}$ coating at the tips of the nanotubes. The simulation parameters are as follows: diameter of CNTs, $D_{C N T s}=40$ $\mathrm{nm}$; Length of CNTs, $L_{C N T s}=4 \mu \mathrm{m}$; edge to edge spacing between CNTs, $S=60 \mathrm{~nm}$; applied electric field between top and bottom boundaries, $E_{a p p l}=5 \mathrm{~V} / \mu \mathrm{m}$. The simulated potential map for an array of a-grown VA-MWCNTs is shown as Figure 3.14 (d). As shown, the CNT located at the center of the array (point 2) experienced highest screening effect from the surrounding nanotubes and thus posses lowest electric field $\left(\sim 9.898 \times 10^{6} \mathrm{~V} / \mathrm{m}\right.$ at point $1, \sim 5.326 \times 10^{6} \mathrm{~V} / \mathrm{m}$ at point 2 and $\sim 9.97 \times$ $10^{6} \mathrm{~V} / \mathrm{m}$ at point 3, respectively). The effect of $\mathrm{SrTiO}_{3}$ coatings was simulated by setting the 20-nm of the spacing next to the surfaces of each CNT with a dielectric constant $\boldsymbol{k} \sim 475$ (instead of vacuum $\boldsymbol{k} \sim 1$ ). The potential map for the simulated STOCNT sample is illustrated in the Figure 3.14 (e). Again, CNT at the center (point 2) experienced largest screening effect and lowest local field $\left(\sim 8.878 \times 10^{6} \mathrm{~V} / \mathrm{m}\right.$ at point 2). This local field is increased by $\sim 66.7 \%$ as compared to that in the as-grown 
sample. The local fields at the tips of CNTs located at the edges of the array $(\sim 1.559 \mathrm{x}$ $10^{7} \mathrm{~V} / \mathrm{m}$ at point 1 , and $\sim 1.119 \times 10^{7} \mathrm{~V} / \mathrm{m}$ at point 3 ) are also slightly increased by $\sim 57.5 \%$ and $12.2 \%$, respectively. These are due to the reduced screening effect as the "vacuum" surrounding the CNTs is now partially replaced by " $\mathrm{SrTiO}_{3}$ coatings" that has much higher dielectric constant. Simulation for the PMMA-STO-CNT sample was conducted by setting the remaining vacuum spacing between the STO-CNT with PMMA coatings (dielectric constant, $\boldsymbol{k}=3.4$ ) [24]. In this case, only the sides of the CNTs are simulated with the $\mathrm{SrTiO}_{3}$ coatings but not at the top surface of the nanotubes tips. As shown in Figure 3.14 (f), the local field at the center of the PMMASTO-CNT matrix ( $\sim 9.366 \times 10^{6} \mathrm{~V} / \mathrm{m}$ at point 2$)$ is now $\sim 75.9 \%$ high than that in the as-grown sample due to the additional PMMA filling. The local fields at the edges of the matrix $\left(\sim 1.298 \times 10^{7} \mathrm{~V} / \mathrm{m}\right.$ at point 1 and $\sim 1.155 \times 10^{7} \mathrm{~V} / \mathrm{m}$ at point 3$)$ are also found to be slightly increased ( $\sim 31.1 \%$ and $\sim 15.9 \%$, respectively).

From these simulations, it is obvious that $\mathrm{SrTiO}_{3}$ coating can reduce the screening effects especially for areas where MWCNTs are closely packed. Consequently, the reduced screening effect has the contribution towards lower $E_{t h}$ and higher emission site density of STO-CNT sample detected here. However, the additional filling of PMMA did not further enhance the local field on the fully filled PMMA-STO-CNT sample. This is expected since the dielectric constant of PMMA is two-order of magnitude smaller than that of $\mathrm{SrTiO}_{3}$ while their thicknesses on PMMA-STO-CNT sample are similar. In fact, there is one factor which was not 
considered in the simulation, i.e., the effect of work function $(\Phi)$ on the tunneling process. Although coatings of wide band gap materials were in the past claimed to reduce the work function of CNTs and allow easier electron field emission. However, Tanaka et al. studied the effect of an insulator barrier (amorphous carbon) on individual CNTs [25]. They found that the barrier layer increases $\Phi$ and the $E_{t h}$. If this is true, the removal of PMMA and $\mathrm{SrTiO}_{3}$ coatings from our PMMA-STO-CNT will reverse our sample with the original CNT work function while having reduced screening effects from the PMMA and $\mathrm{SrTiO}_{3}$ coatings at spaces between CNTs. This means, $\mathrm{SrTiO}_{3}$ coatings on our STO-CNT sample improve the performance of CNTs by reducing the screening effect more than reducing the work function. The exposure of the CNT tips thereafter retains the reduced screening effect and removes the barrier layer. Of course, we cannot totally rule out the possibility that our CNT tips are not fully exposed. They may have a very thin layer of $\mathrm{SrTiO}_{3}$ coatings at the tips. Since such coating is thin, the added potential barrier is thin and still allows effective band bending and tunneling process.

While we have explained our observation in Figures 3.14 (a), (b) and (c), the enhanced local field due to the reduced screening effect is not really significant according to our simulation in Figures 14 (d), (e) and (f). Therefore, we are putting forward the following model to explicate our results. In the present model, we are considering two MWCNTs with a distance $r$ apart from each other. The electric field imposed by an electron located in a nanotube on another electron located at a 
neighboring nanotube is given by equation (1). When the spacing between these MWCNTs is filled by materials with a dielectric constant $\boldsymbol{k}$, the electric field is given by the equation (2). Hence the electric field between two electrons in two neighboring nanotubes is reduced by the factor of $\boldsymbol{k}$. In principle, if we fill up the spaces between VA-MWCNTs by $\mathrm{SrTiO}_{3}$, Coulomb repulsion forces between electrons located at adjacent CNTs in such a STO-CNT sample will be reduced by a factor as high as 475time.

Assuming that such a fully-filled STO-CNT sample exists, electron flows on CNTs during field emission can be represented by Figures 3.11 (a) and (b). In the current model, electron flows along CNTs are driven by the electric forces $\left(\boldsymbol{F}_{\boldsymbol{E}}\right)$ generated by the applied electric field between the anode and the cathode. In addition, we assume that columbic repulsion forces, $\boldsymbol{F}_{\boldsymbol{e}}(=\boldsymbol{q E}, \boldsymbol{E}=$ electric field in equation 1), are generated by electrons in the neighboring CNTs in directions perpendicular to the driving force $\boldsymbol{F}_{\boldsymbol{E}}$. This means, electrons will not simply from the cathode to the anode (upward) but will be drifted left and right on the graphene sheets of the nanotubes due to the surrounding Columbic forces (Figure 3.11 (a)). In the case of a fully-filled STOCNT sample, the drifting will be smaller due to the smaller repulsion forces $\boldsymbol{F}_{\boldsymbol{e}}{ }^{\prime}\left(=\boldsymbol{e} \boldsymbol{E}^{\prime}\right.$, $\boldsymbol{E}^{\prime}=$ electric field in equation 2).

Let us consider the effective path length travel by electrons under different Coulomb repulsion forces. Figure 3.11 (c) represents the case of an isolated nanotube, where the upward electric force $\left(\boldsymbol{F}_{\boldsymbol{E}}\right)$ between the anode and the cathode is the only 
driving force for the electron flow. In this case, an electron will travel by a distance equal to the length of the nanotube prior to field emission.

Figure 3.11 (d) represents the case of a nanotube within a typical as-grown sample. In this case, electric field $\boldsymbol{E}$ imposed by electrons in the neighboring CNTs will produce repulsion forces, $\boldsymbol{F}_{\boldsymbol{e}}$, in directions perpendicular to the driving force. This will cause drifting of the electron following the dashed trajectory $(l)$ if there are electrons flowing in the neighboring nanotubes located on the right side. Thus, the distance travelled by the electron will be longer than the length of the nanotube before they reached to the tip of the nanotube. For a nanotubes located in a fully-filled STOCNT sample, electric field $\boldsymbol{E}^{\prime}\left(\left|\boldsymbol{E}^{\prime}\right|<|\boldsymbol{E}|\right)$ will be imposed by electrons in the neighboring nanotubes and produce repulsion forces, $\boldsymbol{F}_{\boldsymbol{e}}{ }$ as shown in Figure 3.11 (e). Since $\left|\boldsymbol{F}_{\boldsymbol{e}}{ }^{\prime}\right|<\left|\boldsymbol{F}_{\boldsymbol{e}}\right|$, electrons in this nanotube will travel in a trajectory path shorter $\left(l^{\prime}\right)$ than that in the case of the as-grown sample illustrated in Figure 3.11 (d).

In practical, CNTs are not defect free. This means, electron flows along MWCNTs will be scattered by defects and generate Joule heating. Due to the shorter effective path length travel by electrons, Joule heating in a fully-filled STO-CNT sample is expected to be lower as compared to the case for an as-grown sample. As reported in our earlier papers, electron scattering and Joule heating are responsible for poor emission stability. In fact, we have attempted to fully fill up the spaces between CNT by $\mathrm{SrTiO}_{3}$ coating to match the simulated STO-CNT sample. However, thicker 

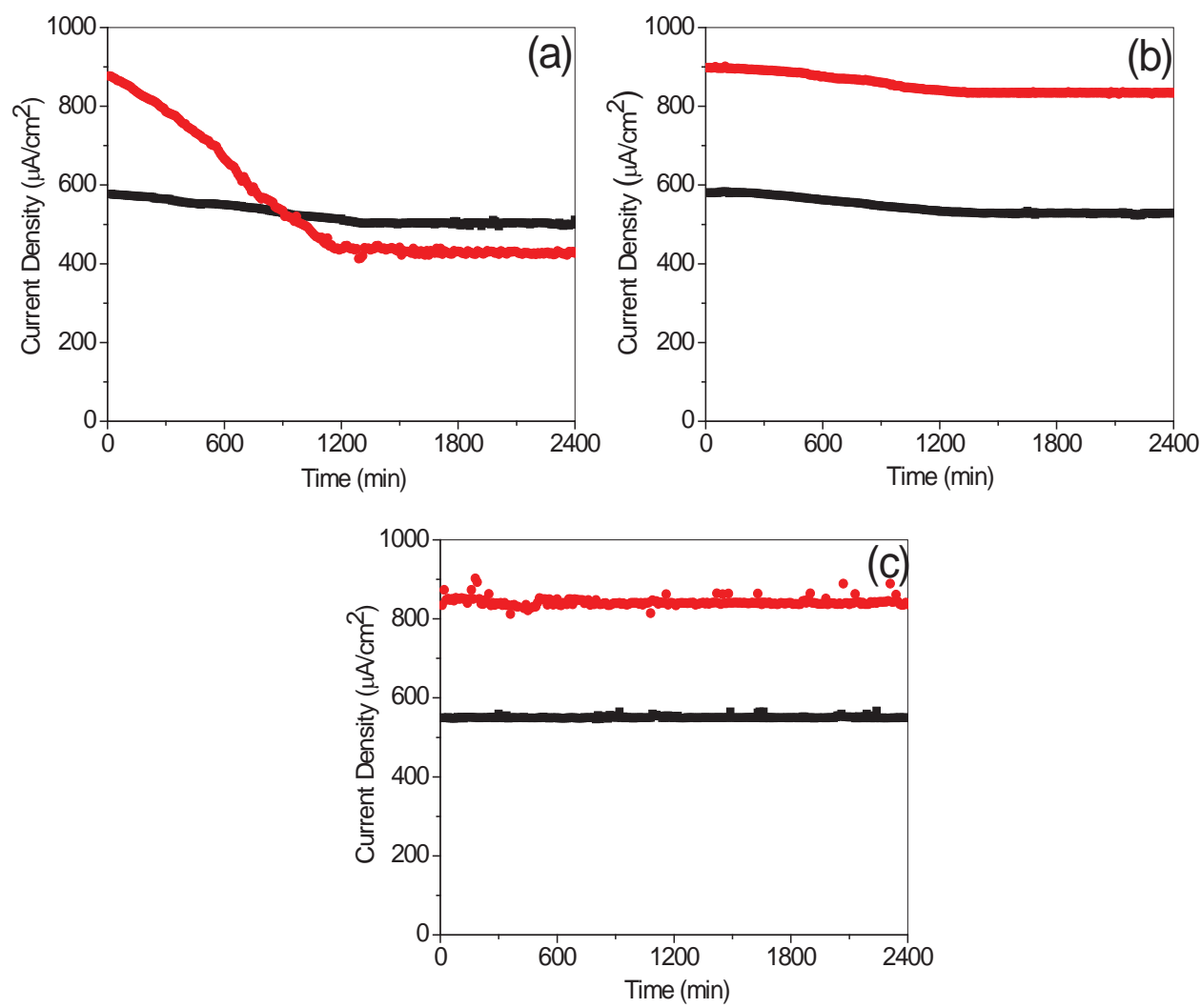

Figure 3.15: Emission current stability curves for (a) the as grown, (b) the STO-CNT sample, and (c) the PMMA-STO-CNT matrix.

$\mathrm{SrTiO}_{3}$ coating results in a brittle and void structure and cannot be use for our experiments. We have thus decided to fill up the spaces in our STO-CNT sample by PMMA, which has enabled the tip removal experiments described so far. According to our model, the coating of $\mathrm{SrTiO}_{3}$ in the STO-CNT sample and the PMMA-STO-CNT matrix can reduce electron scattering and Joule heating and thus are expected to emit electron with high stability.

In order to verify the above mentioned hypothesis, we have evaluated the emission stability of our samples. Figures 3.15 (a), (b), and (c) show the changes of 
emission current density with time for the as-grown sample, the STO-CNT sample, and the PMM-STO-CNT matrix. All samples were tested with two different initial current densities ( $\sim 600$ and $\left.\sim 880 \mu \mathrm{A} / \mathrm{cm}^{2}\right)$. As shown in Figure 3.15 (a), the emitted current from the as-grown sample were degraded with time, especially at higher tested current density. The STO-CNT sample show very stable emission current with time, $\sim 5-8 \%$ degradation after 2,400 min testing (Figure 3.15 (b)). The PMMA-STO-CNT matrix shows remarkable emission stability without significant degradation after the continuous 40-hour test.

\subsection{Conclusions}

In conclusions, we were able to demonstrated different ways to improve the field emission from as grown CNTs. In summary,

1) We found that opened-tip VA-MWCNTs can produce more stable emission. Bundling of these VA-MWCNTs can further reduce the screening effects, increase the emission density, and improve the emission stability. These results are confirmed by theoretical simulation, which further suggests that a two-order of magnitude lower electric field loading are applied on these bundles that reduce current loading, thermal and mechanical stresses and thus enhance the emission stability.

2) We found that PMMA-CNT matrices are excellent electron field emitters. The emission threshold field for $1 \mu \mathrm{A} / \mathrm{cm}^{2}$ was reduced to $1.675 \mathrm{~V} / \mu \mathrm{m}$ in comparison with $3.898 \mathrm{~V} / \mu \mathrm{m}$ of the as-grown samples. The emission densities from the 
PMMA-CNT matrices are high, merely filled up the entire growth surface of the sample. We discussed the possible reasons behind this field emission improvement and attributed these enhancements to 1) the reduction in the screening effect from the neighboring CNTs due to dielectric properties of PMMA as suggested by our theoretical simulation, and 2) reduced defect scattering and Joule heating due to the shorter effective transport distance of the electrons in MWCNTs. We have demonstrated stable emission at a level of $1350 \mu \mathrm{A} / \mathrm{cm}^{2}$ continuously for 40 hours. It is evidenced here that filling the spaces between CNTs with dielectric materials will lead to lower $E_{t h}$, higher emission density, and better emission stability. This approach can be a promising method for fabricating practical electron field emitters.

3) Field emission properties of as-grown VA-MWCNTs were improved due to the reduced screening effect between $\mathrm{CNTs}$ after the coating of $\mathrm{SrTiO}_{3}$ that has high dielectric constant. Such an enhancement on our STO-CNT samples can be further improved by the exposure of the CNT tips after PMMA filling and mechanical polishing. The Field emission threshold electric field decreased from $3.8 \mathrm{~V} / \mu \mathrm{m}$ in the as-grown sample to $\sim 0.8 \mathrm{~V} / \mu \mathrm{m}$ for the PMMA-STO-CNT sample. We propose that the coating of $\mathrm{SrTiO}_{3}$ could have also reduce the Coulomb repulsion forces between electrons in neighboring CNTs. This has lead to lower drifting of electron flows in CNTs and thus reduce electron-phonon scattering and Joule heating. Such a shorter effective transport distance of the electrons in CNTs helps improve the emission stability as proven later experimentally. 


\subsection{Future work on Field Emission}

A lot of progress has been demonstrated towards the enhancement of field emission parameters from carbon nanotubes (CNTs) using different techniques. This is an ongoing work and a lot more can be done. For example

1) One important study is variation in field emission properties of the device at low/poor vacuum. Another area to explore is for making flexible field emission devices by transferring as grown CNTs on a flexible substrate and studying or enhancing its field emission behavior.

2) For any working devices to be applicable in real world the stability of CNT based devices should be more than 1000 hours. Therefore devices must be tested for more than longer hours.

3) Different and higher dielectric materials can also be tested for coating CNTs for longer stability and better field properties.

4) Also in-situ studies of field emission from a single CNT STM inside TEM can also help us unravel the change in structure of CNTs during field emission process.

\subsection{References}

1. S. Ijima, Helical microtubules of graphitic carbon. Nature (London), 1991, 354, 56.

2. M. M. J. Treacy, T. W. Ebbesen and J. M. Gibson. Exceptionally high young's modulus observed for individual carbon nanotubes, Nature (London), 1996, 381,678 . 
3. M. S. Dresselhaus, G. Dresselhaus, P. Avouris. Carbon nanotubes: synthesis, structure, properties, and application, Springer, Berlin, 2001, 287.

4. D. A. Walters, L. M. Ericson, M. J. Casavant, J. Liu, D. T. Colbert, K. A. Smith, R. E. Smalley. Elastic strain of freely suspended single-wall carbon nanotube ropes, Appl Phys Lett., 1999, 74, 3803.

5. W. A. deHeer, A. Chatelain and D. Ugarte. A carbon nanotube field-emission electron source, Science, 1995, 270, 1179.

6. P. G. Collins, A. Zettl. A simple and robust electron beam source from carbon nanotubes, Appl. Phys. Lett, 1996, 69, 1969.

7. W. B. Choi, D. S. Chung, J. H. Kang, H. Y. Kim, Y. W. Jin, I.T. Han, Y. H. Lee, J. E. Jung, N. S. Lee, G. S. Park, J. M. Kim. Fully sealed, high-brightness carbon-nanotube field-emission display, Appl Phys Lett, 1999, 75, 3129.

8. L. Nilsson, O. Groening, C. Emmenegger, O. Kuettel, E. Schaller, L. Schlapbach et al., Scanning field emission from patterned carbon nanotube films. Appl Phys Lett, 2000, 76, 2071.

9. J. M. Bonard, C. Klinke, K. A. Dean, B.F. Coll. Degradation and failure of carbon nanotube field emitters, Phys Rev B, 2003, 67, 115406.

10. I. T. Han, H. J. Kim, Y. Park, N. Lee, J. E. Jang, J.W. Kim et al., Fabrication and characterization of gated field emitter arrays with self aligned carbon nanotubes grown by chemical vapor deposition, Appl Phys Lett, 2002, 81, 2070.

11. Y. Cheng, O. Zhou. Electron field emission from carbon nanotubes, C. R. Physique, 2003, 4, 1021. 
12. D. W. Kim, S. Jin. Morphology control of patterned carbon nanofiber arrays for field emission applications. J. Appl. Phys. 2006, 45, 346.

13. S. P. Patole, A. S. Patole, D. S. Rhen, M. Shahid, H. Min, D. J. Kang, T. H. Kim, J. B Yoo, Patterned carbon nanotube growth using an electron beam sensitive direct writable catalyst, Nanotechnology 2009, 20, 315302.

14. W. P. Kanga, J. L. Davidsona, A. Wisitsora-ata, Y. M. Wonga, R. Takalkara, K. Subramaniaa, D. V. Kernsb, W. H. Hofmeister, Diamond and carbonderived vacuum micro- and nano-electronic devices, Diamond Relat. Mater, $2005,14,685$.

15. Q. H. Wang, T. D. Corrigan, J. Y. Dai, R. P. H. Chang, A. R. Krauss, Appl. Phys. Lett., 1997, 70, 3308.

16. A. Pandey, A. Prasad, J. Moscatello, B. Ulmen, Y. K. Yap, Enhanced field emission stability and density produced by conical bundles of catalyst-free carbon nanotubes, Carbon, 2010, 48, 287.

17. J. M. Bonard, J. P. Salvetat, T. Stöckli, L. Forr'o, A. Châtelain. Field emission from carbon nanotubes: perspectives for applications and clues to the emission mechanism, Appl. Phys. A, 1999, 69, 245.

18. S. Fan, M. G. Chapline, N. R. Franklin, T. W. Tombler, A. M. Cassell, H. Dai. Self-oriented regular arrays of carbon nanotubes and their field emission properties, Science, 1999, 283, 512

19. M. Schvartzman, A. Mathur, Y. Kang, C. Jahnes, J. Hone, S. J. Wind. Fluorinated diamond like carbon templates for high resolution nano imprint lithography. J. Vac. Sci. Technol. B, 2008, 26, 6. 
20. W. Yi, T. Jeong, S. G. Yu, J. Heo, C. Lee, J. Lee, W. Kim, J. B. Yoo, J. Kim, Field emission characteristics from wide-bandgap material-coated carbon nanotubes. Adv. Mater., 2002, 14, 1464.

21. P. C. P. Watts, S. M. Lyth, E. Mendoza, S. R. P. Silva, Polymer supported carbon nanotube arrays for field emission and sensor devices. Appl. Phys. Lett. 2006, 89, 103113.

22. S. H. Jo, Y. Tu, Z. P. Huang, D. L. Carnahan, D. Z. Wang, Z. F. Ren. Effect of length and spacing of vertically aligned carbon nanotubes on field emission properties. Appl. Phys. Lett., 2003, 82, 3520.

23. F. Jin, Y. Liu, C. M. Day, S. A. Little, Enhanced electron emission from functionalized carbon nanotubes with a barium strontium oxide coating produced by magnetron sputtering, Carbon, 2007, 45, 587.

24. J. M. Bonard, C. Klinke, C. Degradation and failure of carbon nanotube field emitters. Phys. Rev. B, 2003, 67, 115406.

25. V. K. Kayastha, B. Ulmen, Y. K. Yap, Effect of graphitic order on field emission stability of carbon nanotubes. Nanotechnology, 2007, 18, 035206-1.

26. L. Nilsson, O. Groening, C. Emmenegger, O. Kuettel, E. Schaller and L. Schlapbach et al., Scanning field emission from patterned carbon nanotube films, Appl Phys Lett., 2000, 76, 2071.

27. J. Menda, B. Ulmen, L. K. Vanga, V. K. Kayastha, Y. K. Yap, Z. Pan et al., Structural control of vertically aligned multiwalled carbon nanotubes by radio-frequency plasmas, Appl Phys Lett., 2005, 87, 173106-1.

28. A. Pandey, A. Prasad, J. P. Moscatello, Y. K. Yap. Stable electron field emission from PMMA-CNT matrices, ACS Nano, 2010, DOI: 10.1021/nn100925g 


\section{Chapter 4}

\section{Glucose Biosensors Based on PMMA-CNT Matrices}

\subsection{Introduction: Basics of Biosensors}

A biosensor is a device which utilizes a biochemical reaction to produce a biological response and convert them to an electrical signal. Usually, a biological response/signal cannot be examined visually therefore it has to be converted into a signal which can be easily detected. We use electrochemical techniques to study the reduction-oxidation (redox) reactions at the electrode-solution interface which convert biological signals into detectable electrical signal. Electrochemical techniques are methods that study reactions at the electrode-solution interface.

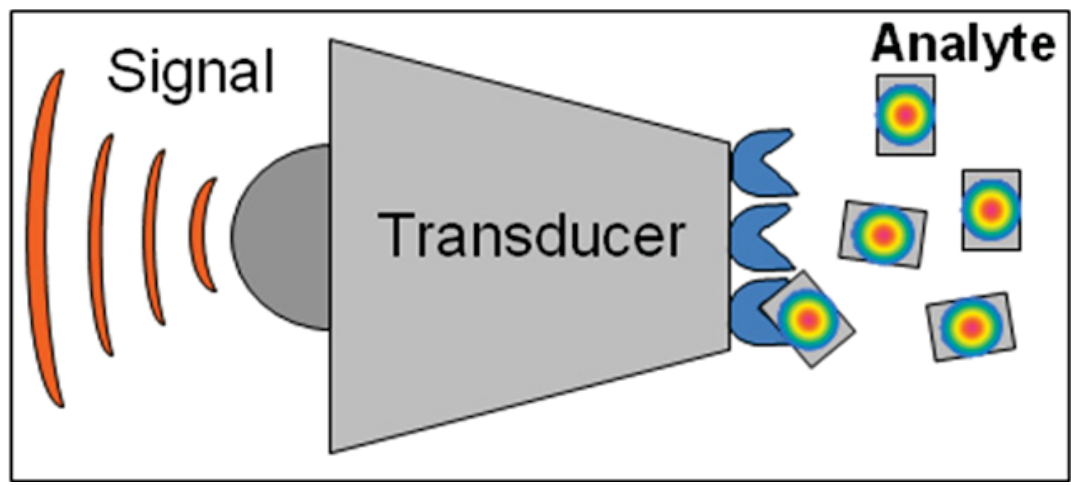

Figure 4.1: Components of biosensor 
In analytical electrochemistry, biocatalyst and transducer constitute a biosensor, which is also considered as an electrode. Transducer is the main component which converts the response to measurable electrical signals. Then, the amplifier, processor and display (as analytical electrochemical equipment) convert these signals to detectable signals.

\subsubsection{Components of biosensors}

Biosensors have three major components: biological element which detects the analyte, transducer and signal processors. Biological element can be complex proteins, peptides, glucose or any other complex molecules. The transducer is a device which converts the biological signal into measurable electrical signal. Figure 4.1 schematically shows the components of a biosensor. For glucose biosensor, the glucose oxidase $\left(G O_{x}\right)$ enzyme is the biological element that detects the analyte (glucose molecules). Here, CNTs act as the transducer and electrochemical cell setup and potentiostat is the signal processor. Biosensors can be classified in many different ways [1]. If sorted according to biocatalyst, biosensors are classified into enzyme electrodes, immune-sensors, DNA sensors and microbial sensors etc. If categorized by transducers, they can be sorted into electrochemical sensors, electrical sensors, optical sensors, mass sensitive sensors and thermal sensors based on signal transduction [2]. 


\subsubsection{Glucose oxidase $\left(G O_{x}\right)$}

Glucose oxidase was first discovered by Müller [3]. It was established in 1928 that the enzyme glucose oxidase acts as a catalyst and causes the oxidation of glucose to gluconic acid in the presence of dissolved oxygen [4-5]. The structure of $G O_{x}$ molecule is illustrated in Figure 4.2.

$G O_{x}$ is a dimeric protein. Dimeric consists of two similar subunits called monomers. The molecular weight of $G O_{x}$ is $\sim 160 \mathrm{kDa}(1 \mathrm{Da}=1 \mathrm{~g} / \mathrm{mol})$ with one Flavin adenine dinucleotide (FAD) per monomer as a cofactor. Co-factors are nonprotein in nature and they are bound to a protein for its biological activities [6]. The monomers are connected via non-covalent bond. The monomeric molecule is a compact spheroid with approximate dimensions of $60 \AA$ x $52 \AA$ x $37 \AA$.

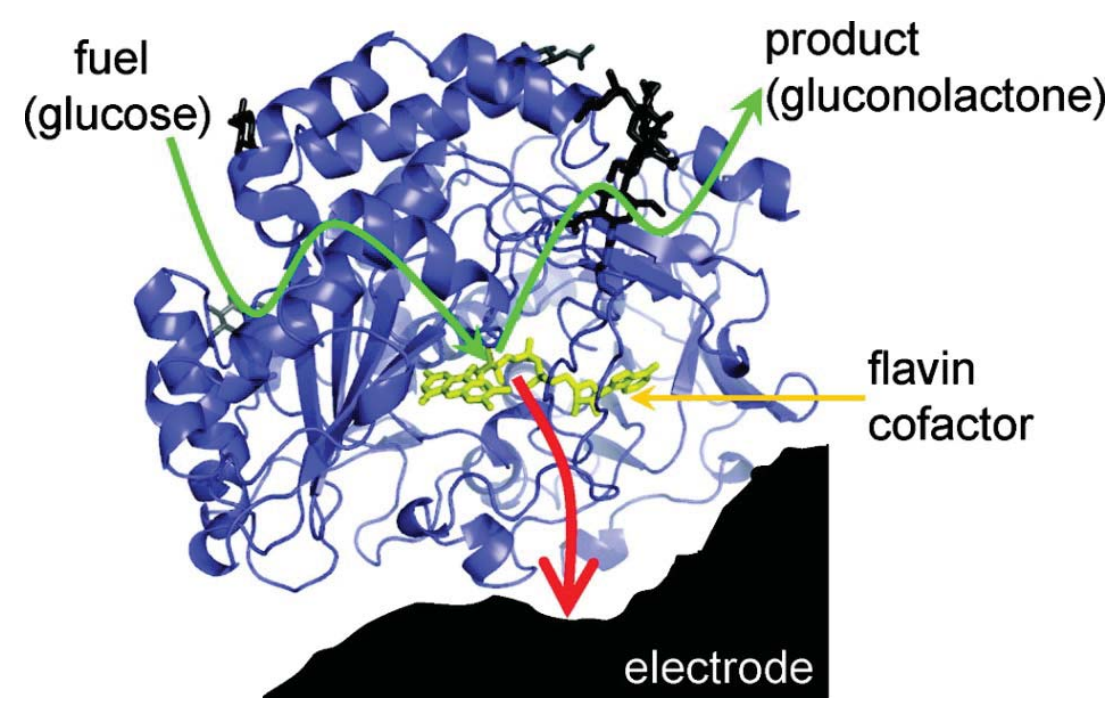

Figure 4.2: Overall topography of GOx enzyme with FAD at the center. Reprinted with permission from reference [7] 
FAD is part of $G O_{x}$ where redox reaction takes place. This $\mathrm{FAD} / \mathrm{FADH} \mathrm{H}_{2}$ redox center is encapsulated deep inside the protein shell. Therefore the reactive enzyme site

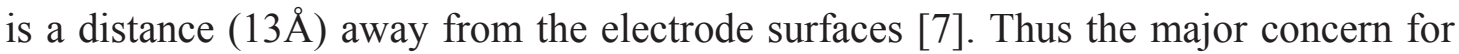
fabricating glucose biosensors is to establish an efficient direct electron transfer (DET) between the FAD center and electrode surface.

\subsection{Current state of research in biosensors based on CNTs}

Diabetes mellitus is one of the foremost causes of various neurovascular and retinopathy diseases. The survey of world health organization (WHO) shows that the sole therapy which can be used as a preventive technique is not to raise the blood glucose level i.e. apposite control of the blood glucose as a clinical diabetes therapy [8-9]. There are many blood glucose level detection techniques available in market which comprises offline detection of blood glucose by extracting blood from the capillaries or veins of the fingertip such as commercially available blood glucose monitors. For many diabetic patients continuous monitoring of blood glucose is required. The existing technologies in market for continuous glucose monitoring are few and expensive. Biosensor based on carbon nanotubes has been proved to be a more reliable and promising to be used for continuous glucose application which represents a most conceivable and exciting application area for nano-biotechnology. These biosensors can be embedded in the body of the patient which will act as a 
regular glucose monitoring system because biosensors are approved sensitive, selective and responsive medium.

Carbon nanotubes possess excellent chemical and physical properties to be used as biosensor as demonstrated in literature [10-16]. While preparing the CNTs based glucose biosensors, the most common and extensively used enzyme, glucose oxidase $\left(G O_{x}\right)$, is amalgamated with CNTs [17-20]. Since, CNTs possess the incredible electro- catalytic and exhibit fast electron transfer rate therefore, it is a common assumption that CNTs can capture the electrons from the deeply embedded FAD redox centres in $G O_{x}$ and act as transducers in CNTs based glucose biosensors [21]. For the fabrication of biosensors, Wang et al. [21] used CNTs as an electrode material. Simultaneously they filled the mixture of CNTs and $G O_{x}$ in a polyamide tube and sealed the other end with the nafion coating and recorded the current response of the sensor [21]. Since then various techniques such as cross-linking [22], physical adsorption [23], and decoration of CNT modified glassy carbon electrode (GCE) with metallic nanoparticles [24-25] etc. has been used to improve the immobilization of $G O_{x}$ onto surface of different electrodes including CNTs. However, these techniques are complicated and involve non-compatible reagents which produce biosensors that do not exhibit good stability and longer life time. Although stability, sensitivity and low limit of detection and response time of CNTs based biosensors are important, there are not many reports discuss about durability, reusability, and lifetime of $G O_{x^{-}}$ CNTs based biosensors. In most cases, the dispersion of CNTs (which is the key 
element for making CNT based biosensors) affects the immobilization of enzyme and limits its performance. It is evident that as grown CNTs have closed shell and it does not allow high degree of functionality. Keeping this in mind Lin et al. employed open end CNTs and fabricated glucose biosensors based on aligned CNT nanoensembles [26]. The glucose biosensor based on CNTs nanoensembles showed highly selective detection of glucose and maintained a linear response up to a glucose concentration of $20 \mathrm{mM}[26]$.

For real application of these CNTs-based glucose biosensors, it is important to study the durability, reusability and stability of the $G O_{x}$ enzyme. This chapter reports a durable glucose biosensors based on $G O_{x}$-PMMA-CNT matrices. We found that PMMA-CNT matrices are reliable electrodes to immobilize $G O_{x}$ for sensors that are sensitive, robust and durable. The reusability factor of our sensors was also evident when we kept these sensors for longer than eight months in proper storage conditions. Furthermore, we found that our sensors have a glucose reaction rate of $0.0104 \mathrm{nmol} / \mathrm{seconds}$. Details of these results are discussed in this chapter.

\subsection{Experimental procedure: Fabrication of glucose biosensors based on PMMA- CNT matrix}

\subsubsection{Growth of VA-MWCNTs}

VA-MWCNTs were grown using plasma enhanced chemical vapor deposition technique (PECVD). In brief, Ni nanoparticles were deposited using magnetron 
sputtering system on silicon $(\mathrm{Si})$ substrates. VA-MWCNTs were then grown by PECVD using pure $\mathrm{CH}_{4}$ gas.
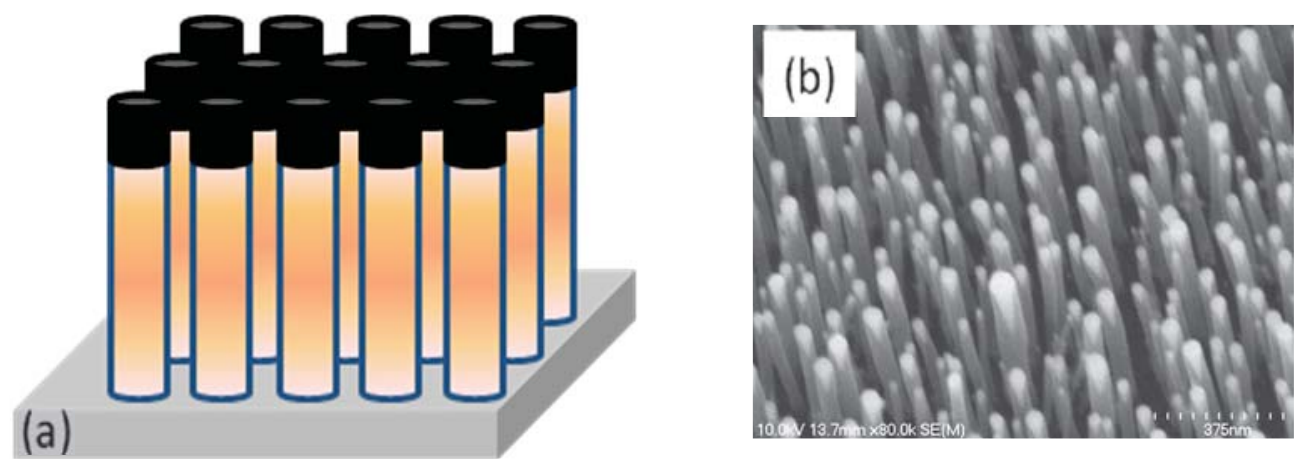

Figure 4.3: (a) Schematic diagram of as grown vertically aligned multi-walled CNTs (VAMWCNTS) (b) corresponding SEM image of as grown VA-MWCNTs with Ni catalyst on top

Figure 4.3(a) shows the schematic diagram of as grown VA-MWCNTs. The corresponding scanning electron microscopic (SEM) image of as grown VAMWCNTs is shown in Figure 4.3(b). Ni catalytic particles are remained at the tips of the asgrown CNTs as can be seen from the SEM image.

\subsubsection{Fabrication of PMMA-CNT matrix- Polishing procedure to expose the tips}

The PMMA solution was prepared by diluting PMMA with the developer solution (Microchem) in a volume ratio of 1:1. The as-grown VA-MWCNTs were then dip coated by poly methyl methacrylate (PMMA) followed by annealing at $\sim 100^{\circ} \mathrm{C}$ for $2-3$ minutes. The schematic diagram of PMMA coated sample is shown in Figure 4.4(a). Samples were then polished to expose the tips of CNTs. We call these PMMA-CNT matrices and they are ready for immobilization of $G O_{x}$. The exposed 
VA-MWCNTs tips were modified following the procedure used by Lin et al [26]. The corresponding SEM image of CNTs dipped in PMMA is shown in Figure 4.4(b).
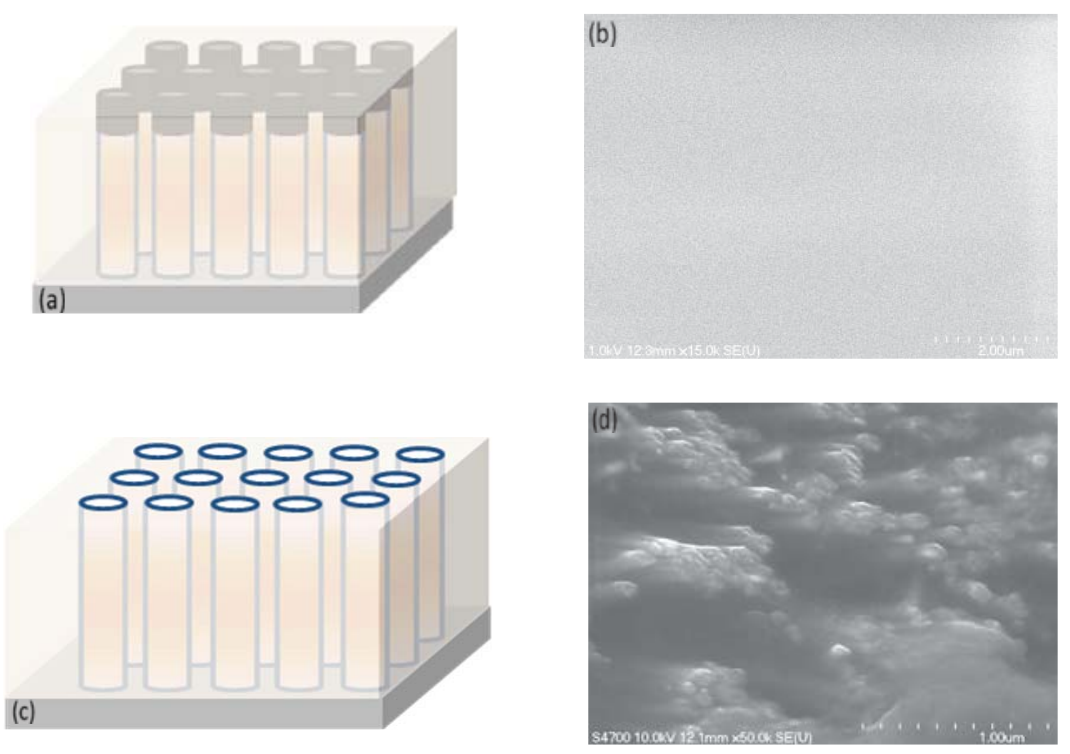

Figure 4.4: (a) Schematic diagram of as grown vertically aligned multi-walled CNTs (VAMWCNTS) dipped in Poly (methyl methacrylate) (PMMA) (b) corresponding SEM image of dipped VA-MWCNTs (c) schematic diagram of polished CNTs to expose the tips of CNTs and (d) Corresponding SEM image of the polished tips

It can be observed that after dipping no CNTs can be seen. The cured samples (Figure 4.4(b)) were then mechanically polished by using fiber-free lapping cloth and a colloidal silica $(0.02 \mu \mathrm{m}$ in diameter) solution to expose the tips of the CNTs. The fabrication scheme of the PMMA-CNTs matrices using PMMA solution is summarized in Figure 4.4. After mechanical polishing the Ni catalyst particles were removed. Figure 4.4(c) shows the schematic of polished CNTs. The corresponding SEM image is shown in Figure 4.4(d). The removals of catalyst particles from the tip 
of CNTs open all the dangling bonds which will be further used to attach the $G O_{x}$ enzyme.

In our method we use covalent attachment approach to immobilize $G O_{x}$ onto tips of CNTs. The advantage of direct anchoring of enzymes by covalent immobilization is that it enables direct electron transfer to the active sites of the enzyme.

\subsubsection{Preparation of biosensor with different working electrode area}

The synthesis of CNTs is same as discussed in above section. In our PECVD chamber we can grow three identical CNT samples (each sample with Si substrate of $1 \mathrm{~cm} \times 1 \mathrm{~cm}$ in size) at a time. The area of the circular CNTs growth region is 0.385 $\mathrm{cm}^{2}$ on Si substrate. For the study of the area effect, a total of eight CNT samples were prepared. The variation in working electrode area is schematically shown in Figure 4.5 .

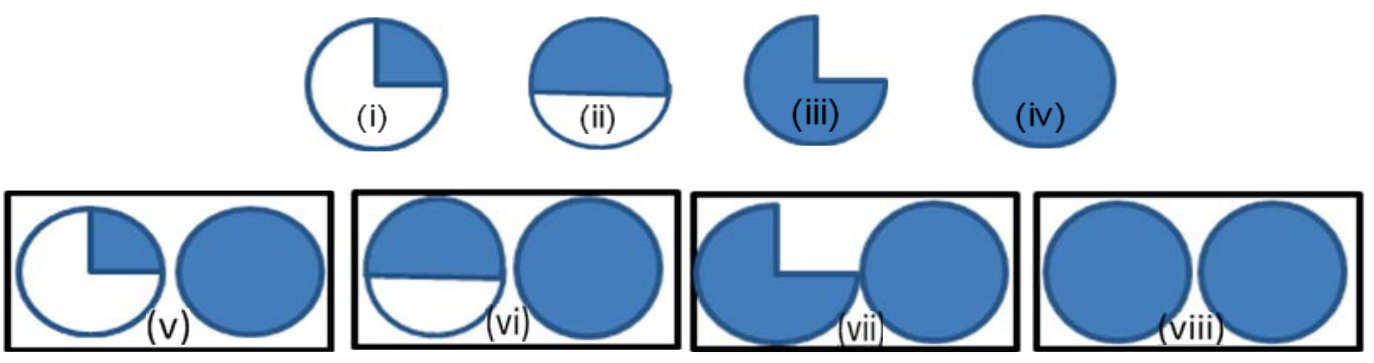

Figure 4.5: The schematic diagram of CNTs with the areas of (i) $0.096 \mathrm{~cm}^{2}$, (ii) $0.193 \mathrm{~cm}^{2}$, (iii) $0.298 \mathrm{~cm}^{2}$, (iv) $0.385 \mathrm{~cm}^{2}$ (v) $0.481 \mathrm{~cm}^{2}$, (vi) $0.578 \mathrm{~cm}^{2}$, (vii) $0.674 \mathrm{~cm}^{2}$ and (viii) $0.77 \mathrm{~cm}^{2}$ 
At first four CNTs samples (Si substrate of $1 \mathrm{~cm} \times 1 \mathrm{~cm}$ in size) were grown within a circular area of $0.385 \mathrm{~cm}^{2}$. After that the samples were scratched according to the required area: for one-fourth area $\left(0.096 \mathrm{~cm}^{2}\right)$, half area $\left(0.193 \mathrm{~cm}^{2}\right)$ and three fourth area $\left(0.0 .298 \mathrm{~cm}^{2}\right)$. Si substrate size of $2 \mathrm{~cm} \mathrm{x} 1 \mathrm{~cm}$ was used to grow CNTs samples for the areas more than $0.385 \mathrm{~cm}^{2}$. Again four CNTs samples were grown using $2 \mathrm{~cm} \times 1 \mathrm{~cm}$ silicon substrates. After that the rest of the deposited CNTs were scratched from the substrate to achieve the required area as depicted in Figure 4.5.

\subsubsection{Functionalization of exposed tips of PMMA-CNT matrices with carboxylic (-COOH) group}

Before immobilization of $G O_{x}$, PMMA-CNT matrices were pretreated electrochemically with $1 \mathrm{M}$ sodium hydroxide $(\mathrm{NaOH})$ at $2.8 \mathrm{~V}$ for $5 \mathrm{~min}$. The modified electrodes were dried for 30 minutes in air. This step was done in order to attach carboxylic group (-COOH) on tips of CNTs. Figure 4.6(a) shows the schematic of the set-up which was used for the functionalization of polished CNTs to attach $-\mathrm{COOH}$ group. Figure 4.6(b) shows the schematic of the $-\mathrm{COOH}$ functionalized CNTs.

Existence of the carboxylic acid group $\left(\sim 1730 \mathrm{~cm}^{-1}\right)$ was confirmed by FTIR spectroscopy [27]. Figure 4.6(c) shows the fourier transform infrared spectroscopy (FTIR) of as-grown sample which shows that there was no carboxylic group present before electrochemical treatment. Once the CNTs tips were opened electrochemical treatment was done by $\mathrm{NaOH}$. 

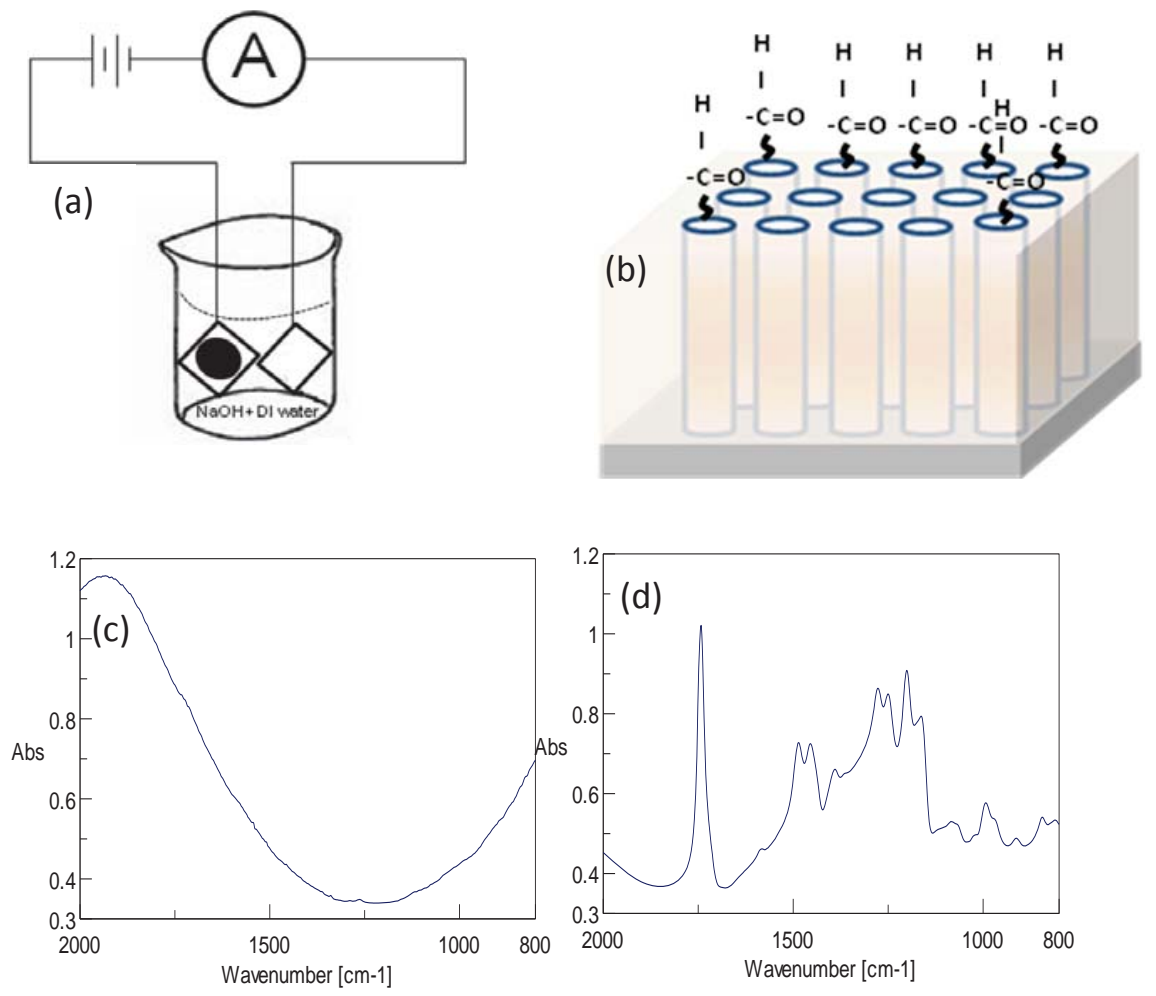

Figure 4.6: Schematic diagram of (a) functionalization of VA-MWCNTs with carboxylic groups (b) functionalization of -COOH on tips of CNTs and (c) FTIR spectra of as grown CNTs and (d) FTIR of-COOH functionalized CNTs.

Figure 4.6(d) shows the FTIR of electrochemically treated CNTs. The bands at $1759 \mathrm{~cm}^{-1}$ and $1160 \mathrm{~cm}^{-1}$ were attributed to the $\mathrm{C}=\mathrm{O}$ and $\mathrm{C}-\mathrm{O}$ stretching vibrations mode of the carboxylic groups respectively. This result confirms the attachment of carboxylic groups on the broken bonds of CNTs tips [27]. 


\subsubsection{Immobilization of $G O_{x}$ on PMMA-CNT matrices}

We use deionized (DI) water for all the experiments described here. The immobilization was done using standard water soluble coupling agent EDC (1-ethyl-33-dimethylaminopropyl carbodiimide) and sulfo-NHS (N-hydroxy-sulfo-succinimide) as reported by Lin et al [26]. At first PMMA-CNT matrices functionalized with $\mathrm{COOH}$ groups were immersed in $10 \mathrm{ml}$ of EDC aqueous solution $(10 \mathrm{mg} / \mathrm{ml})$. Next, $300 \mathrm{mg}$ of sulfo-NHS was added to the above solution with stirring. Chemical reactions were allowed to occur at room temperature for 3 hours. Finally the above samples were washed in DI water and immersed in degassed $G O_{x}$ solution $(2 \mathrm{mg} / \mathrm{ml})$ prepared in $20 \mathrm{ml}$ of $0.1 \mathrm{M}$ phosphate buffer solution $\left(\mathrm{pH}\right.$ 7.2). $G O_{x}$ immobilization was allowed to occur at room temperature for 3 hours. The glucose biosensors based on PMMA-CNT matrices were stored at $4^{0} \mathrm{C}$ in PBS solution when not in use. The possible reactions involved are given in the scheme in Figure 4.7.

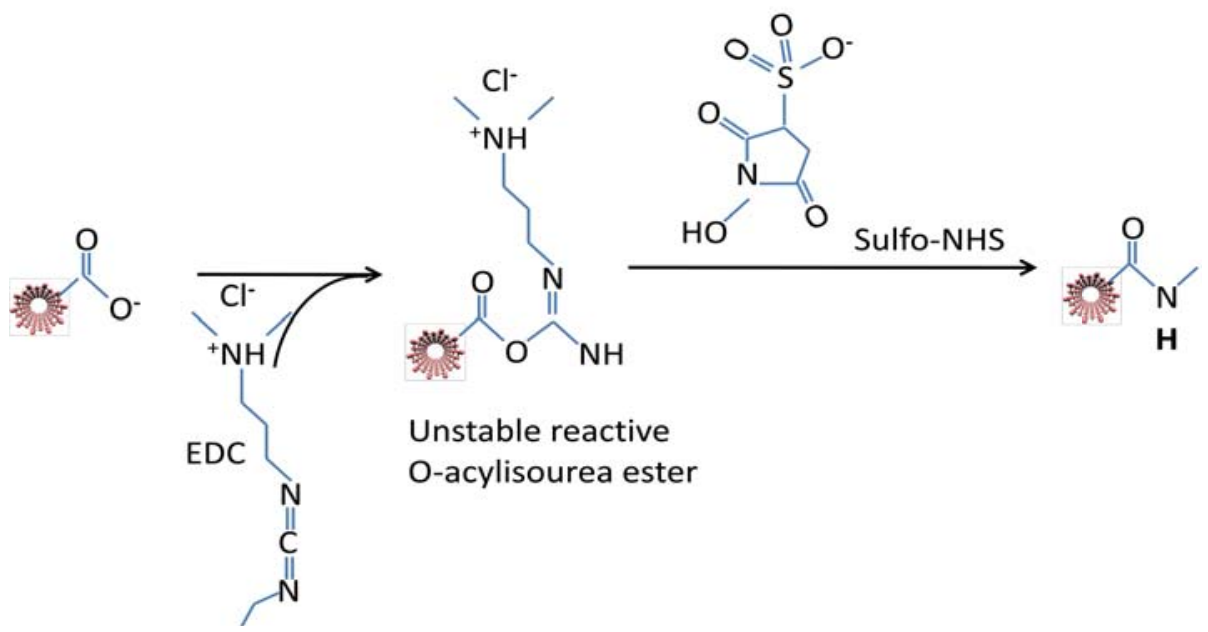

Figure 4.7: Reaction involving EDC and Sulfo-NHS 


\subsubsection{Assembling glucose biosensor and its measurement}

The glucose biosensor was incorporated into a three-electrode electrochemical cell (EC cell) as the working electrode as shown in Figure 4.8(a). Phosphate buffer solution (PBS, pH 7.2) was used as the solvent.
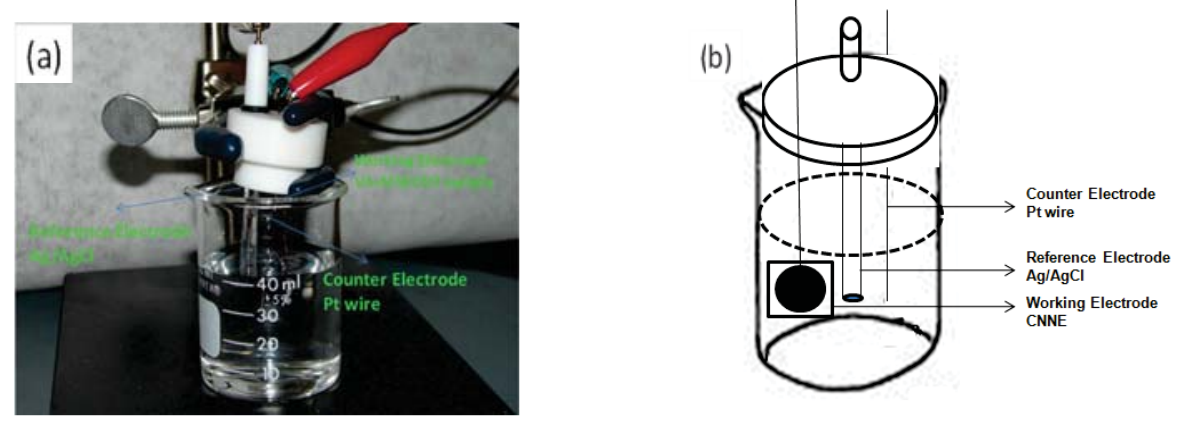

Figure 4.8: Picture of (a) electrochemical cell (EC cell) and (b) schematic diagram showing three electrodes

A gold target (and platinum wire) was used as the counter electrode with an $\mathrm{Ag} / \mathrm{AgCl}$ reference electrode. Amperometric response was obtained using a computer controlled potentiostat $\mathrm{CHI}$ 660C. The EC cell allowed to stabilize for at least $500 \mathrm{~s}$ before adding the glucose solution (analyte) to the system for sensing measurement / current changes induced by glucose $-G O_{x}$ binding. Figure 4.8(b) shows the schematic diagram of three electrodes in EC cell. 


\subsection{Results and Discussion}

\subsubsection{Cyclic Voltammetry (CV) measurements}

CV of PMMA-CNT matrixes without $G O_{x}$ are flat and show no redox peaks. This indicates that there is no leakage current and the PMMA-CNT matrices are sealed completely. Next we performed CV of $G O_{x}$ immobilized on PMMA-CNT matrices as shown in Figure 4.9(a). CV was performed in deaerated (purging with nitrogen gas for 10min) PBS (pH 7.2). Direct electron transfer (DET) behavior of $G O_{x}$ immobilized on PMMA-CNTs matrices is obtained. Figure 4.9(a) shows the CV of $G O_{x}$-PMMA-CNT electrodes in PBS ( $\mathrm{pH}$ 7.2). $\mathrm{CV}$ curve for $G O_{x}$-PMMA-CNTs biosensor shows a pair of well defined redox peaks with the anodic and cathodic peak potentials at $-316 \mathrm{mV}$ and $-311 \mathrm{mV}$ respectively at a scan rate of $100 \mathrm{mV} / \mathrm{sec}$. The pair of redox peaks in Figure 4.9(a) corresponds to DET reaction of $G O_{x}$ as follows:

Reduction: $G O_{x}(\mathrm{FAD})+2 \mathrm{e}^{-}+2 \mathrm{H}^{+} \rightarrow G O_{x}\left(\mathrm{FADH}_{2}\right)$

Oxidation: $G O_{x}\left(\mathrm{FADH}_{2}\right) \rightarrow G O_{x}(\mathrm{FAD})+2 \mathrm{e}^{-}+2 \mathrm{H}^{+}$

In the forward scan (from $\mathrm{A}$ to $\mathrm{B}$ to $\mathrm{C}$ in Figure 4.9(a)) $G O_{x}(\mathrm{FAD})$ is reduced to $G O_{x}\left(\mathrm{FADH}_{2}\right)$. This results in reduction peak or cathodic current peak at $\mathrm{B}(-$ $311 \mathrm{mV}$ ). In the reverse scan (from $\mathrm{C}$ to $\mathrm{D}$ to $\mathrm{A}$ in Figure 4.9(a)) $G O_{x}\left(\mathrm{FADH}_{2}\right)$ is oxidized back to $G O_{x}(\mathrm{FAD})$. This results in oxidation peak or anodic current peak at D $(-316 \mathrm{mV})$. This confirms that $G O_{x}$ immobilized on VA-MWCNTs nano-electrode surface is electro-active and is stable. 
Figure 4.9(b) shows the $\mathrm{CV}$ of $G O_{x}$-PMMA-CNT with glucose addition. After the addition of $12 \mathrm{ml}$ of $0.22 \mathrm{mM}$ glucose solution to $218 \mathrm{ml}$ of PBS the shape of the $\mathrm{CV}$ of $G O_{x}$-PMMA-CNT electrodes changes significantly and is characterized by a large anodic current peak at $-195 \mathrm{mV}$ without any cathodic peak current.
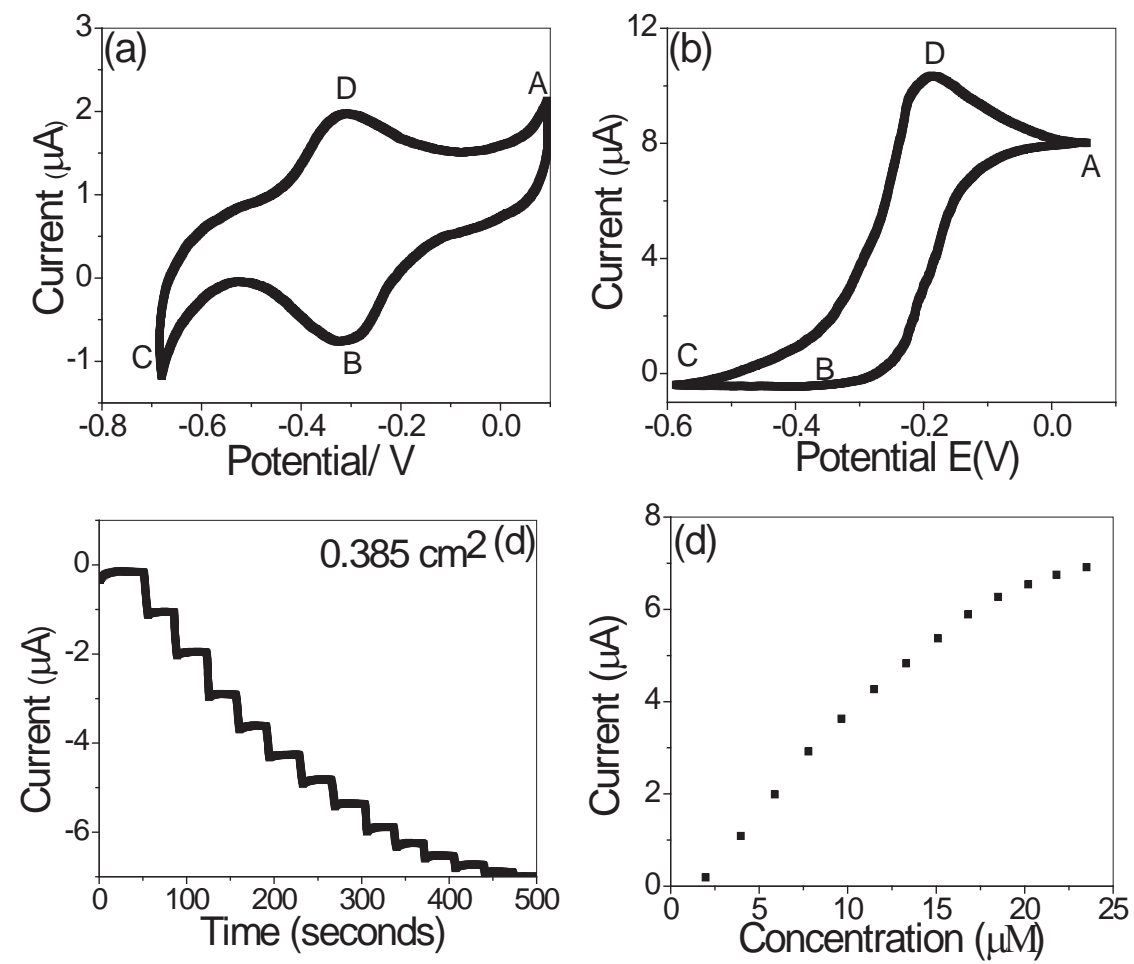

Figure 4.9: Cyclic voltammogram of (a) GO $O_{x}$-PMMA-CNTs biosensor and (b) GO-PMMACNTs biosensor after glucose addition respectively at scan rate of $100 \mathrm{mV} / \mathrm{s}$. (c) Amperometric (current-time) response of $G O_{x}-P M M A-C N T s$ matrices with successive stepwise addition of $2 \mathrm{ml}$ of $0.22 \mathrm{mM}$ glucose solution to $218 \mathrm{ml} P B S$ (pH 7.2) (d) Calibration curve of Current response vs. Glucose concentration. 
The increase of glucose concentration leads to the enhancement of the anodic current. The electro-catalytic reactions occurring at the working electrode can be written as follows:

Reduction: $G O_{x}(\mathrm{FAD})+$ glucose $\rightarrow G O_{x}\left(\mathrm{FADH}_{2}\right)+$ gluconolactone (from A to $\mathrm{B}$ to $\mathrm{C})$

Oxidation: $G O_{x}\left(\mathrm{FADH}_{2}\right) \rightarrow G O_{x}(\mathrm{FAD})+2 \mathrm{e}^{-}+2 \mathrm{H}^{+}$(from $\mathrm{C}$ to $\mathrm{D}$ to $\mathrm{A}$ )

Therefore, $G O_{x}$ immobilized on PMMA-CNTs can be used as biosensors for glucose sensing. As shown in equations (1)-(4), our BFCs involved three possible reactions. Equations (1), (2) and (4) are electrochemical reactions and equation (3) is an enzymatic reaction. An enzymatic reaction does not produce any peak which can be observed in CV graph. When the glucose is added to the solution only reactions (1) and (3) are competing with each other. Since the enzymatic reaction is much faster and dominated, we observe that $G O_{x}(\mathrm{FAD})$ is reduced in $G O_{x}\left(\mathrm{FADH}_{2}\right)$ and glucose is oxidized to gluconolactone. This reaction (equation 3) produced $G O_{x}\left(\mathrm{FADH}_{2}\right)$ in large amount. When the reduced form of $G O_{x}\left(\mathrm{FADH}_{2}\right)$ is oxidized back to $G O_{x}$ (FAD) we observe (equation 4) the reduction peak decreases but oxidation peak increases.

Figure 4.9(c) shows the amperometric response of $G O_{x}$-PMMA-CNT biosensor to the successive addition of $2 \mathrm{ml}$ of $0.22 \mathrm{mM}$ glucose solution to $218 \mathrm{ml}$ PBS at $-0.4 \mathrm{~V}$ and it shows the highest electro-catalytic activity towards glucose. Immediately after the addition of glucose the current response decreases and reaches a 
steady state within $\sim 30$ seconds suggest that the electrode responds rapidly to the change of the substrate concentration. As shown in Figure 4.9(d), a linear response of the biosensor can be obtained for glucose concentration up to $\sim 20 \mu \mathrm{M}$. The sensitivity of the sensors (gradient of linear region of Figure 4.9(d) sensor area) was found to be $1.0342 \mu \mathrm{A} / \mu \mathrm{M} / \mathrm{cm}^{2}$ for a sensor of area $0.385 \mathrm{~cm}^{2}$.

To test whether biosensor can be used for continuous monitoring of glucose, I studied the continuous amperometric current response of glucose biosensor with one step glucose addition. For this, first the biosensor was stabilized/equilibrated in PBS ( $\mathrm{pH}$ 7.2) for few hours and then the $20 \mathrm{ml}$ of $0.22 \mathrm{mM}$ glucose solution was added to the $218 \mathrm{ml}$ of PBS and current response was recorded continuously for $\sim 22$ hours at $0.4 \mathrm{~V}$. Figure 4.10(a) shows the amperometric response of the single step addition of glucose to $G O_{x}$-PMMA-CNTs biosensor.
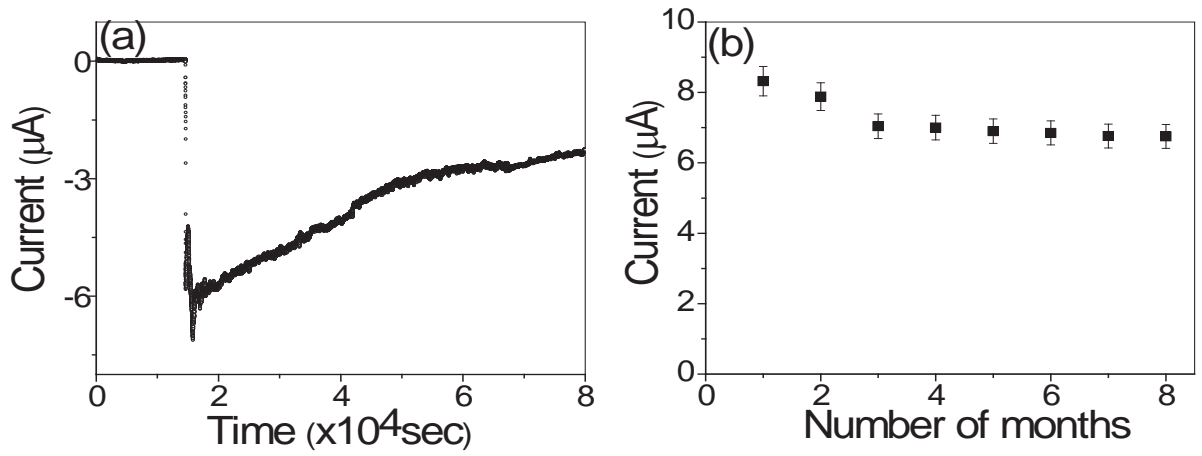

Figure 4.10: (a) i-t response of $G O_{x}-P M M A-C N T$ biosensor with single step glucose addition for $\sim 22$ hours. Only partial recovery $(\sim 65 \%)$ is observed and (b) Stability curve of $G O_{x^{-}}$ PMMA-CNT biosensors. Stability of enzyme was monitored at regular intervals of 5-6 days. 
Clearly it is observed from Figure 4.10(a) that even after long period of time the biosensor partially recovers its activity ( $\sim 65 \%$ in 22 hours). Initial order of magnitude calculations from area under the curve indicate that the rate of glucose consumption for the biosensor is 0.0104 nanomol/s. Details of this calculation are shown in Appendix A-1.

Our $G O_{x}$-PMMA-CNT glucose biosensor shows stability and maintains the activity of the $G O_{x}$ enzyme for more than nearly eight months. The stability of the $G O_{x}$-PMMA-CNT biosensor was investigated under the storage condition, PBS, $\mathrm{pH}$ $7.2,4^{0} \mathrm{C}$, using the same glucose concentration $(20 \mathrm{ml}$ of $0.22 \mathrm{mM}$ glucose solution was added to the $218 \mathrm{ml}$ of PBS) and the result of variation in amperometric response at $-0.4 \mathrm{~V}$ is shown in Figure 4.10(b). As can be seen from the graph after $\sim 8$ months the response current is still retained at $70 \%$ of the initial value. The decrease of the current response can be due to the result of the decrease in the enzyme activity or denaturation of enzyme (unfolding) during storage. Compared to the reported glucose biosensors based on CNTs paste electrodes [20] which could maintain stability within 3 to 120 days, the usability of our biosensors is much longer $\sim 8$ months. Based on our result shown in Figure 4.10(b) we can conclude that the opened tip PMMA-CNT matrices of the $G O_{x}$-PMMA-CNT electrode is biocompatible with the immobilized enzyme and is able to maintain the activity of the enzyme $G O_{x}$ for a long period of time. 


\subsubsection{Effect of electrode area on performance of biosensors}

As emphasized in previous section that sensitive detection of biomolecules is very important in numerous biomedical applications. These applications of biosensors require miniaturization from micro-to-nano scale sensors for all the implantable devices. Miniaturization is also very important in fields such as point of care biodiagnostics, lab on a chip application for sensitive detection of small concentration of biomolecules with improved sensitivity and biocompatibility. The use of functionalized PMMA-CNT matrices (act as working electrode) can fulfill this demand due to its small size and providing enough surface area for enzyme immobilization.

In this section the effects of working electrode area on performance of glucose biosensor are discussed. The different working electrode areas used for this study were $0.096 \mathrm{~cm}^{2}, 0.193 \mathrm{~cm}^{2}, 0.289 \mathrm{~cm}^{2}, 0.385 \mathrm{~cm}^{2}, 0.481 \mathrm{~cm}^{2}, 0.578 \mathrm{~cm}^{2}, 0.674 \mathrm{~cm}^{2}$ and $0.77 \mathrm{~cm}^{2}$ as detailed in section 4.3.3.

All the experiments were performed using our CHI 660 electrochemical workstation and the three-electrode setup with $\mathrm{Ag} / \mathrm{AgCl}$ reference electrode. A gold

plate (area $\sim 6.00 \mathrm{~cm}^{2}$ ) was used as the counter electrode and $G O_{x}$ immobilized on PMMA-CNT were used as working electrode as discussed in the earlier section. 

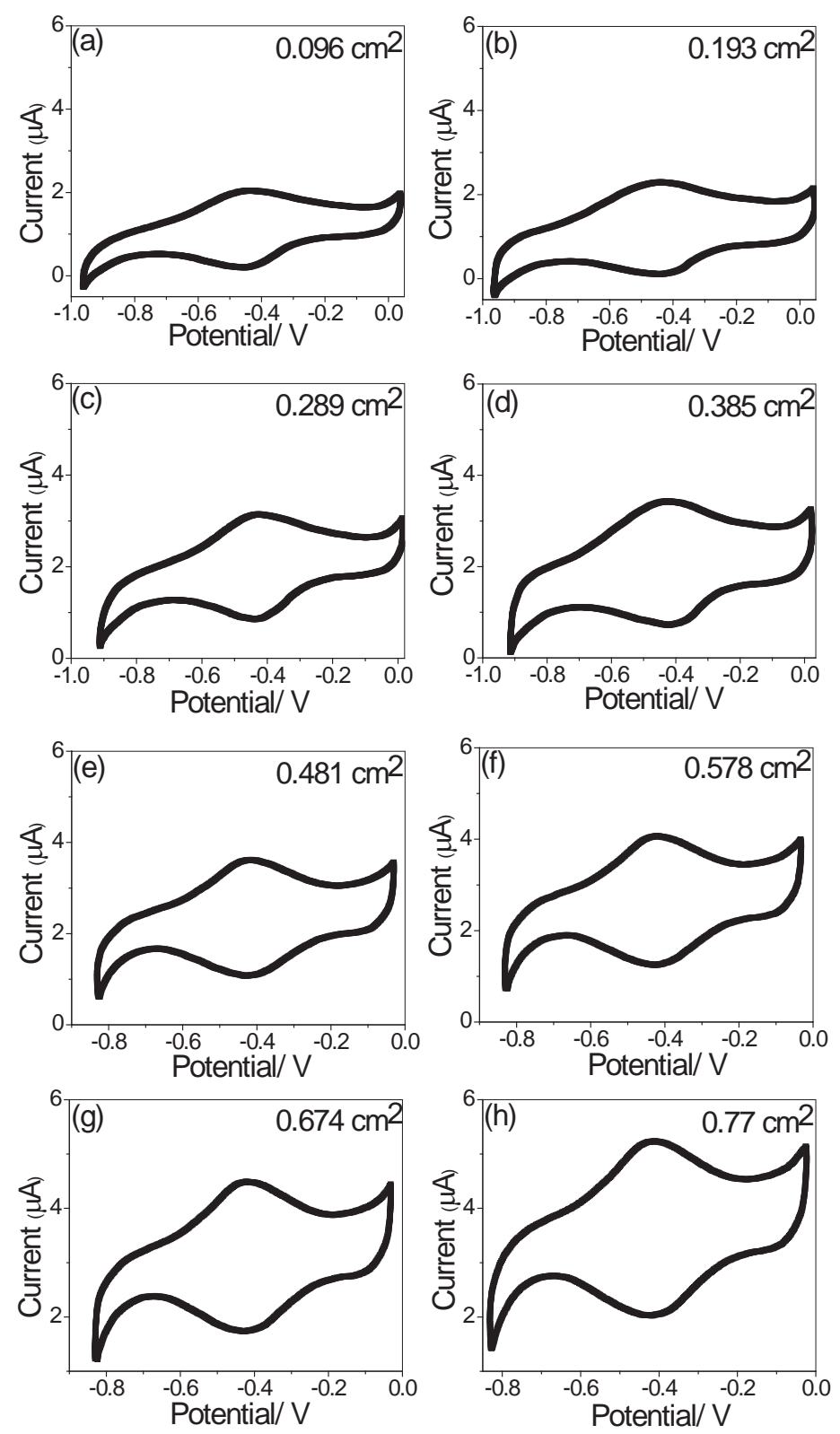

Figure 4.11: Cyclic voltammogram of (a-h) GO $O_{x}-P M M A-C N T$ biosensor show two symmetrical peaks with anodic and cathodic peaks for areas of $0.096 \mathrm{~cm}^{2}, 0.193 \mathrm{~cm}^{2}, 0.289$ $\mathrm{cm}^{2}, 0.385 \mathrm{~cm}^{2}, 0.481 \mathrm{~cm}^{2}, 0.578 \mathrm{~cm}^{2}, 0.674 \mathrm{~cm}^{2}$ and $0.77 \mathrm{~cm}^{2}$ respectively at scan rate of $100 \mathrm{mV} / \mathrm{s}$. 

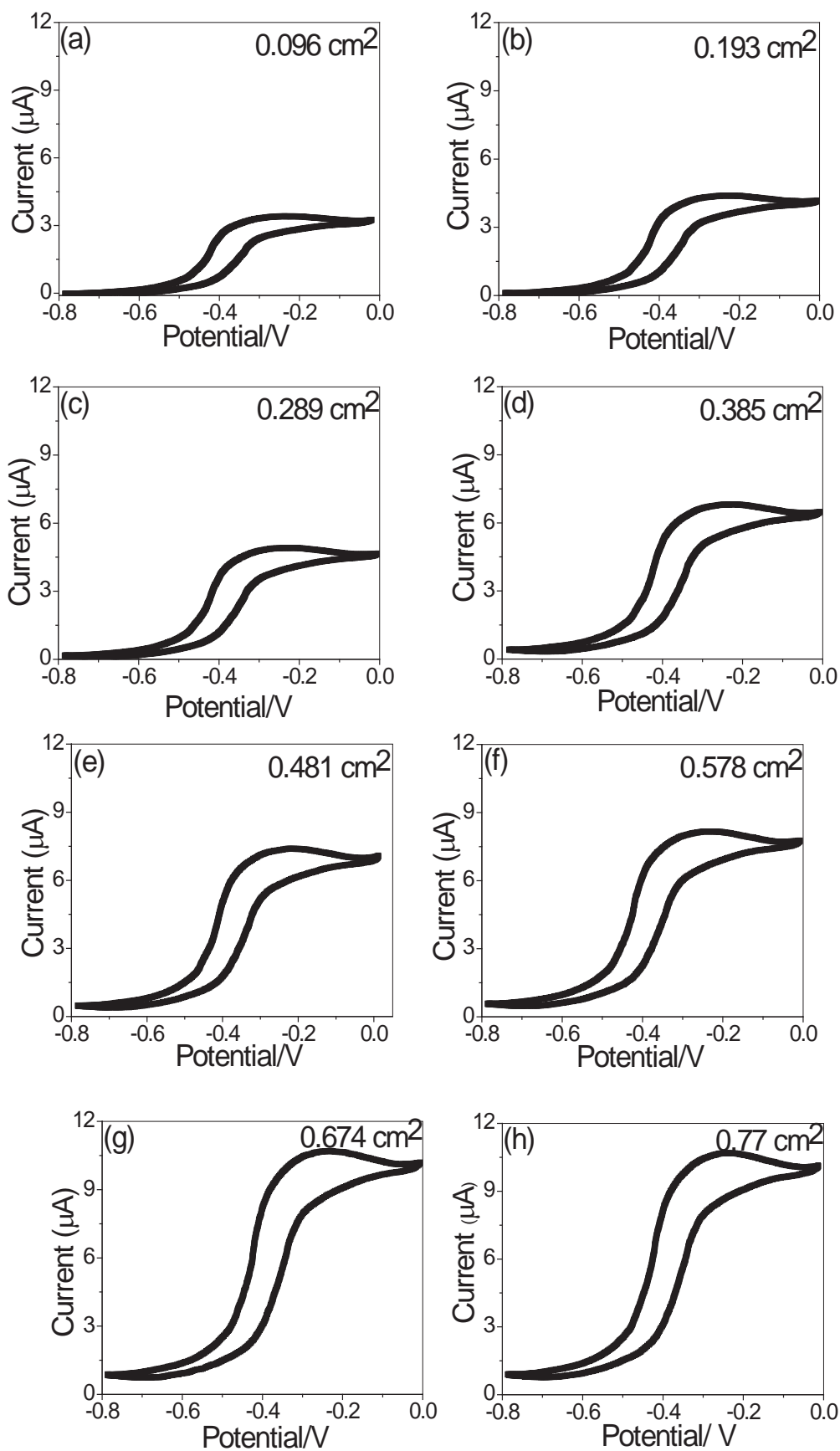

Figure 4.12: Cyclic voltammogram of (a)-(h) GO $O_{x}-P M M A-C N T$ biosensor after adding glucose solution for areas of $0.096 \mathrm{~cm}^{2}, 0.193 \mathrm{~cm}^{2}, 0.289 \mathrm{~cm}^{2}, 0.385 \mathrm{~cm}^{2}, 0.481 \mathrm{~cm}^{2}, 0.578 \mathrm{~cm}^{2}$, $0.674 \mathrm{~cm}^{2}$ and $0.77 \mathrm{~cm}^{2}$ respectively at scan rate of $100 \mathrm{mV} / \mathrm{s}$. 

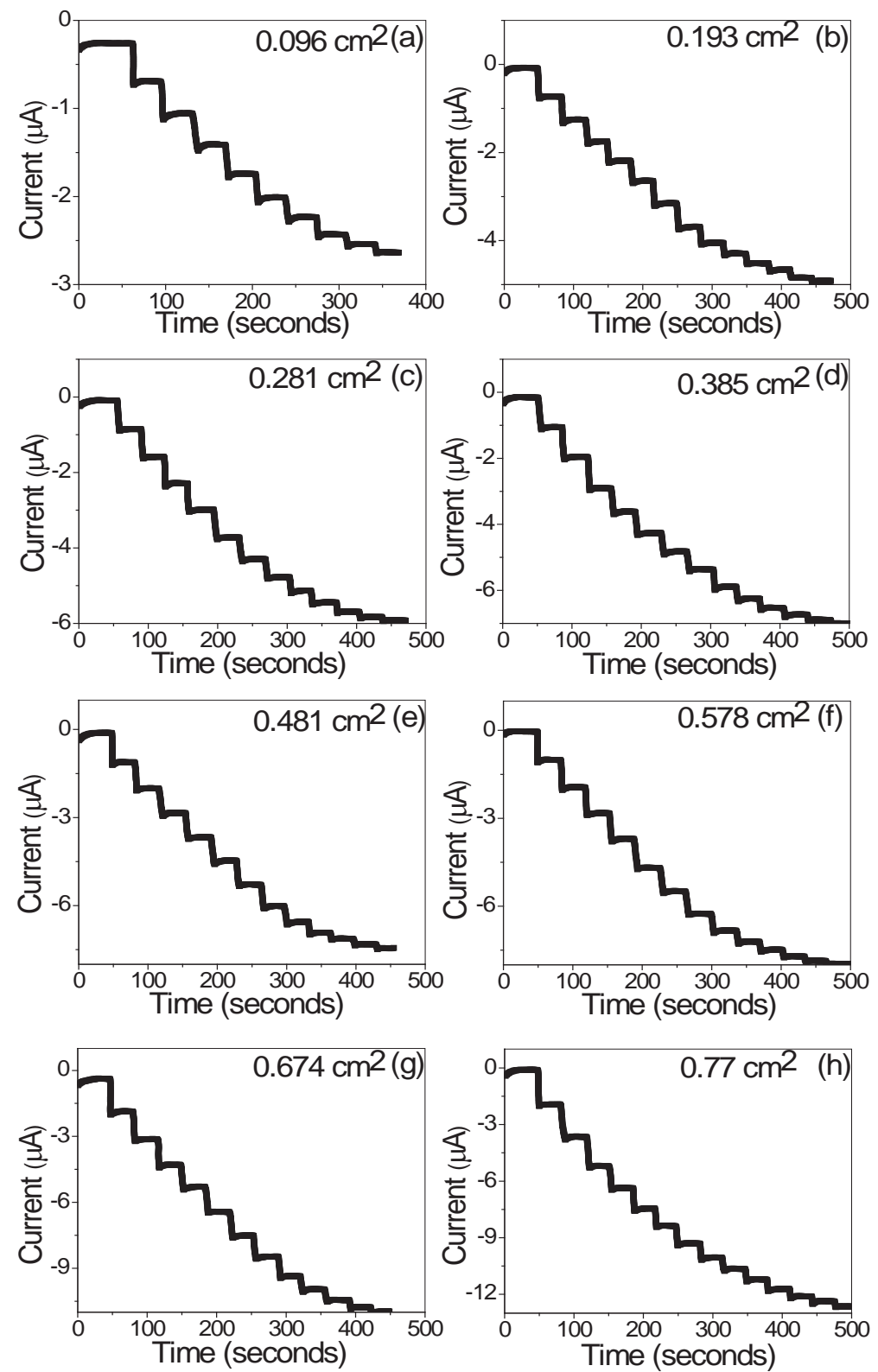

Figure 4.13: Amperometric response (a)-(h) (i-t) of $G O_{x}-P M M A-C N T$ biosensor with successive addition of $1 \mathrm{mM}$ glucose in PBS ( $\mathrm{pH}$ 7.2) for areas $0.096 \mathrm{~cm}^{2}, 0.193 \mathrm{~cm}^{2}, 0.289$ $\mathrm{cm}^{2}, 0.385 \mathrm{~cm}^{2} 0.481 \mathrm{~cm}^{2}, 0.578 \mathrm{~cm}^{2}, 0.674 \mathrm{~cm}^{2}$ and $0.77 \mathrm{~cm}^{2}$ respectively. 
Figures 4.11(a)-(h) shows the $\mathrm{CV}$ of $G O_{x}$ immobilized PMMA-CNT with different areas of $0.096 \mathrm{~cm}^{2}, 0.193 \mathrm{~cm}^{2}, 0.289 \mathrm{~cm}^{2}, 0.385 \mathrm{~cm}^{2}, 0.481 \mathrm{~cm}^{2}, 0.578 \mathrm{~cm}^{2}$, $0.674 \mathrm{~cm}^{2}$ and $0.77 \mathrm{~cm}^{2}$, respectively without adding glucose. For all the cases a pair of two symmetrical peaks can be clearly seen on these $G O_{x}$ immobilized PMMA-CNT matrices as already described for Figure 4.9(a).

Figure 4.12 (a)-(h) show $\mathrm{CV}$ of $G O_{x}$-PMMA-CNT biosensors with areas of $0.096 \mathrm{~cm}^{2}, 0.193 \mathrm{~cm}^{2}, 0.289 \mathrm{~cm}^{2}, 0.385 \mathrm{~cm}^{2}, 0.481 \mathrm{~cm}^{2}, 0.578 \mathrm{~cm}^{2}, 0.674 \mathrm{~cm}^{2}$ and $0.77 \mathrm{~cm}^{2}$ respectively, after addition of $12 \mathrm{ml}(0.22 \mathrm{mM})$ glucose solution in $218 \mathrm{ml}$ of PBS. Anodic peak increases whereas the cathodic peak decreases for all the areas which are similar to the behavior described previously. As shown in Figures 4.12(a)(h), the areas under the anodic peaks increase as the device area increases. This is understandable since as area increases more number of $G O_{x}$ enzyme immobilized sites contribute to the current.

Figures 4.13(a)-(h) shows amperometric (i-t) response of $G O_{x}$-PMMA-CNT biosensors with successive addition of $2 \mathrm{ml}$ glucose solution $(0.22 \mathrm{mM})$ in $218 \mathrm{ml} \mathrm{PBS}$ $(\mathrm{pH} 7.2)$ at $\quad-0.4 \mathrm{~V}$ for device areas of $0.096 \mathrm{~cm}^{2}, 0.193 \mathrm{~cm}^{2}, 0.289 \mathrm{~cm}^{2}, 0.385 \mathrm{~cm}^{2}$ and $0.481 \mathrm{~cm}^{2}, 0.578 \mathrm{~cm}^{2}, 0.674 \mathrm{~cm}^{2}$ and $0.77 \mathrm{~cm}^{2}$, respectively. For each addition droplet of glucose solution, a sharp decrease in current was observed.

The response reached steady state value in $\sim 30 \mathrm{sec}$. As the cumulative glucose concentration in the PBS was increased with time, the current responses decreased in magnitude. As shown, at higher glucose concentration, the step size of the current 
response is decreasing and approaching saturation. In addition, the glucose concentration for such saturation to occur is higher for sensors with larger areas (those in $\mathrm{a}$, and $\mathrm{b}$ are higher than those in $\mathrm{g}$ and $\mathrm{h}$ ).

Figure 4.14 shows the combined calibration curve of cumulative current response vs. cumulative glucose concentration for different electrode areas. It is observed clearly that as electrode area increases the linear range of current response also increases and it saturates at a higher glucose concentration. This behavior is expected since as area increases more number of $G O_{x}$ enzyme immobilized sites are available to sense glucose and contribute to the current.

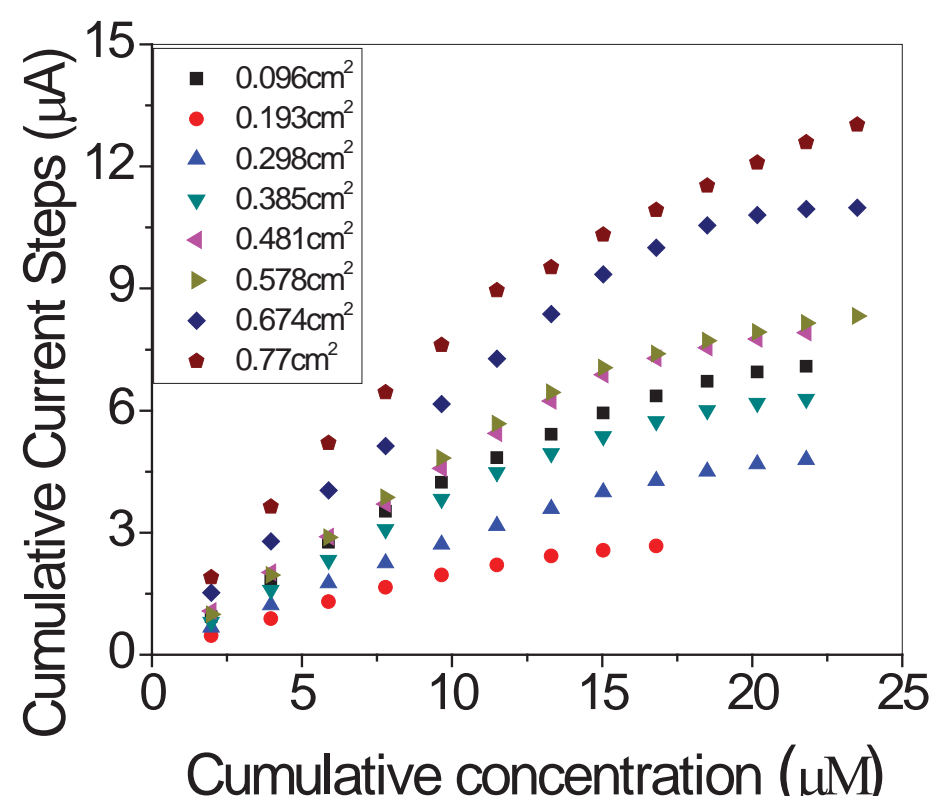

Figure 4.14: Calibration curve of cumulative current response vs. cumulative glucose concentration for $G O_{x}-P M M A-C N T$ biosensor for areas $0.096 \mathrm{~cm}^{2}, 0.193 \mathrm{~cm}^{2}, 0.289 \mathrm{~cm}^{2}$, $0.385 \mathrm{~cm}^{2} 0.481 \mathrm{~cm}^{2}, 0.578 \mathrm{~cm}^{2}, 0.674 \mathrm{~cm}^{2}$ and $0.77 \mathrm{~cm}^{2}$ respectively. 
Table 4.1 summarizes the variation of slope and sensitivity values with different areas. From Table 4.1 we can observe that the slope of current response vs. glucose concentration increases with the increase of sensor area. This can be understood because the number of electroactive $G O_{x}$ sites is increased and available for the immobilization of $G O_{x}$. Hence we obtain a faster response (higher currentconcentration gradient) for sensors with larger area.

Table 4.1: Variation of biosensor parameters with different electrode areas

\begin{tabular}{|c|c|c|}
\hline $\begin{array}{l}\text { Area } \\
\left(\mathrm{cm}^{2}\right)\end{array}$ & $\begin{array}{c}\text { Slope } \\
(\mu \mathrm{A} / \mu \mathrm{M})\end{array}$ & $\begin{array}{l}\text { Sensitivity } \\
\left(\mu \mathrm{A} / \mu \mathrm{M} / \mathrm{cm}^{2}\right)\end{array}$ \\
\hline 0.096 & 0.1825 & 1.901 \\
\hline 0.193 & 0.2598 & 1.3461 \\
\hline 0.298 & 0.3565 & 1.1963 \\
\hline 0.385 & 0.3627 & 0.9421 \\
\hline 0.481 & 0.4297 & 0.8935 \\
\hline 0.578 & 0.4590 & 0.7941 \\
\hline 0.674 & 0.57181 & 0.8484 \\
\hline 0.77 & 0.6524 & 0.8473 \\
\hline
\end{tabular}


Limit of detection was calculated by fitting the linear region of a current vs. concentration curve. Linear region was fitted with a straight line and limit of detection was determined. Fitted calibration curves are given in Appendix-B, Figure B.1 and B.2. For all the areas the limit of detection is close to zero.

Variation of sensitivity versus area is given in Table 4.1. Sensitivity slowly decreases with increase in area. Although range of current for linear response increases as area increases but increase in current lags behind increase of area. In other words number of electroactive $G O_{x}$ sites does not increase as much as area increases. Therefore, even if the current increases because of increase in area it does not overcome the area effect as area is doubled each time. Since area is inversely proportional therefore the overall combined effect of both the factors is that sensitivity decreases as area increases.

In fact, we found that the counter electrode area will affect the measurement of current response discussed so far. In another set of experiments, Pt wire was used as counter electrode for the study of sensor area effect. We found that the current response vs. glucose concentration curve are almost similar for sensors with areas $0.385 \mathrm{~cm}^{2}, 0.481 \mathrm{~cm}^{2}, 0.578 \mathrm{~cm}^{2}, 0.674 \mathrm{~cm}^{2}$ and $0.77 \mathrm{~cm}^{2}$ (Appendix B, Figure B.3, B.4 and B.5 gives CV data and calibration curves).

These can be explained if we consider the area of our Pt wire counter electrode dipped in the buffer solution $\left(0.4374 \mathrm{~cm}^{2}\right)$. Electrons between the sensor and the Pt wire will conduct through the electrolyte/PBS to complete the circuit. Since the 
motion of ions in the PBS buffer solution near the Pt counter electrode is a diffusion limited process, the amount of electrons/ions involve in each complete circuit flow will depends on the area of the Pt wire. For sensors with area less than the area of the Pt wire, the whole current from the sensor can be picked up by the Pt wire. This is not the case for sensors with larger areas as the Pt wire can only pick up a portion of electrons from the sensors. This is especially true as the actual Pt area facing the sensor is less than $0.4374 \mathrm{~cm}^{2}$.

\subsection{Conclusions}

The use of PMMA-CNTs matrices in glucose biosensor was very useful in fabricating effective biosensors. This chapter presents three work altogether:

(1) Glucose biosensor based on $G O_{x}$ immobilized on PMMA-CNTs matrices were fabricated for glucose detection. This biosensor exhibits a strong response to glucose. It also shows a high stability of $\sim 70 \%$ of the initial activity after several months in proper storage. Opened tip PMMA-CNT matrices of the $G O_{x}$-PMMA-CNTs electrode are biocompatible with the immobilized enzyme and are able to maintain the activity of the enzyme $G O_{x}$ for a long period of time.

(2) The next result is effect of electrode area on BFCs performance. As electrode area increases the linear range of current response also increases and it saturates at a higher glucose concentration. Limit of detection is close to zero 
for all the areas. We found that the sensitivity of our biosensor decreases with increase in area. The reason behind this is the inverse dependence of sensitivity on area of the electrode.

\subsection{Future Work on Glucose Biosensors}

Glucose biosensor based on $G O_{x}$ immobilized on PMMA-CNTs matrices can be further improved by optimizing the testing conditions such as $\mathrm{pH}$ of the electrolyte, temperature of testing. Also testing on real blood sample and comparing it with existing technologies can help to understand its performance better. We can also employ better immobilization techniques to increase the number of electroactive enzymes as we increase the area of the electrodes. We can also use different and better techniques to coat VA-MWCNTs with nanoparticles uniformly with different sizes to optimize and enhance the properties of these biosensors.

\subsection{References}

1) D. R. Thévenot, K. Toth, R. A. Durst, G. S. Wilson. Electrochemical biosensors: Recommended definitions and classifications. Anal. Lett. 2001, 34, 635.

2) M. C. Tran. Biosensors, Chapman \& Hall Inc., 1993, ISBN 0412481901

3) D. Müller. Studies of a new enzyme: glucose oxidase, Biochem. Z. 1928, 199, 136. 
4) J. Raba, H. A. Mottola, Glucose oxidase as an analytical reagent, Critical Reviews in Analytical Chemistry, 1995, 25, 1.

5) R. Wilson, A. P. F. Turner. Glucose oxidase-an ideal enzyme, Biosens. Bioelectron. 1992, 7, 165.

6) B. E. P. Swoboda, V. Massey. Purification and properties of the glucose oxidase from aspergillus niger, The Journal of Biological Chemistry, 1965, $240,2209$.

7) J. A. Cracknell, K. A. Vincent, F. A. Armstrong. Enzymes as working or inspirational electrocatalysts for fuel cells and electrolysis, Chem. Rev. 2008, $108,2439$.

8) National Diabetes Fact Sheet, Department of health and human services, centers for disease control and prevention, 2007.

9) S. Wild, G. Roglic, A. Green, R. Sicree, H. Kind. Global prevalence of diabetes: estimates for the year 2000 and projections for 2030, Diabetes care, $2004,27,1047$.

10) M. Baxendale. The physics and applications of carbon nanotubes J. Mater. Sci.: Mater. Electron, 2003, 14, 657.

11) W. R. Yang, P. Thordarson, J. J. Gooding, S. P. Ringer, F. Braet. Carbon nanotubes for biological and biomedical applications Nanotechnology, 2007, 18,412001 . 
12) S. N. Kim, J. F. Rusling, F. Papadimitrakopoulos. Carbon nanotubes for electronic and electrochemical detection of biomolecules Adv. Mater, 2007, 19,3214 .

13) Y. H. Yun, Z. Y. Dong, V. Shanov,W. R. Heineman, H. B. Halsall, A. Bhattacharya, L. Conforti, R. K. Narayan, W. S. Ball, M. J. Schulz. Nanotube electrodes and biosensors, Nano Today, 2007, 2, 30.

14) Y. H. Lin, W. Yantasee, J. Wang. Carbon nanotubes (CNTs) for the development of electrochemical biosensors. Front. Biosci 2005, 10, 492.

15) J. Wang. Carbon-nanotube based electrochemical biosensors: A review, Electroanalysis, 2005, 17, 7.

16) P. Xinyu, H. Dongmei, L. Shenglian, C. Qingyun. An amperometric glucose biosensor fabricated with $\mathrm{Pt}$ nanoparticle-decorated carbon nanotubes $/ \mathrm{TiO}_{2}$ nanotube arrays composite Sensors and Actuators B, 2009, 137, 134.

17) B. Pérez, M. Pumera, M. D. Valle, A. Merkoçi, S. Alegret. Glucose biosensor based on carbon nanotube epoxy composites, Journal of Nanoscience and Nanotenhnology, 2005, 5, 1694.

18) B. Pérez, M. D. Valle, S. Alegret, A. Merkoçi. Carbon nanofiber vs. carbon microparticles as modifiers of glassy carbon and gold electrodes applied in electrochemical sensing of NADH, Talanta, 2007, 74, 398. 
19) J. Jinyan, G. Wenjun, S. Minghao, L. Yongquan, L. Hong. Carbon nanotubes based glucose needle-type biosensor, Sensors, 2008, 8, 1712.

20) K. Balasubramanian, M. Burghard. Biosensors based on carbon nanotubes, Anal Bioanal Chem, 2006, 385, 452.

21) J. Wang, M. Musameh, Enzyme-dispersed carbon-nanotube electrodes: a needle microsensor for monitoring glucose, Analyst, 2003, 128, 1382.

22) F. Battaglini, P. N. Bartlett, J. H. Wang. Covalent attachment of osmium complexes to glucose oxidase and the application of the resulting modified enzyme in an enzyme switch responsive to glucose, Anal. Chem., 2000, 72, 502.

23) H. J. Wang, C. M. Zhou, F. Peng, H. Yu. An enhanced glucose biosensor modified by Pt/sulfonated-MWCNTs with layer by layer technique, Int. J. Electrochem. Sci., 2007, 2, 508.

24) S. Sotiropoulo, N. A. Chaniotakis. Stabilization of enzymes in nanoporous materials for biosensor applications, Anal Bioanal. Chem., 2003, 375, 103.

25) S. Hrapovic, Y. Liu, K. B. Male, J. H. T. Luong. Electrochemical biosensing platforms using platinum nanoparticles and carbon nanotubes, Anal. Chem. 2004, 76, 1083.

26) Y. Lin, F. Lu, Y. Tu, Z. Ren. Glucose biosensors based on carbon nanotube nanoelectrode ensembles, Nanoletters, 2004, 4, 191. 
27) D. B. Mawhinney, V. Maumenko, A. Kuznetsova, J. T. Yates. Infrared spectral evidence for the etching of carbon nanotubes: Ozone oxidation at $298 \mathrm{~K}, \mathrm{~J}$. Am. Chem. Soc. 2000, 122, 2383. 


\section{Chapter 5}

\section{Energy Generation and Storage using Functionalized PMMA-CNT Matrices}

\subsection{Introduction to the Energy Sensing, Generation and Storage (SGS) System}

SGS is an abbreviation of sensing, generation and storage system. This was proposed by my advisor, Dr. Yoke Khin Yap, to integrate some of the components of my work using PMMA-CNT matrices including the glucose sensors, glucose biofuel cells, and supercapacitors. SGS is very simple but powerful concept which might be applicable as implantable medical devices in human being. Schematic representation of the SGS concept is shown in Figure 5.1.

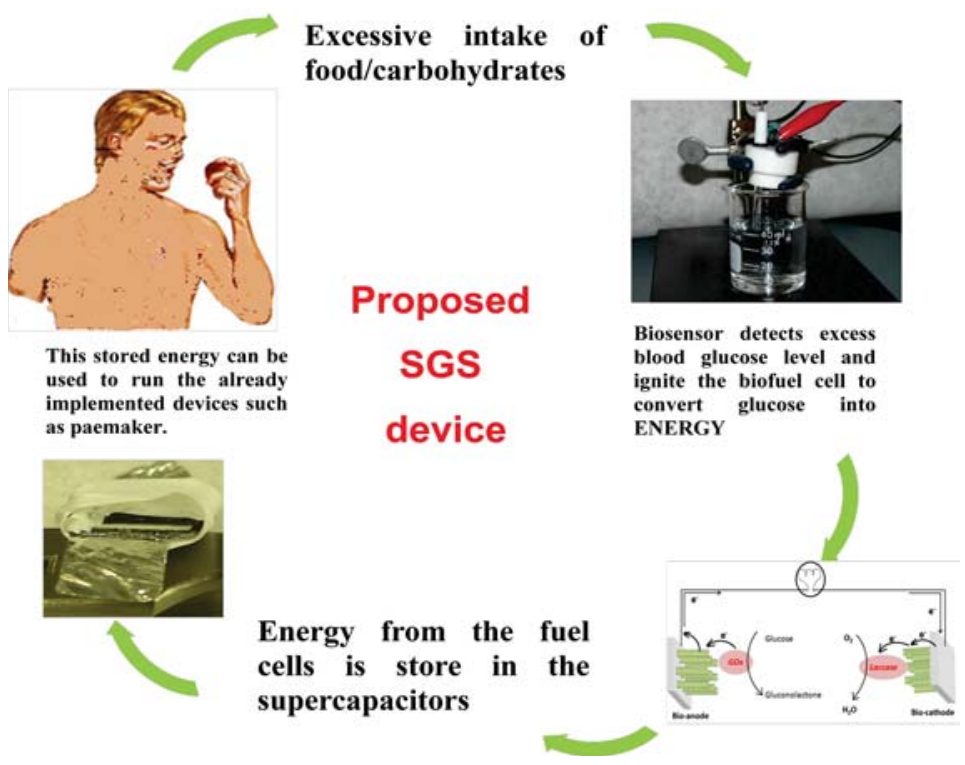

Figure 5.1: Schematic representation of SGS concept 
This system consists of three components: 1) glucose sensors discussed in previous chapter for energy (glucose) "sensing”, 2) glucose BFCs for energy (glucose) "generation", and 3) supercapacitor for energy "storage". The operational concept of SGS is that when the sensors detected a normal blood glucose level, the biofuel cells will not be activated. When the sensors detects a higher than normal blood glucose level, the system will activate the BFCs and convert excessive glucose into energy.

The BFCs will stop generating energy when the glucose level returned to a normal level. The energy generated by the BFCs can be stored in the supercapacitors (or other miniature batteries) to power the glucose sensors and assisting electronics. Alternatively, it can be used to power other implanted devices including pace makers, artificial organs etc.

Thus SGS can help in maintaining normal glucose level in our blood as any excessive glucose will be converted into energy. For those who need extra energy to power other implanted devices, they will need to take the prearranged amount of extra glucose to charge up the supercapacitors. For practical uses, an external monitoring system will be needed to allow users to set alarms to alert them when the blood glucose levels and the energy storage in the supercapacitors are too low or too high.

We have already showed that PMMA-CNT matrices can be used as an excellent glucose biosensor. In this chapter we will show the preliminary results on the other two components of the SGS device energy generation using BFCs and energy 
storage using supercapacitors based on functionalized PMMA-CNT matrices. The details are discussed below.

\subsection{Biofuel cells: A Brief Look}

In view of rising energy demand and due to limited availability of nonrenewable energy resources, there are strong desires to design more efficient sources of alternative renewable energy. This has led to more intense research in alternative, clean and green sources of energy such as BFCs. Renowned Italian scientist Luigi Galvani was the first to observe the twitching of a frog's leg when an electric current was applied [1]. Michael Cresse Potter created the microbial fuel cell by placing a platinum electrode into cultures and created a potential difference [2]. Cohen created high voltage microbial fuel cells [3]. Recently, due to impending energy crisis there has been a spurt in research in BFCs as reported in published excellent reviews on the topic [4]. The next few sections discuss more details about BFCs.

\subsubsection{Introduction: What is biofuel cells (BFCs)}

BFCs are defined as fuel cells where biocatalysts such as microorganisms or enzymes were employed to convert chemical energy into electrical energy by electrochemical routes. In BFCs enzymes are used as a biocatalyst and they can operate under ambient conditions such as $20-30^{\circ} \mathrm{C}$ and neutral $\mathrm{pH}$. The biocatalysts 
can be either protein, enzyme or whole organism and this also offer cost advantage over metallic catalyst such as Pt which is expensive rare earth metal [5].

For decades, microorganisms, which convert foods into electron flows, were only biological interesting. Major interest in BFCs research arose when National Aeronautics and Space Administration in 1960s explored in converting human waste into electricity during their space flights missions. During that period many BFCs which generate power from various organic substances such as urea and methane were demonstrated. Algae and bacteria were among the first organisms used in biological fuel cells. The first enzyme BFC was reported in 1964, which uses an enzyme glucose oxidase $\left(G O_{\mathrm{x}}\right)$ as biocatalyst for anode and glucose as fuel [6]. Many exciting developments have been reported since that time [7-10] but the performance of BFCs in terms of power density, lifetime, and operational conditions, are far below and lags behind that of conventional fuel cells. Now-a-days instead of viewing BFCs as a device for power generation the studies are more directed towards specific applications such as biosensors and implantable devices [11-12].

Advances in nanotechnology have led to a new field of nanomaterials functionalized with biocatalysts such as enzymes. Along with the chemistry of materials many nanostructures evidenced their potential use in activating the enzymes with excellent physical and chemical properties. These nanomaterials have advantage of large surface to volume ratio which is advantageous for immobilization of enzymes and increases the enzyme loading. Enzymes have further advantage of selectivity 
which can eliminate the need for separator membrane. Enzymatic BFCs have disadvantages of having inadequate life time due to the brittle nature of enzyme. A schematic of CNT based enzymatic biofuel cell using glucose and oxygen gas as fuel is shown in Figure 5.2. A typical BFC consists of two electrodes: bioanode and biocathode. Bioanode consist CNTs functionalized with enzyme $G O_{x}$. Biocathode consist CNTs functionalized with enzyme Laccase. Oxidation occurs at the bioanode and the electrons released in the process are then transferred to the biocathode from the external circuit where reduction occurs at the biocathode.

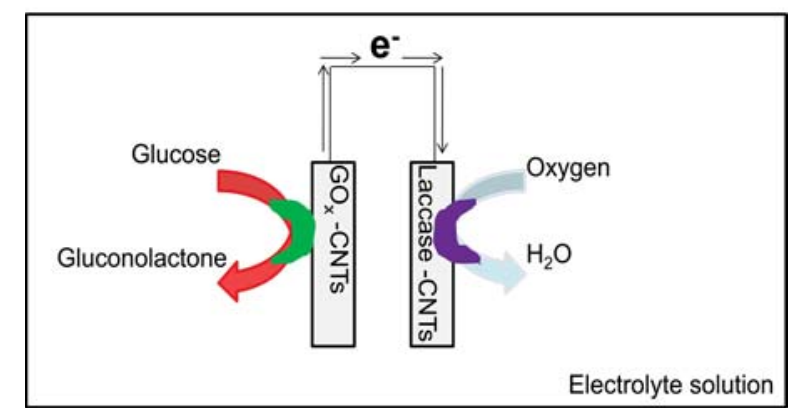

Figure 5.2: Schematic of Enzymatic Biofuel Cells

Among various nanomaterials tested for enzymatic BFCs, CNTs are at the forefront due to their exceptional physical and electrochemical stability. In 2006, it was first reported that $G O_{x}$ immobilized MWCNTs can be used as the bioanode in BFCs [13]. The authors demonstrated direct electron transfer between $G O_{x}$ enzyme and MWCNTs grown on porous carbonaceous matrix with a potential of developing miniaturized membrane-less BFCs. Later, the use of single walled CNTs (SWCNTs) 
based glucose-oxygen BFC with glucose dehydrogenase (GDH) enzyme and laccase enzyme was reported [14]. The authors demonstrated BFCs using paste of GDH functionalized SWCNTs with improved open circuit voltage. Similarly glucose/oxygen BFCs based on paste of $G O_{x}$ functionalized MWCNTs on glassy carbon electrode and paste of laccase functionalized MWCNTs has been demonstrated [15]. Enzymatic BFCs based on SWCNTs modified carbon fiber microelectrodes as a substrate for biocathode and bioanode has also been reported [16]. The authors report high power output and tissue implantable features. Very recently hybrid CNT microwires have been reported for high power BFCs in nature communications [17]. So far most of the efforts in using CNTs have relied on using paste of functionalized CNTs. Based on these nanotubes pastes, promising power density and performance of BFCs have been demonstrated [17]. This chapter presents the use of poly-methyl methacrylate-carbon nanotube (PMMA-CNT) matrices in BFCs and demonstrated the enhanced performance as well.

\subsubsection{Enzyme based BFCs: Related Issues}

Microorganism or enzymes are used as the biocatalysts in enzymatic BFCs. An important development in BFCs was bioanode and biocathode that utilize direct electron transfer (DET) instead of mediated electron transfer from active site of enzyme to electrode [18]. DET between the enzyme and electrode have been observed in many enzymes such as $G O_{x}$, laccase, peroxidase, and hydrogenases [19-21]. The close communication between enzyme active site and the surface of electrode is very 
important in order to achieve DET. The cofactor is located $\sim 13 \AA$ deep inside the enzyme [22-23] hence it is desirable to have the nanoscale electrode so that it can penetrate deep inside the enzyme active site for fast transfer of electrons. Larger distances will degrade the DET efficiency. Enzymes have a complex folded structure which is made of proteins. The electron-transfer unit of the enzyme, called the apoenzyme and the cofactor, are deeply covered inside its complex structure. Due to this in several cases DET is limited and hence the electrical communication between the electrode substrate and the enzyme biocatalyst can be difficult.

Low power density and short enzyme life times are two major issues in realization of practical BFCs. Several research groups have addressed these issues. For example, $G O_{x}$ and peroxidase-11 were used on gold electrodes and gold-cumin BFCs was fabricated. Power density of $520 \mu \mathrm{W} / \mathrm{cm}^{2}$ was observed [24]. Significant improvements were achieved by choosing precise electrode materials and effective means of enzyme immobilization to enhance the transport of electrons between enzyme and the electrode. Enzyme immobilization has been demonstrated using two pathways: physical adsorption and chemical adsorption. Physical adsorption is the most common pathway employed by researchers to immobilize the enzymes on conductive electrode surfaces such as graphite or carbon black. Tarasevich et al. [24] adsorb hydrogenase and laccase on carbon black particles to fabricate glucose $/ \mathrm{H}_{2} \mathrm{O}_{2}$ BFC using ferrocene composite electrode. Minteer et al. [25] used a different approach to immobilize the enzyme on the electrode. The enzyme was entrapped in nafion 
membrane and the enzyme activity was retained using this approach as compared to surface adsorption. These studies emphasized that electron transfer from the enzyme active center to the electrodes is an important factor in improving the power density of BFCs.

One of the other factors which affect the lifetime of BFCs is enzyme stability. Most of the enzymatic fuel cells reported so far can only last for few days [24, 27-29]. Another major issue in demonstration of practical BFC device is power density which is measured by generated power per surface area of the electrode. Higher enzyme loading is very crucial for high power output. This chapter addresses these issues and emphasize on enhancing the parameters for better BFCs.

Excellent chemical and physical properties of CNTs allow them to be used as supports for enzymes to transfer electrons from enzyme to electrode surface. Using VA-MWCNTs can have several advantages over using random CNTs or paste of CNTs. The exposed tips of VA-MWCNTs in our PMMA-CNT matrices allow each CNTs to be functionalized and immobilized with enzymes and enhancing the performance of BFCs. The opened CNT edges may penetrate deep inside the enzyme molecule and reach to the redox active center (co-factor) and provide more efficient transfer of electrons. PMMA-CNT matrices are cleaner and avoid contamination that may degrade the lifetime of the enzymes. PMMA also provides an effective sealing against leakage current from the substrates etc. This also prevent CNTs from disperse 
into the electrolyte solution thereby enhancing the life of the electrode as already demonstrated by use of PMMA-CNT matrices as glucose biosensor in chapter 4 .

\subsubsection{Enzyme used-Laccase}

Our biocathode consist of PMMA-CNT matrices functionalized with enzyme Laccase. Laccase belongs to the enzyme family consisting of multi-copper oxidases.

Figure 5.3 shows the overall structure of laccase enzyme obtained from mushroom Trametes versicolor.

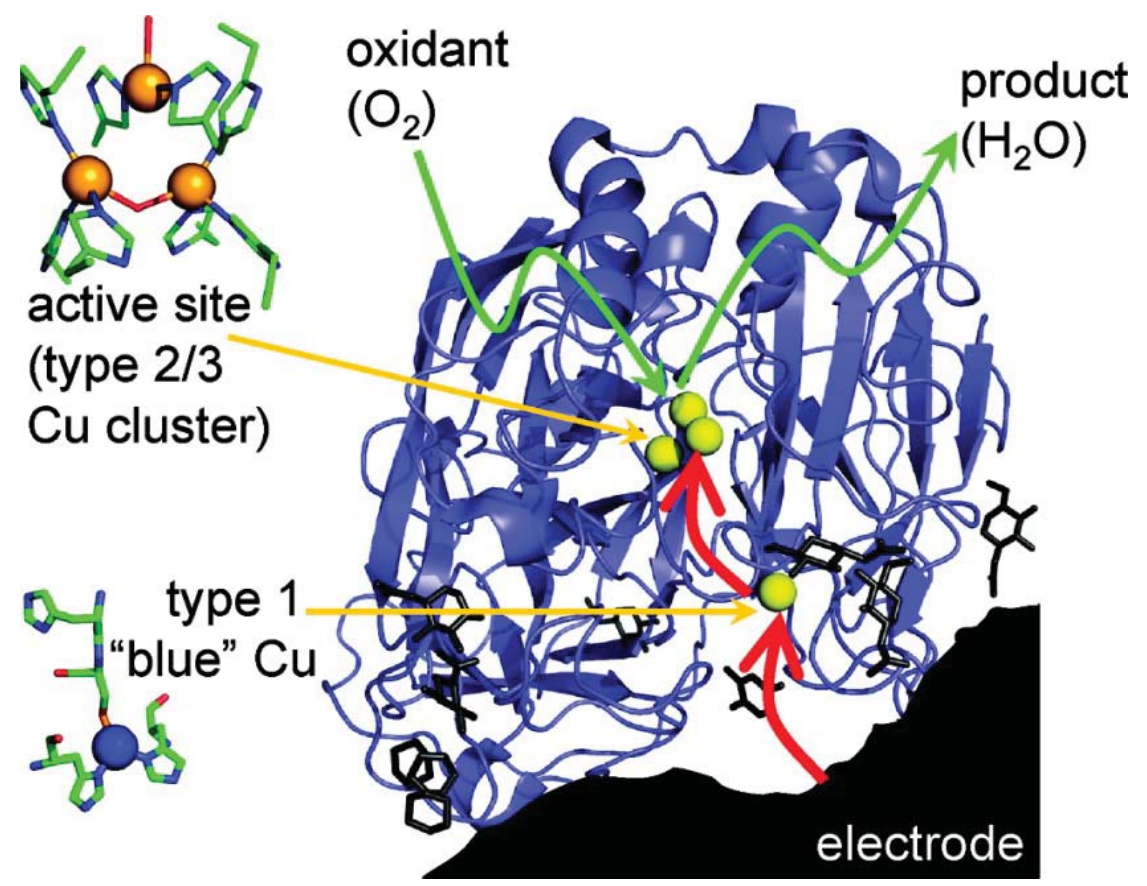

Figure 5.3: Structure and active sites of Laccase from T. versicolor. Reprinted with permission from [30] 
Laccase contains many types of copper sites. It has one type-1 (T1) copper site, which functions as the primary oxidation site. Again it has one type-2 (T2) copper site and two type-3 (T3) copper sites. These sites are arranged in a trinuclear fashion as shown in Figure 5.3. Molecular mass of laccase is $\sim 70 \mathrm{kDa}$. Laccase enzyme can reduce oxygen to water [30]. In the process T1 copper site in laccase gains the electrons first and they are transferred to subsequent to $\mathrm{T} 2$ and $\mathrm{T} 3$ copper sites as indicated by as the arrows in Figure 5.3.

\subsection{Key performance characteristics and terms for BFCs}

In this chapter many different terms have been used which are associated with the BFCs. The description of these terms is as follows:

$V_{\text {Cell }}:$ Potential of the cell or Cell Voltage

$P_{\text {Cell: }}$ : Power output of the cell.

$I_{s c}$ : Short circuit current. A short circuit current is one that permits the flow of current to travel along a path where there is no or very less electrical impedance is present.

$V_{O C}:$ Open circuit voltage $(\mathrm{OCV})$. OCV is defined as difference of electrical potential between two ends of a device when the external circuit is open and no current is flowing.

For any kind of fuel cell the power output, $P_{\text {Cell }}$ is an important parameter in evaluating the performance [32]. 
If $I$ is the current then Power output is given by $P_{\text {Cell }}=V_{\text {Cell }} I$

Again the power density can be calculated as: Power Density $(\mathrm{P})=\frac{P_{\text {cell }}}{A}$

where " $A$ " is the area of the electrode.

Current Density $=$ Current per unit area $=\frac{I_{\text {Cell }}}{A}$

Now electrical work done "W" by an electric charge of "q" coulombs moving through a potential difference " $\mathrm{V}$ " is: $W=q V$

Again charge " $\mathrm{q}$ " can be expressed in terms of " $\mathrm{n}$ " number of moles of electrons transferred by $q=n F$

where F is Faraday's constant (the magnitude of electric charge per mole of electrons. $\mathrm{F}=\mathrm{eN}_{\mathrm{A}}=96485.3399 \mathrm{C} \mathrm{mol}^{-1}$, where charge on single electron is $\mathrm{e}=1.602 \times 10^{-19} \mathrm{C}$ and $\mathrm{N}_{\mathrm{A}}$ is the Avogadro's number.

Therefore using equation (5), equation (4) now becomes $W=n F V$

From thermodynamics we know that maximum electrical energy BFCs can deliver depends on Gibbs free energy, $\Delta \mathrm{G}$ given by: $W=-\Delta G$

Hence thermodynamically predicted voltage output of BFCs, $V=-\frac{\Delta G}{n F}$

The typical polarization curve for any BFCs can be described by Figure 5.4. The fuel cell voltage is on the y-axis and the current density is plotted on the x-axis. 
The voltage output of the real $\mathrm{BFC}$ is less than thermodynamically predicted voltage output (see description hereafter) due to irreversible and various other losses. As shown, there are three major types of biofuel cell losses, which give a biofuel cell $\mathrm{i}-\mathrm{V}$ curve its characteristics shape. Losses in region (1) are due to activation losses. These losses are due to electrochemical reaction.

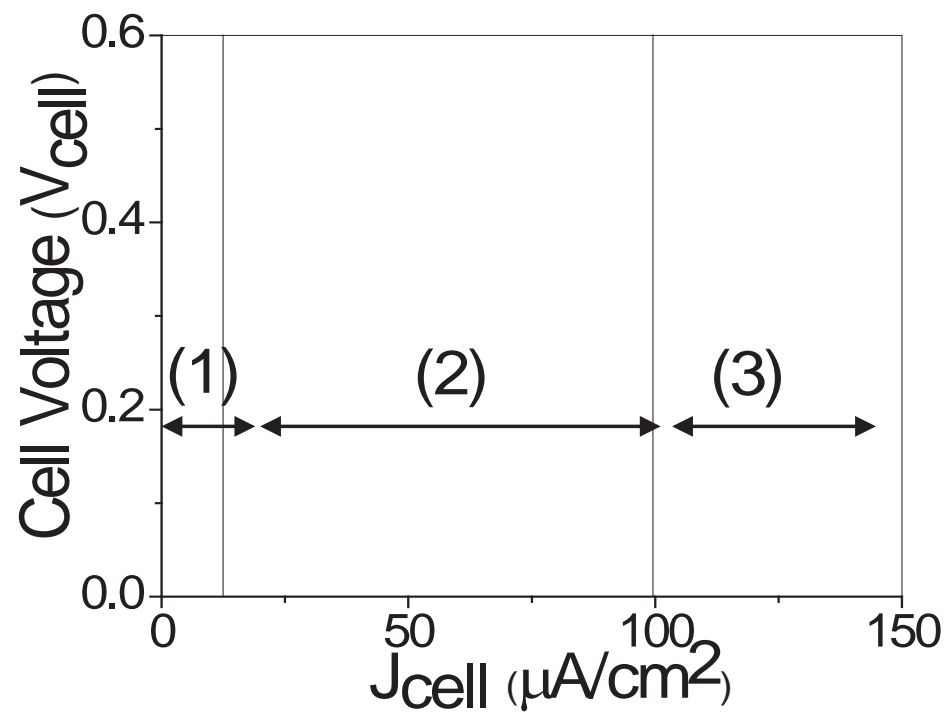

Figure 5.4: Polarization curve

Losses in region (2) are due to ohmic losses. Ohmic losses occur due to the ionic and electronic conduction. Region (3) is concentration losses. These are due to the mass transport. More discussion on losses is given in coming sections [32]. It is observed that as more current is drawn from the cell, the greater these losses and cell potential decreases. 


\subsection{Experimental details-Assembling Membrane-less BFCs}

All the VA-MWCNTs used in this work were grown in circular area of $7 \mathrm{~mm}$ in diameter on low resistance substrates (resistivity $=1 \Omega \mathrm{cm}^{-1}$ ) using PECVD technique. The VA-MWCNTs have diameter of 50-60nm and length is $\sim 4 \mu \mathrm{m}$. After growth of VA-MWCNTs, they were dip coated by Poly methyl methacrylate (PMMA) followed by annealing at $\sim 100^{\circ} \mathrm{C}$. Samples were then polished to expose the tips of CNTs, forming the needed PMMA-CNT matrices as the electrodes of our BFCs. More details on fabrication of PMMA-CNT matrices are given in chapter 3 and chapter 4.

\subsubsection{Fabrication of PMMA-CNT bioanode for BFCs}

The enzyme used for the bioanode was glucose oxidase $\left(G O_{x}\right)$. The detailed structure of the $G O_{x}$ and immobilization process is already described in chapter 4. Before immobilization of $G O_{x}$ on CNTs, the PMMA-CNT matrices were pretreated electrochemically with $1 \mathrm{M} \mathrm{NaOH}$ at $2.8 \mathrm{~V}$ for 5 minutes. This step was done in order to attach carboxylic group $(-\mathrm{COOH})$ on tips of CNTs. Existence of the carboxylic acid group $\left(\sim 1730 \mathrm{~cm}^{-1}\right)$ was confirmed by FTIR spectroscopy. The functionalized matrices were dried for 30minutes in air. Deionized water was used for all the experiments in this work. For the immobilization of $G O_{x}$, we use standard water soluble coupling agents EDC (1-ethyl-3-3-dimethylaminopropyl carbodiimide) and Sulfo-NHS (Nhydroxy-sulfo-succinimide). At first pretreated PMMA-CNT matrices were immersed in $10 \mathrm{ml}$ of $10 \mathrm{mg} / \mathrm{ml}$ aqueous solution of EDC. Next, 300mg of sulfo-NHS was added 
to the above solution with stirring. The reaction was allowed to occur at room temperature for 3 hours. Finally the above samples were washed in DI water and immersed in degassed $G O_{x}$ solution $(2 \mathrm{mg} / \mathrm{ml})$ prepared in $20 \mathrm{ml}$ of $0.1 \mathrm{M}$ phosphate buffer solution ( $\mathrm{pH}$ 7.2). $G O_{x}$ immobilization was allowed to occur at room temperature for 3 hours. The bioanodes were stored at $4^{0} \mathrm{C}$ when not in use.

\subsubsection{Fabrication of PMMA-CNT biocathode for BFCs}

The biocathode of the BFCs was prepared by first dissolving $2 \mathrm{mg}$ of the purified laccase enzyme in $1 \mathrm{ml}$ of PBS. PMMA-CNT matrices were then dipped into this laccase enzyme solution for 2 hours and dried under ambient temperature for about $30 \mathrm{~min}$ and stored at $4^{0} \mathrm{C}$ in a refrigerator.

\subsubsection{Biofuel cell fabrication and characterization}

For assembling a glucose $/ \mathrm{O}_{2} \mathrm{BFC}$, the PMMA-CNTs based bioanode and biocathode were placed in a beaker cell containing 0.1M PBS under ambient air as shown in Figure 5.5.

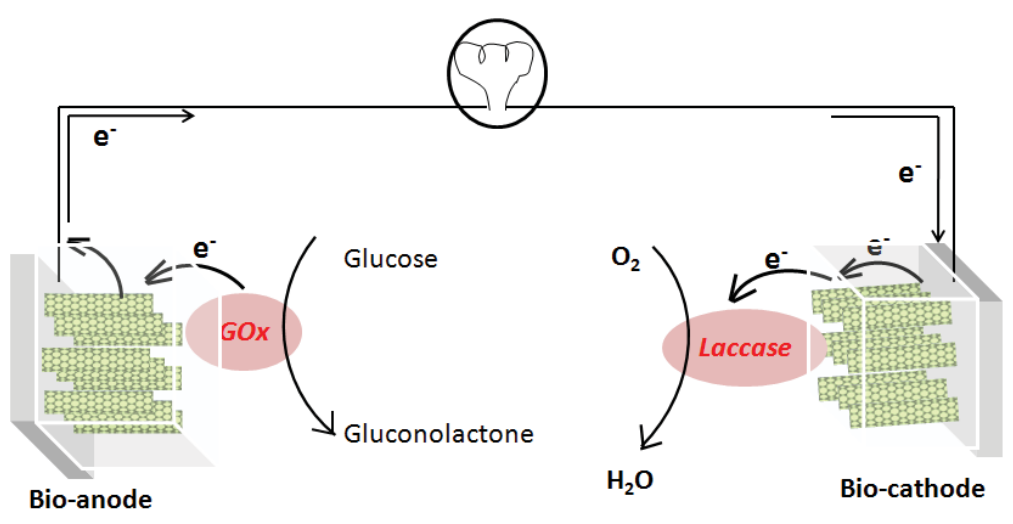

Figure 5.5: Schematic diagram of BFC 
At the anode the electrons are being transferred from glucose to $G O_{x}$ and from $G O_{x}$ to the external circuit (or electrode). At the bio-cathode the electrons are transferred from CNTs to laccase and from laccase to oxygen.

Cyclic Voltammetry (CV) measurements were carried out with a computercontrolled potentiostat (CHI 660A, CHI, Austin TX) in three electrode set-up for both bioanode and biocathode separately. In the three electrodes setup working electrode was the bioanode (or the biocathode), the reference electrode was $\mathrm{Ag} / \mathrm{AgCl}$, and platinum wire was used as counter electrode. $0.1 \mathrm{M}$ Phosphate buffer solution $(\mathrm{pH}=$ 7.2) was used as supporting electrolyte. All the electrochemical measurements were performed at ambient temperature. Current-Voltage polarization measurements on the assembled BFCs were done using CHI 660C potentiostat with the addition of $40 \mathrm{ml}$ of $0.22 \mathrm{mM}$ glucose for all the experiments.

\subsubsection{Fabrication of BFCs with different area}

BFCs with varying electrode area were also fabricated to study the effect of area on performance of BFCs. The details on fabrication of PMMA-CNT matrices with varying electrode area used for bioanode and bio cathode can be found in chapter

4, section 4.3.3. The different electrode areas used in this work are as follows: $0.385 \mathrm{~cm}^{2}, 0.481 \mathrm{~cm}^{2}, 0.587 \mathrm{~cm}^{2}$ and $0.77 \mathrm{~cm}^{2}$. 


\subsection{Results and Discussion: BFCs}

\subsubsection{CV of laccase functionalized PMMA-CNT matrices used as biocathode}

Figure 5.6 shows the $\mathrm{CV}$ for oxygen reduction at the laccase immobilized PMMA-CNT matrices in PBS ( $\mathrm{pH}$ 7.2). As shown, oxygen reduction of laccase occurs at a potential of $0.59 \mathrm{~V}$ versus the $\mathrm{Ag} / \mathrm{AgCl}$ reference electrode. On the hand, the $\mathrm{CV}$ of $G O_{x}$ immobilized PMMA-CNT matrices for use as bioanode in BFCs are already described in detail in chapter 4.

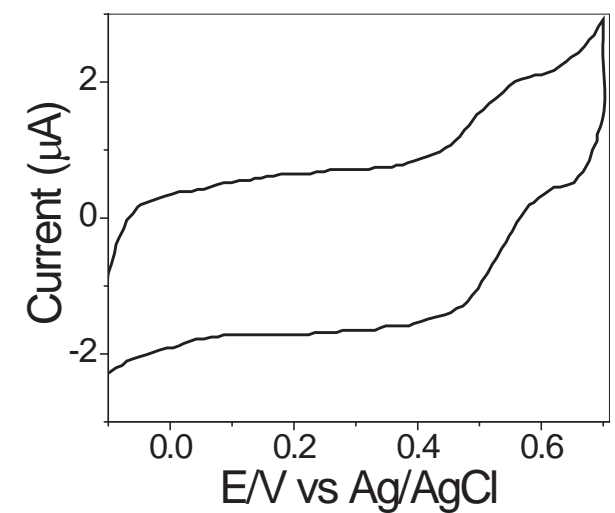

Figure 5.6: $C V$ of Laccase immobilized PMMA-CNT matrices in 0.1M PBS at a scan rate of 5 $\mathrm{mVs}^{-1}$ (Area of working electrode $=0.385 \mathrm{~cm}^{2}$ )

\subsubsection{Polarization measurements of assembled BFCs}

In our biofuel cell, Glucose and oxygen was used as fuel. The redox reaction at the bioanode and biocathode can be described according to the following equation:

Bioanode (oxidation of glucose) $: G O_{x}+2$ Glucose $\longrightarrow 2$ gluconolactone $+4 \mathrm{H}^{+}+4 \mathrm{e}^{-}$

Biocathode (reduction of oxygen): $\mathrm{O}_{2}+4 \mathrm{H}^{+}+4 \mathrm{e}^{-} \longrightarrow 2 \mathrm{H}_{2} \mathrm{O}$ 
Overall redox reaction is:

$$
\text { 2Glucose }+\mathrm{O}_{2} \longrightarrow 2 \text { Gluconolactone }+2 \mathrm{H}_{2} \mathrm{O}
$$

In glucose $/ \mathrm{O}_{2} \mathrm{BFC}$, glucose is oxidized at the bioanode according to equation (1). At the biocathode the oxygen is reduced to the water according to equation (2). The overall redox equation is given in equation (3).

The typical polarization curve, and the dependence of the power output on the current density of our glucose $/ \mathrm{O}_{2}$ BFCs are shown in Figure 5.7 (a) and (b), respectively. As shown in Figure 5.7 (a), the open circuit voltage (at $\mathrm{J}_{\text {cell }}=0$ ) obtained was $0.54 \mathrm{~V}$. A short circuit current density $\left(\mathrm{I}_{\mathrm{SC}}\right)$ of $154 \mu \mathrm{A} / \mathrm{cm}^{2}$ was obtained (at $\mathrm{V}_{\text {cell }}=0$ ). The characteristic shape of the polarization curve is due to various irreversible losses.

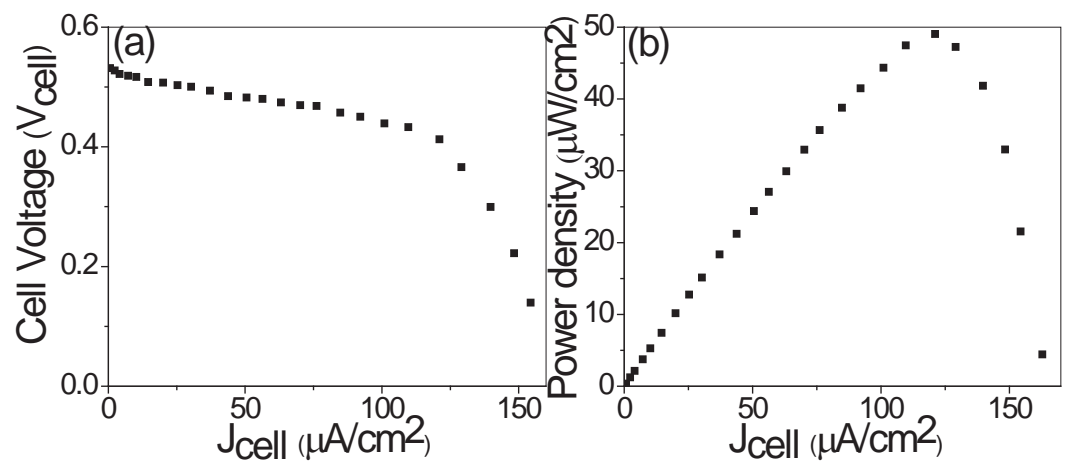

Figure 5.7: (a) Polarization curve and (b) Power output of glucose/O $\mathrm{O}_{2}$ BFCs with $\mathrm{GO}_{x^{-}}$ PMMA-CNT matrices as anode and Laccase-PMMA-CNT matrices as cathode respectively.

As shown in Figure 5.7 (b), the maximum power density was $48.9 \mu \mathrm{W} / \mathrm{cm} 2$. The initially zero power density increases as the current density increases up to a 
maximum density and decreases beyond that due to ohmic and various other losses. More detailed discussion on various losses is given in next section.

\subsubsection{Effect of different electrode area on performance of BFCs}

In order to see the effect of device area on performance of BFCs, we have fabricated bioanodes and biocathodes with different areas. The different areas used in this work are as follows: $0.385 \mathrm{~cm}^{2}, 0.481 \mathrm{~cm}^{2}, 0.587 \mathrm{~cm}^{2}$ and $0.77 \mathrm{~cm}^{2}$. Figure 5.8 shows the $\mathrm{CV}$ for oxygen reduction at the laccase immobilized PMMA-CNT matrices for areas (a) $0.481 \mathrm{~cm}^{2}$, (b) $0.587 \mathrm{~cm}^{2}$ and (c) $0.77 \mathrm{~cm}^{2}$ respectively in PBS (pH 7.2). Shape of CV remains the same with slight increase in anodic current level. This is understandable since due to increase in area more number of Laccase functionalized sites is available for reduction of oxygen.
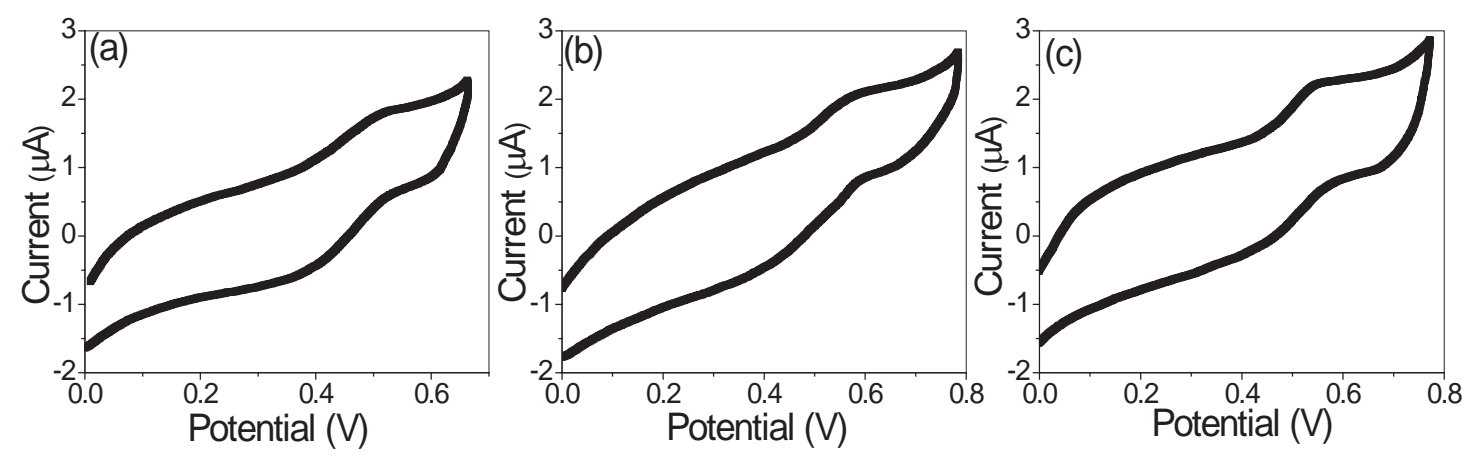

Figure 5.8: CV of Laccase immobilized PMMA-CNT matrices used as biocathode for areas (a) $0.481 \mathrm{~cm}^{2}$, (b) $0.587 \mathrm{~cm}^{2}$ and (c) $0.77 \mathrm{~cm}^{2}$ 

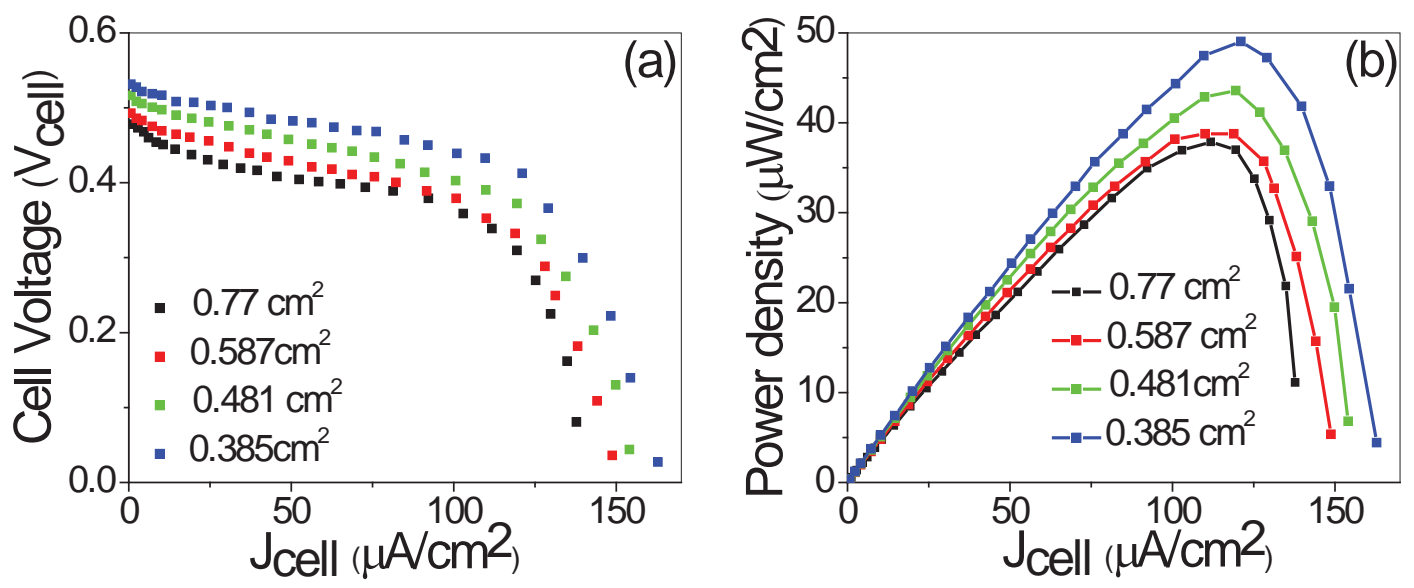

Figure 5.9: (a) Polarization curves and (b) Power output of PMMA-CNT matrices based glucose $/ \mathrm{O}_{2} \mathrm{BFCs}$ for varying electrode areas $0.385 \mathrm{~cm}^{2}, 0.481 \mathrm{~cm}^{2}, 0.587 \mathrm{~cm}^{2}$ and $0.77 \mathrm{~cm}^{2}$.

Figures 5.9 (a) and (b) shows the variation of polarization and power density for the assembled glucose $/ \mathrm{O}_{2}$ BFCs for increasing electrode area. It is observed that both $\mathrm{V}_{\mathrm{OC}}$ and $\mathrm{J}_{\mathrm{SC}}$ decrease with the increase of device area. This is due to increase in various losses with increase in device area and also due to the fact that number of electroactive sites does not increase in same ratio as area increases.

Table 5.1 shows the variation in performance parameters of BFCs as a function of device area. As shown, the open circuit voltage, the short circuit current density, and the maximum power density decrease with the decrease of device area. This can be attributed to increase in losses and relative decrease in number of electroactive enzyme sites per unit area as electrode area is increased. In a fuel cell three types of losses can occur. 
Table 5.1: Variation in performance parameters of BFCs with different electrode areas

\begin{tabular}{|c|c|c|c|}
\hline $\begin{array}{c}\text { Area } \\
\left(\mathrm{cm}^{2}\right)\end{array}$ & $\begin{array}{c}\text { Open circuit } \\
\text { voltage } \\
(\mathrm{V})\end{array}$ & $\begin{array}{c}\text { Short circuit current } \\
\text { density } \\
\mu \mathrm{A} / \mathrm{cm}^{2}\end{array}$ & $\begin{array}{c}\text { Maximum Power } \\
\text { density } \\
\mu \mathrm{W} / \mathrm{cm}^{2}\end{array}$ \\
\hline 0.385 & 0.54 & 154 & 48.9 \\
\hline 0.481 & 0.53 & 151 & 43.5 \\
\hline 0.587 & 0.49 & 148 & 42.4 \\
\hline 0.77 & 0.47 & 139 & 38.8 \\
\hline
\end{tabular}

First type is loss due to reaction kinetics which is known as activation loss. Second type of loss is known as Ohmic loss due to resistances to conduction. Third type of loss is due to mass transport known as concentration loss. We will consider the effect of each type losses on cell Voltage when electrode area is increased one by one.

(1) Activation loss: Electrochemical reactions involve the transfer of charges between an electrodes and chemical species. Thus current evolved in electrochemical reactions is a direct measure of the rate of electrochemical reaction. Since the rate of an electrochemical reaction is finite and hence the 
current produced is also limited. The reactants can convert into current only after crossing an energy barrier also known as activation energy barrier. In fact, an electrochemical reaction occurs in a series of steps for example electrochemical reaction occurring on laccase biocathode involves electron transfer from the electrode to laccase enzyme site $\mathrm{T} 1$ and then to T2/T3 etc. The net rate of a reaction is given by the difference between forward and reverse reaction rates on the bioanode and biocathode. Activation loss in BFCs refers to the voltage loss which occurs to overcome the activation barriers of the electrochemical reactions on both the electrodes. Now the magnitude of activation loss depends on current density and exchange current density [32].

$\eta_{a c t}=\frac{R T}{\alpha n F} \ln \left(\frac{J}{J_{0}}\right)$

Where $\alpha$ is transfer coefficient, $\eta_{a c t}$ activation loss, $J$ is current density and $J_{0}$ is exchange current density.

Exchange current density is the current density at thermodynamic equilibrium when forward and reverse reaction rates are same and equal to exchange current density. One of the suggested ways to increase exchange current density is to increase the number of reaction sites per unit area [32]. In our case, although the device areas were increased but the number of reaction sites per unit area would nearly remain the same as they are functionalized in the same way. Hence as the area increases activation loss remains nearly the 
same. This interpretation is consistent to the identical gradients on all the curves as shown within the range of $0-15 \mu \mathrm{A} / \mathrm{cm}^{2}$ in Figure 5.9.

(2) Ohmic Loss: Compared to activation loss ohmic loss is more straightforward. Ohm's law which relates potential (V) to current (i) and resistance (R) is defined as

$$
V=i R
$$

Now electrochemical reactions take place at the electrode-electrolyte interfaces so current obtained is directly proportional to the area. Hence, when comparing fuel cells current density is more appropriate for specification than current. Therefore we can modify ohm's law to represent current density $(j)$ as follows:

$$
V=\frac{i}{A} A R=j(A R)
$$

Quantity " $A R$ " is known as area specific resistance i.e. resistance multiplied by area. Now area specific resistance increases with the increase of device area. However resistance is inversely proportional to area according to the equation

$$
R=\rho\left(\frac{L}{A}\right)
$$

Therefore the area specific resistance for fuel cells with different electrode areas is the same. This interpretation is consistent to the identical gradients on all the curves as shown within the range of $\sim 25-100 \mu \mathrm{A} / \mathrm{cm}^{2}$ in Figure 5.9. 
(3) Concentration Loss: Concentration loss occurs mainly at high current densities and is related to rate of mass transport and diffusion. Electrochemical reaction occurs at the interface of catalyst layer on electrode and electrolyte. This leads to reactant depletion and product accumulation at the catalyst surface and sets up a concentration gradient. Concentration leads to diffusive mass transport of reactant to the catalyst layer from surrounding electrolyte solution and vice-versa for the products. Eventually a steady state is reached. This can be represented in terms of current density obtained by the following equation:

$$
j=n F J_{\text {diff }}
$$

Where $j$ is current density obtained from the fuel cell, " $\mathrm{J}_{\text {diff }}$ "is diffusion flux of reactants towards catalyst, $\mathrm{n}$ is number of electrons transferred in the electrochemical reaction and $\mathrm{F}$ is Faraday constant

Now Fick's first law of diffusion can be applied for diffusive mass transport flux. Therefore,

$$
J_{\text {diff }}=-D\left(\frac{d c}{d x}\right)
$$

Where $D$ is reactant diffusivity and $d c / d x$ is concentration gradient of reactant. Concentration gradient can be expressed as reactant concentration (glucose concentration) at the catalyst layer $\left(C_{R}\right)$ and in bulk $\left(C_{R}{ }^{0}\right)$, diffusion layer thickness (d) 
(thickness across which the concentration gradient exists) and $D$ is reactant diffusivity. Therefore equation (4) can be rewritten as:

$$
J_{\text {diff }}=-D\left(C_{R}-C_{R}^{0}\right) \frac{1}{d}
$$

Combining equation (10) and (13) and reorganizing we get:

$$
C_{R}=C_{R}^{0}-(j d) / n F D
$$

Hence as current density, $j$ increase with the increase of reactant depletion, i.e. $C_{R}$ decrease more rapidly and more is the concentration loss. Hence concentration loss is dependent on the diffusive mass transport properties rather than the area of the working electrode. This interpretation is consistent to the identical gradients on all the curves as shown within the range of $\sim 115-150 \mu \mathrm{A} / \mathrm{cm}^{2}$ in Figure 5.9.

Apart from the abovementioned losses we also observed that $\mathrm{V}_{O C}$ decrease slightly with increase in electrode area which might be due internal current leakage from the electrodes and other losses as well.

Apparently, all the possible losses described earlier cannot explain results shown in Figure 5.9. In fact, one can observe that all the curves in Figure 5.9 (a) are merely differentiated by the decrease of $\mathrm{V}_{\mathrm{OC}}$ with the increase of electrode area. Thus we attempt to explain this as follows. As discussed, the redox processes occurring in the cell is described in equations (1) and (2), which involve oxidation/reduction of glucose $/ \mathrm{O}_{2}$ fuel molecules at an enzyme active site. Obviously, these processes depend 
on the access of glucose and $\mathrm{O}_{2}$. As illustrated in Figure 5.10, the functionalized CNTs at the centre of the electrodes have the access to glucose $/ \mathrm{O}_{2}$ fuel molecules in solution from one direction. On the other hand, the functionalized CNTs at the edges of the devices have additional access of glucose $/ \mathrm{O}_{2}$ fuel molecules from other directions.
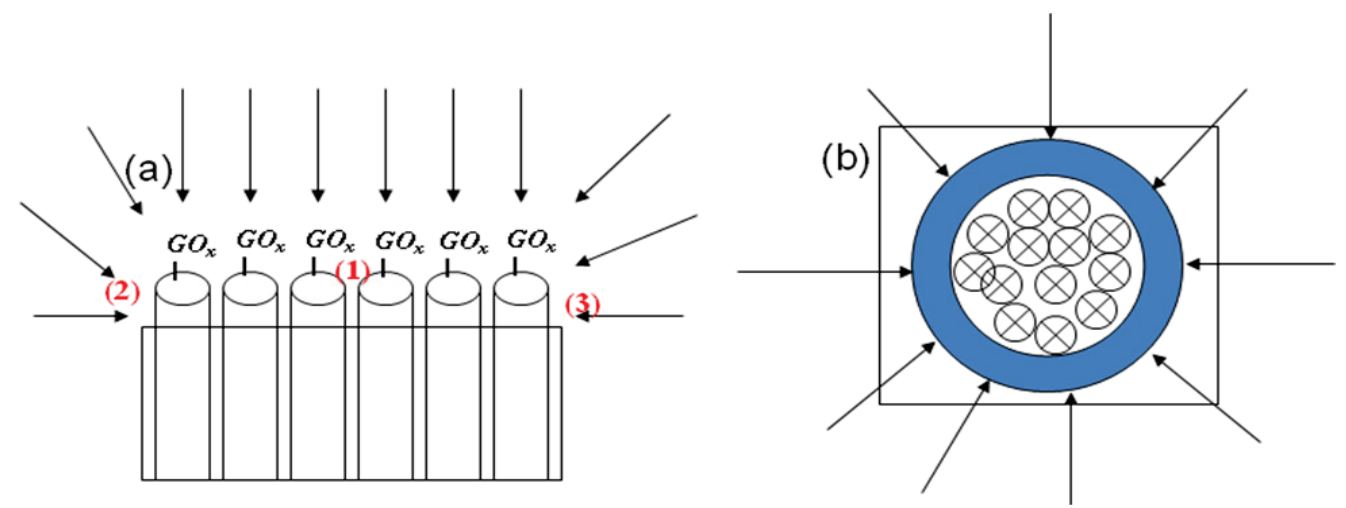

Figure 5.10: Schematic of fuel flow from different directions towards the electrodes (a) $3 D$ view and (b) cross section view

As shown, the flux of fuel to the centre of the electrode at point 1 is unidirectional whereas for CNTs on the side at points $2 \& 3$ fuel flow is from various directions. This means, the electrochemical reaction will be more intense at the edges of the BFCs. Since devices with smaller area have higher portion of CNTs at the edges than CNTs at the centre. This provides less internal impedance for the redox process to occur on smaller devices. This means, the potential drop due to this internal impedance is will be higher for BFCs with larger electrode area. Thus increasing the electrode area of the BFCs will decrease the $\mathrm{V}_{\text {cell }}$ and power density as observed in Figure 5.9. Overall polarization curve is the combination of all the losses. Hence the overall performance of the assembled biofuel cell decreases with increase in electrode area [32]. 


\subsection{Supercapacitors as Energy Storage Devices: An Introduction}

Supercapacitors comprises of two conducting electrodes which are separated by a semi-permeable membrane and immersed in an electrolyte. Semi-permeable membrane separates ions and avoids electrical short circuit between the two conducting electrodes.

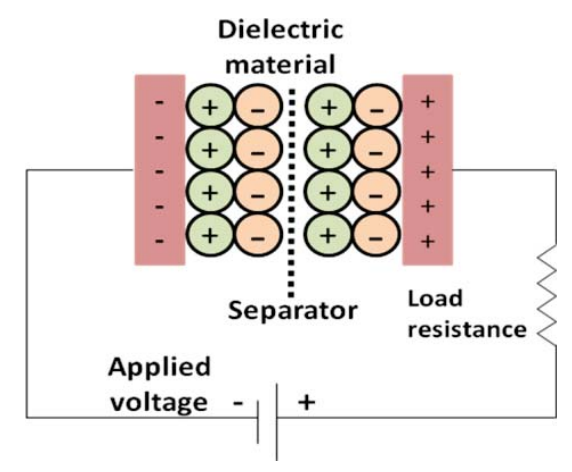

Figure 5.11: Schematic diagram of supercapacitor

Figure 5.11 shows the component of a supercapacitor. It is also known as Electrochemical Double Layer Capacitors (EDLC). Supercapacitors refer to a class of efficient energy storage devices which utilize extremely high active surface area of conducting electrodes separated by a separator and an ultrathin layer of electrolyte. Supercapacitors have extremely high capacitances when compared with the conventional capacitors [33-40]. Three kinds of supercapacitors have been studied most: metal oxide based [41-42], conducting polymer based [43], and carbon based supercapacitors [44]. Carbon based supercapacitors have been studied most widely because of their lower cost, longer life cycles and higher value of capacitance. 
EDLC uses an electrochemical double layer of charges or ions to store electrical energy. When external voltage is applied between electrodes, opposite charges accumulate on the surfaces. Attraction between opposite charges causes the ions present in the electrolyte solution to move across the separator and collect into the electrodes of the opposite polarity. Electrostatic interface has a double layer between charges $(Q)$ on electrode and ions. The capacitance (denoted by " $C$ ") of any material is given by the formula:

$$
C=\frac{Q}{V}
$$

where $V$ is the potential on the electrode. For the conventional parallel plate capacitor, $C$ is directly proportional to the area of the electrode and inversely proportional to the distance between the electrodes. So equation 15 can be rewritten as:

$$
C=\frac{\varepsilon_{0} \cdot \varepsilon_{r} \cdot A_{\text {Electrode }}}{D}
$$

Where $A_{\text {electrode }}$ is the area of the electrode, $\varepsilon_{0}$ is the permittivity of the free space, $\varepsilon_{r}$ is the relative permittivity of the dielectric material and $D$ is the distance between the two electrodes. Energy $(E)$ stored in a capacitor is

Given by the formula: $E=\frac{C V^{2}}{2}$

There are many reasons why CNTs based supercapacitors might outperform activated carbon in conventional capacitors [45]. CNTs have good conductivity, large 145 
surface area (1 to $>2000 \mathrm{~m}^{2} / \mathrm{g}$ ), high temperature stability, and percolated pore structure that can be modified to optimize their properties. Multi-walled CNTs have specific capacitances of 4 to $135 \mathrm{~F} / \mathrm{g}$ as determined by $\mathrm{CV}$ and dc-discharge using $\mathrm{KOH}$ as an electrolyte [46-47]. Supercapacitors with CNTs [48] can be operated at high current level with fast charge-discharge rate [49]. Niu et al. [50] reported the use of CNTs in making electrochemical supercapacitors with high capacitance and high power density. Many researchers have reported the excellent electrochemical supercapacitors with the use of CNTs [52-57]. All these reports show that with the use of CNTs has led to supercapacitors that have a high capacitance and high energy density.

Not only pure MWCNTs or SWCNTs but composites of CNTs coated with metal oxides such as Ruthenium oxide [57], Cobalt Oxide [58], Manganese oxide [59], Nickel hydroxide [60], Vanadium pentoxide [61] and Titanium oxide [62], Tin oxide [62] with excellent capacitance values have also been reported.

Composites of polymer and CNTs were used for supercapacitors in the past few years. In one of the early works, poly(3,4-ethylenedioxythiophene) (PEDOT)/CNT composite for supercapacitors [63-64] was reported with a high capacitance of $60 \mathrm{~F} / \mathrm{g}$ to $160 \mathrm{~F} / \mathrm{g}$ with good cycling performance. Again supercapacitor made from composite of MWCNTs and Polyacrylonitrile (PAN) polymer blend have also been reported with remarkable capacitance properties with high energy density [65]. On a similar note Polyaniline (PANI) has been frequently reported for use as 
composite with CNTs for supercapacitors. PANI/MWCNTs composites have been fabricated with different techniques such as oxidative polymerization [66-67] and microwave assisted synthesis [68]. High capacitance values of 328 F/g [66], 322 F/g [68] and 224 F/g [67] with excellent energy density have been reported. Again PANI/SWCNT composites have also been reported with a high values of $485 \mathrm{~F} / \mathrm{g}$ [69] and 311.7F/g [70] with excellent energy density and power density values. Another conducting polymer reported for use as composite with MWCNTs for supercapacitor is Polypyrrole (PPy). High specific capacitance of $192 \mathrm{~F} / \mathrm{g}$ is reported with PPy/MWCNT composites [71]. PPy/SWCNT composite has been reported with a high capacitance of 265 F/g [72]. Supercapacitors with PPy/MWCNTs as negative and PANI/MWCNTs as positive electrodes with high capacitance of $320 \mathrm{~F} / \mathrm{g}$ have also been reported [73]. But PMMA has never been tested for use as composite with CNT for supercapacitor since they are not conducting. In the following section I will present my results on use of PMMA-CNT matrices as an electrode for supercapacitor. Our approach is different from other CNT composites as we are using opened-tip VAMWCNTs that have good electric contact with the substrates.

\subsection{Experimental: Fabrication of PMMA-CNT matrices based supercapacitor}

Detailed growth of CNTs using PECVD is discussed in previous chapters. PMMA-CNTs based supercapacitors basically have three components: Two electrodes, separator and current collector. 

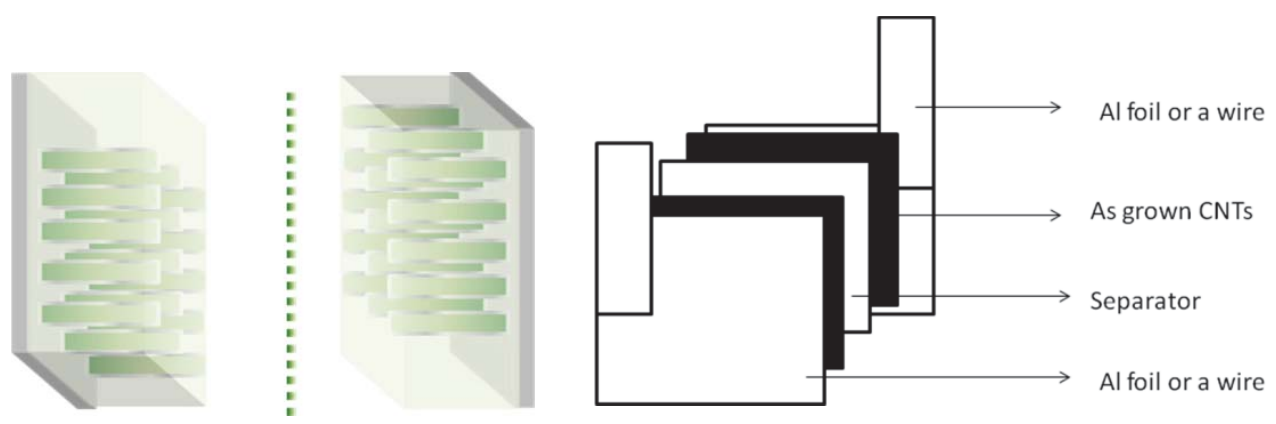

Figure 5.12: (a) Schematic diagram of PMMA-CNTs based supercapacitor and (b) different components of the supercapacitors

Figure 5.12(a) shows the schematic diagram of the PMMA-CNT matrices based supercapacitor. Figure 5.12(b) shows all the components used in the supercapacitor. Two samples of PMMA-CNT matrices were used as active electrodes. Aluminum sheet $(2 \mathrm{~cm} \times 1 \mathrm{~cm})$ was used as the current collector and Whatman filter paper (\# 42) was used as separator material for supercapacitor. The electrolyte used in this experiment was phosphate buffer solution ( $\mathrm{pH} 7.2)$.
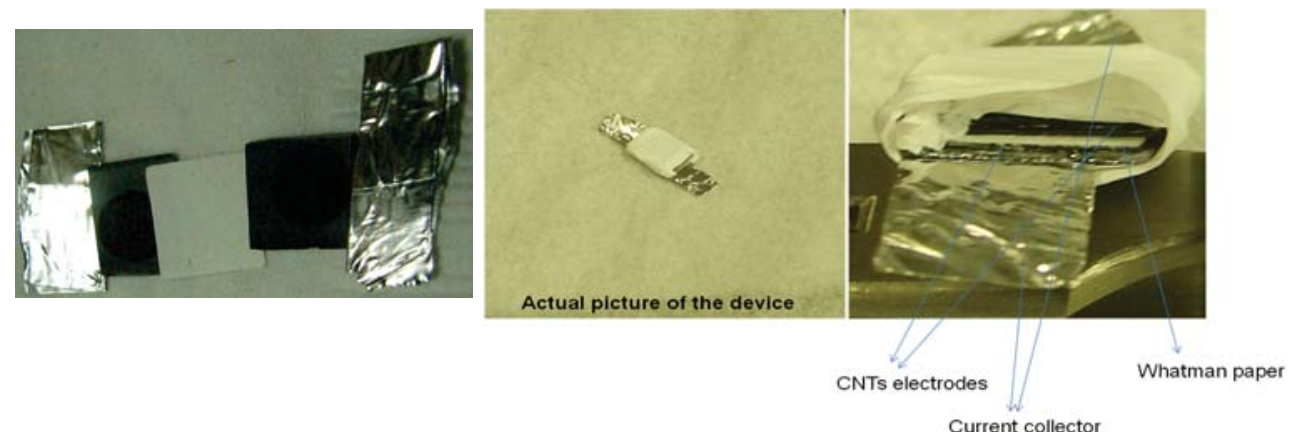

Figure 5.13: (a) Picture showing different component of the supercapacitors (b) Picture of PMMA-CNT supercapacitor and (c) Enlarged picture showing different components of the supercapacitor 
The aluminum foil was stick in the back side of the PMMA-CNT electrode using a drop of silver paste. Next the Whatman filter paper separator soaked in $0.1 \mathrm{M}$ PBS electrolyte ( $\mathrm{pH}$ 7.2) was sandwiched between the two electrodes. The entire assembly was pressed and wrapped around the sides with teflon tape as shown in Figure 5.13(a), (b) and (c).

For electrochemical measurements of the PMMA-CNTs supercapacitor, cyclic voltammetry $(\mathrm{CV})$ and Galvanostatic charge-discharge was analyzed in two electrode setup using the potentiostat/galvanostat, $\mathrm{CHI} 660 \mathrm{C}$ instrument.

\subsection{Results and Discussion: Performance of PMMA-CNT Supercapacitors}

CV of PMMA-CNT based electrochemical supercapacitor was measured. The PMMA-CNT supercapacitor shows near-rectangular CV behavior which is typical of capacitive systems as shown in Figure 5.14.

The potential scan range was $0 \mathrm{~V}$ to $1.0 \mathrm{~V}$ with varying scan rates of 100 $\mathrm{mV} / \mathrm{s}, 250 \mathrm{mV} / \mathrm{s}, 500 \mathrm{mV} / \mathrm{s}$ and $1000 \mathrm{mV} / \mathrm{s}$. CV shapes show a rapid current response when voltage is reversed and is able to maintain capacitive behavior even at high potential scan rates. Capacitance "C" can be estimated from these curves by the following formula [73]:

$$
C=\frac{I_{a v g}}{S}
$$



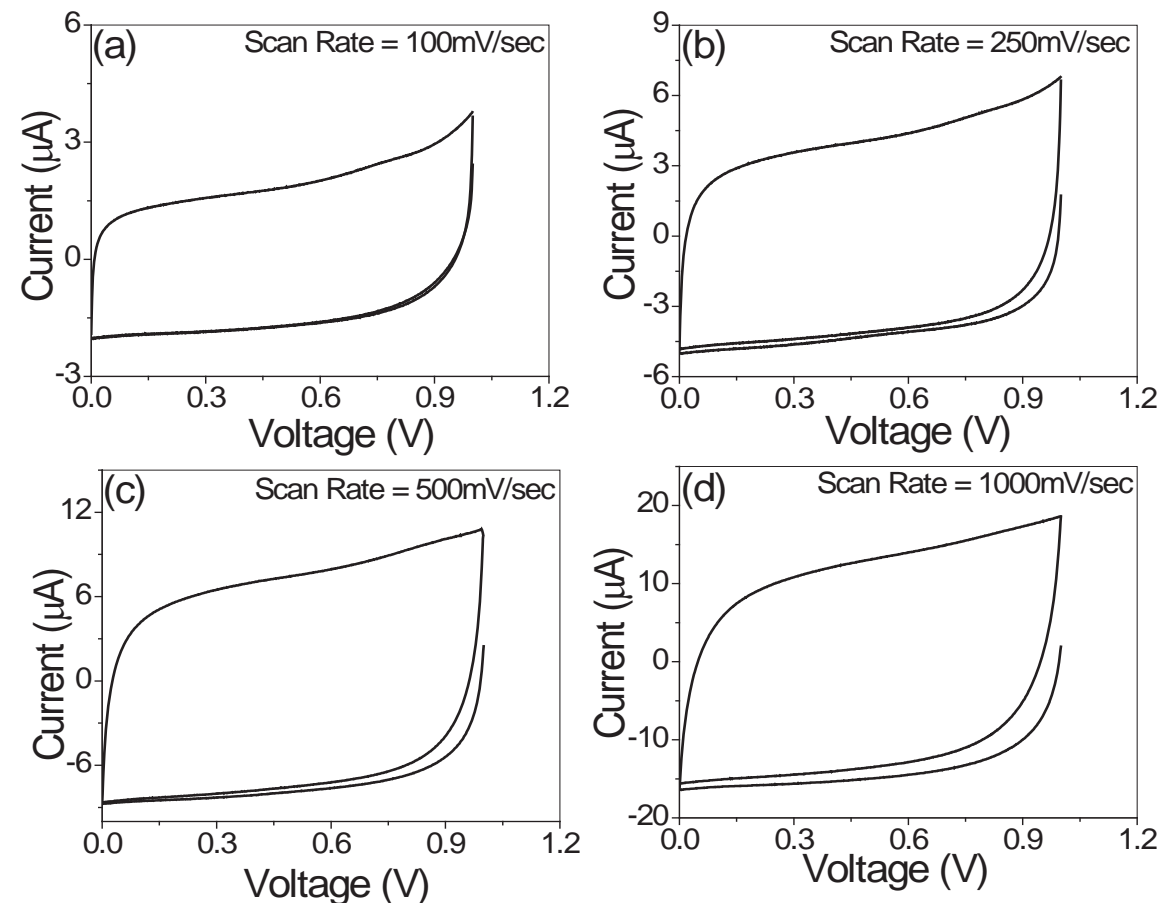

Figure 5.14: CV of the PMMA-CNT matrices based supercapacitors with scan rate of (a) $100 \mathrm{mV} / \mathrm{sec}$, (b) $250 \mathrm{mV} / \mathrm{sec}$, (c) $500 \mathrm{mV} / \mathrm{sec}$ and (d) $1000 \mathrm{mV} / \mathrm{sec}$.

where $I_{\text {avg. }}$ is the average current in either forward scan or reverse scan and "s" is the scan rate. Estimate of $\mathrm{C}$ from curves in Figure 5.14 gives value from 14-20 $\mu \mathrm{F}$.

Current charge/discharge behavior of the PMMA-CNT supercapacitor was observed in the galvanostatic charge-discharge cycling as shown in Figure 5.15. The charge-discharge measurement was carried out at a constant current of $10 \mu \mathrm{A}$ and an operational voltage varying from $0 \mathrm{~V}$ to $1 \mathrm{~V}$. The slope of the discharge curve can be used to calculate the specific capacitance (capacitance per gram) by using the formula below [55]: 


$$
C=\frac{I}{d V / d t}\left(\frac{2}{m}\right)
$$

where, $I$ is the discharge current, $\mathrm{dV} / \mathrm{dt}$ is the slope of the discharge curve, and $\mathrm{m}$ is the weight of the active material of PMMA-CNT electrodes. Approximate weight of CNTs is $\sim 3.6 \mathrm{mg}$. This was calculated by comparing mass of Ni coated $1 \mathrm{X} 1 \mathrm{~cm}^{2} \mathrm{Si}$ substrate before and after the after the growth of CNTs. Again the actual weight of CNTs in PMMA-CNT matrices is even lower after the polishing step since tips of CNTs are polished away. Therefore approximately $\mathrm{m}=3.6 \mathrm{mg}$ - just for the CNTs. The slope of discharge curve is $=0.819 \mathrm{~V} / \mathrm{sec}$. The specific capacitance $6.7 \mathrm{mF} / \mathrm{g}$ was calculated using the above formula. This is close to what was estimated from the CV 14-20 $\mu \mathrm{F}$ per $3.6 \mathrm{mg}=3.9-5.6 \mathrm{mF} / \mathrm{g}$. The specific energy density at $\mathrm{V}=1 \mathrm{~V}$ is from calculated equation (19) to be $3.35 \mathrm{~mJ} / \mathrm{g}$.

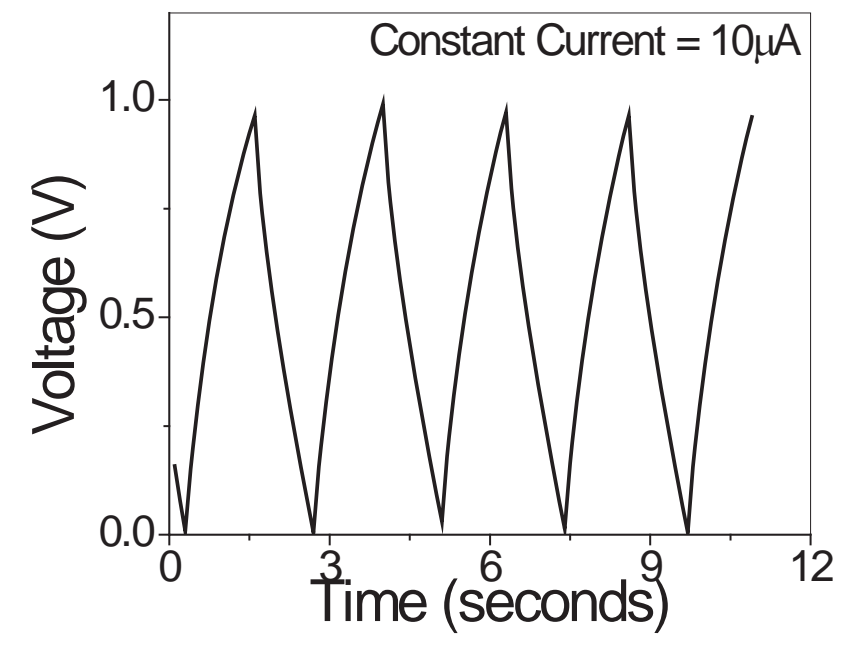

Figure 5.15: Galvanostatic charge-discharge curve of the PMMA-CNT matrices based supercapacitor at a constant current of $10 \mu \mathrm{A}$. 
Power density can be calculated using the following equation

$$
P=\left(\frac{E}{\Delta t}\right)
$$

The value of power density is calculated to be $2.23 \mathrm{~kW} / \mathrm{kg}$ for $\Delta \mathrm{t} \sim 1.5$ seconds. These values are less appealing when compared to other CNT based supercapacitors and other supercapacitor reported in literature [56-73]. There are several reasons for this low performance.

First, most of the reported CNT based supercapacitors are based on paste of CNTs or randomly grown CNTs covering the whole area of the electrode. In our case only very small portion of the electrode was covered with CNTs (only $0.385 \mathrm{~cm}^{2}$ of CNTs on $1 \mathrm{~cm}^{2}$ substrate). Therefore contribution of CNTs towards enhancement of capacitance was much less.

Secondly, the capacitance contribution from the remaining area $\left(1 \mathrm{~cm}^{2}-0.385\right.$ $\left.\mathrm{cm}^{2}=0.615 \mathrm{~cm}^{2}\right)$ is not enhanced as the area is plain and no increase in surface area due to absence of CNTs.

Thirdly the amount of CNTs in each electrode was only $3.6 \mathrm{mg}$ when compared to the total weight of the electrode. More CNTs loading will lead to enhancement in surface area and more EDLC. Furthermore, only the tips of these CNTs are exposed to charges and thus offered significantly small portion of surface area. 
Also small leakage currents and high contact resistances can also cause poor performance. So although this proves that PMMA-CNT matrices can be used for supercapacitors but in its present form PMMA-CNT matrices are not optimized for supercapacitors. There are several ways to enhance the performance which is discussed in future works section. However, the major advantage offered by PMMA-CNT matrices is that CNTs are securely attached to the substrate and become more bio-compatible as the chances to have loosely bonded CNTs are low.

\subsection{Conclusions}

(1) We demonstrated the use of PMMA-CNT matrices functionalized for use in glucose $/ \mathrm{O}_{2}$ BFCs with $G O_{x}$ and laccase enzymes as the biocatalysts. The use of MWCNTs facilitates direct electron transfer of laccase based cathode. We also studied the effect of active electrode area on the performance of BFCs parameters. Overall performance of the assembled biofuel cell decreases with increase in electrode area. This has lead to two important conclusions: 1) miniature BFCs are optimum for future implantable devices for the energy SGS system we proposed here; 2) scaling up the active area of the BFCs for large scale energy generation is not the most efficient way to improve performance unless we increase the electro active enzyme per unit area and reduce the losses. 
(2) PMMA-CNT matrices for use as a supercapacitor for an energy storage device were demonstrated. In its present form performance of PMMA-CNT matrices are not optimized for high-performance supercapacitors but may be more suitable for biocompatible component for our SGS system. There are several ways to enhance the performance of these supercapacitors.

\subsection{Future Work}

For BFCs based on PMMA-CNT matrices a number of things can be done in the future to enhance its performance. First issue to be tackle is better immobilization of enzymes through more effective functionalization techniques. This will lead to more enzyme loading, enzyme activity, more active sites and improved performance. The performance of BFCs can also be optimized further by tuning the operational temperature and $\mathrm{pH}$ of the PBS electrolyte [15]. Similarly a better electrolyte with higher ionic conduction and biocompatible with enzymes can be used to enhance the performance $[15,75]$.

Performance of supercapacitor based on PMMA-CNT matrices can be improved in several ways. Better electrolyte instead of PBS such as $\mathrm{KOH}$ can be used [55]. Leakage currents can be reduced through better sealing, better separator such as [74] can be used instead of Whatman filter paper, and contact resistances can be reduced. Finally, the surface of PMMA can be made more porous by treatment and 
finally composite of PMMA and other polymers such as PANI, PDMS, PPy etc may improve the specific capacitance.

\subsection{References}

1. K.S. Cole. In membranes, ions and impulses, University of California Press, 1972

2. M. C. Potter. Electrical effects accompanying the decomposition of organic compounds, Proc. R. Soc. (Lond.), 1911, B84, 260.

3. B. Cohen. The bacterial culture as an electrical half-cell, J. Bacteriol, 1931, 21, 18

4. F. Davis, S. P. J. Higson. Biofuel cells-Recent advances and applications, Biosensors and Bioelectronics, 2007, 22, 1224.

5. R. A. Bullen, T. C. Arnot, J. B. Lakeman, F. C. Walsh. Biofuel cells and their development, Biosensors and Bioelectronics, 2006, 21, 2015.

6. A. T. Yahiro, S. M. Lee, D. O. Kimble. Bioelectrochemistry I, Enzyme utilizing biofuel cell studies, Biochim Biophys Acta, 1964, 88, 375.

7. W. J. Aston, A. P. F. Turner. Biosensors and biofuel cells, Biotechnol Genet Eng Rev, 1984, 1, 89.

8. G. Govil, A. Saran. Biochemical fuel cells, J Indian Chem Soc, 1982, 59, 1226. 
9. J. O. M. Bockris, S. Srinivasan. Fuel cells: their electrochemistry. New York 7 McGraw-Hill. 1969.

10. G. T. R. Palmore, G. M. Whitesides. Microbial and enzymic biofuel cells. ACS Symp Ser, 1994, 566, 271.

11. E. Katz, I. Willner. A biofuel cell with electrochemically switchable and tunable power output. J Am Chem Soc, 2003a, 125, 6803.

12. S. C. Barton, J. Gallaway, P. Atanassov. Enzymatic biofuel cells for implantable and microscale devices, Chem Rev, 2004,104, 4867.

13. D. Ivnitski, B. Branch, P. Atanassov, C. Apblett. Glucose oxidase anode for biofuell cell based on direct electron transfer, Electrochemistry Communications, 2006, 8, 1204

14. Y. Yan, W. Zheng, L. Su, L. Mao. Carbon-nanotube-based glucose $/ \mathrm{O}_{2}$ biofuel cells, Advanced Materials, 2006, 18, 2639.

15. Y. Yan, L. Su, L. Mao. Multi-walled carbon nanotube-based glucose $/ \mathrm{O}_{2}$ biofuel Cell with glucose oxidase and laccase as biocatalyst, Journal of Nanoscience and Nanotechnology, 2007, 7, 1625

16. X. Li, H. Zhou, P. Yu, L. Su, T. Ohsaka, L. Mao. A miniature glucose $/ \mathrm{O}_{2}$ biofuel cell with single-walled carbon nanotubes-modified carbon fiber 
microelectrodes as the substrate, Electrochemistry Communications, 2008, 10, 851

17. F. Gao, L. Viry, M. Maugey, P. Poulin, N. Mano. Engineering hybrid nanotube wires for high-power biofuel cells, Nature Communications, 2010, 1, 2.

18. A. L. Ghindilis, P. Atanasov, E. Wilkins. Enzyme-catalyzed direct electron transfer: fundamentals and analytical applications, Electroanalysis, 1997, 9, 661.

19. W. Schuhmann. Amperometric enzyme biosensors based on optimized electron-transfer pathways and non-manual immobilization procedures. Rev Mol Biotechnol, 2002, 82, 425.

20. R. S. Freire, C. A. Pessoa, L. D. Mello, L. T. Kubota. Direct electron transfer: an approach for electrochemical biosensors with higher selectivity and sensitivity, J Braz Chem Soc, 2003, 14, 230.

21. A. I. Yaropolov, T. K. Sukhomlin, A. A. Karyakin, S. D. Varfolomeev, I. V. Berezin. Possibility of electron tunneling transfer during enzymic catalysis of electrode processes, Dokl Akad Nauk SSSR, 1981, 260, 1192.

22. J. Kim, H. Jia, P. Wang. Challenges in biocatalysis for enzyme-based biofuel cells, Biotechnology Advances, 2006, 24, 296. 
23. E. Katz, B. Filanovsky, I. Willner. A biofuel cell based on two immiscible solvents and glucose oxidase and microperoxidase- 11 monolayerfunctionalized electrodes. New J Chem, 1999, 23, 481.

24. M. R. Tarasevich, V. A. Bogdanovskaya, N. M. Zagudaeva, A. V. Kapustin. Composite materials for direct bioelectrocatalysis of the hydrogen and oxygen reactions in biofuel cells, Russ J Electrochem, 2002, 38, 335.

25. S. D. Minteer, N. L. Akers, C. M. Moore. Enzyme immobilization for use in biofuel cells and sensors, U.S. Pat. Application Publication (2004) US 2004101741.

26. I. Willner, E. Katz, F. Patolsky, A. F. Buckmann. Biofuel cell based on glucose oxidase and microperoxidase-11 monolayer-functionalized electrodes, J Chem Soc, Perkin Transact, Phys Org Chem, 1998, 2, 1817.

27. Kang C, Shin H, Zhang Y, Heller A. Deactivation of bilirubin oxidase by a product of the reaction of urate and $\mathrm{O}_{2}$, Bioelectrochemistry, 2004, 65, 83 .

28. R. Winder. Alcoholic fuel. Chem Ind, 2003, 15, 8.

29. H. Claus. Laccases: structure, reactions, distribution, Micron, 2004, 35, 93.

30. J. A. Cracknell, K. A. Vincent, F. A. Armstrong. Enzymes as working or inspirational electrocatalysts for fuel cells and electrolysis, Chem. Rev., 2008, 108 (7), 2439 
31. Y. Lin, F. Lu, Y. Tu, Z. Ren. Glucose biosensors based on carbon nanotube nanoelectrode ensembles, Nanoletters, 2004, 4, 191.

32. R. O’Hayre, S. K. Cha, W. Colella, F. B. Prinz, Fuel cell fundamentals, John Wiley \& Sons, New York, 2006.

33. B. E. Conway, Electrochemical sSupercapacitors: Scientific fundamentals and technological applications. New York, Kluwer-Plenum, 1999.

34. A. Burke. Ultracapacitors: Why, how, and where is the technology. Journal of Power Sources, 2000, 91, 37.

35. R. Kotz, M. Carlen. Principles and applications of electrochemical capacitors. Electrochimica Acta, 2000, 45, 2483.

36. A. S. Aricò, P. Bruce, B. Scrosati, J. M. Tarascon, W. V. Schalkwijk. Nanostructured materials for advanced energy conversion and storage devices, Nature Materials, 2005, 4, 366.

37. A. Chu, P. Braatz. Comparison of commercial supercapacitors and high power lithium-ion batteries for power-assist applications in hybrid electric vehicles I. Initial characterization, Journal of Power Sources, 2002, 112, 236.

38. L. F. Nazar, G. Goward, F. Leroux, M. Duncan, H. Huang, T. Kerr, J. Gaubicher. Nanostructured materials for energy storage, Inernational Journal of Inorganic Materials, 2001, 3, 191. 
39. H. Pan, J. Li, Y. P. Feng. Carbon nanotubes for supercapacitor, Nanoscale Res Lett, 2010, 5, 654 .

40. Q. L. Feng, D. A. Evans, S. L. Roberson, J. P. Zheng. Ruthenium Oxide Film Electrodes prepared at low temperature for electrochemical capacitors, J. Electrochem Soc, 2001, 148, A833.

41. J. Jiang, A. Kucernak. Electrochemical supercapacitor material based on manganese oxide: Preparation and characterization, Electrochim. Acta, 2002, 47, 2381.

42. M. Mastragostino, C. Arbizzani, F. Soavi. Conducting polymers as electrode materials in supercapacitors, Solid State Ionics, 2002, 148, 493.

43. D. Lozano-Castello, D. Cazorla-Amorós, A. Linares-Solano, S. Shiraishi, H. Kurihara, A. Oya. Influence of Pore Structure and Surface Chemistry on Electric Double Layer Capacitance in Non-aqueous Electrolyte, Carbon, 2003, $41,1765$.

44. E. Frackowiak, K. Jurewicz, K. Szostak, S. Delpeux, F. Béguin. Nanotubular materials as electrodes for supercapacitors, Fuel Process. Technol., 2002, 213, 77.

45. E. Frackowiak. Supercapacitor electrodes from multiwalled carbon nanotubes. Appl. Phys. Lett., 2000, 77, 2421. 
46. E. Frackowiak, F. Beguin. Electrochemical storage of energy in carbon nanotubes and nanostructured, Carbon, 2002, 40, 1775.

47. M. Jayalakshmi and K. Balasubramanian. Simple capacitors to supercapacitors- An overview, Int. J. Electrochem. Sci., 2008, 3, 1196.

48. C. Liu, A.J. Bard, F. Wudl, I. Weitz, J. R. Heath. Electrochemical characterization of films of single walled carbon nanotubes and their possible application in supercapacitors, Electrochem Solid State Lett, 1999, 2, 577.

49. E. Frackowiak, K. Jurewicz, S. Delpeux, F. Beguin. Supercapacitors based on conducting polymers/nanotubes composites, J. Power Sources, 2001, 97, 822.

50. C. Niu, E. K. Sichel, R. Hoch, D. Moy, H. Tennent. High power electrochemical capacitors based on carbon nanotube electrodes, Appl Phys Lett, 1997, 70, 1480.

51. G. L. Che, B. B. Lakshmi, E. R. Fisher, C. R. Martin. Carbon nanotubule membranes for electrochemical energy storage and production, Nature, 1998, $393,346$.

52. J. H. Chen, W. Z. Li, D. Z. Wang, S. X. Yang, J. G. Wen and Z. F. Ren. Electrochemical characterization of carbon nanotubes as electrode in electrochemical double-layer capacitors, Carbon, 2002, 40, 1193. 
53. B. J. Yoon, S. H. Jeong, K. H. Lee, H. S. Kim, C. G. Park and J. H. Han. Electrical properties of electrical double layer capacitors with integrated carbon nanotube electrodes, Chem. Phys. Lett., 2004, 388, 170.

54. Q. Jiang, Y. Zhao, X. Y. Lu, X. T. Zhu, G. Q. Yang, L. J. Song, Y. D. Cai, X. M. Ren, L. Qian. Effects of activation conditions on the electrochemical capacitance of activated carbon nanotubes, Chem. Phys. Lett., 2005, 410, 307.

55. C. Du, J. Yeh, N. Pan. High power density supercapacitors using locally aligned carbon nanotube electrodes, Nanotechnology, 2005, 16, 350.

56. Y. J. Kim, Y. A. Kim, T. Chino, H. Suezaki, M. Endo, M. S. Dresselhaus. Chemically modified multiwalled carbon nanotubes as an additive for supercapacitors, Small, 2006, 2, 339.

57. W. C. Fang, M.S. Leu, K.H. Chen, L.C. Chen. Ultrafast Charging-Discharging Capacitive Property of $\mathrm{RuO} 2$ Nanoparticles on carbon nanotubes using nitrogen incorporation, J. Electrochem. Soc., 2008, 155, K15.

58. Y. Shan, L. Gao. Formation and characterization of multi-walled carbon nanotubes $/ \mathrm{CO}_{3} \mathrm{O}_{4}$ nanocomposites for supercapacitors, Mater. Chem. Phys., $2007103,206$.

59. V. Subramanian, H. Zhu, B. Wei. Synthesis and electrochemical characterizations of amorphous manganese oxide and single walled carbon 
nanotube composites as supercapacitor electrode materials, Electrochem. Commun., 2006 8, 827.

60. Y. G. Wang, L. Yu, Y.Y. Xia. Electrochemical capacitance performance of hybrid supercapacitors based on $\mathrm{Ni}(\mathrm{OH})_{2} /$ carbon nanotube composites and activated carbon, J. Electrochem. Soc., 2006, 153, A743.

61. G. Wang, M. Qu, Z. Yu, R. Yuan. $\mathrm{LiNi}_{0.8} \mathrm{CO}_{0.2} \mathrm{O}_{2} / \mathrm{MWCNT}$ composite electrodes for supercapacitors, Mater. Chem. Phys., 2007, 105, 169.

62. A. L. M. Reddy, S. Ramaprabhu. Nanocrystalline metal oxides dispersed multiwalled carbon nanotubes as supercapacitor electrodes, J. Phys. Chem. C Nanomater. Interfaces, 2007, 111, 7727.

63. K. Lota, V. Khomenko, E. J. Frackowiak. Capacitanvce properties of poly(3,4ethylenedioxythiophene)/carbon nanotubes composites, J. Phys. Chem. Solids, 2004, 65, 295.

64. F. Beguin, K. Szostak, G. Lota, E. Frackowiak. A self supporting electrolyte for supercapacitors prepared by one-step pyrolysis of carbon nanotubes polyacrylonitrile blends, Adv. Mater., 2005, 17, 2380.

65. B. Dong, B. L. He, C. L. Xu, H. L. Li. Preparation and electrochemical characterization of polyaniline/multi-walled carbon nanotubes composites for supercapacitor, Mater. Sci. Eng. B, 2007, 143, 7. 
66. H. Mi, X. Zhang, S. An, X. Ye, S. Yang. Microwave-assisted synthesis and electrochemical capacitance of polyaniline/multi-wall carbon nanotubes composite, Electrochem. Commun., 2007, 9, 2859.

67. L. B. Kong, J. Zhang, J. J. An, Y. C. Luo, L. Kang. MWNTs/PANI composite materials prepared by in-situ chemical oxidative polymerization for supercapacitor electrode, J. Mater. Sci., 2008, 43, 3664.

68. V. Gupta, N. Miura. Polyaniline/single-wall carbon nanotubes (PANI/SWCNT) composites for high performance supercapacitors, Electrochim. Acta, 2006, 52, 1721.

69. Y. K. Zhou, B. L. He, W. J. Zhou, H. L. Li. Preparation and electrochemistry of SWNT/PANI composite films for electrochemical capacitor, J. Electrochem. Soc., 2004, 151, A1052.

70. M. Hughes, G. Z. Chen, M. S. P. Shaffer, D. J. Fray, A. H. Windle. Electrochemical capacitance of a nanoporous composite of carbon nanotubes and polypyrrole, Chem. Mater., 2002, 14, 1610.

71. K. H. An, K. K. Jeon, J. K. Heo, S. C. Lim, D. J. Bae, Y. H. Lee. Highcapacitance supercapacitor using a nanocomposite electrode of single-walled carbon nanotubes and polypyrrole, J. Electrochem. Soc, 2002, 149, A1058. 
72. V. Khomenko, E. Frackowiak, F. Beguin. Determination of the specific capacitance of conducting polymer/nanotubes composite electrodes using different cell configurations, Electrochim. Acta, 2005, 50, 2499.

73. V. Khomenko, E. Frackowiak, K. Jurewics, K. Lota, F. Beguin. Supercapacitors based on conducting polymers/nanotubes composites, J. Power Sources, 2006, 153, 413.

74. A. Arepalli, H. Fireman, C. Huffman, P. Moloney, P. Nikolaev, L. Yowell, C. D. Higgins, K. Kim, P. A. Kohl, S. P. Turano, W. J. Ready. Carbon-nanotubebased electrochemical double-layer capacitor technologies for spaceflight applications, JOM Journal of the Minerals, Metals and Materials Society, 2005, 57, 26.

75. F. Barrière, P. kavanagh, D. Leech. A laccase-glucose oxidase biofuel cell prototype operating in a physiological buffer, Electrochemical Acta, 2006, 51, 5187. 


\section{Chapter 6}

\section{Future work on energy SGS system based on PMMA-CNT matrices}

This thesis focused on investigating the performances of PMMA-CNT matrices for application in vacuum electronics (electron field emission, Chapter 3), glucose sensing (Chapter 4), biofuel cells (BFCs) and supercapacitors (Chapter 5). Future works these applications were proposed at the end these chapters. In chapter 5 we proposed for an energy sensing, generation and storage (SGS) system that integrate glucose biosensors, BFCs and supercapacitors. Such a SGS system may be implantable and help, to some extents, in controlling the glucose level, especially patients suffering from type-I diabetes which requires more stringent monitoring and control of blood glucose level

This chapter provides some evaluations on possible design of the SGS system. The major challenge lies ahead in integrating glucose sensors, BFCs and supercapacitors to form the SGS system. The working principle of proposed system is described as follows. The glucose sensor continuously monitors the blood glucose level. When it senses excess glucose level it will activate the biofuel cells to convert the excessive glucose into energy. The generated energy is then stored in the supercapacitor since it can charge and discharge quickly. The block diagram of the SGS system is presented in Figure 6.1. 
Apart from the three components, other signal processing and control units will also need to be integrated into the SGS system for regulating the function of each component. In addition, each component in the SGS system will need to have fluidic channels that allow body fluid such as blood to flow through them. Finally the whole SGS system will need to be encapsulated in a biocompatible polymer material to prevent the buildup of tissue around it once implanted.

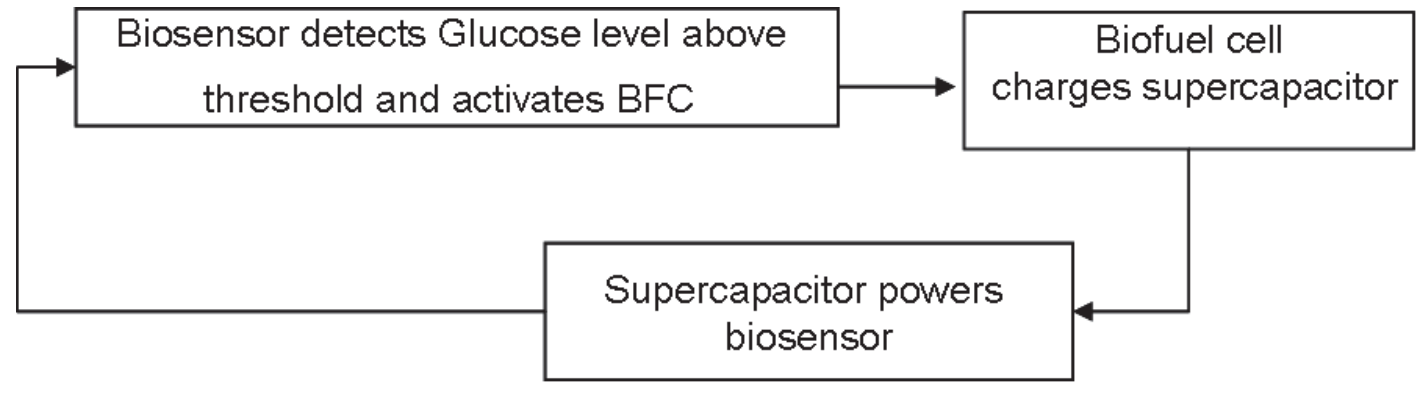

Figure 6.1: Circuit block diagram for SGS

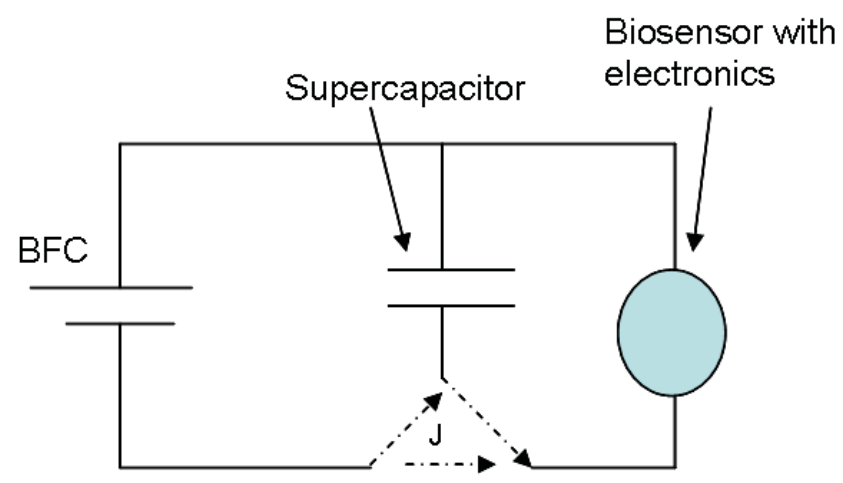

Figure 6.2: Circuit component diagram for charging and discharging of SGS device showing various components: BFC, supercapacitor, biosensor with low power electronics to monitor current flow through junction " $j$ ". 
Here, we outline the possible arrangement of the circuit components in Figure 6.2. In principle, we can use the supercapacitor to power the biosensor during the glucose monitoring state. Here, current will flow from the charged supercapacitor to the biosensor at junction "J" as controlled by the electronics of the control units. When the biosensor detects glucose level above threshold, it will order the electronics to modify the connections at junction " $J$ " to connect BFC to the supercapacitor. Thereafter, the BFCs will start to generate energy and store the generated current to the supercapacitor, while the biosensor continues in the monitoring state. When the glucose level falls below the threshold or when the supercapacitor is fully charged, the electronics will disconnect the BFCs and the supercapacitor, while maintaining the monitoring state. In another scenario when supercapacitor is depleted of charge and unable to power biosensor then the electronics will initiate an alarm so that more foods/glucose be taken. It can be seen already that electronics will play a major role in modulating the current paths at junction " $\mathrm{J}$ ".

Future work will thus be focused on designing a highly efficient and low powered electronic control unit to regulate and control the current flow through the junction "J". Combination of such control unit with the sensing, generation and storage components will lead to a working SGS device. It goes without saying that SGS can not only power biosensor but modifications to this device can be used to power heart pacemakers and other implants. 


\section{APPENDIX-A}

\section{Appendix A1: Calculation for Glucose Consumption Rate:}

At first the area from i-t curve (Figure 4.10(a) in Chapter 4) was calculated. Area under curve gives amount of charge.

Calculated Area from i-t curve: 0.1247 Coulomb

$$
\text { Chemical reaction: } \quad \text { glucose } \rightarrow \text { gluconolactone }+2 \mathrm{e}^{-}+2 \mathrm{H}^{+}
$$

Also, Charge on $1 \mathrm{e}^{-}=1.6 \times 10^{-19}$ Coulomb

$0.1247 \mathrm{C}=$ number of electrons $\approx 1 / 2$ number of electrons (as there are two electrons released from consumption of one molecule of glucose as seen in reaction) $\approx$ number of glucose molecule consumed

So the number of electrons $=\frac{0.1247 C}{1.6 \times 10^{-19} C}=7.794 \times 10^{17}$

Therefore, number of glucose molecule consumed $=\frac{7.794 \times 10^{17}}{2}=3.897 \times 10^{17}$

One mole of a substance has $6.023 \times 10^{23}$ molecules.

Number of moles of glucose $=\frac{3.897 \times 10^{17}}{6.023 \times 10^{23}}=0.647 \mu$ moles of glucose

Time taken for consumption from i-t curve $=6.2 \times 10^{4}$ seconds

Hence, glucose consumption Rate $=\frac{0.647 \times 10^{-6}}{6.2 \times 10^{4}}$

$$
=0.104 \times 10^{-10} \mathrm{~mol} / \mathrm{sec} \text { or } 0.0104 \text { nano-mol} / \mathrm{sec}
$$




\section{APPENDIX-B}

\section{Appendix B-1: Calibration curves for different areas}

Linear fitting of Current vs. concentration curves obtained from amperometric response using Au target as counter electrode:
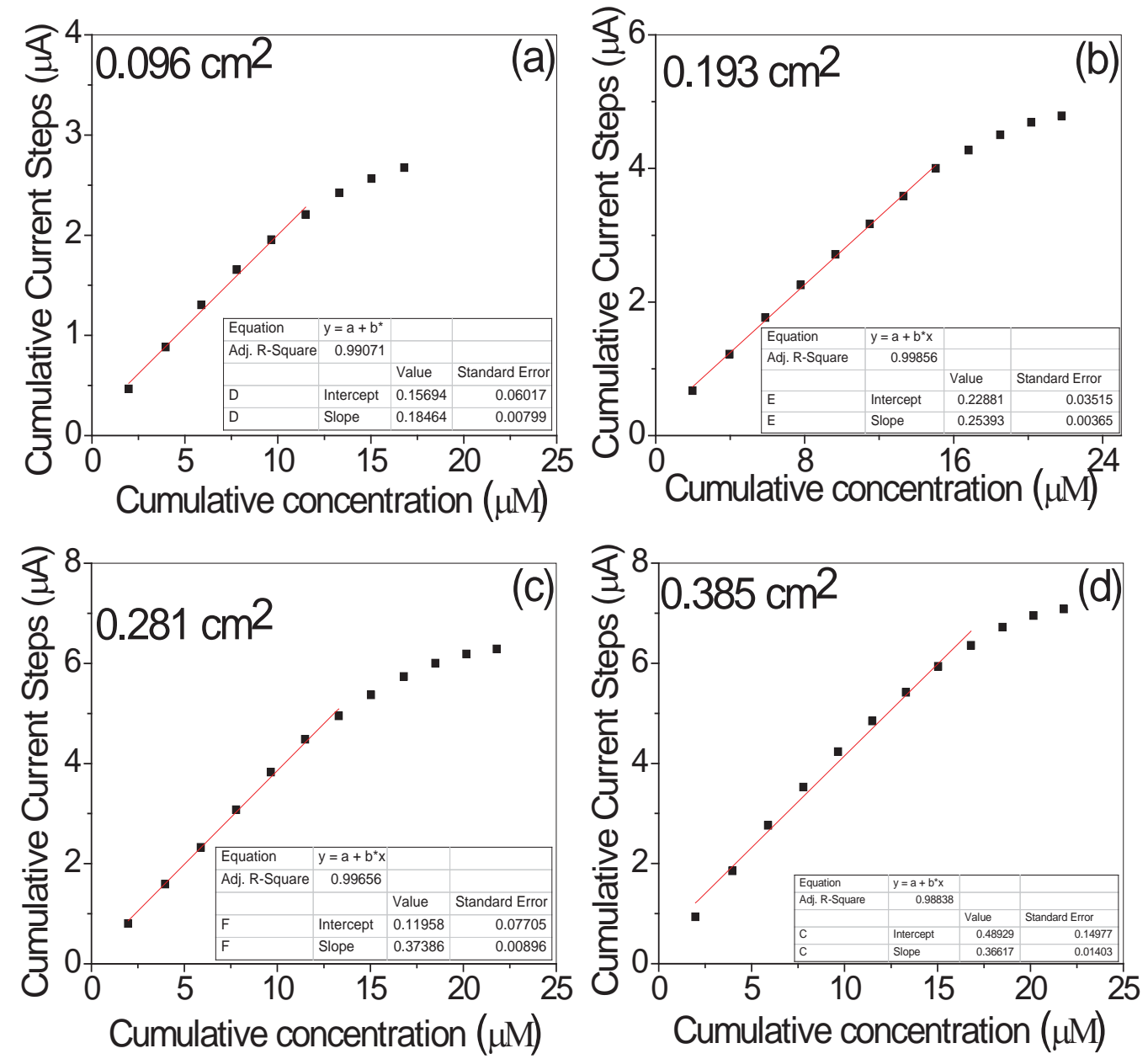

Figure B. 1: Linear fitting of calibration curves of Figure 4.18 in chapter 4 for areas $0.096 \mathrm{~cm}^{2}, 0.193 \mathrm{~cm}^{2}, 0.281 \mathrm{~cm}^{2}$ and $0.385 \mathrm{~cm}^{2}$ 

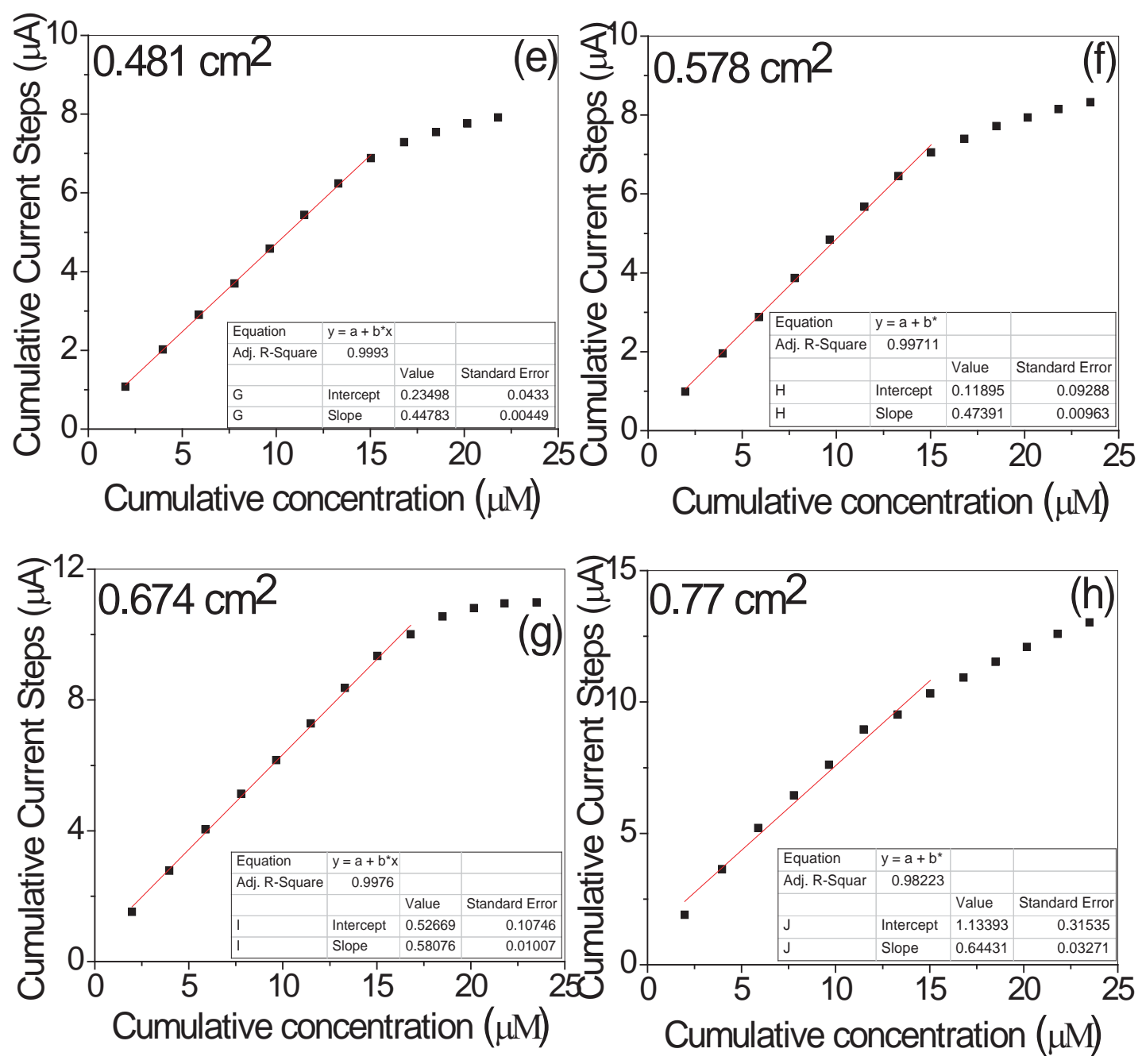

Figure B.2: Linear fitting of calibration curves of Figure 4.18 in chapter 4 for areas $0.481 \mathrm{~cm}^{2}, 0.578 \mathrm{~cm}^{2}, 0.674 \mathrm{~cm}^{2}$ and $0.77 \mathrm{~cm}^{2}$ 
The results shown here is the $\mathrm{CV}$ and amperometric results of biosensor using platinum wire as the counter electrode.
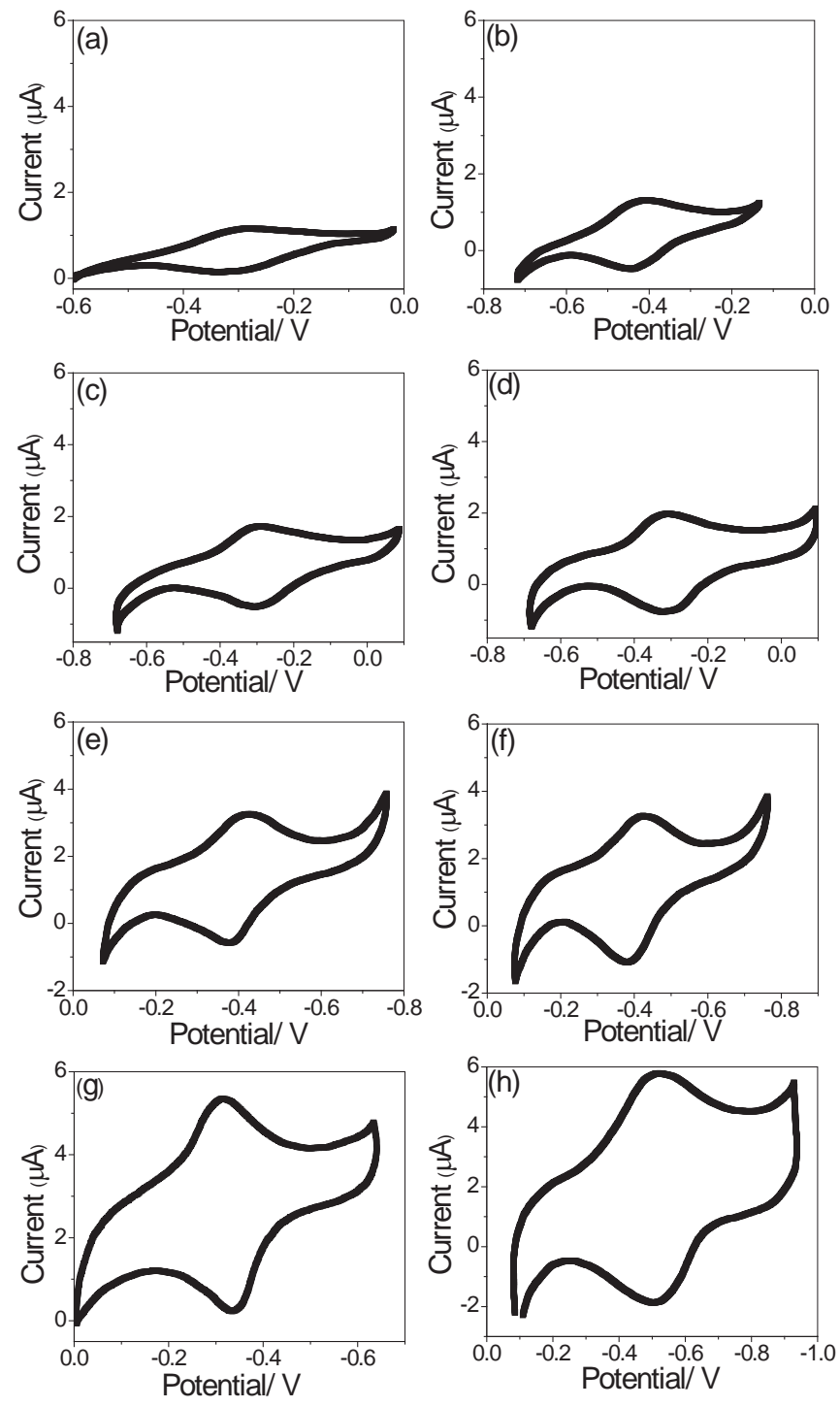

Figure B.3: Cyclic voltammogram of (a-d) GO $O_{x}$-PMMA-CNTs biosensor show two symmetrical peaks with anodic and cathodic peaks for areas of $0.096 \mathrm{~cm}^{2}, 0.193 \mathrm{~cm}^{2}, 0.289$ $\mathrm{cm}^{2}, 0.385 \mathrm{~cm}^{2}, 0.481 \mathrm{~cm}^{2}, 0.578 \mathrm{~cm}^{2}, 0.674 \mathrm{~cm}^{2}$ and $0.77 \mathrm{~cm}^{2}$ respectively at scan rate of $100 \mathrm{mV} / \mathrm{s}$ using platinum wire as the counter electrode. 

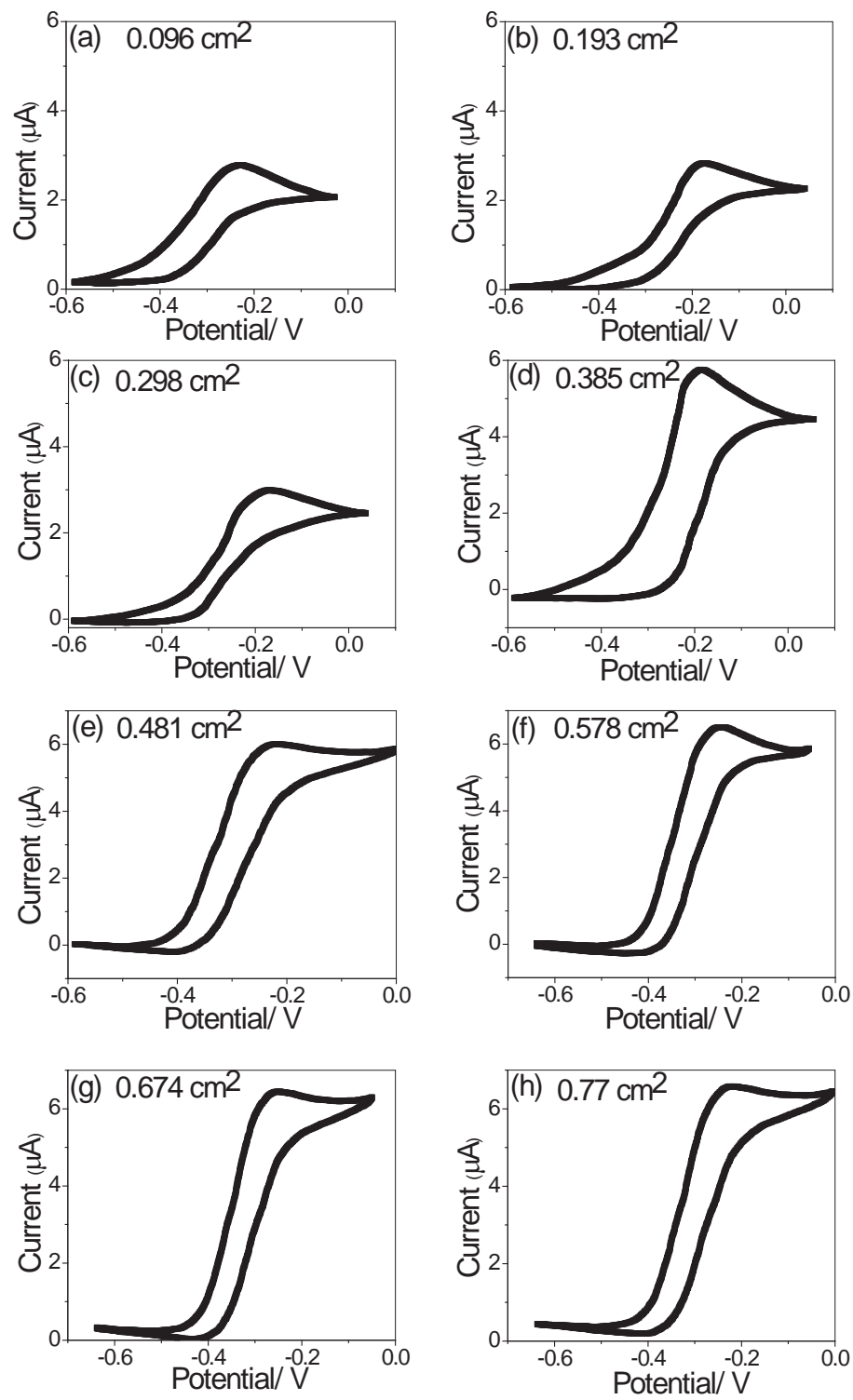

Figure B.4: (a)-(h): Cyclic voltammogram of GOx-PMMA-CNTs biosensor after adding glucose solution for areas of $0.096 \mathrm{~cm}^{2}, 0.193 \mathrm{~cm}^{2}, 0.289 \mathrm{~cm}^{2}, 0.385 \mathrm{~cm}^{2} 0.481 \mathrm{~cm}^{2}, 0.578 \mathrm{~cm}^{2}$, $0.674 \mathrm{~cm}^{2}$ and $0.77 \mathrm{~cm}^{2}$ respectively at scan rate of $100 \mathrm{mV} / \mathrm{s}$ using platinum wire as the counter electrode. 


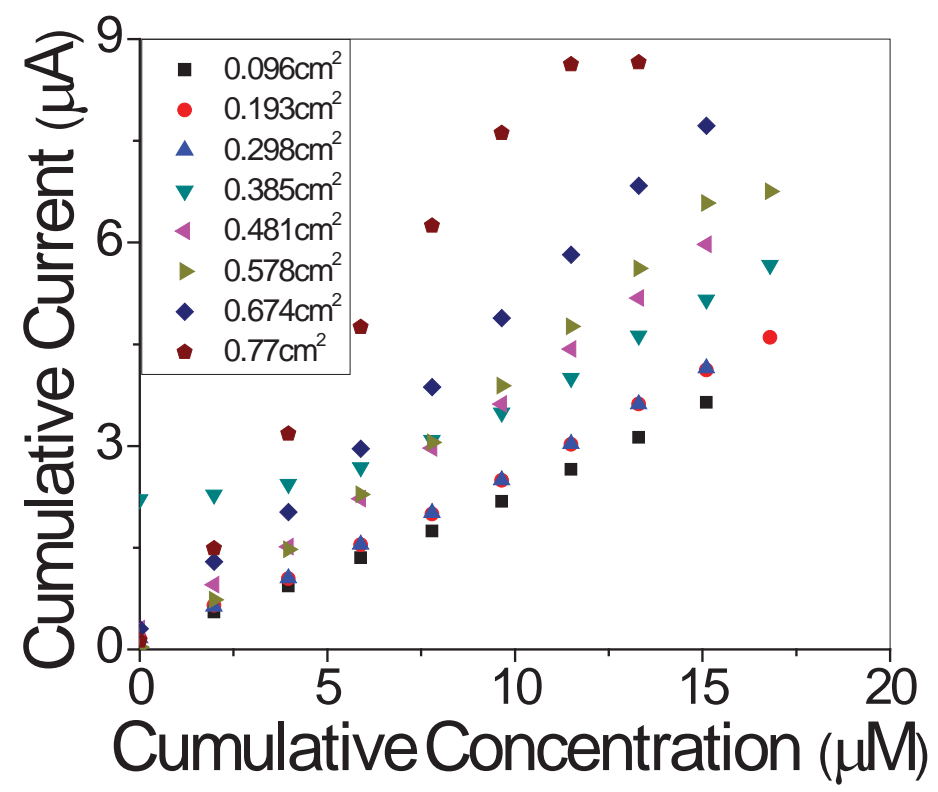

Figure B.5: Calibration curve of Current response vs. Glucose concentration for $G O_{x}-P M M A-$ CNT biosensor for areas $0.096 \mathrm{~cm}^{2}, 0.193 \mathrm{~cm}^{2}, 0.289 \mathrm{~cm}^{2}, 0.385 \mathrm{~cm}^{2} 0.481 \mathrm{~cm}^{2}, 0.578 \mathrm{~cm}^{2}$, $0.674 \mathrm{~cm}^{2}$ and $0.77 \mathrm{~cm}^{2}$ respectively using Pt wire as counter electrode 


\title{
Appendix-C: Permissions
}

\author{
C.1 Benjamin Ulmen, Chapter 1, Reference [28], Figure 1.8 (a)
}

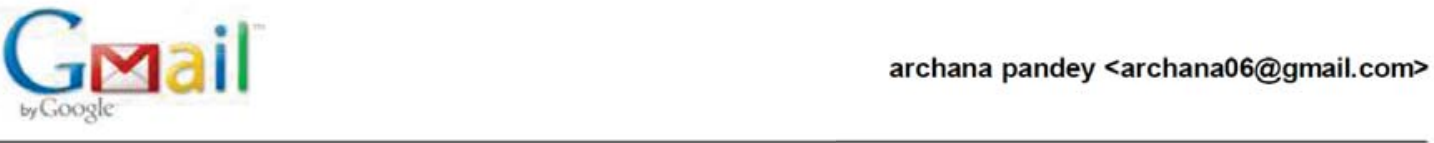

\section{Request for permission}

3 messages

archana pandey <archana06@gmail.com>

Mon, Oct 4, 2010 at 1:56 AM

To: Ben Ulmen <baulmen@gmail.com>, bulmen2@illinois.edu

Dear Ben,

I hope things are going well for you. I am finishing up here and am writing up a chapter on pecvd. I would like to use one of the figures from your Masters Thesis. This figure is schematic diagram of PECVD chamber (Figure 3.1.2: Schematic diagram of the PECVD system). Kindly provide me your permission in writing to use this figure.

Thanks,

Archana

Ben Ulmen <bulmen2@illinois.edu>

Mon, Oct 4, 2010 at 8:28 AM

To: archana pandey <archana06@gmail.com>

Hi Archana,

I give you permission to use any figures from my M.S thesis in your dissertation. Good luck on the writing and

finishing up!

-Ben

[Quoted text hidden]

archana pandey <archana06@gmail.com>

Mon, Oct 4, 2010 at 11:50 AM

To: Ben Ulmen <bulmen2@illinois.edu>

Thanks a lot Ben. I appreciate your help. Actually I wanted to modify it a bit and I'll see if I have enough time to do it. Thanks once again.

Regards,

Archana

[Quoted text hidden] 
C.2 Springer, Chapter 2, Reference [1], Figure 2.2

Gmail

archana pandey <archana06@gmail.com>

\section{Fwd: Request for permission to use Figure from book}

1 message

Archana Pandey <arpandey@mtu.edu>

Tue, Oct 26, 2010 at 1:18 PM

To: archana06 <archana06@gmail.com>

-..-- Forwarded Message -..-.-

From: "Permissions Europe/NL" <Permissions.Dordrecht@springer.com>

To: arpandey@mtu.edu

Sent: Tuesday, October 26, 2010 8:46:34 AM GMT -05:00 US/Canada Eastern

Subject: RE: Request for permission to use Figure from book

Dear Sir,

With reference to your request (copy herewith) to reprint material on which Springer Science and Business Media controls the copyright, our permission is granted, free of charge, for the use indicated in your enquiry.

This permission

- $\quad$ allows you non-exclusive reproduction rights throughout the World.

- $\quad$ permission includes use in an electronic form, provided that content is

* password protected;

* at intranet;

- excludes use in any other electronic form. Should you have a specific project in mind, please reapply for permission.

- requires a full credit (Springer/Kluwer Academic Publishers book/journal title, volume, year of publication, page, chapter/article title, name(s) of author(s), figure number(s), original copyright notice) to the publication in which the material was originally published, by adding: with kind permission of Springer Science and Business Media.

The material can only be used for the purpose of defending your dissertation, and with a maximum of 100 extra copies in paper.

Permission free of charge on this occasion does not prejudice any rights we might have to charge for reproduction of our copyrighted material in the future.

Best regards,

Nel van der Werf (Ms)

Rights and Permissions/Springer

Van Godewijckstraat 30 | P.O. Box 17

3300 AA Dordrecht | The Netherlands

tel $+31(0) 786576298$

fax +31 (0)78 65 76-300

Nel.vanderwerf @springer.com

www.springer.com

-----Original Message----.

From: SpringerAlerts@springeronline.com [mailto:SpringerAlerts@springeronline.com]

Sent: Wednesday, October 20, 2010 8:26 AM

To: Permissions Europe/NL

Subject: Request for permission to use Figure from book 


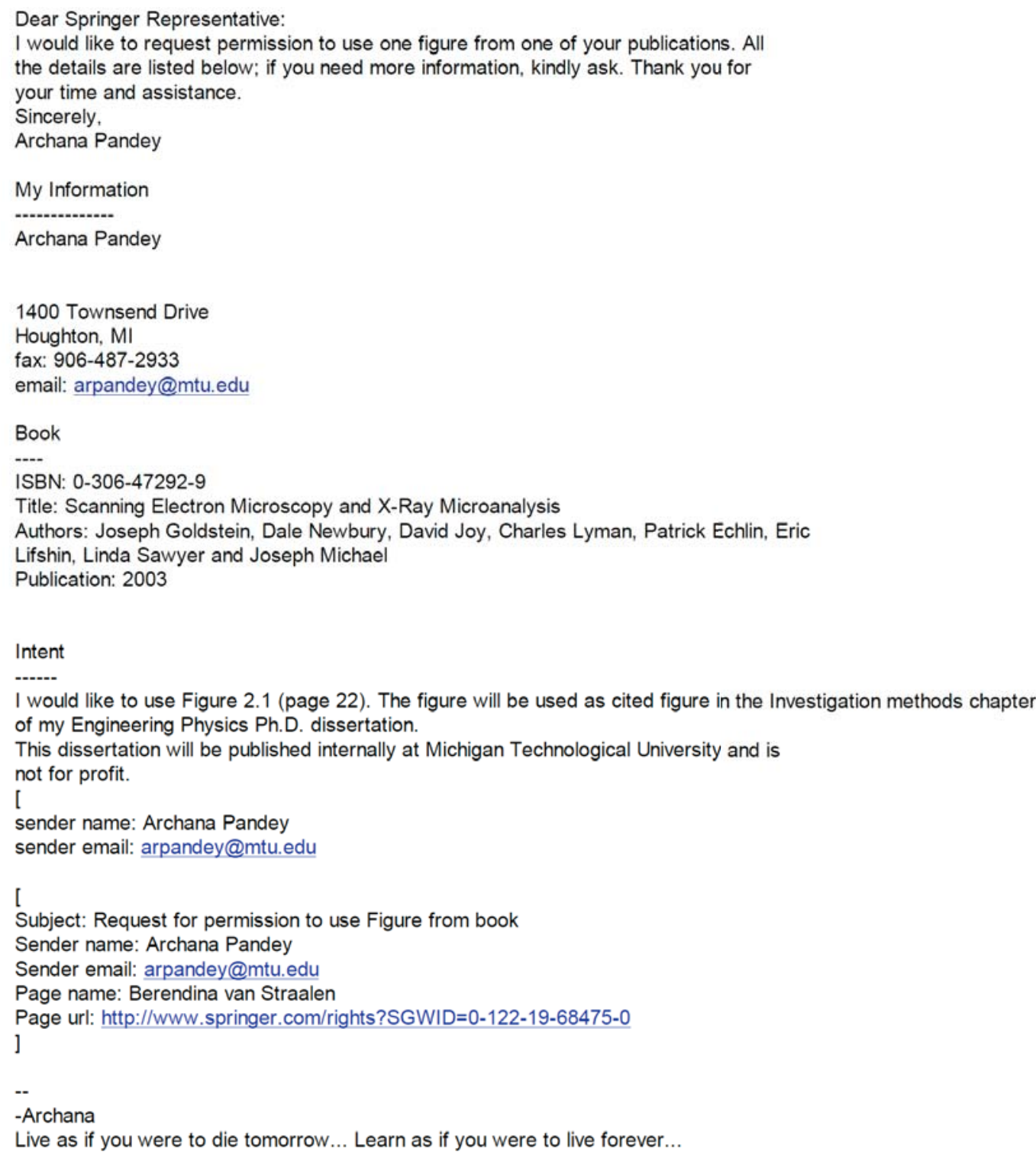




\title{
C.3 Springer, Chapter 2, Reference [3], Figure 2.4
}

archana pandey <archana06@gmail.com>

\section{Fwd: request for permission}

\author{
1 message
}

Archana Pandey <arpandey@mtu.edu>

Mon, Oct 25, 2010 at 5:44 PM

To: archana06 <archana06@gmail.com>

-...- Forwarded Message -.....

From: "Permissions Europe/NL" < Permissions.Dordrecht@springer.com>

To: arpandey@mtu.edu

Sent: Monday, October 25, 2010 2:12:08 AM GMT -05:00 US/Canada Eastern

Subject: RE: request for permission

Dear mr. Pandey,

With reference to your request (copy herewith) to reprint material on which Springer Science and Business Media controls the copyright, our permission is granted, free of charge, for the use indicated in your enquiry.

This permission

- allows you non-exclusive reproduction rights throughout the World.

- permission includes use in an electronic form, provided that content is

" password protected;

* at intranet:

- excludes use in any other electronic form. Should you have a specific project in mind, please reapply for permission.

- requires a full credit (Springer/Kluwer Academic Publishers book/journal title, volume, year of publication, page, chapter/article title, name(s) of author(s), figure number(s), original copyright notice) to the publication in which the material was originally published, by adding: with kind permission of Springer Science and Business Media.

* The material can only be used for the purpose of defending your dissertation, and with a maximum of 100 extra copies in paper.

Permission free of charge on this occasion does not prejudice any rights we might have to charge for reproduction of our copyrighted material in the future.

Berendina van Straalen

Springer

Head of Rights and Permissions

Special Licensing Department

Van Godewijckstraat 30 | 3311 GX Dordrecht

P.O.Box 17 | 3300 AA Dordrecht

The Netherlands

Tel. +31786576210

Fax +31786576300

www.springer.com

2010 FAIRS:

6-10 October - Frankfurt

30 November - 2 December - London Online

Book fairs - permissions.bookfairs@springer.com

Translation rights - translations.dordrecht@springer.com

offers: http://www.springer.com/rights?SGWID=0-122-0-0-0

Special Licensing - TPL.dordrecht@springer.com

Permissions - permissions.dordrecht@springer.com 


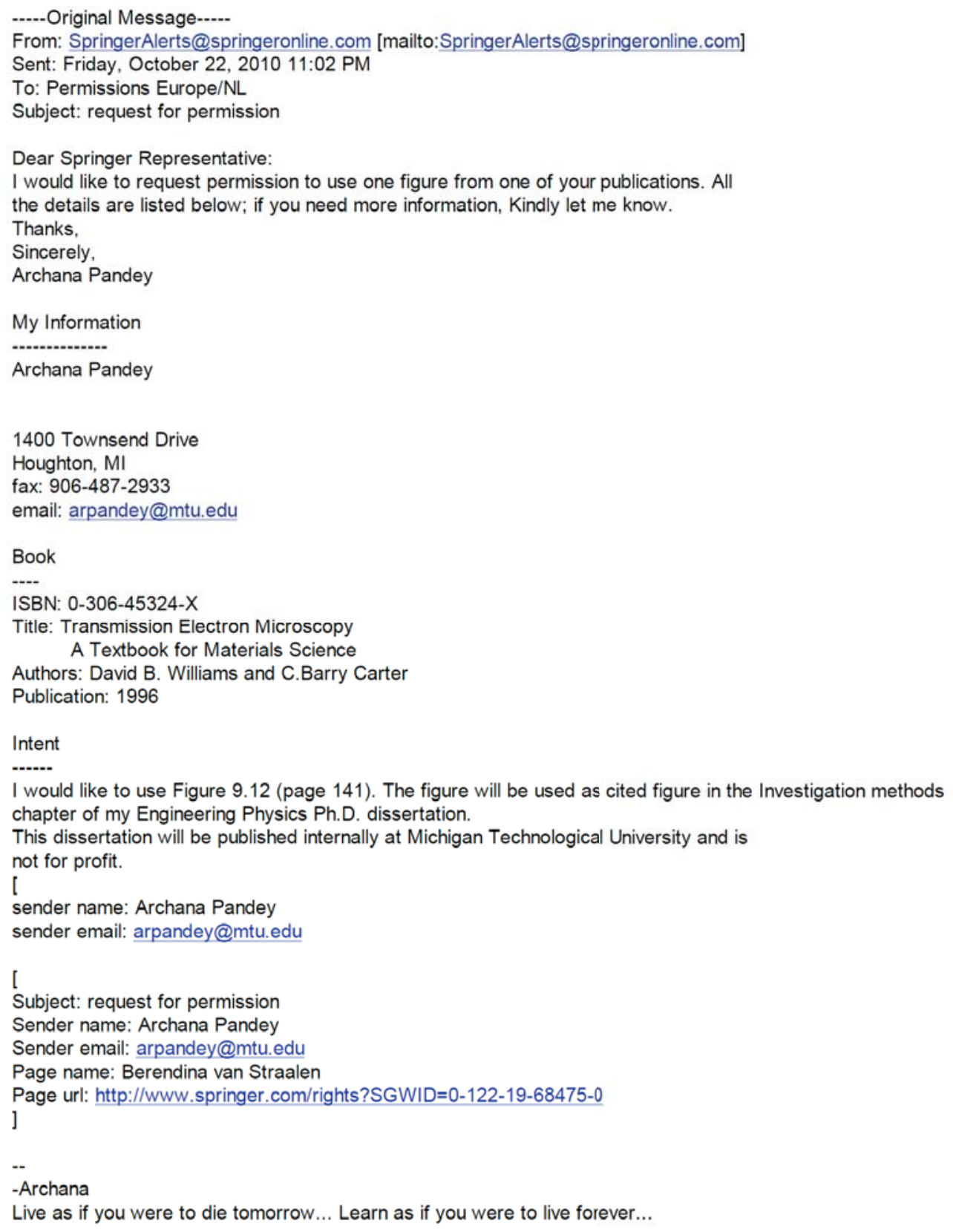




\title{
C.4.Permission from Elsevier for figures 3.5, 3.6, 3.7, and 3.8 in Chapter 3 , Reference [16].
}

\author{
ELSEVIER LICENSE \\ TERMS AND CONDITIONS
}

Oct 20, 2010

This is a License Agreement between Archana Pandey ("You") and Elsevier ("Elsevier") provided by Copyright Clearance Center ("CCC"). The license consists of your order details, the terms and conditions provided by Elsevier, and the payment terms and conditions.

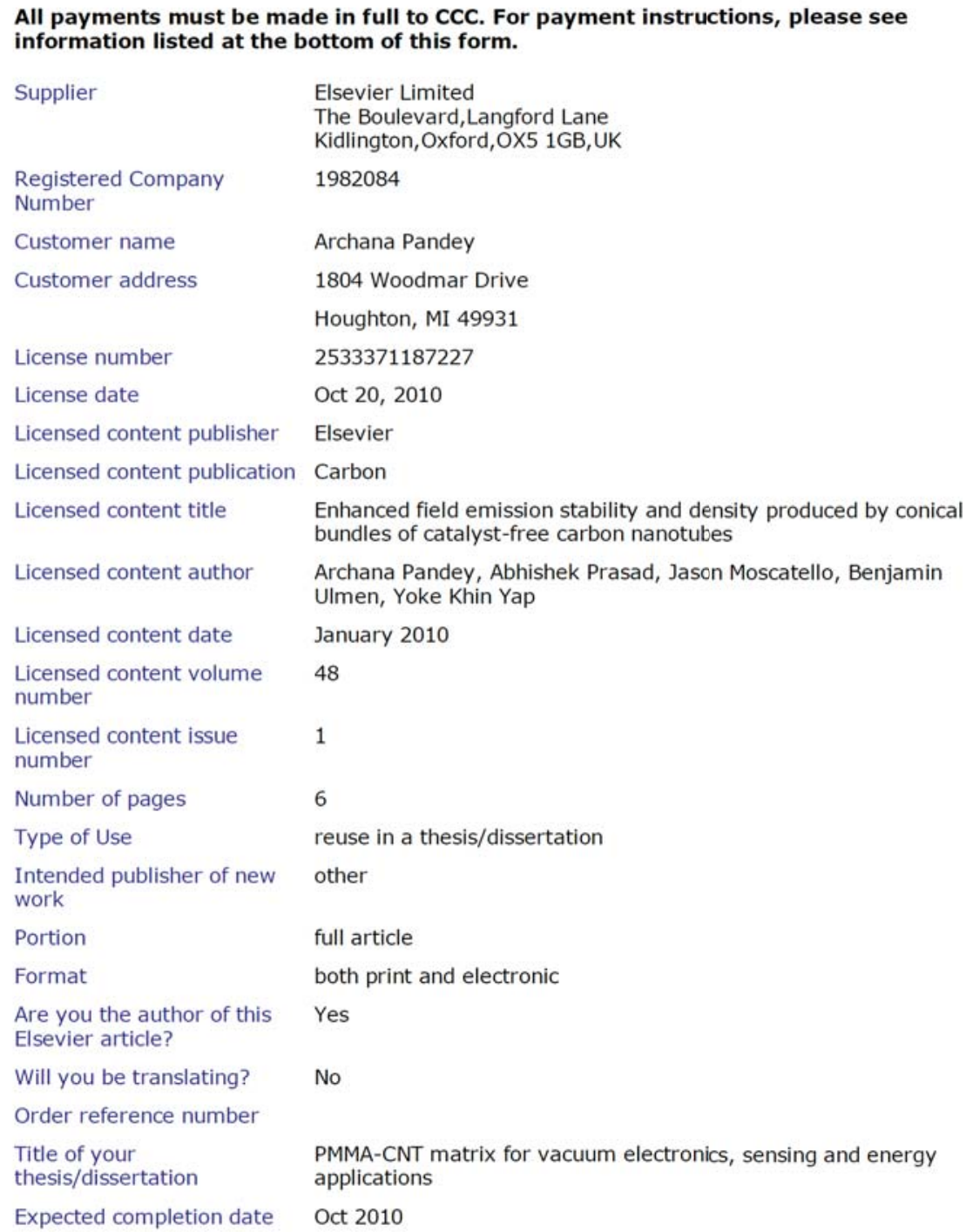

Expected completion date Oct 2010 
Estimated size (number of 200
pages)

Elsevier VAT number

Terms and Conditions

GB 494627212

\section{INTRODUCTION}

1. The publisher for this copyrighted material is Elsevier. By clicking "accept" in connection with completing this licensing transaction, you agree that the following terms and conditions apply to this transaction (along with the Billing and Payment terms and conditions established by Copyright Clearance Center, Inc. ("CCC"), at the time that you opened your Rightslink account and that are available at any time at http://myaccount.copyright.com).

\section{GENERAL TERMS}

2. Elsevier hereby grants you permission to reproduce the aforementioned material subject to the terms and conditions indicated.

3. Acknowledgement: If any part of the material to be used (for example, figures) has appeared in our publication with credit or acknowledgement to another source, permission must also be sought from that source. If such permission is not obtained then that material may not be included in your publication/copies. Suitable acknowledgement to the source must be made, either as a footnote or in a reference list at the end of your publication, as follows:

"Reprinted from Publication title, Vol /edition number, Author(s), Title of article / title of chapter, Pages No., Copyright (Year), with permission from Elsevier [OR APPLICABLE SOCIETY COPYRIGHT OWNER]." Also Lancet special credit - "Reprinted from The Lancet, Vol. number, Author(s), Title of article, Pages No., Copyright (Year), with permission from Elsevier."

4. Reproduction of this material is confined to the purpose and/or media for which permission is hereby given.

5. Altering/Modifying Material: Not Permitted. However figures and illustrations may be altered/adapted minimally to serve your work. Any other abbreviations, additions, deletions and/or any other alterations shall be made only with prior written authorization of Elsevier Ltd. (Please contact Elsevier at permissions@elsevier.com)

6. If the permission fee for the requested use of our material is waived in this instance, please be advised that your future requests for Elsevier materials may attract a fee.

7. Reservation of Rights: Publisher reserves all rights not specifically granted in the combination of (i) the license details provided by you and accepted in the course of this licensing transaction, (ii) these terms and conditions and (iii) CCC's Billing and Payment terms and conditions.

8. License Contingent Upon Payment: While you may exercise the rights licensed immediately upon issuance of the license at the end of the licensing process for the transaction, provided that you have disclosed complete and accurate details of your proposed use, no license is finally effective unless and until full payment is received from you (either by publisher or by CCC) as provided in CCC's Billing and Payment terms and 
conditions. If full payment is not received on a timely basis, then any license preliminarily granted shall be deemed automatically revoked and shall be void as if never granted. Further, in the event that you breach any of these terms and conditions or any of CCC's Billing and Payment terms and conditions, the license is automatically revoked and shall be void as if never granted. Use of materials as described in a revoked license, as well as any use of the materials beyond the scope of an unrevoked license, may constitute copyright infringement and publisher reserves the right to take any and all action to protect its copyright in the materials.

9. Warranties: Publisher makes no representations or warranties with respect to the licensed material.

10. Indemnity: You hereby indemnify and agree to hold harmless publisher and CCC, and their respective officers, directors, employees and agents, from and against any and all claims arising out of your use of the licensed material other than as specifically authorized pursuant to this license.

11. No Transfer of License: This license is personal to you and may not be sublicensed, assigned, or transferred by you to any other person without publisher's written permission.

12. No Amendment Except in Writing: This license may not be amended except in a writing signed by both parties (or, in the case of publisher, by $\mathrm{CCC}$ on publisher's behalf).

13. Objection to Contrary Terms: Publisher hereby objects to any terms contained in any purchase order, acknowledgment, check endorsement or other writing prepared by you, which terms are inconsistent with these terms and conditions or CCC's Billing and Payment terms and conditions. These terms and conditions, together with CCC's Billing and Payment terms and conditions (which are incorporated herein), comprise the entire agreement between you and publisher (and CCC) concerning this licensing transaction. In the event of any conflict between your obligations established by these terms and conditions and those established by CCC's Billing and Payment terms and conditions, these terms and conditions shall control.

14. Revocation: Elsevier or Copyright Clearance Center may deny the permissions described in this License at their sole discretion, for any reason or no reason, with a full refund payable to you. Notice of such denial will be made using the contact information provided by you. Failure to receive such notice will not alter or invalidate the denial. In no event will Elsevier or Copyright Clearance Center be responsible or liable for any costs, expenses or damage incurred by you as a result of a denial of your permission request, other than a refund of the amount(s) paid by you to Elsevier and/or Copyright Clearance Center for denied permissions.

\section{LIMITED LICENSE}

The following terms and conditions apply only to specific license types:

15. Translation: This permission is granted for non-exclusive world English rights only unless your license was granted for translation rights. If you licensed translation rights you may only translate this content into the languages you requested. A professional translator must perform all translations and reproduce the content word for word preserving the integrity of the article. If this license is to re-use 1 or 2 figures then permission is granted for non-exclusive world rights in all languages. 
16. Website: The following terms and conditions apply to electronic reserve and author websites:

Electronic reserve: If licensed material is to be posted to website, the web site is to be password-protected and made available only to bona fide students registered on a relevant course if:

This license was made in connection with a course,

This permission is granted for 1 year only. You may obtain a license for future website posting,

All content posted to the web site must maintain the copyright information line on the bottom of each image,

A hyper-text must be included to the Homepage of the journal from which you are licensing at http://www.sciencedirect.com/science/journal/xxxxx or the Elsevier homepage for books at http://www.elsevier.com, and

Central Storage: This license does not include permission for a scanned version of the material to be stored in a central repository such as that provided by Heron/XanEdu.

17. Author website for journals with the following additional clauses:

All content posted to the web site must maintain the copyright information line on the bottom of each image, and

he permission granted is limited to the personal version of your paper. You are not allowed to download and post the published electronic version of your article (whether PDF or HTML, proof or final version), nor may you scan the printed edition to create an electronic version,

A hyper-text must be included to the Homepage of the journal from which you are licensing at http://www.sciencedirect.com/science/journal/xxxxx, As part of our normal production process, you will receive an e-mail notice when your article appears on Elsevier's online service ScienceDirect (www.sciencedirect.com). That e-mail will include the article's Digital Object Identifier (DOI). This number provides the electronic link to the published article and should be included in the posting of your personal version. We ask that you wait until you receive this e-mail and have the DOI to do any posting.

Central Storage: This license does not include permission for a scanned version of the material to be stored in a central repository such as that provided by Heron/XanEdu.

18. Author website for books with the following additional clauses:

Authors are permitted to place a brief summary of their work online only.

A hyper-text must be included to the Elsevier homepage at http://www.elsevier.com

All content posted to the web site must maintain the copyright information line on the bottom of each image

You are not allowed to download and post the published electronic version of your chapter, nor may you scan the printed edition to create an electronic version.

Central Storage: This license does not include permission for a scanned version of the material to be stored in a central repository such as that provided by Heron/XanEdu.

19. Website (regular and for author): A hyper-text must be included to the Homepage of the journal from which you are licensing at http://www.sciencedirect.com/science/journal /xxxxx. or for books to the Elsevier homepage at http://www.elsevier.com 
20. Thesis/Dissertation: If your license is for use in a thesis/dissertation your thesis may be submitted to your institution in either print or electronic form. Should your thesis be published commercially, please reapply for permission. These requirements include permission for the Library and Archives of Canada to supply single copies, on demand, of the complete thesis and include permission for UMI to supply single copies, on demand, of the complete thesis. Should your thesis be published commercially, please reapply for permission.

\section{Other Conditions:}

v1.6

Gratis licenses (referencing $\$ 0$ in the Total field) are free. Please retain this printable license for your reference. No payment is required.

If you would like to pay for this license now, please remit this license along with your payment made payable to "COPYRIGHT CLEARANCE CENTER" otherwise you will be invoiced within $\mathbf{4 8}$ hours of the license date. Payment should be in the form of a check or money order referencing your account number and this invoice number

RLNK10869710.

Once you receive your invoice for this order, you may pay your invoice by credit card. Please follow instructions provided at that time.

Make Payment To:

Copyright Clearance Center

Dept 001

P.O. Box 843006

Boston, MA 02284-3006

If you find copyrighted material related to this license will not be used and wish to cancel, please contact us referencing this license number 2533371187227 and noting the reason for cancellation.

Questions? customercare@copyright.com or +1-877-622-5543 (toll free in the US) or +1-978-646-2777. 


\section{C.5 Permission from American Chemical Society for figures 3.3, 3.9, 3.10 and 3.11 in Chapter 3, Reference [28].}

\section{AMERICAN CHEMICAL SOCIETY LICENSE TERMS AND CONDITIONS}

This is a License Agreement between Archana Pandey ("You") and American Chemical Society ("American Chemical Society") provided by Copyright Clearance Center ("CCC"). The license consists of your order details, the terms and conditions provided by American Chemical Society, and the payment terms and conditions.

\begin{tabular}{|c|c|}
\hline \multicolumn{2}{|c|}{$\begin{array}{l}\text { All payments must be made in full to CCC. For payment instructions, please see } \\
\text { information listed at the bottom of this form. }\end{array}$} \\
\hline License Number & 2533370713293 \\
\hline License Date & Oct 20, 2010 \\
\hline Licensed content publisher & American Chemical Society \\
\hline Licensed content publication & ACS Nano \\
\hline Licensed content title & Stable Electron Field Emission from PMMA-CNT Matrices \\
\hline Licensed content author & Archana Pandey et al. \\
\hline Licensed content date & Oct 1,2010 \\
\hline Volume number & 0 \\
\hline Issue number & 0 \\
\hline Type of Use & Thesis/Dissertation \\
\hline Requestor type11 & Not specified \\
\hline Format & Print \\
\hline Portion & Full article \\
\hline Author of this ACS article & Yes \\
\hline \multicolumn{2}{|l|}{ Order reference number } \\
\hline $\begin{array}{l}\text { Title of the thesis / } \\
\text { dissertation }\end{array}$ & $\begin{array}{l}\text { PMMA-CNT matrix for vacuum electronics, sensing and energy } \\
\text { applications }\end{array}$ \\
\hline Expected completion date & Oct 2010 \\
\hline Estimated size(pages) & 200 \\
\hline Billing Type & Invoice \\
\hline \multirow[t]{4}{*}{ Billing Address } & 1804 Woodmar Drive \\
\hline & Apt A \\
\hline & Houghton, MI 49931 \\
\hline & United States \\
\hline \multicolumn{2}{|l|}{ Customer reference info } \\
\hline Total & 0.00 USD \\
\hline
\end{tabular}

\section{Thesis/Dissertation}




\section{ACS / RIGHTSLINK TERMS \& CONDITIONS THESIS/DISSERTATION}

\section{INTRODUCTION}

The publisher for this copyrighted material is the American Chemical Society. By clicking "accept" in connection with completing this licensing transaction, you agree that the following terms and conditions apply to this transaction (along with the Billing and Payment terms and conditions established by Copyright Clearance Center, Inc. ("CCC"), at the time that you opened your Rightslink account and that are available at any time at $<$ http://myaccount.copyright.com $>$ ).

\section{LIMITED LICENSE}

Publisher hereby grants to you a non-exclusive license to use this material. Licenses are for one-time use only with a maximum distribution equal to the number that you identified in the licensing process; any form of republication must be completed within 60 days from the date hereof (although copies prepared before then may be distributed thereafter).

\section{GEOGRAPHIC RIGHTS: SCOPE}

Licenses may be exercised anywhere in the world.

\section{RESERVATION OF RIGHTS}

Publisher reserves all rights not specifically granted in the combination of (i) the license details provided by you and accepted in the course of this licensing transaction, (ii) these terms and conditions and (iii) CCC's Billing and Payment terms and conditions.

\section{PORTION RIGHTS STATEMENT: DISCLAIMER}

If you seek to reuse a portion from an ACS publication, it is your responsibility to examine each portion as published to determine whether a credit to, or copyright notice of, a third party owner was published adjacent to the item. You may only obtain permission via Rightslink to use material owned by ACS. Permission to use any material published in an ACS publication, journal, or article which is reprinted with permission of a third party must be obtained from the third party owner. ACS disclaims any responsibility for any use you make of items owned by third parties without their permission.

\section{REVOCATION}

The American Chemical Society reserves the right to revoke a license for any reason, including but not limited to advertising and promotional uses of ACS content, third party usage, and incorrect figure source attribution.

\section{LICENSE CONTINGENT ON PAYMENT}

While you may exercise the rights licensed immediately upon issuance of the license at the end of the licensing process for the transaction, provided that you have disclosed complete and accurate details of your proposed use, no license is finally effective unless and until full payment is received from you (by CCC) as provided in CCC's Billing and Payment terms and conditions. If full payment is not received on a timely basis, then any license preliminarily granted shall be deemed automatically revoked and shall be void as if never granted. Further, in the event that you breach any of these terms and conditions or any of CCC's Billing and Payment terms and conditions, the license is automatically revoked and shall be void as if never granted. Use of materials as described in a revoked license, as well as any use of the materials beyond the scope of an unrevoked license, may constitute copyright infringement and publisher reserves the right to take any and all action to protect 
its copyright in the materials.

\section{COPYRIGHT NOTICE: DISCLAIMER}

You must include the following copyright and permission notice in connection with any reproduction of the licensed material: "Reprinted ("Adapted" or "in part") with permission from REFERENCE CITATION. Copyright YEAR American Chemical Society."

WARRANTIES: NONE

Publisher makes no representations or warranties with respect to the licensed material.

\section{INDEMNITY}

You hereby indemnify and agree to hold harmless publisher and $\mathrm{CCC}$, and their respective officers, directors, employees and agents, from and against any and all claims arising out of your use of the licensed material other than as specifically authorized pursuant to this license.

\section{NO TRANSFER OF LICENSE}

This license is personal to you or your publisher and may not be sublicensed, assigned, or transferred by you to any other person without publisher's written permission.

\section{NO AMENDMENT EXCEPT IN WRITING}

This license may not be amended except in a writing signed by both parties (or, in the case of publisher, by CCC on publisher's behalf).

\section{OBJECTION TO CONTRARY TERMS}

Publisher hereby objects to any terms contained in any purchase order, acknowledgment, check endorsement or other writing prepared by you, which terms are inconsistent with these terms and conditions or CCC's Billing and Payment terms and conditions. These terms and conditions, together with CCC's Billing and Payment terms and conditions (which are incorporated herein), comprise the entire agreement between you and publisher (and CCC) concerning this licensing transaction. In the event of any conflict between your obligations established by these terms and conditions and those established by CCC's Billing and Payment terms and conditions, these terms and conditions shall control.

\section{JURISDICTION}

This license transaction shall be governed by and construed in accordance with the laws of the District of Columbia. You hereby agree to submit to the jurisdiction of the courts located in the District of Columbia for purposes of resolving any disputes that may arise in connection with this licensing transaction.

\section{THESES/DISSERTATION TERMS}

Publishing implications of electronic publication of theses and dissertation material Students and their mentors should be aware that posting of theses and dissertation material on the Web prior to submission of material from that thesis or dissertation to an ACS journal may affect publication in that journal. Whether Web posting is considered prior publication may be evaluated on a case-by-case basis by the journal's editor. If an ACS journal editor considers Web posting to be "prior publication", the paper will not be accepted for publication in that journal. If you intend to submit your unpublished paper to ACS for publication, check with the appropriate editor prior to posting your manuscript electronically.

If your paper has already been published by ACS and you want to include the text or portions of the text in your thesis/dissertation in print or microfilm formats, please print the 
ACS copyright credit line on the first page of your article: "Reproduced (or 'Reproduced in part') with permission from [FULL REFERENCE CITATION.] Copyright [YEAR]

American Chemical Society." Include appropriate information.

Submission to a Dissertation Distributor: If you plan to submit your thesis to UMI or to another dissertation distributor, you should not include the unpublished ACS paper in your thesis if the thesis will be disseminated electronically, until ACS has published your paper. After publication of the paper by ACS, you may release the entire thesis (not the individual ACS article by itself) for electronic dissemination through the distributor; ACS's copyright credit line should be printed on the first page of the ACS paper.

Use on an Intranet: The inclusion of your ACS unpublished or published manuscript is permitted in your thesis in print and microfilm formats. If ACS has published your paper you may include the manuscript in your thesis on an intranet that is not publicly available. Your ACS article cannot be posted electronically on a publicly available medium (i.e. one that is not password protected), such as but not limited to, electronic archives, Internet, library server, etc. The only material from your paper that can be posted on a public electronic medium is the article abstract, figures, and tables, and you may link to the article's DOI or post the article's author-directed URL link provided by ACS. This paragraph does not pertain to the dissertation distributor paragraph above.

Other conditions:

v1.1

Gratis licenses (referencing $\$ 0$ in the Total field) are free. Please retain this printable license for your reference. No payment is required.

If you would like to pay for this license now, please remit this license along with your payment made payable to "COPYRIGHT CLEARANCE CENTER" otherwise you will be invoiced within $\mathbf{4 8}$ hours of the license date. Payment should be in the form of a check or money order referencing your account number and this invoice number

RLNK10869706.

Once you receive your invoice for this order, you may pay your invoice by credit card. Please follow instructions provided at that time.

Make Payment To:

Copyright Clearance Center

Dept 001

P.O. Box 843006

Boston, MA 02284-3006

If you find copyrighted material related to this license will not be used and wish to cancel, please contact us referencing this license number 2533370713293 and noting the reason for cancellation.

Questions? customercare@copyright.com or +1-877-622-5543 (toll free in the US) or +1-978-646-2777. 


\section{C.6. Permission from American Chemical Society for figure 4.2 in Chapter 4, Reference [7], and figure 5.3 in Chapter 5, Reference [30]}

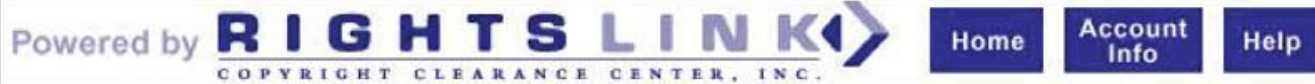

\section{ACS Publications Title: \\ High quality High impuct.}

Enzymes as Working or Inspirational Electrocatalysts for Fuel Cells and Electrolysis

Author: James A. Cracknell et al.

Publication: Chemical Reviews

Publisher: American Chemical Society

Date: Jul 1, 2008

Copyright (c) 2008, American Chemical Society

\section{Logged in as:}

Archana Pandey

Account \#: 3000187610

\section{Order Completed}

Thank you very much for your order.

This is a License Agreement between Archana Pandey ("You") and American Chemical Society ("American Chemical Society"). The license consists of your order details, the terms and conditions provided by American Chemical Society, and the payment terms and conditions.

Get the printable license.

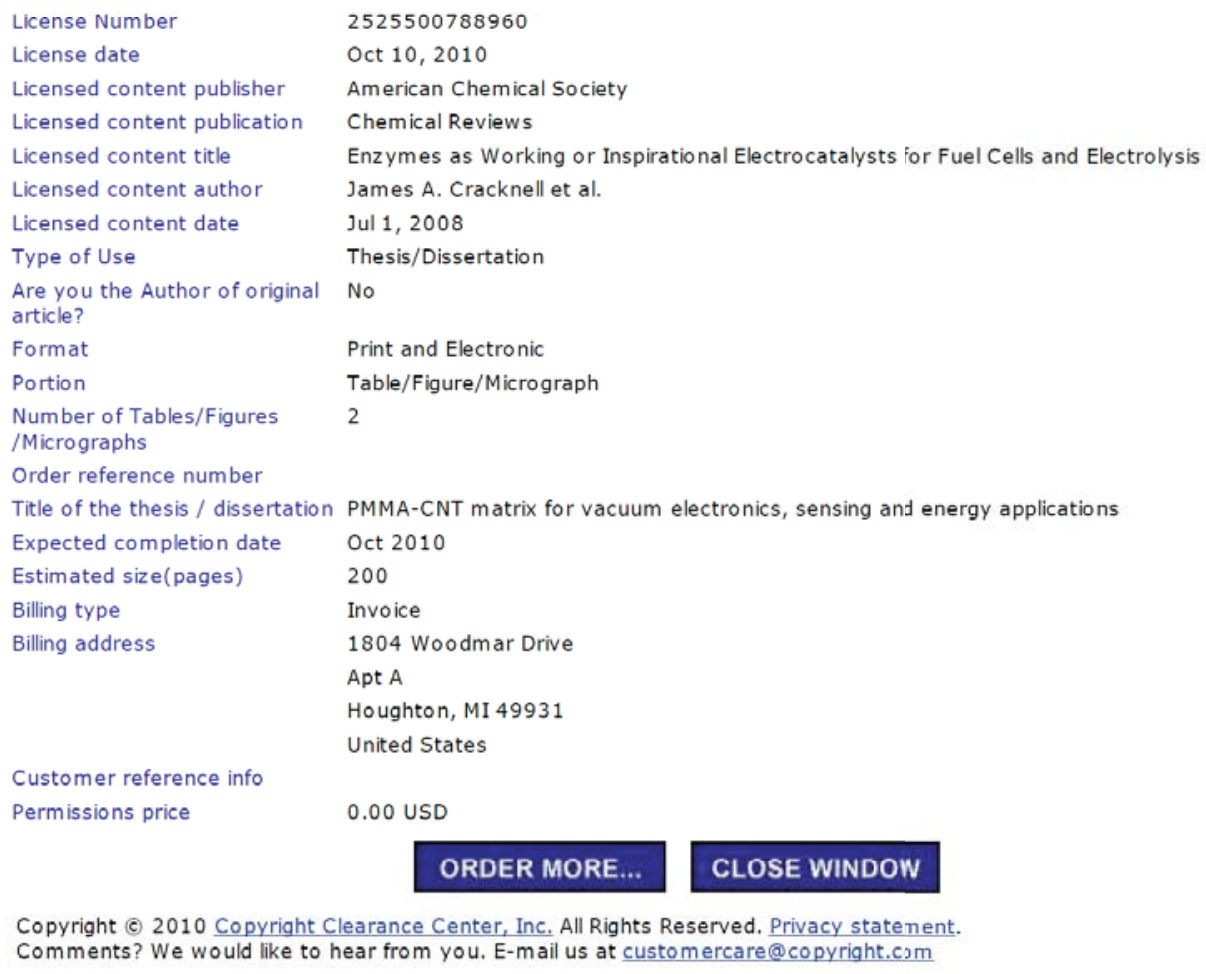




\section{AMERICAN CHEMICAL SOCIETY LICENSE} TERMS AND CONDITIONS

This is a License Agreement between Archana Pandey ("You") and American Chemical Society ("American Chemical Society") provided by Copyright Clearance Center ("CCC"). The license consists of your order details, the terms and conditions provided by American Chemical Society, and the payment terms and conditions.

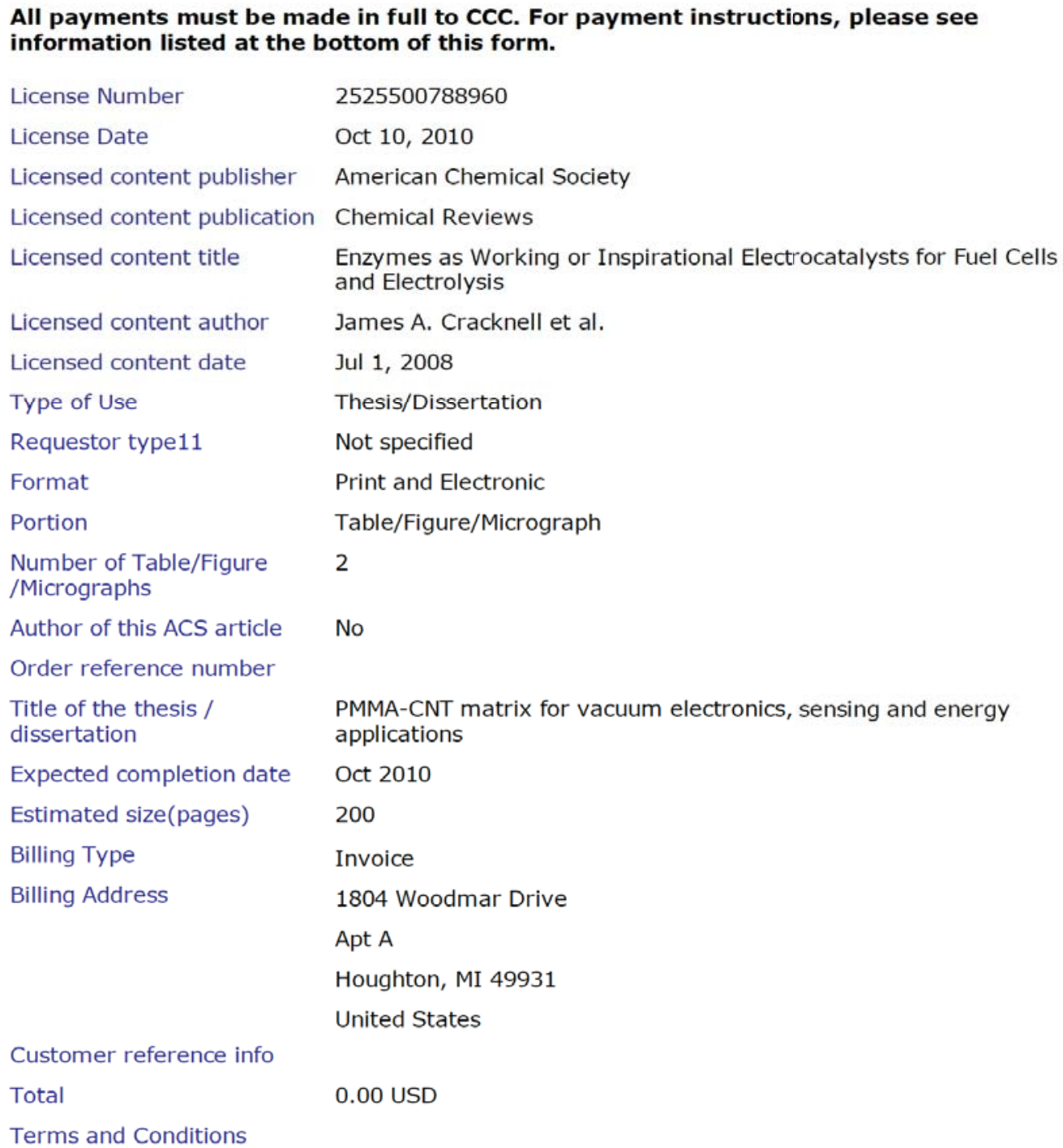

Terms and Conditions

\section{Thesis/Dissertation}




\section{ACS / RIGHTSLINK TERMS \& CONDITIONS \\ THESIS/DISSERTATION}

\section{INTRODUCTION}

The publisher for this copyrighted material is the American Chemical Society. By clicking "accept" in connection with completing this licensing transaction, you agree that the following terms and conditions apply to this transaction (along with the Billing and Payment terms and conditions established by Copyright Clearance Center, Inc. ("CCC"), at the time that you opened your Rightslink account and that are available at any time at

$<$ http://myaccount.copyright.com>).

\section{LIMITED LICENSE}

Publisher hereby grants to you a non-exclusive license to use this material. Licenses are for one-time use only with a maximum distribution equal to the number that you identified in the licensing process; any form of republication must be completed within 60 days from the date hereof (although copies prepared before then may be distributed thereafter).

\section{GEOGRAPHIC RIGHTS: SCOPE}

Licenses may be exercised anywhere in the world.

\section{RESERVATION OF RIGHTS}

Publisher reserves all rights not specifically granted in the combination of (i) the license details provided by you and accepted in the course of this licensing transaction, (ii) these terms and conditions and (iii) CCC's Billing and Payment terms and conditions.

PORTION RIGHTS STATEMENT: DISCLAIMER If you seek to reuse a portion from an ACS publication, it is your responsibility to examine each portion as published to determine whether a credit to, or copyright notice of, a third party owner was published adjacent to the item. You may only obtain permission via Rightslink to use material owned by ACS. Permission to use any material published in an ACS publication, journal, or article which is reprinted with permission of a third party must be obtained from the third party owner. ACS disclaims any responsibility for any use you make of items owned by third parties without their permission.

\section{REVOCATION}

The American Chemical Society reserves the right to revoke a license for any reason, including but not limited to advertising and promotional uses of ACS content, third party usage, and incorrect figure source attribution.

\section{LICENSE CONTINGENT ON PAYMENT}

While you may exercise the rights licensed immediately upon issuance of the license at the end of the licensing process for the transaction, provided that you have disclosed complete and accurate details of your proposed use, no license is finally effective unless and until full payment is received from you (by CCC) as provided in CCC's Billing and Payment terms and conditions. If full payment is not received on a timely basis, then any license preliminarily granted shall be deemed automatically revoked and shall be void as if never granted. Further, in the event that you breach any of these terms and conditions or any of CCC's Billing and Payment terms and conditions, the license is automatically revoked and shall be void as if never granted. Use of materials as described in a revoked license, as well as any use of the materials beyond the scope of an unrevoked license, may constitute copyright infringement and publisher reserves the right to take any and all action to protect 
its copyright in the materials.

\section{COPYRIGHT NOTICE: DISCLAIMER}

You must include the following copyright and permission notice in connection with any reproduction of the licensed material: "Reprinted ("Adapted" or "in part") with permission from REFERENCE CITATION. Copyright YEAR American Chemical Society."

\section{WARRANTIES: NONE}

Publisher makes no representations or warranties with respect to the licensed material.

\section{INDEMNITY}

You hereby indemnify and agree to hold harmless publisher and CCC, and their respective officers, directors, employees and agents, from and against any and all claims arising out of your use of the licensed material other than as specifically authorized pursuant to this license.

\section{NO TRANSFER OF LICENSE}

This license is personal to you or your publisher and may not be sublicensed, assigned, or transferred by you to any other person without publisher's written permission.

\section{NO AMENDMENT EXCEPT IN WRITING}

This license may not be amended except in a writing signed by both parties (or, in the case of publisher, by CCC on publisher's behalf).

\section{OBJECTION TO CONTRARY TERMS}

Publisher hereby objects to any terms contained in any purchase order, acknowledgment, check endorsement or other writing prepared by you, which terms are inconsistent with these terms and conditions or CCC's Billing and Payment terms and conditions. These terms and conditions, together with CCC's Billing and Payment terms and conditions (which are incorporated herein), comprise the entire agreement between you and publisher (and CCC) concerning this licensing transaction. In the event of any conflict between your obligations established by these terms and conditions and those established by CCC's Billing and Payment terms and conditions, these terms and conditions shall control.

\section{JURISDICTION}

This license transaction shall be governed by and construed in accordance with the laws of the District of Columbia. You hereby agree to submit to the jurisdiction of the courts located in the District of Columbia for purposes of resolving any disputes that may arise in connection with this licensing transaction.

\section{THESES/DISSERTATION TERMS}

Publishing implications of electronic publication of theses and dissertation material Students and their mentors should be aware that posting of theses and dissertation material on the Web prior to submission of material from that thesis or dissertation to an ACS journal may affect publication in that journal. Whether Web posting is considered prior publication may be evaluated on a case-by-case basis by the journal's editor. If an ACS journal editor considers Web posting to be "prior publication", the paper will not be accepted for publication in that journal. If you intend to submit your unpublished paper to ACS for publication, check with the appropriate editor prior to posting your manuscript electronically.

If your paper has already been published by ACS and you want to include the text or portions of the text in your thesis/dissertation in print or microfilm formats, please print the 
ACS copyright credit line on the first page of your article: "Reproduced (or 'Reproduced in part') with permission from [FULL REFERENCE CITATION.] Copyright [YEAR]

American Chemical Society." Include appropriate information.

Submission to a Dissertation Distributor: If you plan to submit your thesis to UMI or to another dissertation distributor, you should not include the unpublished ACS paper in your thesis if the thesis will be disseminated electronically, until ACS has published your paper. After publication of the paper by ACS, you may release the entire thesis (not the individual ACS article by itself) for electronic dissemination through the distributor; ACS's copyright credit line should be printed on the first page of the ACS paper.

Use on an Intranet: The inclusion of your ACS unpublished or published manuscript is permitted in your thesis in print and microfilm formats. If ACS has published your paper you may include the manuscript in your thesis on an intranet that is not publicly available. Your ACS article cannot be posted electronically on a publicly available medium (i.e. one that is not password protected), such as but not limited to, electronic archives, Internet, library server, etc. The only material from your paper that can be posted on a public electronic medium is the article abstract, figures, and tables, and you may link to the article's DOI or post the article's author-directed URL link provided by ACS. This paragraph does not pertain to the dissertation distributor paragraph above.

Other conditions:

$\mathrm{vl} .1$

Gratis licenses (referencing $\$ 0$ in the Total field) are free. Please retain this printable license for your reference. No payment is required.

If you would like to pay for this license now, please remit this license along with your payment made payable to "COPYRIGHT CLEARANCE CENTER" otherwise you will be invoiced within $\mathbf{4 8}$ hours of the license date. Payment should be in the form of a check or money order referencing your account number and this invoice number RLNK10863511.

Once you receive your invoice for this order, you may pay your invoice by credit card. Please follow instructions provided at that time.

Make Payment To:

Copyright Clearance Center

Dept 001

P.O. Box 843006

Boston, MA 02284-3006

If you find copyrighted material related to this license will not be used and wish to cancel, please contact us referencing this license number 2525500788960 and noting the reason for cancellation.

Questions? customercare@copyright.com or +1-877-622-5543 (toll free in the US) or +1-978-646-2777. 